\title{
Investigation and Development of a Real-Time On-Site Condition Monitoring System for Induction Motors
}

\author{
by
}

\section{Syaiful Bakhri}

Thesis submitted for the degree of

\section{Master of Engineering Science}

School of Electrical and Electronic Engineering

Faculty of Engineering, Computer and Mathematical Science

The University of Adelaide

Australia

August 2008

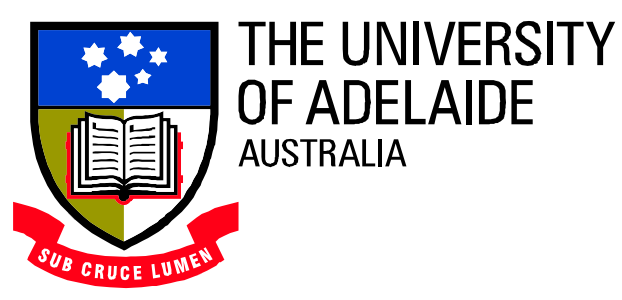




\section{Contents}

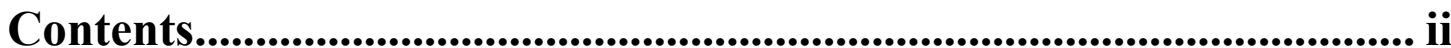

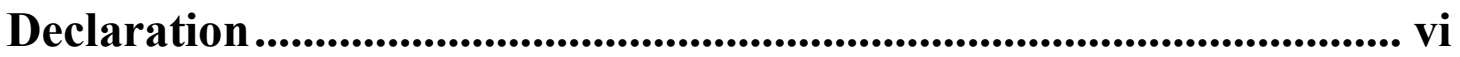

List of Figures ........................................................................................ vii

List of Abbreviations and Symbols........................................................ xiv

List of Abbreviations ………………………………………….......................... xiv

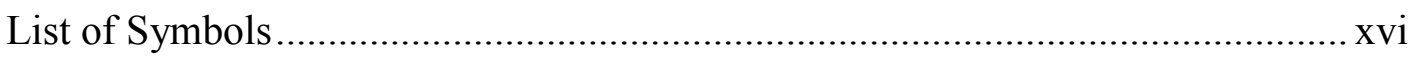

Chapter 1

Overview of Condition Monitoring of Induction Motor .........................1

1.1 Overview and Problem .......................................................................................... 1

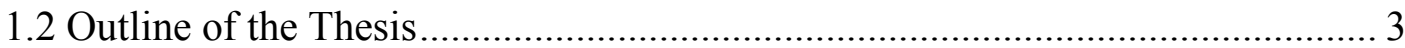

\section{Chapter 2}

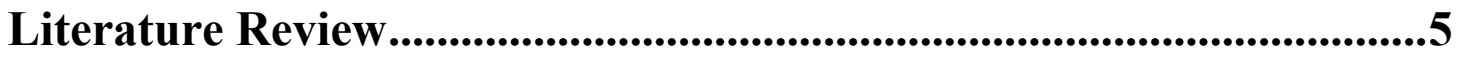

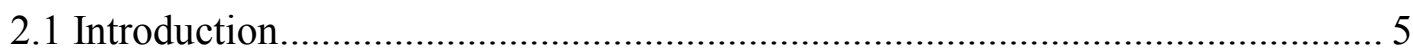

2.2 Approaches used in Condition Monitoring Systems ………………………..... 5

2.3 Common Faults of Induction Motor ................................................................... 7

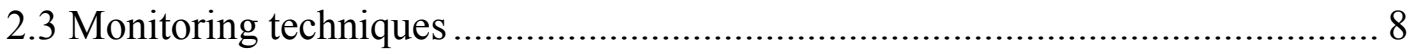

2.2.1 Vibration Monitoring ........................................................................... 16

2.2.2 Stator Current Monitoring....................................................................... 18

2.2.3 Stator Voltage Monitoring ....................................................................... 20

2.2.4 Flux Leakage Monitoring ………………………................................. 20

2.3 Data Analysis and Diagnostics .................................................................... 22

2.4 Condition Monitoring Devices .................................................................... 24

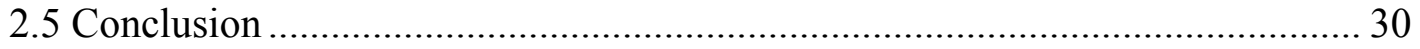

\section{Chapter 3}

Induction Motors and Fault Frequencies ...........................................32

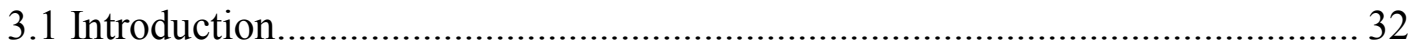

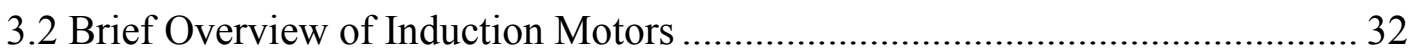


3.2.1 Overview of Induction Motor Construction 33

3.2.2 Overview of the Principle of Operation...................................................... 35

3.2.3 Overview of Synchronous Speed and Slip............................................ 36

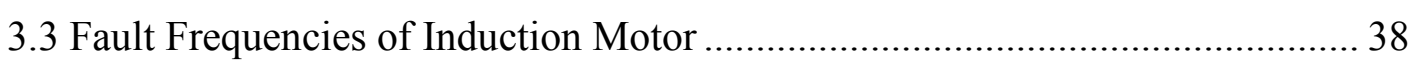

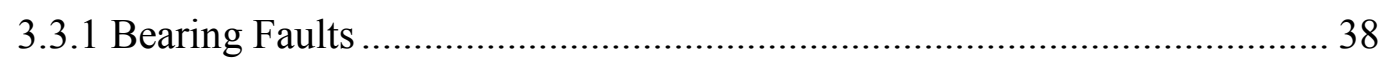

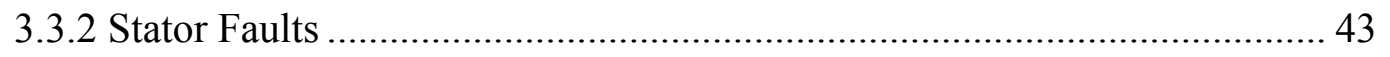

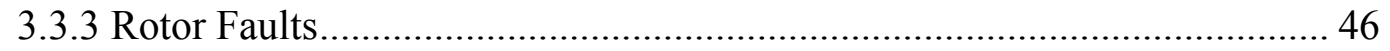

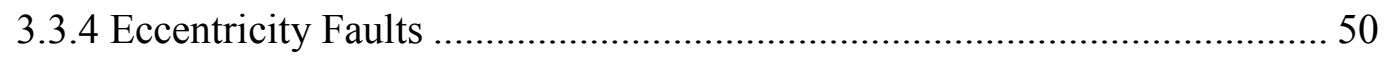

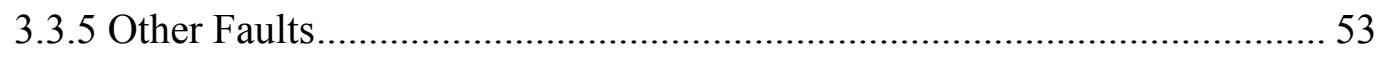

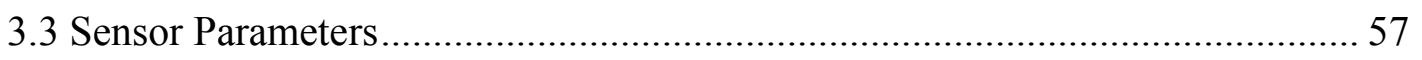

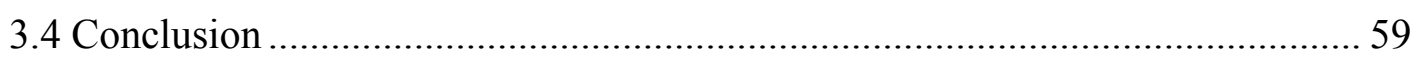

\section{Chapter 4}

\section{Development of a CompactRIO-Based Condition Monitoring System}

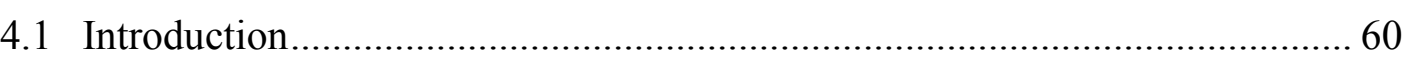

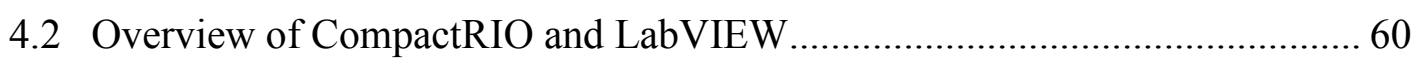

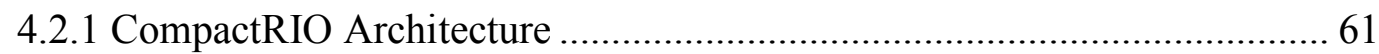

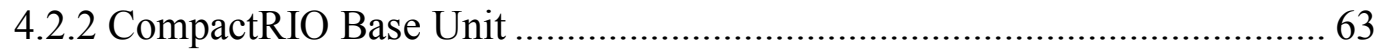

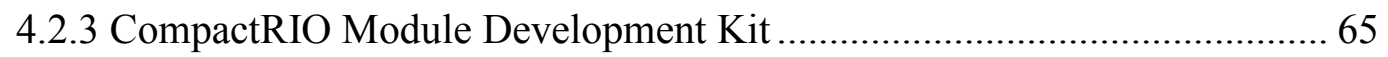

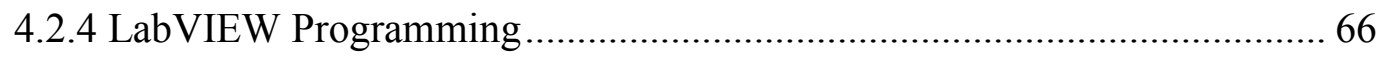

4.2.5 CompactRIO Condition Monitoring Segmented Development.................. 68

4.3 The CompactRIO Indicator Module ............................................................. 70

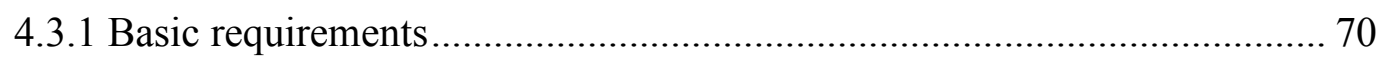

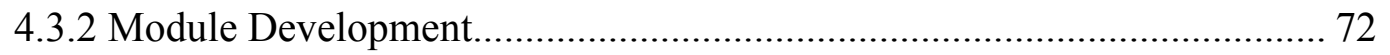

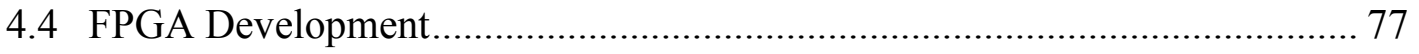

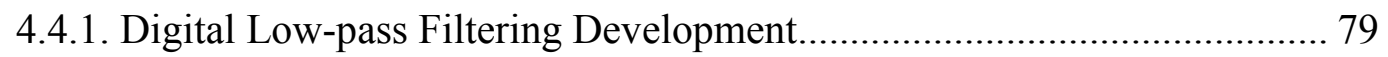

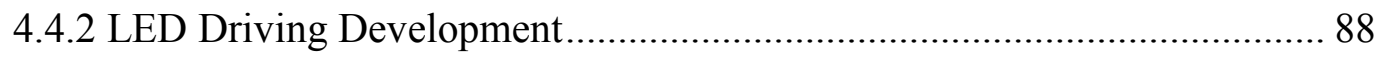

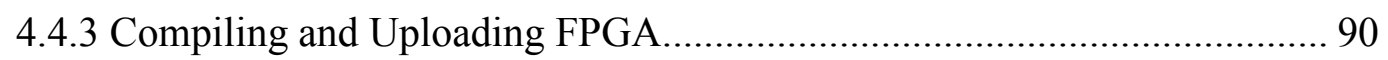

4.5 Real-time Condition Monitoring Software Development ............................. 91

4.5.1. Host to FPGA Synchronization and Data Transfer.................................. 93

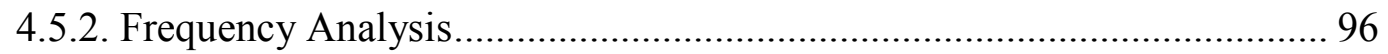

4.5.3. Fault Frequency Analysis ................................................................... 100 
4.5.3.1 Fundamental Frequencies and Slip Calculation.................................. 103

4.5.3.2 Fault Frequency Predictors and The Sub VI........................................ 105

4.5.3.2.1 Eccentricity Fault ........................................................................... 105

4.5.3.2.2 Broken Rotor Bar Fault................................................................... 106

4.5.3.2.3 Stator Shorted Turn Fault................................................................... 109

4.5.3.2.4 Set-up Related Fault....................................................................... 110

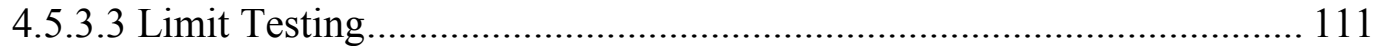

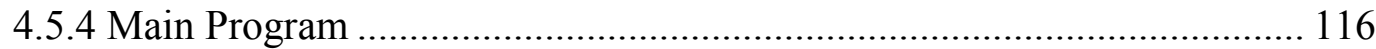

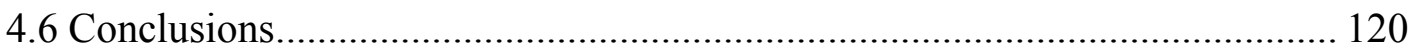

\section{Chapter 5}

\section{Experimental Evaluation of the CompactRIO-Based Condition}

\section{Monitoring System ..................................................................................121}

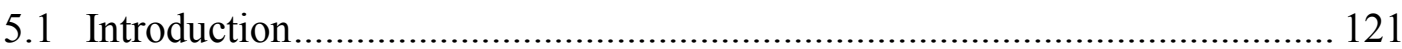

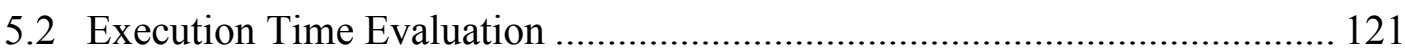

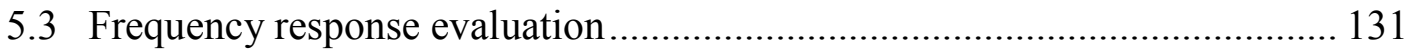

5.4 Induction Motor Fault Evaluation.................................................................. 136

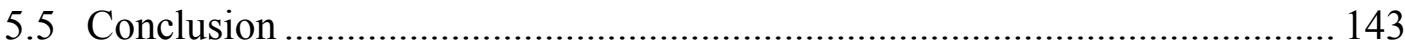

\section{Chapter 6}

General Conclusions and Suggestions................................................144

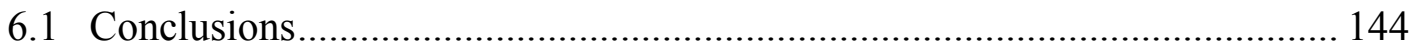

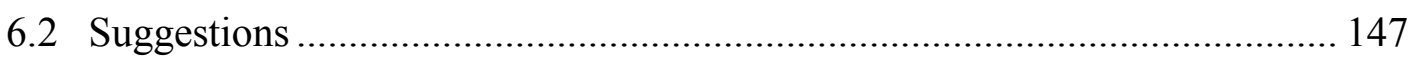

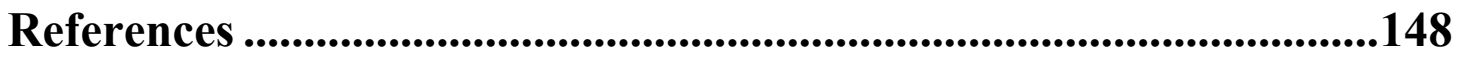

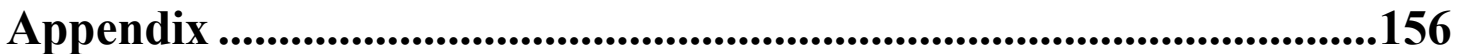

A.1 The Details PCB of CompactRIO indicator module ................................... 156 


\section{Abstract}

This thesis presents an investigation and development of a real-time on-site condition monitoring system for induction motors. Induction motors are employed in various industries as an essential machine. In order to prevent catastrophic faults during its serviceable life, condition monitoring of induction motors is commonly used in industrial applications to maintain safety and reliability of plant operation. The current practice in condition monitoring primarily involves using various forms of mobile or portable devices, usually with a single sensor input to perform tests at regular intervals. However, such devices and monitoring services can be expensive and require an experienced operator for reliable decisions. Therefore, this thesis investigates an alternative low-cost solution for continuous condition monitoring of induction machines using multiple sensors, which can be located next to a machine under test and can provide condition information using indicator lights for quick diagnosis.

The thesis provides hardware and software implementation details using an FPGA based CompactRIO platform. The CompactRIO embedded reconfigurable platform incorporates an FPGA, an analog input module, a real-time host controller and a custom-made indicator module as an on-site monitoring system. The CompactRIO custom-made indicator module utilizes bi-colour LEDs and requirements of CompactRIO MDK to display each level fault. Furthermore, a data acquisition and monitoring system was developed under LabVIEW FPGA environment and LabVIEW Real Time software. The system that has been successfully designed has had its performances and capabilities evaluated through several tests. In addition, real faults are also introduced to demonstrate the system's performance. The results show that the CompactRIO system is capable of being implemented as condition monitoring system, especially as an early warning unit. The early warning information obtained from this system can be used as valuable data for further detailed fault assessment. 


\section{Declaration}

This work contains no material which has been accepted for the award of any other degree or diploma in any university or other tertiary institution and, to the best of my knowledge and belief, contains no material previously published or written by any other person, except where due reference has been made in the text. I give consent to this copy of my thesis, when deposited in the University Library, being made available for loan and photocopying, subject to the provisions of the Copyright Act 1968.

Signed :

Date : 


\section{Acknowledgement}

Firstly, I wish to express sincere gratitude to my principal supervisor Associate Professor Dr. Nesimi Ertugrul for his guidance, inspiration, motivation, support and knowledge during my research. He has encouraged improvement and development. He has also given essential support by providing a CompactRIO base unit, LabVIEW software and CompactRIO MDK.

I would like to thank to Dr. Wen L. Soong and Dr. Said Al-Sarawi for the inspiration, guidance, and suggestion that have enriched this project.

I would like to thank all the technical staff for their help and assistance during my research in power laboratory. I would also like to thank the information technology support staff, Mr Mark Innes and Mr Anthony Schueller, and school store staff $\mathrm{Mr}$ Danny Di Giacomo, for their help. I would like especially to acknowledge Mr. Pavel Simcik for the PCB development.

I would like to thank and acknowledge Ms Ann Nobel for the editorial assistance during my thesis writing.

An AusAID Australian Development Scholarship provided me with support during my studies, for which I am grateful.

I am grateful to every member of the School of Electrical and Electronic Engineering, and staff of University of Adelaide International student centre, especially Ms Niranjala Seimon, who has helped me during my stay here.

Thanks to my family, my beloved wife, and my children, for their support, patience, encouragement, and understanding during my studies. Thanks also to my relatives in Indonesia for supporting me throughout my candidature.

Syaiful Bakhri

August 2008 


\section{List of Figures}

Figure 1.1 Cut-away view of an induction motor ...................................................... 1

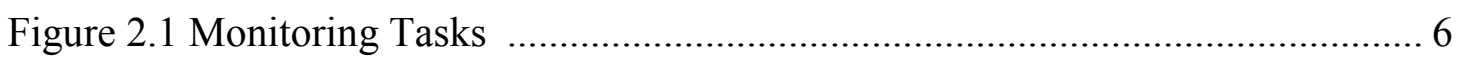

Figure 2.2 Distribution of common faults of electric motors according to .................. 8

Figure 2.3 Condition monitoring of induction motors ............................................... 9

Figure 2.4 (a) Accelerometer, (b) velocity, and (c) displacement transducers for vibration monitoring 17

Figure 2.5 An example of (a) Hall-effect sensor, (b) CT sensor, and (c) Rogowski coil for stator current monitoring ........................................................... 18

Figure 2.6 A differential voltage measurement device for voltage monitoring........... 20

Figure 2.7 Flux region which can be used to detect fault in induction motors............ 21

Figure 2.8 A commercial external flux coil for flux leakage monitoring................... 21

Figure 2.9 A complete structure of a condition monitoring device ............................ 24

Figure 2.10 Commercial handheld (MicroVibe-P), portable (DLI Watchman and

OR35/36), and permanent on-line (VibroControl 4000 and VXI SO analyzer) condition monitoring devices ............................................. 25

Figure 2.11 Example of a data processing board for condition monitoring (a) ISA

DAS-8 Metrabyte Inc data acquisition card, (b) DSP TMS320C32 board,

(c) Altera NIOS II soft core and a Stratix FPGA board.... 27

Figure 2.12 A CompactRIO base unit ..................................................................... 29

Figure 3.1 Cutaway of squirrel cage induction motor showing main parts ................. 33

Figure 3.2 Stator construction: (a) laminations, (b) coil insertions, and (c) a complete

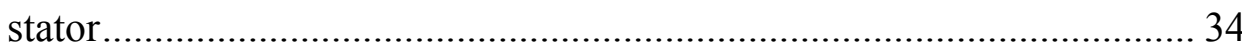

Figure 3.3 Cross-section of (a) a squirrel cage rotor, and (b) a complete arrangement

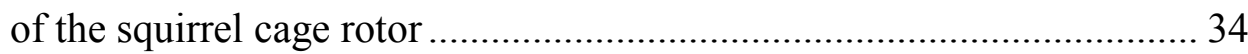

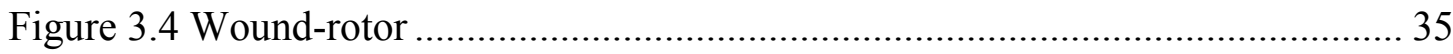

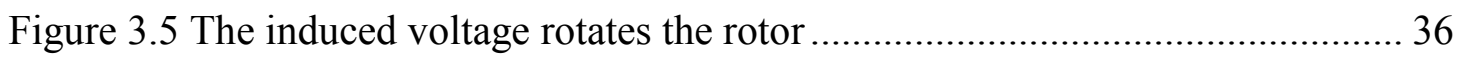

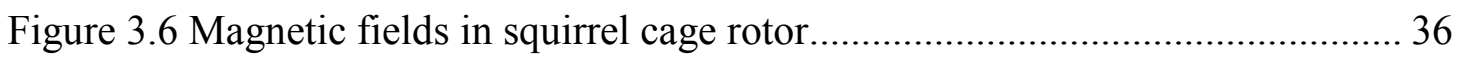

Figure 3.7 (a) Stator winding arrangement and (b) instantaneous value of currents and magnetic flux distribution produced ................................................. 37

Figure 3.8 Cut-away of ball and roller bearing type shows main parts of bearing...... 39

Figure 3.9 Cause of failure in bearing of induction motor. ....................................... 40 
Figure 3.10 Bearing dimension and frequencies of bearing-related faults 41

Figure 3.11 Stator-related fault frequencies of vibration monitoring. 46

Figure 3.12 Broken rotor bar, bad joint and shorted lamination fault frequencies of vibration monitoring 49

Figure 3.13 Loose rotor bar fault frequencies of vibration monitoring. ..................... 50

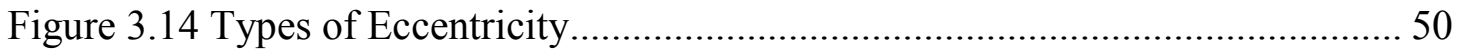

Figure 3.15 Eccentricity fault frequencies of vibration monitoring .......................... 52

Figure 3.16 Types of mechanical unbalance …....................................................... 53

Figure 3.17 Fault frequency of mechanical unbalance........................................... 54

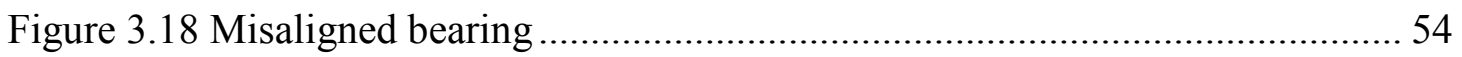

Figure 3.19 Misalignment fault frequencies using vibration monitoring .................... 55

Figure 3.20 Three types of fault frequencies of looseness using vibration monitoring56

Figure 3.21 Supply phasing problem fault frequencies .57

Figure 3.22 Potential position of sensors to gain comprehensive result of condition monitoring when a motor is coupled with a load .................................. 58

Figure 4.1 Four types of CompactRIO configuration: (a) embedded modular, (b) embedded integrated, (c) R-series expansion, and (d) remote high-speed

Figure 4.2 CompactRIO embedded architecture 62

Figure 4.3 CompactRIO Module Development Kit shows empty custom module shells, and development software 65

Figure 4.4 LabVIEW programming components 67

Figure 4.5 Segmented development of CompactRIO embedded condition monitoring 68

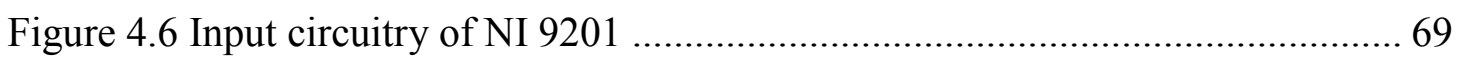

Figure 4.7 CompactRIO communication mode ....................................................... 71

Figure 4.8 CompactRIO basic block diagram ...................................................... 73

Figure 4.9 Schematic diagram of the CompactRIO indicator module ....................... 73

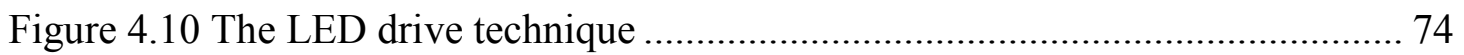

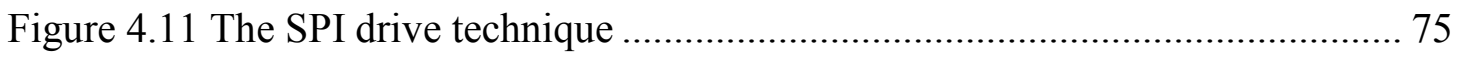

Figure 4.12 (a). CompactRIO indicator circuit, (b) CompactRIO indicator module, and

(c) CompactRIO indicator module attached on CompactRIO base unit.. 76

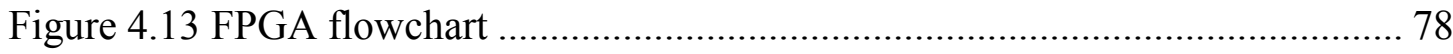


Figure 4.14. (a) Simulation results of the low pass filter design and (b) the zoom frequency response around the edge of the pass band showing the cut-off frequency. 80

Figure 4.15 (a) Calculated pole-zero plots and (b) calculated step response of the low pass filter.

Figure 4.16 (a) Frequency spectra of simulation input random signals and (b) frequency spectra after low pass floating-point Butterworth filter..... 82

Figure 4.17 Comparison of floating-point and fixed-point frequency response. 84

Figure 4.18 A fixed-point model of low pass filter which has quantizers 84

Figure 4.19 Comparison of floating-point and fixed-point simulation result of 86

Figure 4.20 Comparison of floating-point and fixed-point simulation result of random input signal 86

Figure 4.21 Low Pass Butterworth Filter. 87

Figure 4.22 Timing diagram of NLSF 595 LED driver.... 88

Figure 4.23 FPGA indicator module. 89

Figure 4.24 Block diagram VI of the indicator module 90

Figure 4.25 (a) Compiling process of an FPGA VI, and (b) uploading a bit file to the FPGA target

Figure 4.26 Flowchart of condition monitoring software in the real-time host controller 92

Figure 4.27 Basic data transfer and synchronization 94

Figure 4.28 Data synchronization between CompactRIO FPGA and main program CompactRIO Real-Time Host 95

Figure 4.29 Sub VI of frequency analysis : (a) A sub VI of scaling voltage to Specific Engineering Unit (SVL Scale Voltage to EU) and (b) A sub VI of computing FFT spectrum (SVFA FFT Spectrum (Mag-Phase)) 97

Figure 4.30 A sub VI of harmonic analysis (SVT THD and Harmonics Components) 103

Figure 4.31 Sub VI of slip frequency calculation (a) icon and connector pane and (b) block diagram

Figure 4.32 Waveform graphs of (a) fundamental line frequency captured by voltage monitoring, and (b) fundamental rotor frequency captured by vibration monitoring. 104 
Figure 4.33 Sub VI of mix eccentricity calculation: (a) icon and connector pane and

(b) block diagram. 106

Figure 4.34 Sub VI of broken rotor bar calculation using Equation 3.16: (a) icon and connector pane and (b) block diagram..... 107

Figure 4.35 Sub VI of broken rotor bar calculation using Equation 3.17: (a) icon and connector pane and (b) block diagram 108

Figure 4.36 Sub VI of interturn fault calculation: (a) icon and connector pane, and (b) block diagram. 109

Figure 4.37 Icon of set-up related faults Sub VI................................................. 110

Figure 4.38 Block diagram of set-up related faults Sub VI................................... 111

Figure 4.39 Sub VI of peak searching ............................................................. 112

Figure 4.40 Peak search technique of the sub VI in Figure 4.38 ............................ 112

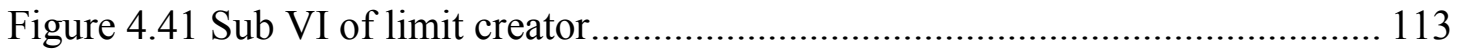

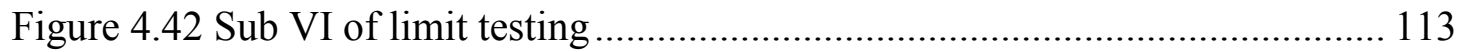

Figure 4.43 Sub VI of three limit thresholds of severity level ................................ 114

Figure 4.44 Example of limit testing result ........................................................... 115

Figure 4.45 Sub VI of conversion from severity level to LED colours .................... 116

Figure 4.46 First part of main program of real-time condition monitoring ............... 117

Figure 4.47 Second part of main program of real-time condition monitoring .......... 118

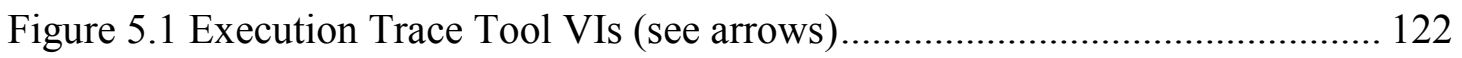

Figure 5.2 Execution Trace Tool front panel...................................................... 123

Figure 5.3 Zoom view of thread event of Execution Trace Tool demonstrates length of execution time 124

Figure 5.4 Zoom view of VIs event of Execution Trace Tool shows execution time of main and sub VIs ..................................................................... 125

Figure 5.5 Zoom view of VIs event of Execution Trace Tool shows execution time of algorithms of frequency analysis 126

Figure 5.6 Real-time system manager shows memory usage and CPU usage during a measurement 130

Figure 5.7 Frequency response of monitoring system without low pass filter and using low pass filter. 132

Figure 5.8 Zoom view of specific peak with : (a) 512 lines (b) 1024 lines (c) 2048 lines and (d) 4096 lines 133

Figure 5.9 Examples of various windowing techniques can be performed 134 
Figure 5.10 User interface of the CompactRIO based condition monitoring system 136

Figure 5.11 A zoomed section of the frequency spectrum indicating the sidebands in circles, under a broken rotor bar fault

Figure 5.12 A zoomed section of the stator current frequency spectrum indicating the sidebands in circles, under an eccentricity

Figure 5.13 A zoomed section of the frequency spectrum of flux leakage and vibration under a shorted turn 138

Figure 5.14 Real-time current/voltage waveforms and their frequency spectra under eccentricity fault.

Figure 5.15 Zoom of real-time three axis vibration and axial flux leakage signals with corresponding zoom of frequency spectra under a multiple fault. 140

Figure A.1 PCB layout of CompactRIO MDK LED indicator module (a) top view and (b) bottom view 156 


\section{List of Tables}

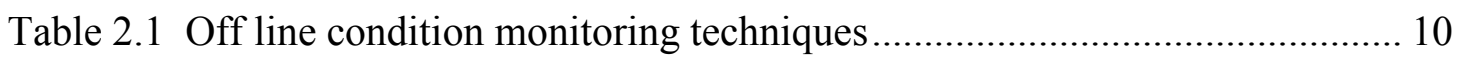

Table 2.2 On-line condition monitoring techniques .............................................. 11

Table 2.3 Studies of common faults in induction motors using vibration, stator current, stator voltage and flux leakage monitoring ............................... 15

Table 2.4 Advantages and disadvantages types of condition monitoring devices...... 26

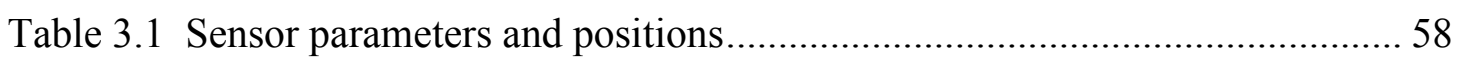

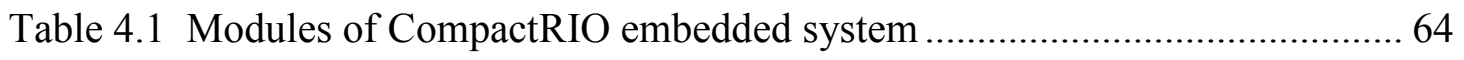

Table 4.2 Key pins of the SPI template in Operation Mode of CompactRIO custom module development ........................................................................ 72

Table 4.3 Low pass floating-point Butterworth filter specification........................... 80

Table 4.4 IIR low pass Butterworth filter fixed-point structure setting.................... 83

Table 4.5 IIR low pass Butterworth filter fixed-point modeling coefficients ............ 85

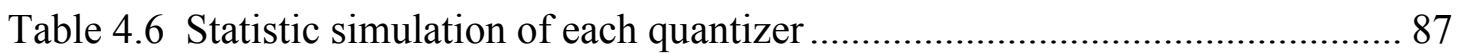

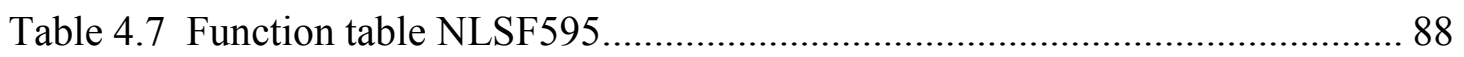

Table 4.8 LabVIEW VI functions used for frequency analysis................................96

Table 4.9 LabVIEW VI functions used for frequency analysis (continued) .............. 97

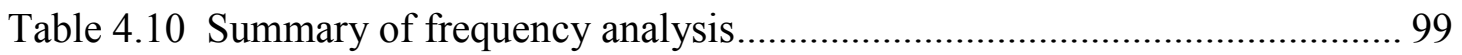

Table 4.11 Custom and built-in VIs implemented for fault frequency analysis....... 100

Table 4.12 Fault frequency predictor of broken rotor bar using Equation 3.16 ...... 107

Table 4.13 Fault frequency predictor of broken rotor bar using Equation 3.17 ...... 109

Table 4.14 Fault frequency predictor of stator interturn fault ............................... 110

Table 4.15 Relation of the limit mask with the severity level area ........................ 115

Table 5.1 Description of several thread events .................................................... 127

Table 5.2 Average execution times of the various sections of the signal processing

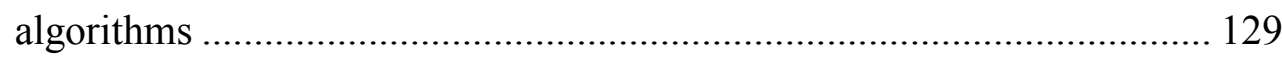

Table 5.3 Average execution times of the CompactRIO fault analysis stage........... 129

Table 5.4 The comparison of the width windows at certain amplitude .................... 134

Table 5.5 Performance of peak detection under twenty consecutive tests .............. 141 


\section{List of Abbreviations and Symbols List of Abbreviations}

$\mathrm{AC}$

ADC

AlBar

$\mathrm{BNC}$

CAN

CompactRIO

$\mathrm{CT}$

$\mathrm{CuDC}$

CuBar

DMA

DRAM

DSP

EEPROM

EPRI

FFT

FIFO

FIR

FTP

FPGA

GND

IEEE-IAS

IIR

$\mathrm{I} / \mathrm{O}$

HTTP

IR

ISA

LAN

LED
Alternating Current

Analog to Digital Converter

Aluminum Bars

Bayonette Neil-Concelman (connector)

Controller Access Network

Compact Reconfigurable Input Output

Clip on Transformer

Copper Die Cast

Copper Bar

Direct Memory Access

Dynamic Random Access Memory

Digital Signal Processor

Electrically Erasable Programmable Read-Only Memory

Electric Power Research Institute

Fast Fourier Transform

First In First Out

Finite Impulse Response

File Transfer Protocol

Field Programmable Gate Array

Ground

Institute of Electrical and Electronic Engineering-Industry

Application Society

Infinite Impulse Response

Input/Output

Hypertext Transfer Protocol

Insulation Resistance

Industry Standard Architecture

Local Area Network

Light Emitting Diode 


\begin{tabular}{|c|c|}
\hline $\mathrm{LCD}$ & Liquid Crystal Display \\
\hline LabVIEW & Laboratory Virtual Instrumentation Engineering Workbench \\
\hline MCSA & Motor Current Stator Analysis \\
\hline MDK & Module Development Kit \\
\hline MISO & Master-In Slave-Out \\
\hline MOSI & Master-Out Slave-Input \\
\hline MSE & Mean Square Error \\
\hline NI & National Instrument \\
\hline $\mathrm{OE}$ & Output Enable \\
\hline PDA & Personal Data Assistant \\
\hline PI & Polarization Index \\
\hline $\mathrm{PC}$ & Personal Computer \\
\hline PCI & Peripheral Component Interconnect \\
\hline PXI & PCI Extension for Instrumentation \\
\hline $\mathrm{RCK}$ & Register Clock \\
\hline SCK & Serial Clock \\
\hline SI & Serial Input \\
\hline $\mathrm{SQH}$ & Serial Output \\
\hline SPI & Serial Peripheral Interface \\
\hline SPI_CLK & SPI Clock \\
\hline SPI_CS & SPI Chip Select \\
\hline $\mathrm{TCP} / \mathrm{IP}$ & Transmission Control Protocol/ Internet Protocol \\
\hline RAM & Random Access Memory \\
\hline RMS & Root Mean Square \\
\hline VI & Virtual Instrument \\
\hline VXI & VME eXtensions for Instrumentation \\
\hline
\end{tabular}




\section{List of Symbols}

$\theta$

$D_{B}$

$D_{P}$

$f$

$F_{B F}$

$F_{B R B}$

$F_{C F}$

$F_{E C C}$

$F_{\text {ORF }}$

$F_{\text {IRF }}$

$F_{L}$

$F_{P}$

$F_{R}$

$F_{R B P F}$

$F_{S F}$

$F_{S L O T+E C C}$

IOL,MAX

$p$

$R$

$\mathrm{R}$

$s$

tol

$N_{B}$

$N_{R}$

$N_{S}$

$\mathrm{V}_{\mathrm{cc}, \mathrm{MAX}}$

$\mathrm{V}_{\mathrm{f}, \mathrm{LED}, \mathrm{MIN}}$

$\mathrm{V}_{\text {OL,MIN }}$
Ball contact angle

Ball diameter

Pitch diameter

Frequency of the source

Ball fault frequency

Detectable broken rotor bar frequency

Cage fault frequency

Eccentricity fault frequency

Outer race fault frequency

Inner race fault frequency

Power supply line frequency

Pole pass frequency

Rotor or shaft frequency

Rotor bar pass frequency

Stator fault frequency

Slot and eccentricity fault frequency

Maximum current to be allowed

Number of pole pairs

Number of rotor slots

Resistor centre value

Slip

Tolerance of resistor

Number of balls

Rotor speed

Synchronous speed of the motor

Maximum power supply voltage

Minimum LED forward voltage at $\mathrm{I}_{\mathrm{OL}, \mathrm{MAX}}$

Minimum output low voltage from the LED driver at IOL,MAX 


\section{Chapter 1}

\section{Overview of Condition Monitoring of Induction Motor}

\subsection{Overview and Problem}

Electric motors were first developed 170 years ago, and are now widely used in industry. The electric motor market is estimated to be growing at a rate between 3 $\%$ to more than $10 \%$ in 2008 [1]. There are about $3 \mathrm{~kW}$ of electric motors are utilized per person in developed countries [1]. In other words, electric motors have become increasingly essential to our industrialized society.

Among the different types of electric motors, the induction motor is the most popular with around $85 \%$ of the electric motors. The primary reasons are that they are simple, inexpensive, highly reliable, and easy to maintain. The induction motor ratings may vary from a few watts to tens of MW, which suits a wide rage of industrial requirements. Figure 1.1 shows an example of a cut-away view of a typical induction motor and indicates the main parts including the stator, rotor, shaft and bearings.

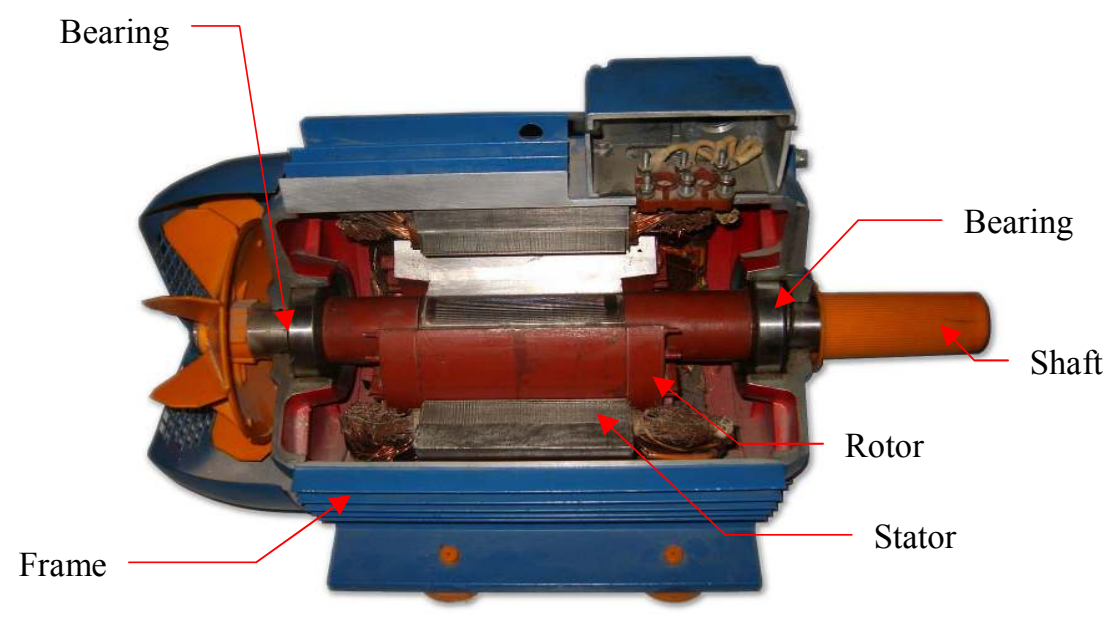

Figure 1.1 Cut-away view of an induction motor [2] 
As is commonly known, since first development, the critical parts of induction motors as shown in Figure 1.1 have been refined steadily to include various designs, features and manufacturing specifications to achieve high reliability, efficiency and performance. However, faults may still occur in these motors. This is because induction motors are also subject to numerous environmental and operational hazards under real operating conditions, which may lead to some undesirable mechanical and electrical stresses during operation [3]. These stresses, in turn, lead to accumulated faults and propagate failure [4]. Furthermore, these faults, especially in critical electro-mechanical parts, may drive to excessive downtimes causing revenue loss, and reduced power output for an industry [3,5]. Moreover, the faults can reduce the safe operation of industrial plants, such as nuclear power plants. Therefore, in order to avoid the negative impacts of various faults, real-time condition monitoring of induction motors is needed.

Based on monitoring results, operators can determine the current condition of an induction motor. Condition monitoring can provide information about potential faults or unidentified failures, which can be used to keep these motors in operation for a longer time without programmed maintenance. In addition, over a period of time, trended results can be used to provide a reliable failure time which can identify failed parts, as well as offer a maintenance time table. These steps, also known as predictive maintenance, involve comprehensive data collection and analysis allowing maintenance to be carried out in a systematic manner before failure of induction motors.

Condition monitoring which is implemented in a predictive maintenance strategy is also a crucial investment factor, as it can be costly due to complex techniques and expensive tools [6]. For example, some commercial advanced devices which adopt an expert system are expensive [7]. These devices provide comprehensive analysis through database management software and on-line real-time distributed data acquisition. However, the cheaper devices such as portable data analyzers usually require an expert, even a certified engineer, to predict the faults of the induction motors [7].

During the past decade, condition monitoring system development has dramatically changed in line with the computer revolution. Some of these condition monitoring devices utilized Digital Signal Processor (DSP), Personal Data Assistant 
(PDA), or computers as signal analyzers [6, 8-12]. Furthermore, a few studies have been performed to developed condition monitoring devices employing PC data acquisition cards, DSP or FPGA. Few of these studies consider semi-compact, lowcost and reconfigurable devices. However, all these earlier semi-compact mobile and non-industry standard studies still require an expert operator to interpret the results and make reliable decisions based on fault frequency analysis. In addition, the existing monitoring devices are implemented in time based maintenance, or at a certain time interval of data collections (days, months, even years), which may be very late for immediate action to be taken, and the monitoring service can be costly.

Regarding the diagnosis measurement techniques, it has also been observed that a number of commercial condition monitoring devices usually focus on one type of sensor measurement. For example, the condition monitor may employ only a vibration sensor to detect an electrical fault, without considering alternative sensors and methods, although they may be more effective than vibration techniques. Payne et.al $[3,13]$ has indicated that appropriate measurement approaches could lead to comprehensive understanding about the real condition of the motors. Hence, it is important to consider multiple sensor types which can produce better indicators for certain faults which may be combined to obtain accurate results for fault detection [14].

Therefore, it is foreseen that there is a need for a true-compact industry standard condition monitoring system to overcome the limitations present in the existing monitoring systems and services. It is also desirable to have a compact device that can be located next to the machine under test, and which has some form of indicator that can be observed by lay operators. The aim of this research is to develop a compact industrial class of condition monitoring system, which is less expertoriented, which can consider common failures, and which can combine several measurement techniques through multiple sensor signals.

\subsection{Outline of the Thesis}

This thesis is divided into six chapters that can be summarized as followed. Chapter 2 provides a literature review of the main components of condition monitoring systems. Here, a brief comparison of various condition monitoring 
techniques is provided, and specific on-line condition monitoring techniques that were investigated. Data analysis techniques and data processing devices are also reviewed in this thesis. In addition, general CompactRIO-based applications are searched, as this system is the most promising mobile platform for condition monitoring.

In Chapter 3, to provide a good understanding of various faults in induction motors, the theory of induction motors including basic construction and operation principles, is discussed. Then, common faults in induction motor such as stator, rotor, bearing and set-up faults are explained. In this chapter the fault frequency predictors of previous studies are also highlighted. This knowledge provides essential background for the discussion of condition monitoring software that is covered in Chapter 4.

Chapter 4 provides the details of the CompactRIO-based condition monitoring system, including hardware and software development. The first section covers the CompactRIO-based unit, its architecture and custombuilt and/or common modules. The custom-made CompactRIO indicator module is also covered in this chapter. In addition, the FPGA reconfigurable module development and CompactRIO-based condition monitoring analysis software are discussed.

In Chapter 5, the evaluation of the CompactRIO system is undertaken. The chapter discusses the CompactRIO-based execution time evaluation and monitoring of hardware resources. The feature of signal processing application to ease fault frequency determination is also discussed. Finally, the chapter reports the implementation of stator current, voltage, vibration and flux leakage detection methods to identify typical faults in an induction motor under test.

Chapter 6 presents the main conclusion of this research, and provides possible future improvements, which can be based on the CompactRIO architecture. 


\section{Chapter 2}

\section{Literature Review}

\subsection{Introduction}

Many studies have been carried out in the field of condition monitoring of induction motors, including the study of monitoring devices. Considering the objectives highlighted in the previous chapter, this chapter aims to investigate the previous studies to provide background knowledge regarding condition monitoring research suitable for induction motor monitoring. In the following section, various approaches used in condition monitoring system will be discussed as it is important to provide a framework of essential criteria for the proposed condition monitoring system.

\subsection{Approaches used in Condition Monitoring Systems}

Several approaches to development of a reliable condition monitoring system have been reported in the literature. For example, Payne et.al in [13] reported that several aspects need to be considered in order to achieve a reliable condition monitoring system. These aspects are common faults in induction motors, monitoring techniques including sensor requirements, and type of data analysis including monitoring practice. Another study in [15] indicated that to avoid unnecessary maintenance tasks, condition monitoring systems should consider three aspects: data acquisition, data processing and decision making. Furthermore, it has been reported that data processing should be flexible enough to analyze the data both in time domain or in frequency domain. In addition, for a better result, it was considered that decisionmaking steps should be undertaken by both diagnostic action and prognostic action [15]. The authors in [15] have indicated that diagnostic action is concerned with fault detection, isolation and identification, while prognostic action is concerned with fault prediction before faults occur or prediction of the remaining life of a machine. 
Furthermore, Tavner and Penman in [16] highlighted functional tasks or essential aspects that should be considered in a condition monitoring system as illustrated in Figure 2.1 below.

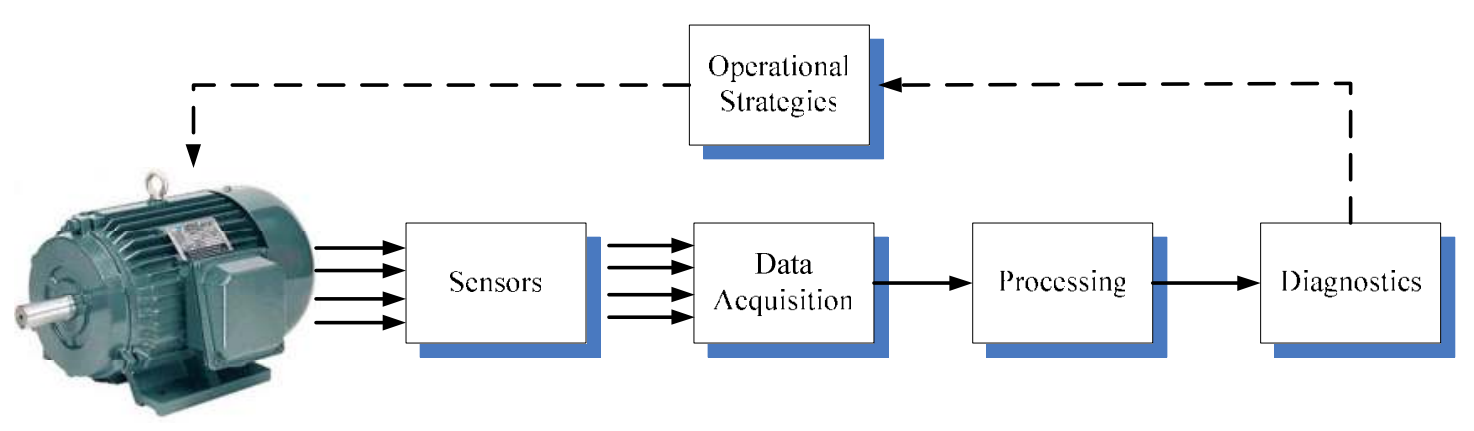

Figure 2.1 Monitoring Tasks [16]

As shown in Figure, four primary tasks should be included in a condition monitoring system. In the first task, sensing tasks are performed, principally to capture any physical quantities produced by motor's fault and to provide outputs in the form of primary quantities that can be recognized by the subsequent task, i.e. data acquisition unit. In addition, Tavner and Penman highlighted that sensing tasks should consider suitable monitoring techniques for the sensor implementations. Moreover, in the sensing stage, a signal conditioning or a pre-amplification may be required. The second task, the data acquisition stage, performs ADC conversion, scaling and storing data. The third stage is the processing task, where offline real data collected in the previous stage are handled and analyzed to obtain better interpretation and understanding. To extract features under an operating data condition this stage includes data signal processing algorithms. In the following stage, fault diagnostic and decision-making tasks can be made based on suitable mathematical models, experiences, standards or other criteria which can influence the operational or maintenance feedback task as shown in Figure 2.1.

It can be summarized from the above discussion that a number of critical design and analysis tasks should be taken into account for the development of an effective condition monitoring system. In addition to the tasks discussed it is important to train the entire system for given motor size loading conditions and faults. Since the aim of this thesis is the development of a compact condition monitoring system, the hardware requirements will be discussed. 


\subsection{Common Faults of Induction Motor}

A fault in an induction motor is commonly interpreted as a condition of decreasing capability in relation to its rated performance [14]. The decrease of the capability may be due to normal wear, poor design, poor mounting (including poor alignment), wrong use, or a combination of these [14]. If a small fault in a motor is not detected in a timely manner, and is allowed to grow further, this may lead to a complete motor failure. Therefore, as stated previously, the condition monitoring strategy needs to be employed to identify faults as early as possible.

As is commonly known, through an implementation of a condition monitoring technique, continuous evaluation of a motor's health is performed to prevent any catastrophic failures during its serviceable life [16]. Such condition monitoring enables operators to take timely action to perform necessary maintenance tasks. In an ideal system, the continuous evaluation should be carried out through the detection of any failure at each specific section of the induction motors. However, the study in [13] indicates that effective monitoring can be done by concentrating only on common failure modes, rather than by covering all potential failures partially. The reason for this is that detection of common classes of failures might reveal the highest probabilities of motor faults. Such classes of failures can provide a simple interpretation of the real condition of the motors, which can be utilized even by lay people. Hence, before the development of fault detection algorithms in a compact condition monitoring system, it is important to first identify the common in faults of the induction motors.

Several valuable survey results were reported in the literature about the common failures of electric motors. Two statistical studies were reported by EPRI and IEEE-IAS in [17, 18], and another one reported in [14] for a specific industry. The result given in Figure 2.2.a and 2.2.b covered about 5000 electrical machines, which 97\% were induction motors. In addition, the results in Figure 2.3.c [14] included 483 induction motors. 


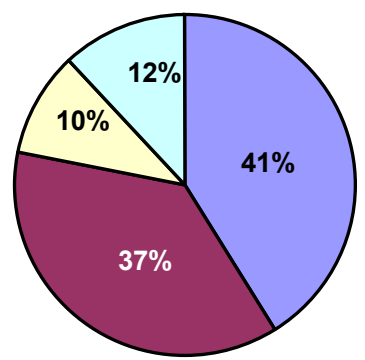

(a)

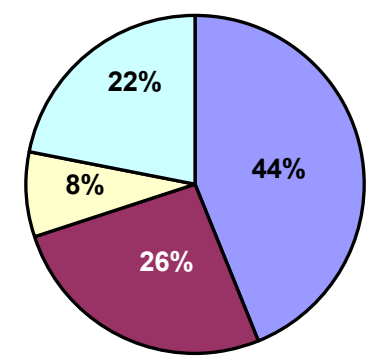

(b)

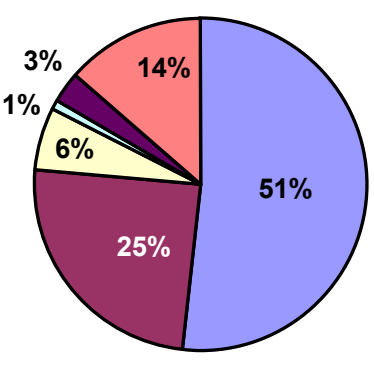

(c)

\begin{tabular}{|lll|}
\hline$\square$ Bearing Related & $\square$ Stator Related & $\square$ Rotor Related \\
$\square$ Other & $\square$ Shaft/coupling & $\square$ External Device \\
\hline
\end{tabular}

Figure 2.2 Distribution of common faults of electric motors according to (a) EPRI survey results (b) IEEE-IAS survey results and (c) survey result in a petrochemical industry

These survey results clearly indicate the bearing-related faults were more than $40 \%$ of the total failures. The EPRI and IEEE-IAS research indicated that stator related faults were the second highest, $37 \%$ and $26 \%$ respectively, rotor-related faults were around $9 \%$, and the other failures were $14 \%$ and $22 \%$. Two additional survey studies in $[19,20]$ also reported that air-gap eccentricity was another common faults in various industries. It was also observed from an experience of industry that most induction motors experience power supply problems such as unbalanced supply voltage, under/over voltage, phase loss, and ground faults [21]. Some of the power supply problems (such as voltage imbalance) were observed as the root cause of many electrical related problems. Therefore, the above mentioned failures, such as bearing, stator, rotor, eccentricity and power supply faults, are considered as the common induction motor fault groups which will be used in this thesis for the development of the condition monitoring system.

\subsection{Monitoring techniques}

Monitoring techniques should be examined carefully to be able to identify potential failure modes. The examination of monitoring techniques is essential in order to categorize which method is the most suitable technique regarding the symptoms of certain failures. In other words, certain methods may be more sensitive in detecting signals of damaged parts than others. The industry experience reported in 
[14] suggested that ultimate and reliable condition monitoring system can be done by combining several condition monitoring techniques.

The condition monitoring techniques studied in the literature can be classified in a number of ways. In [22], 13 monitoring techniques were reported to detect stator related faults which most of them can be implemented for a wide range of induction motor faults. In addition, Thorsen and Dalva [14] also categorized the monitoring techniques implemented in industry, which were classified based on the hardware structure such as, mechanical and electromechanical. Figure 2.3 illustrates summary of the monitoring techniques reported in the literature.

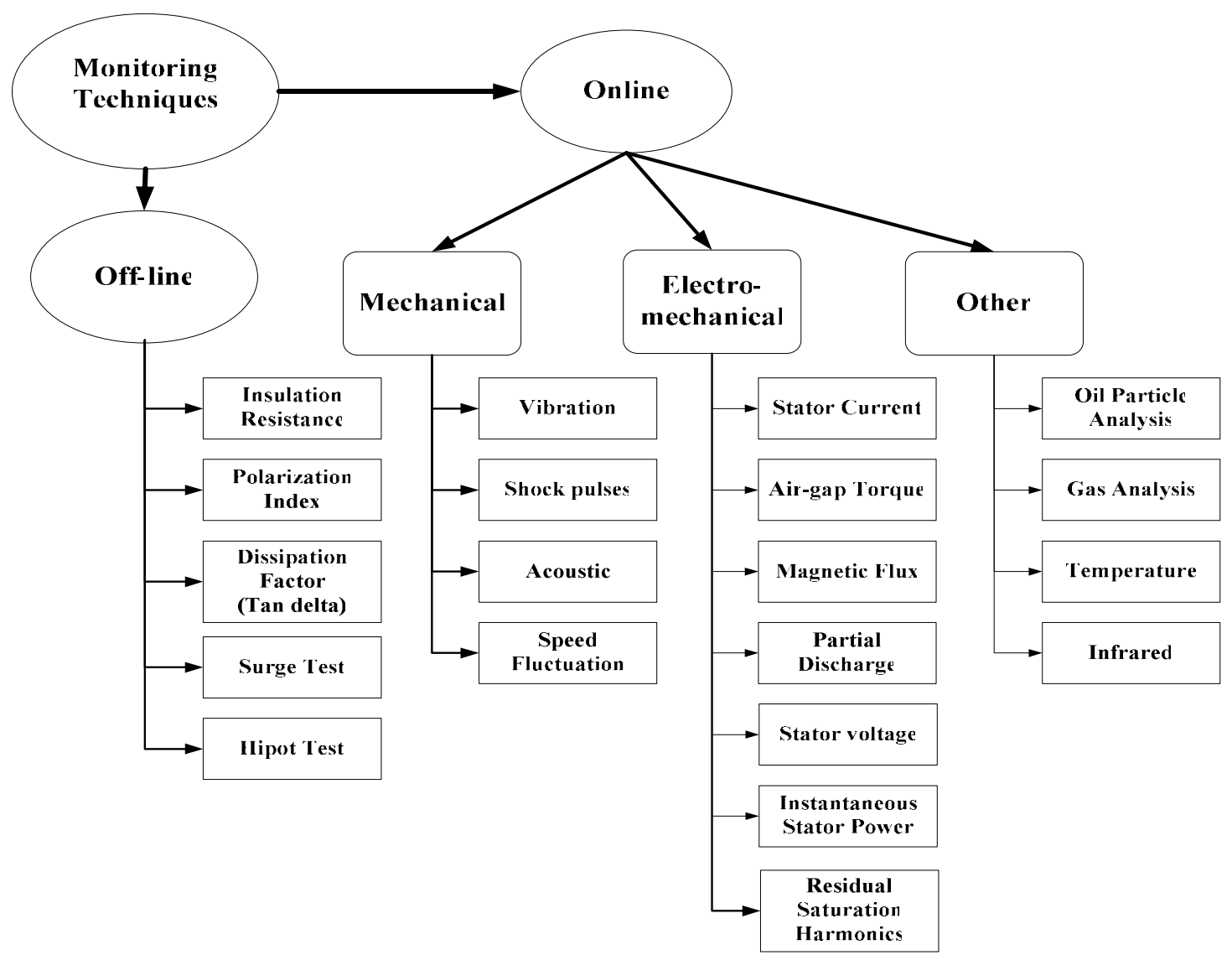

Figure 2.3 Condition monitoring of induction motors

As shown in Figure above, the monitoring techniques can be classified into two major groups: off line and on-line methods. As the name suggests the on-line monitoring is suitable while the motor is operating. In the case of the off line method, however, the motor should be stopped. Table 2.1 and Table 2.2 are given to briefly describe these on-line and off line monitoring techniques of including their advantages and disadvantages. Special techniques such as temperature monitoring and chemicalrelated techniques are not discussed in this thesis. 
Table 2.1 Off line condition monitoring techniques

\begin{tabular}{|c|c|c|c|}
\hline $\begin{array}{l}\text { Monitoring } \\
\text { Techniques }\end{array}$ & Descriptions & Advantages & Disadvantages \\
\hline $\begin{array}{l}\text { Insulation } \\
\text { Resistance } \\
\text { (IR), } \\
\text { Polarization } \\
\text { Index (PI), } \\
\text { Dissipation } \\
\text { Factor } \\
\text { (Tan Delta), } \\
\text { [23] }\end{array}$ & $\begin{array}{l}\text { Ratio of DC voltage applied } \\
\text { between copper winding and } \\
\text { ground (IR), Ratio of } 10 \\
\text { minute reading over } 1 \text { minute } \\
\text { reading of IR (PI), Tangent of } \\
\text { angle between measured } \\
\text { electric current of capacitive } \\
\text { component of electrical } \\
\text { insulation material and leakage } \\
\text { current of insulation material } \\
\text { (Tan Delta) }\end{array}$ & $\begin{array}{l}\text { - Low-cost equipment } \\
\text { - Useful to recognize severe insulation } \\
\text { problems because of thermal } \\
\text { deterioration, moisture absorption, or } \\
\text { end winding contamination (IR) } \\
\text { - Detects how dry and clean the winding } \\
\text { (PI) is } \\
\text { - Provides an indication about the } \\
\text { dielectric losses and aging of stator } \\
\text { insulation (Tan Delta) }\end{array}$ & $\begin{array}{l}\text { - Motor must be turned off and invasive } \\
\text { technique. Testing cannot be performed } \\
\text { frequently enough (typically once every three } \\
\text { to six years) } \\
\text { - Not sensitive to internal winding insulation } \\
\text { condition } \\
\text { - Very temperature-sensitive } \\
\text { - Difficult to obtain identical measurement } \\
\text { conditions as the result are affected by the } \\
\text { environmental conditions }\end{array}$ \\
\hline Surge Test [24] & $\begin{array}{l}\text { Comparing two returning } \\
\text { waveforms of identical high } \\
\text { voltages pulses which are } \\
\text { imposed on two phases of the } \\
\text { motor windings with third } \\
\text { phase is grounded }\end{array}$ & $\begin{array}{l}\text { - Cost-efficient, and reliable } \\
\text { - Useful for stator insulation and } \\
\text { eccentricity } \\
\text { - Minimum noise interference }\end{array}$ & $\begin{array}{l}\text { - } \text { Motor must be off } \\
\text { - Invasive method }\end{array}$ \\
\hline
\end{tabular}


Table 2.1 Off line condition monitoring techniques (continued)

\begin{tabular}{|c|c|c|c|}
\hline Monitoring & Descriptions & Advantages & Disadvantages \\
\hline $\begin{array}{l}\text { AC/DC Stator } \\
\text { Hipot Test [26] }\end{array}$ & $\begin{array}{l}\text { Measuring an AC voltage } \\
\text { applied between all the leads in } \\
\text { parallel and ground at a } \\
\text { voltage level equal to twice the } \\
\text { rated value plus } 1000 \mathrm{~V} \text {. } \\
\text { In the case of dc voltage, the } \\
\text { voltage applied should be } 1.7 \\
\text { times the ac value }\end{array}$ & $\begin{array}{l}\text { Useful to prove the integrity of the stator } \\
\text { insulation system of a complete motor }\end{array}$ & $\begin{array}{l}\text { - Can be destructive to the insulation system so } \\
\text { it should not be performed excessively } \\
\text { - Can be risk to operators } \\
\text { - DC Hipot has higher margin of safety than an } \\
\text { AC Hipot test }\end{array}$ \\
\hline
\end{tabular}

Table 2.2 On-line condition monitoring techniques

\begin{tabular}{|c|c|c|c|}
\hline Monitoring & Description & Advantages & Disadvantages \\
\hline $\begin{array}{l}\text { Residual } \\
\text { Saturation } \\
\text { Harmonics[27] }\end{array}$ & $\begin{array}{l}\text { Measuring harmonic } \\
\text { frequencies of stator induced } \\
\text { voltage after a motor is } \\
\text { switched-off }\end{array}$ & $\begin{array}{l}\text { - Useful to determine stator faults. } \\
\text { - Does not require motor parameters } \\
\text { - Unaffected by voltage imbalance and } \\
\text { stator currents } \\
\text { - Faulty phase can be identified }\end{array}$ & Motor must be turned off \\
\hline Vibration [28] & $\begin{array}{l}\text { Fault of certain parts in } \\
\text { machines generates specific } \\
\text { vibration spectrum which can } \\
\text { be measured by vibration } \\
\text { sensors }\end{array}$ & $\begin{array}{l}\text { - Sensors can be non-invasive or invasive } \\
\text { - The most developed technique. It is } \\
\text { covering most of the faults } \\
\text { - More sensitive to mechanical-related } \\
\text { faults (bearing, mechanical unbalance, } \\
\text { misalignment, and soft foot) }\end{array}$ & $\begin{array}{l}\text { - Expensive sensors } \\
\text { - Less sensitive to electrical-related faults } \\
\text { - Sensitive to mobility of machine } \\
\text { - Effect of attenuation of path from a vibration } \\
\text { source to sensors } \\
\text { - Effect of accelerometer characteristic (no true } \\
\text { DC response, ground loop problems, } \\
\text { mounting surface problems and cable length } \\
\text { sensitivities) } \\
\text { - Load sensitive }\end{array}$ \\
\hline
\end{tabular}


Table 2.2 On-line condition monitoring techniques (continued)

\begin{tabular}{|c|c|c|c|}
\hline Monitoring & Description & Advantages & Disadvantages \\
\hline $\begin{array}{l}\text { Shock Pulses } \\
\text { [29] }\end{array}$ & $\begin{array}{l}\text { Detecting mechanical } \\
\text { ultrasonic shock generated by } \\
\text { contact of damage ball bearings } \\
\text { with the raceways of bearing or } \\
\text { debris, or vice versa }\end{array}$ & $\begin{array}{l}\text { - Excellent for ball bearing monitoring } \\
\text { - Can be invasive or non invasive } \\
\text { - Need simple signal processing (time } \\
\text { domain analysis) }\end{array}$ & - Sensitive to direct modulation \\
\hline $\begin{array}{l}\text { Acoustic } \\
\text { Emission } \\
\text { Monitoring } \\
{[30,31]}\end{array}$ & $\begin{array}{l}\text { Monitoring elastic waves which } \\
\text { is produced spontaneously } \\
\text { within a material under stress } \\
\text { (audible or ultrasound) }\end{array}$ & $\begin{array}{l}\text { - Particularly promising for } \\
\text { bearing/mechanical related faults } \\
\text { - Non invasive } \\
\text { - Easy to set up microphone (non-contact } \\
\text { sensors) }\end{array}$ & $\begin{array}{l}\text { - Source of faults can be easily attenuated } \\
\text { (airborne acoustic monitoring) } \\
\text { - Might be difficult to be implemented in } \\
\text { environment with noisy backgrounds } \\
\text { - Difficult to interpret the signals }\end{array}$ \\
\hline $\begin{array}{l}\text { Stator Current } \\
{[4,32,33]}\end{array}$ & $\begin{array}{l}\text { Sensing electrical signal of } \\
\text { motor supply currents, which } \\
\text { contain a direct by-product of } \\
\text { rotating flux components } \\
\text { caused by specific faults }\end{array}$ & $\begin{array}{l}\text { - Covers almost all faults (currently is the } \\
\text { most popular technique) } \\
\text { - Non-invasive } \\
\text { - More sensitive to electrical-related } \\
\text { faults (stator, rotor, and eccentricity } \\
\text { problems) } \\
\text { - Low-cost system }\end{array}$ & $\begin{array}{l}\text { - A possible misinterpretation of sideband } \\
\text { frequencies with the motor current } \\
\text { modulation produced by other events } \\
\text { - In large motors, a spidered structure tends to } \\
\text { produce a magnetic asymmetry whose effect } \\
\text { is similar to rotor electrical asymmetry } \\
\text { - Influenced by electric supply, static and } \\
\text { dynamic load conditions, noise, motor } \\
\text { geometry, and fault conditions }\end{array}$ \\
\hline $\begin{array}{l}\text { Flux } \\
\text { Monitoring } \\
\text { (search coils } \\
\text { in stator or } \\
\text { around shaft) } \\
\text { [20] }\end{array}$ & $\begin{array}{l}\text { Monitoring small part of } \\
\text { sinusoidal leakage flux, which } \\
\text { can be affected by various } \\
\text { asymmetries and fault } \\
\text { conditions }\end{array}$ & $\begin{array}{l}\text { - Can be used to wide rage of fault } \\
\text { conditions } \\
\text { - Can be invasive (in stator) or less } \\
\text { invasive (around shaft) } \\
\text { - Transducers do not need isolation or } \\
\text { protection against current/voltage }\end{array}$ & $\begin{array}{l}\text { - Sometimes not practical to install search coil } \\
\text { for large motors } \\
\text { - Output voltage drift (temperature sensitive) } \\
\text { - Very load-dependant } \\
\text { - Flux can be easily attenuated by frame for a } \\
\text { complex steel frame of large motor (axial } \\
\text { search coil) }\end{array}$ \\
\hline
\end{tabular}


Table 2.2 On-line condition monitoring techniques (continued)

\begin{tabular}{|c|c|c|c|}
\hline Monitoring & Description & Advantages & Disadvantages \\
\hline $\begin{array}{l}\text { Voltage } \\
\text { Monitoring } \\
{[34]}\end{array}$ & $\begin{array}{l}\text { Monitoring of stator voltages, } \\
\text { which may contain valuable } \\
\text { line frequency or other } \\
\text { information }\end{array}$ & $\begin{array}{l}\text { - Non-invasive } \\
\text { - Providing valuable information for air gap } \\
\text { torque and instantaneous power monitoring } \\
\text { - Unaffected by motor drive system }\end{array}$ & $\begin{array}{l}\text { Lack of publications presenting this } \\
\text { monitoring technique }\end{array}$ \\
\hline $\begin{array}{l}\text { Air Gap } \\
\text { Torque [35] }\end{array}$ & $\begin{array}{l}\text { Gained from effect of } \\
\text { combination current (both rotor } \\
\text { and stator) voltage and flux } \\
\text { linkages }\end{array}$ & $\begin{array}{l}\text { - Currently specific for rotor-related fault } \\
\text { monitoring } \\
\text { - Sensitive to unbalance defect and unbalance } \\
\text { voltage }\end{array}$ & $\begin{array}{l}\text { - The computation of the air-gap torque } \\
\text { needs a machine model } \\
\text { - Lack of publication and standards }\end{array}$ \\
\hline $\begin{array}{l}\text { Instantaneous } \\
\text { Stator Power } \\
{[36-38]}\end{array}$ & $\begin{array}{l}\text { Product of supply voltage and } \\
\text { current that are affected by } \\
\text { phase shift. }\end{array}$ & $\begin{array}{l}\text { - Can be used to detect broken rotor bar faults, } \\
\text { rotor cage fault, eccentricities, bearing and } \\
\text { interturn short circuits in the stator winding } \\
\text { - Additional components in low frequencies } \\
\text { might improve fault detections } \\
\text { - Easy to filter the dc component in the power } \\
\text { spectrum } \\
\text { - Mixed faults can be separated clearly (no- } \\
\text { cross terms) }\end{array}$ & $\begin{array}{l}\text { - Sensor might be costly because of the } \\
\text { implementation of monitoring using six } \\
\text { electrical signals (three phase currents } \\
\text { and three phase voltages) }\end{array}$ \\
\hline $\begin{array}{l}\text { Partial } \\
\text { Discharge } \\
{[25,39]}\end{array}$ & $\begin{array}{l}\text { Sensing small electrical sparks, } \\
\text { which occur as a result of } \\
\text { insulation imperfections }\end{array}$ & $\begin{array}{l}\text { - Useful for stator insulation assessment for } \\
\text { more than } 4 \mathrm{kV} \text { motors } \\
\text { - Providing assessment of winding residual life } \\
\text { - Test can be performed more frequently than } \\
\text { off line } \\
\text { - Loose coils in stator slot can be detected }\end{array}$ & $\begin{array}{l}\text { - Sensitive to electrical interference } \\
\text { - Significant expertise to separate PD } \\
\text { signals from noise is required }\end{array}$ \\
\hline
\end{tabular}


Shown in these tables that, generally, online techniques provide assessment of the condition of induction motors without stopping their operation. In other words, implementation of the online techniques does not need to interrupt plant production. Online techniques are also a promising way for operators to obtain real-time data, which can be used to observe the current condition of induction motors. In addition, most of on-line techniques offer multiple fault recognitions, non-invasive and safe. The most important point is that the online techniques allow frequent data capturing and analyzing which is valuable for understanding the trend of the induction motor condition. It can be summarized that on-line techniques present advantages in many ways when motors are required to be operated continuously, without damaging any parts.

Considering how to select the most suitable condition monitoring application, Thomson in [4] suggested a number of factors such as:

1. Sensor should be non-invasive

2. Sensor and instrumentation system must be reliable

3. The diagnosis must be reliable

4. The severity of the problem should be quantified

5. Ideally, an estimation of remaining run-life should be given

6. Ideally, prediction of the fundamental cause of problems should be provided via on-line information.

According to Thomson, in many cases it was possible to achieve criteria 1) to 4), but was difficult to achieve criteria 5) and 6). These factors considerably introduce several types of online condition monitoring techniques to be employed in the development of a condition monitoring system.

Among several types of online condition monitoring techniques, vibration monitoring, stator current monitoring, flux monitoring (axial external search coil), voltage monitoring are the suitable techniques to be implemented. Most of these techniques meet the first four factors suggested for condition monitoring. These selected monitoring techniques are non-invasive and detect a wide-range fault of induction motors. These selected online monitoring techniques have been widely implemented using reliable sensors and instrumentations. Furthermore, several studies have demonstrated the diagnosis capabilities of these techniques including the severity level quantifications presented in the following table. 
It should be noted that instantaneous power is not considered in this thesis, even though it has promising capabilities. The reason is that, in this research, the author considers basic the parameters to be measured, such as current, or vibration, and not the derivate parameters, which need to be combined or added with some mathematical models.

Table 2.3 Studies of common faults in induction motors using vibration, stator current, stator voltage and flux leakage monitoring

\begin{tabular}{|c|c|c|c|c|}
\hline Fault $\quad$ Monitoring & Vibration & Stator Current & $\begin{array}{c}\text { Stator } \\
\text { Current/ } \\
\text { Voltage }\end{array}$ & Flux Leakage \\
\hline \multicolumn{5}{|l|}{ Bearing Related } \\
\hline Inner Raceway & {$[28,40-42]$} & [43-45] & & \\
\hline Outer Raceway & {$[28,40-42,46]$} & [43-46] & & \\
\hline Loose Bearing & {$[28,42]$} & [45] & & \\
\hline Cage Fault & {$[28,40]$} & {$[44,45]$} & & \\
\hline Ball Fault & {$[28,41,42]$} & {$[43,44]$} & & \\
\hline Bearing Faults & & [47] & & [48] \\
\hline \multicolumn{5}{|l|}{ Stator Related } \\
\hline $\begin{array}{l}\text { Shorted Turn or } \\
\text { Shorted Coil in } \\
\text { Stator Winding }\end{array}$ & {$[17,28]$} & {$[4,49,50]$} & {$[51]$} & {$[48,52,53]$} \\
\hline Phase to Phase & [17] & & & \\
\hline Stator Loose Iron & [17] & & & \\
\hline \multicolumn{5}{|l|}{ Rotor } \\
\hline $\begin{array}{l}\text { Crack Rotor Bar, } \\
\text { Broken Rotor Bar, } \\
\text { Broken End-Ring in } \\
\text { Rotor Cage }\end{array}$ & {$[17,28,30,54]$} & {$[49,55-57]$} & {$[34,51,58]$} & {$[53,59]$} \\
\hline $\begin{array}{l}\text { Shorted Rotor } \\
\text { Lamination }\end{array}$ & [17] & & & \\
\hline Rotor Asymmetry & & {$[33,60,61]$} & & {$[48,59,62]$} \\
\hline Loose Rotor Bar & {$[17,54]$} & & & \\
\hline Rotor Rub & {$[17,54]$} & & & \\
\hline \multicolumn{5}{|l|}{ Eccentricities } \\
\hline Static Eccentricities & & {$[19,20,63]$} & & [64] \\
\hline $\begin{array}{l}\text { Dynamic } \\
\text { Eccentricities }\end{array}$ & & {$[19,20,63]$} & & [64] \\
\hline $\begin{array}{l}\text { Mixed } \\
\text { Eccentricities }\end{array}$ & {$[17,28,54]$} & {$[19,20,63]$} & {$[58]$} & {$[62,64]$} \\
\hline \multicolumn{5}{|l|}{ Set-up } \\
\hline $\begin{array}{l}\text { Mechanical } \\
\text { Unbalance }\end{array}$ & {$[7,12,17,54]$} & [65] & & \\
\hline Misalignment & {$[7,12,40,54]$} & {$[65]$} & & [48] \\
\hline Soft-Foot & {$[7,12,17,54]$} & [65] & & \\
\hline
\end{tabular}


As mentioned in the previous section, condition monitoring techniques in this thesis will consider common failures of induction motors. In order to obtain comprehensive understanding about the capability of the selected monitoring techniques, Table 2.3 highlights common failures of induction motors which have been studied following each of monitoring technique. It can be observed from the table that the capability of detecting various faults of induction motors of each monitoring method has been explored. Details of these methods will be presented in the next sections.

\subsubsection{Vibration Monitoring}

The vibration detection technique is undoubtedly the most developed technique among commercial condition monitoring systems. Vibration monitoring measures any cyclic excitation force in induction motors due to any wear or failures. As one of the earliest tools in condition monitoring, the vibration monitoring technique covers many principal areas in condition monitoring of induction motors. According to Tavner and Penman [16], the principal areas of vibration include:

- response of the stator core to the attractive force between rotor and stator;

- response of the stator end winding to the electromagnetic forces on conductors;

- dynamic behaviour of the rotor; and

- response of the shaft bearings to vibration transmitted from rotor.

Finley et.al [54], and, Singh and Al Kazzaz [17], also reported a comprehensive list of vibration frequency components due to mechanical or electrical faults. In addition, several vibration based monitoring approaches have been established and widely implemented in commercial condition monitoring equipment, such as overall level monitoring, 1/3 octave spectrum monitoring, frequency spectrum monitoring, and Spectrum analysis [16].

However, compared with mechanical-related faults, there are only few papers discussing vibration monitoring in relation to electrical related faults. Most of these papers are still in the stage of explaining the relationship between electrical faults and vibration signals. For example, Dorrell et.al. put forward a theoretical analysis of the force between stator and rotor because of eccentric conditions, which may be related to specific low fault frequencies using vibration monitoring. Another example of 
vibration study, Chow and Fei [66] demonstrated identification and analysis methods to determine machine asymmetrical faults, stator winding related faults, and their significant relation to vibration. Furthermore, Taylor [28] indicated that the primary difficulties in recognizing electrical-related faults through vibration monitoring technique are because of the complexity interaction between vibrations of mechanical and electrical force. Therefore, it is almost impossible to obtain the real cause of electrical-related faults. For instance, the frequencies of electrical motor problems, which are usually concentrated near line frequency, need considerable effort to be recognized due to superimposing or having a close location with the second harmonic of fundamental rotor or shaft frequency at 4-poles of induction motors.

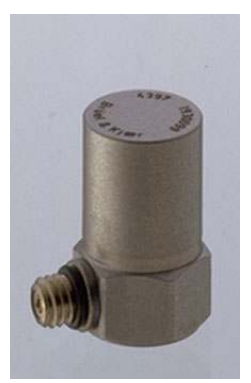

(a)

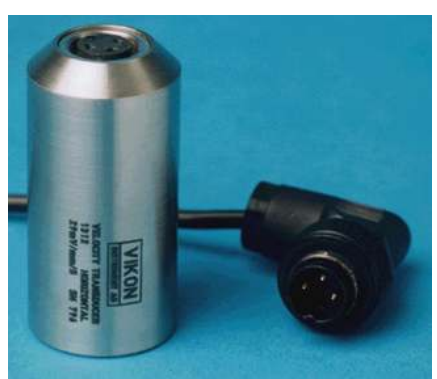

(b)

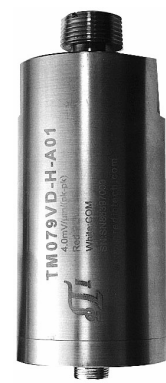

(c)

Figure 2.4 (a) Accelerometer [67], (b) velocity [68], and (c) displacement [69] transducers for vibration monitoring

Vibration measurement using accelerometer, displacement or velocity transducers as shown in Figure 2.4 should be conducted carefully. This is because of the weaknesses of accelerometer characteristics, such as high impedance output, no true DC response, ground loop problems, mounting surface problems and cable length sensitivities [70]. Furthermore, the effect of the transmission path from the source of vibration to the sensors is a major concern in the applications [30]. In other words, the true characteristic of vibration sources can be easily attenuated or interfered with the other materials or other vibration sources. In addition, the vibration monitoring is also sensitive to mobility or flexibility of the machine [16]. Therefore, caution in vibration measurement using an accelerometer should be exercised especially from adjacent, coupled or other near-vibration sources. These vibration sources may generate beat frequencies or slightly change the natural vibration of the real faults. 


\subsubsection{Stator Current Monitoring}

The capabilities of stator current monitoring as a promising approach to replace or complement vibration monitoring have been proposed in many studies. Stator current monitoring is simpler and less intrusive than the vibration technique, as the technique does not require delicate/expensive transducers or direct access to the motor's site. Motor stator current monitoring can be done by sensing electrical signals of the motor supply currents, caused by asymmetrical related problems or specific motor faults. In other words, magnetic flux due to any faults will induce current components in the stator winding, causing modulation of the motor supply current. This modulation frequency of the motor supply current can be extracted to identify each fault. Furthermore, this current measurement can be done easily by fitting a clipon-current transformer around a cable of motor power supply. Figure 2.5 shows transducers which can be installed to detect induction motor faults, based on stator current monitoring.

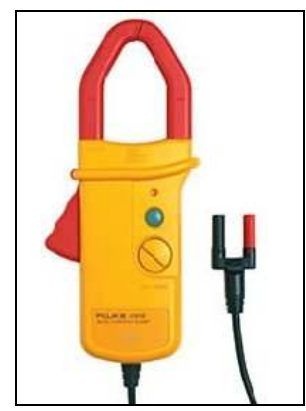

(a)

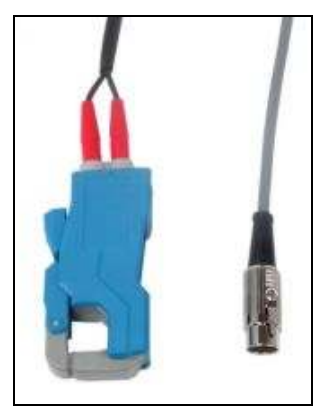

(b)

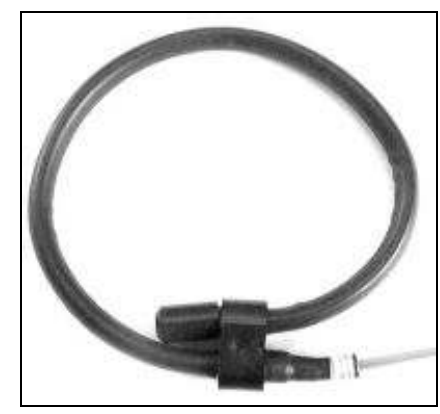

(c)

Figure 2.5 An example of (a) Hall-effect sensor [71], (b) CT sensor [72], and (c) Rogowski coil [73] for stator current monitoring

As listed in Table 2.3, many studies have proved the versatile ability of stator current to detect various faults in induction motors. Several reviews $[22,74]$ have also highlighted the stator current monitoring capabilities in induction motor condition monitoring. In practical applications, the stator current monitoring technique can also be successfully applied through an on-line system for large motors [33] as well as small motors [20] in various industries. 
However, in terms of bearing fault analysis, many studies have underlined several difficulties:

- One study pointed out that stator current monitoring is less sensitive recognizing mechanical defects compared with vibration monitoring [46].

- A bearing fault study in [43] was carried out extensively, where the bearing damages were created by drilling the ball bearing, inner ring and outer ring. In other words, compared with vibration monitoring where the early stage of bearing damage can be identified, stator current monitoring capabilities regarding this fault were not proved, and might even be difficult to pinpoint.

- High magnitude ratio of the supply frequency and bearing defects [43] could lead to a significant problem in peak detection, even though in [75] this ratio problem was overcome by applying a digital/analog cancelling technique.

- Because of modulation frequencies among bearing defects [28], in real cases the bearing defect frequencies may be more difficult to be extracted because the bearing modulated frequencies could be modulated by the stator harmonic frequencies modulation.

- Unlike vibration monitoring through accelerometers, with stator current monitoring it is probably difficult to differentiate which one of the two bearings in an induction motor contributes most to the defect frequencies.

Regardless of the limitations mentioned above, due to the simplicity and also the practicality, many studies suggest that the stator current is believed to be replacing vibration method to detect electrical-related faults [3]. The stator current monitoring, as described earlier, is a promising method which is also powerful in detecting stator winding faults, rotor related faults, and eccentricity. However, Thomson in [4] suggested that predicting accurate slip frequency for the wide range of motor types at different load condition using only a stator current analysis through a continuous monitoring system should include an intelligent diagnosis technique. This means that the prediction of this accurate slip frequency is not an easy task. The prediction slip frequency is essential to determine accurate twice slip frequency sidebands which are required for several fault frequency predictors [4]. It should also be noted that mechanical load torque oscillations, voltage fluctuations and bearing damages could overlap the position of twice slip frequency sidebands, causing misinterpretation of 
real fault signals [4]. Therefore, monitoring analysis using this technique requires careful interpretation.

\subsubsection{Stator Voltage Monitoring}

Compared with other monitoring techniques, stator voltage monitoring is rarely chosen as an independent monitoring technique. In most cases, this monitoring technique is combined with stator current monitoring, allowing for another type of monitoring technique such as instantaneous stator power [36] and air gap torque [35]. Stator voltage monitoring is sometimes used to confirm specific fault frequencies of an induction motor, obtained from analysis of stator current monitoring [65]. Regardless of the potential capabilities as listed in Table 2.3, stator voltage monitoring might be used to provide valuable information to confirm certain faults, or to provide exact fundamental line frequency of an induction motor.

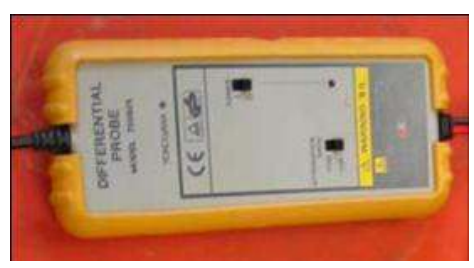

Figure 2.6 A differential voltage measurement device for voltage monitoring [76]

Similar to the stator current monitoring method, this technique can be implemented by measuring stator voltage. Figure 2.6 shows a differential voltage transducer, which can be used to measure voltage through the stator line supply.

\subsubsection{Flux Leakage Monitoring}

Another monitoring method is flux leakage monitoring. This monitoring method is a general-purpose detection method, which can also show various types of faults using only one sensor. This technique can be carried out by detecting small parts of the total flux leakage both from the rotor and stator sides [48] as illustrated in Figure 2.7. Ideally, due to symmetrical nature of flux of a healthy ideal machine on both rotor and stator sides, the total flux across the air gap radially can be considered zero [77]. However, the reality is that no machines are constructed perfectly symmetrical. Consequently, a small leakage flux will exist even in healthy motors. Therefore, a basic idea of this monitoring technique is that a small failure of the induction motor causes asymmetry, which in turn increases the existent flux leakage 
[77]. This flux leakage appears as flux frequency spectrum through a monitoring system.

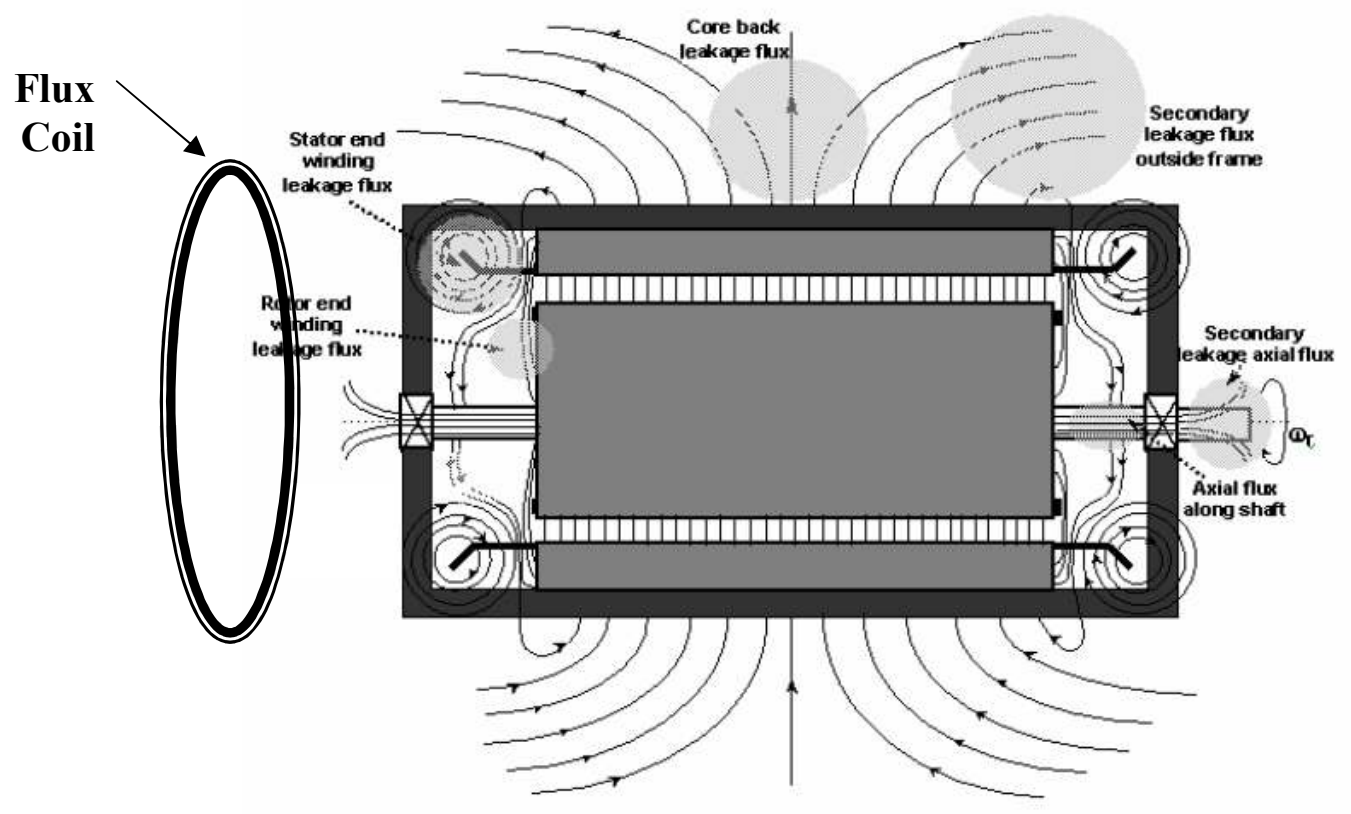

Figure 2.7 Flux region which can be used to detect fault in induction motors [4]

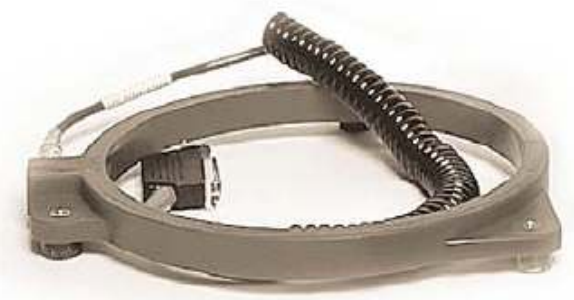

\section{Figure 2.8 A commercial external flux coil for flux leakage monitoring [78]}

The technique of monitoring flux leakage is relatively straightforward and simple. It can be done by measuring flux leakage at certain spots. For example, as can be seen in Figure 2.7, flux leakage of the stator end winding and rotor end winding are the main cause of the flux leakage at the axial position (known as axial flux leakage). Hence, to observe axial flux leakage, particular coils are applied in these flux spots. Two basic types of coils can be selected, using an external coil wound around the shaft of the motor (axial direction), as shown in Figure 2.8, or an internal axial flux coil (airgap search coil) [53]. External coils are preferable because of their noninvasive characteristics. This type is also easy to install for a new or an operating motor.

Because flux leakage is affected by any change in the space harmonic distribution in the air gap due to any faults, it is claimed in [53] that flux leakage 
monitoring is capable of identifying various faults. These faults are broken rotor bars, stator winding interturn short circuits, eccentric running, wound rotor short circuit, loss of phase and negative phase sequence in supply lines. Penman et. al. [52] demonstrated the flux leakage method capability of identifying not only the shorted turn of the stator windings but also the fault locations. In addition, Cabanas et. al [48] also evidenced several experimental records of flux leakage application, including broken rotor bar faults, shaft misalignments, interturn short detection, and bearing damage detection. Interestingly, one study also reveals that flux leakage monitoring is more sensitive compared with the stator current to detect broken damper bar or shortcircuited turns in power generator rotor windings [79]. This means that this technique might be better than the stator current technique if the weaknesses can be overcome.

Despite the potential capabilities, because of the weaknesses as listed in Table 2.2, flux leakage monitoring is not as popular as stator current monitoring. For example, sometimes fault frequencies of axial flux leakage can be more difficult to be identified in a large motor because of flux attenuation of the complex frames. Furthermore, similar to stator current monitoring, this method is load dependant. A study [48] has also pointed out the lack of mathematical models describing the effect of motor failures on the axial flux spectrum.

It can be summarized from the techniques reviewed above that each condition monitoring technique might not provide a similar result in relation to each fault. For example, vibration monitoring seems to be the best method to detect mechanical problems such as bearing and set-up faults. However, stator current monitoring, can be an effective method for several electrical-related problems, such as stator faults, eccentricity faults, and broken rotor bar faults. Therefore, it is suggested that a combination of three or four online monitoring techniques may provide a better solution in identifying various faults.

\subsection{Data Analysis and Diagnostics}

Critical approach to identify the fault frequencies should accommodate an appropriate data analysis and diagnostics task. As mentioned in the previous section that data analysis is part of processing task as shown in Figure 2.1. The data analysis converts raw digital data into other forms that can be easily interpreted. Furthermore, 
based on the output of data analysis, diagnostics tasks are sometimes performed to suggest the current condition of induction motors, and even to recommend action for further maintenance. This data analysis can be carried out in different forms. For example, a simple RMS amplitude, wavelet analysis, spectrum analysis, and FFT (signal processing-based). In addition, the diagnostic tasks can be in a simple method such as threshold and enveloping, or an advanced method such as complex artificial intelligence and expert systems.

It should be noticed that this section focuses on the FFT-based signal processing technique and threshold as the simplest, compared with some other techniques. Moreover, threshold is not discussed further in this literature review but it will be explained in the system development chapter.

Various signal processing techniques have been implemented in the motor condition monitoring devices [80], such as FFT, instantaneous power FFT [36], bispectrum or high order spectra [66, 81, 82], high resolution spectra analysis [83] and wavelet analysis [44, 45, 84]. Each of these methods offers varying advantages, however, each of them also has inherent disadvantages. For example, the bispectrum method offers precise results with Gaussian noise suppression, but the data analysis has a high computational overhead.

As mentioned in the Introduction, FFT still dominates most of the condition monitoring implementation in industry and research, even though it has limitations. The reason for its implementation is that FFT has a simple technique compared with the other systems. However, it is well known that FFT analysis is designated only for steady state signals $[80,85]$. FFT analysis sometimes fails to detect bearing damage because the low energy of the bearing damages is usually overwhelmed with noise [45]. To overcome these problems, especially in relation to noisy data, several complementary techniques are usually applied such as averaging, smoothing, the peak hold method, enveloping and using inverse FFT [41, 85]. Furthermore, in the engineering approach, the motor vibration signals are usually considered stationary in the relatively short data acquisition intervals and assumed in the steady state operations. Therefore, FFT analysis has become very popular in condition monitoring over the last thirty years. 


\subsection{Condition Monitoring Devices}

As stated in the first section of this chapter a complete structure of condition devices is one of the main concerns in this thesis. Illustrated in Figure 2.9 are data acquisition and processing main tasks consisting of detailed hardware tasks. For example, a data acquisition task may contain a multiplexer, a sample and hold function, which can be built from a high speed amplifier, and an Analog to Digital Converter (ADC).

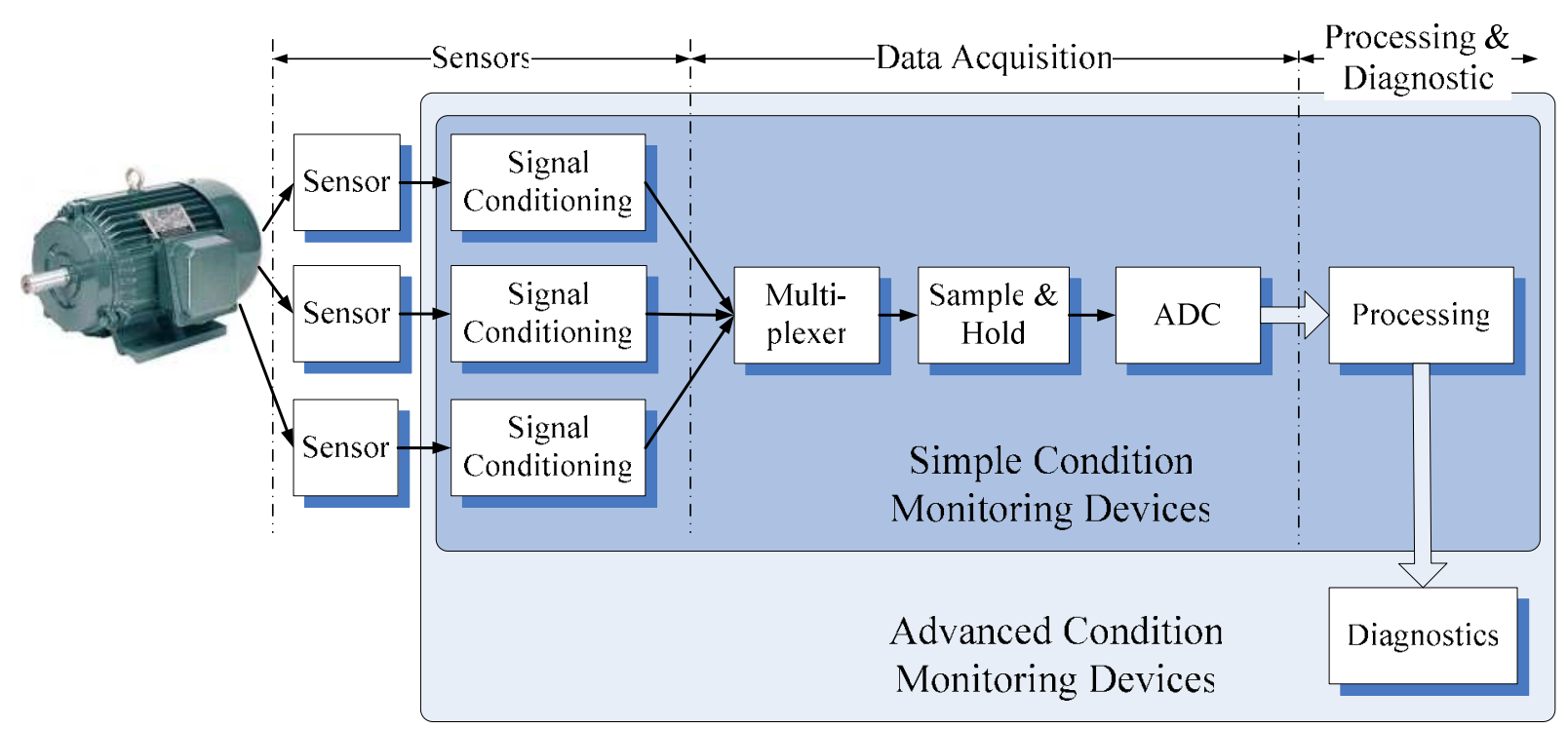

Figure 2.9 A complete structure of a condition monitoring device [16]

An important point shown in Figure 2.9 is the difference between simple and advanced condition monitoring devices. The simple condition monitoring devices may not cover all tasks, such as the diagnostic function. In addition, for the advanced devices, advanced decision-making is usually adopted. Presentation functions such as an LCD or simple LEDs indicators are usually added at the end of functional block to communicate the results with operators. Based on this basic structure shown in Figure 2.10, various condition monitoring devices have been developed as a foundation for the implementation of condition monitoring-based maintenance.

However, the condition monitoring devices are a dilemma for consumers to reach a balance between achieving condition monitoring objectives, and the cost allocated for the devices. As described in the Introduction, several advanced devices which are required for effective monitoring may be expensive. However, commercial 
handheld devices are expert-oriented. Examples of these commercial condition monitoring devices are illustrated in Figure 2.10.
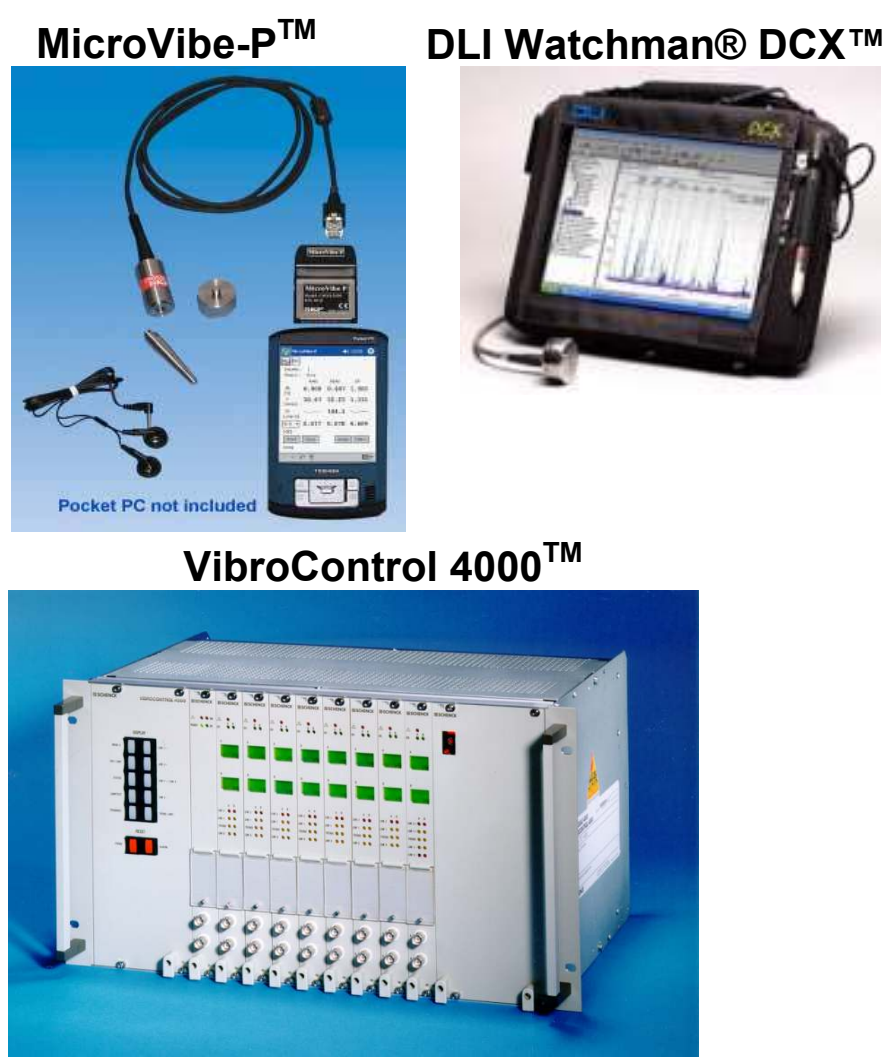
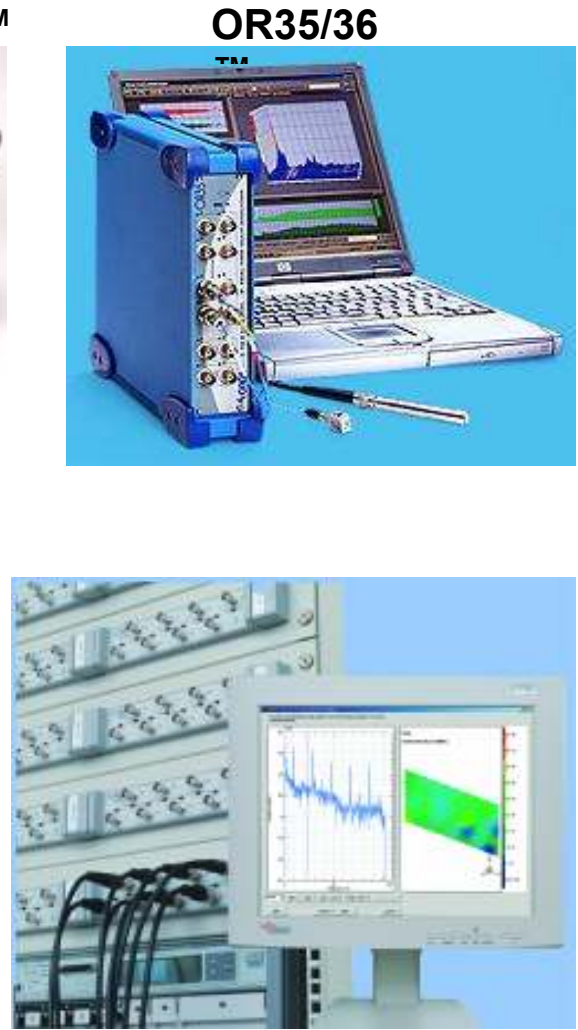

Figure 2.10 Commercial handheld (MicroVibe-P), portable (DLI Watchman and OR35/36), and permanent on-line (VibroControl 4000 and VXI SO analyzer) condition monitoring devices

Shown in Figure 2.11, on-line condition monitoring devices can be divided into handheld, portable and permanent on-line types [7]. Permanent on-line devices are implemented where the data are continuously captured, analyzed, stored and presented. These devices are dedicated permanently for monitoring and providing an early warning evaluation for operators from a special control room or a certain location near the induction motors. Therefore, these examples of permanent on-line equipment are sometimes fitted with Local Area Network (LAN) communication. Handheld and portable types are usually implemented for temporary monitoring or periodical inspection. Handheld devices are designed to be easily carried by operators and most of them are for limited condition monitoring purposes. Better than handheld condition monitoring types, portable devices include certain types of signal processing analysis and data storage which offers more complete induction motor assessment. In order to gain an understanding about the three types of monitoring devices, Table 2.4 compares their advantages and disadvantages. 
Table 2.4 Advantages and disadvantages types of condition monitoring devices

\begin{tabular}{|c|c|c|}
\hline Types & Advantages & Disadvantages \\
\hline Handheld & $\begin{array}{l}\text { - Easy to be operated but not } \\
\text { necessarily to interpret the result } \\
\text { - Inexpensive } \\
\text { - Fully mobile and flexible }\end{array}$ & $\begin{array}{l}\text { - Limited type of measurement } \\
\text { can be performed } \\
\text { - Only general result } \\
\text { - Need trained operator } \\
\text { - Lack of data storage } \\
\text { - Single channel }\end{array}$ \\
\hline Portable & $\begin{array}{l}\text { - Can collect and record data } \\
\text { - One to two channels } \\
\text { - Performs signal processing based } \\
\text { analysis }\end{array}$ & $\begin{array}{l}\text { - Relatively expensive } \\
\text { - Need trained operator } \\
\text { - Limited data storage }\end{array}$ \\
\hline $\begin{array}{l}\text { Permanent } \\
\text { online }\end{array}$ & $\begin{array}{l}\text { - } \text { Continuous online monitoring } \\
\text { - Provides early detection and alarm } \\
\text { - Sometimes fitted with advance } \\
\text { signal analysis and artificial } \\
\text { intelligent software } \\
\text { - Multiple channels }\end{array}$ & $\begin{array}{l}\text { - Very expensive } \\
\text { - Need expensive maintenance } \\
\text { than other types }\end{array}$ \\
\hline
\end{tabular}

As can be seen from Figure 2.10 and Table 2.4, handheld and portable devices are commonly intended to be implemented in periodical time based maintenance. This handheld or portable device is usually implemented for a small induction machine which is operated occasionally or has a non-critical safety function. However, for a complex and high voltage induction motor, permanent online equipment is needed even though the hardware price and maintenance are expensive. Considering this situation, there is a need of permanent online equipment, which has wide range monitoring capabilities, low-cost, and suitable for operators in industries for small, medium and large induction motors.

Considering low-cost condition monitoring devices, several alternative processing device developments have been carried out recent years. However, several low-cost processing devices are not fully ready to be implemented in practical application as monitoring devices because most of them do not offer an alternative to the commercial units. These low-cost devices are usually based on programmable instrument approaches such as microprocessors, computers, DSPs, PXIs, VXIs and FPGAs as illustrated in Figure 2.11 and discussed in the following paragraphs.. 


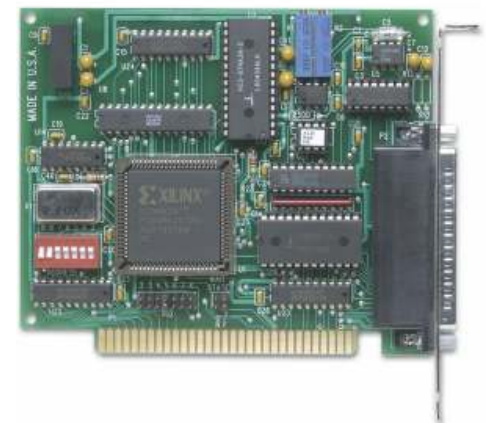

(a)

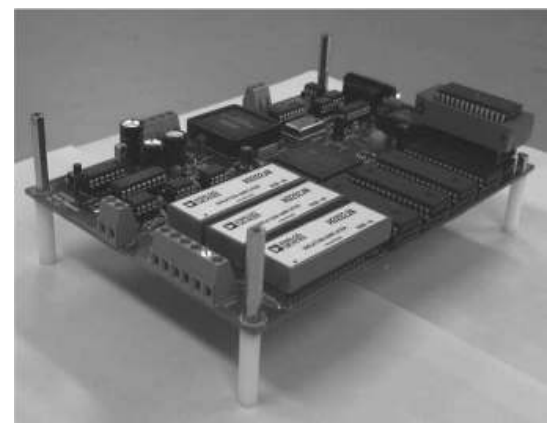

(b)

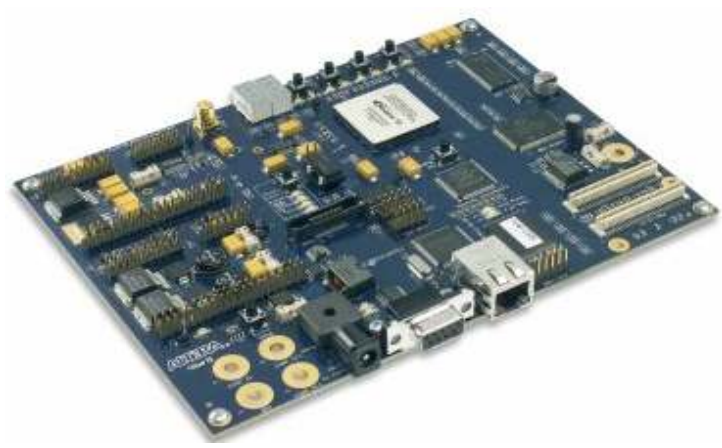

(c)

Figure 2.11 Example of a data processing board for condition monitoring (a) ISA DAS-8 Metrabyte Inc data acquisition card [8], (b) DSP TMS320C32 board [61], (c) Altera NIOS II soft core and a Stratix FPGA board [86]

In a study, Ansari and Baig [8] successfully developed a personal computer (PC) based signal analyzer to monitor the vibration of primary cooling pumps and air compressors of a nuclear research reactor. This device is composed of a $24 \mathrm{MHz}$ PC/AT-386 personal computer, a DAS-8 Metrabyte Inc. 12bit ADC data acquisition card, shown in Figure 2.12.(a), and an FFT analysis software using C programming language. This research successfully outlined the performance of a PC as a condition monitoring device. However, following the extraordinary trend of PC development, plug-in data acquisition cards do not always match with the new generation of PC expansion cards. The ISA expansion card in this research is not available in a new computer. In other words, the plug-in condition monitoring cards depend on PC development. In addition, PCs are not usually designed to be operated in industrial conditions.

In another study, Betta et. al [12] demonstrated a significant improvement in the condition monitoring device. They designed a vibration condition monitoring device based on their previous research hardware [11, 87], a host PC, and a DPC/C40 
board with two concurrent DSPs TMS320C40 and an on-board data acquisition system. The device employs FFT analysis with a simple rule technique for automatic fault detection [12]. This research has demonstrated the capability of DSPs and a PC to diagnose motor failures through the vibration detection technique. However, in this system, the analyzer, which is based on PC memory address in communicating with the DSPs board, needs to be evaluated periodically to ensure the compatibility of the system with current computer development. In addition, a connector of the DPC/C40 board to the PC, which relies on obsolete ISA expansion type, is another serious problem. In other words, further maintenance for this device is becoming impossible.

Another similar device was utilized in diagnosing induction motors, based on the MCSA detection method. In this work, Jung et. al [61] proved the capability of a DSP TMS320C32 board (Figure 2.12.(b)). Unlike the device in [12], the DSP board utilized in [61] is connected to the PC through a serial cable. This serial cable connection is still supported in the latest PC models. Furthermore, in [61] the authors proposed several techniques to guarantee the MSCA diagnosis running properly using FFT analysis. Such techniques include an optimal-slip-estimation algorithm, a propersample-selection algorithm, and a frequency auto search algorithm. However, unlike the device in [12], this system still needs an experienced operator to interpret the signals to predict a fault.

Zhang et.al [86] also enhanced the processing device for condition monitoring by designing a portable vibration analyzer based on FPGA architecture. The authors used Altera NIOS II soft core as a microprocessor controller and a Stratix FPGA, shown in Figure 2.12.(c), a 12 bit 4 channel ADC ADS7841, and a 320 x 240 pixel, 256-Colour LCD. This study pointed out the potential applicability of the FPGA device in condition monitoring. The device developed in [86] was compact and did not require an additional PC for diagnosis or for displaying the results. This device perhaps follows the latest trend of MicroVibe [88], the PDA-based vibration analyzer, as a handheld analyzer. Unfortunately, the authors did not examine the performance of the device using real vibration measurement in electric motors. The work also did not accommodate an appropriate signal-conditioning device. The signal-conditioning is an important issue to deal with the wide range surge voltage output signals and to provide a current excitation mode for certain accelerometer types. Furthermore, the processing device developed in [86] still needs an expert to interpret the fault symptoms. As 
indicated above, most of the processing developed did not fully adapt industrial class hardware to be used in induction motor condition monitoring.

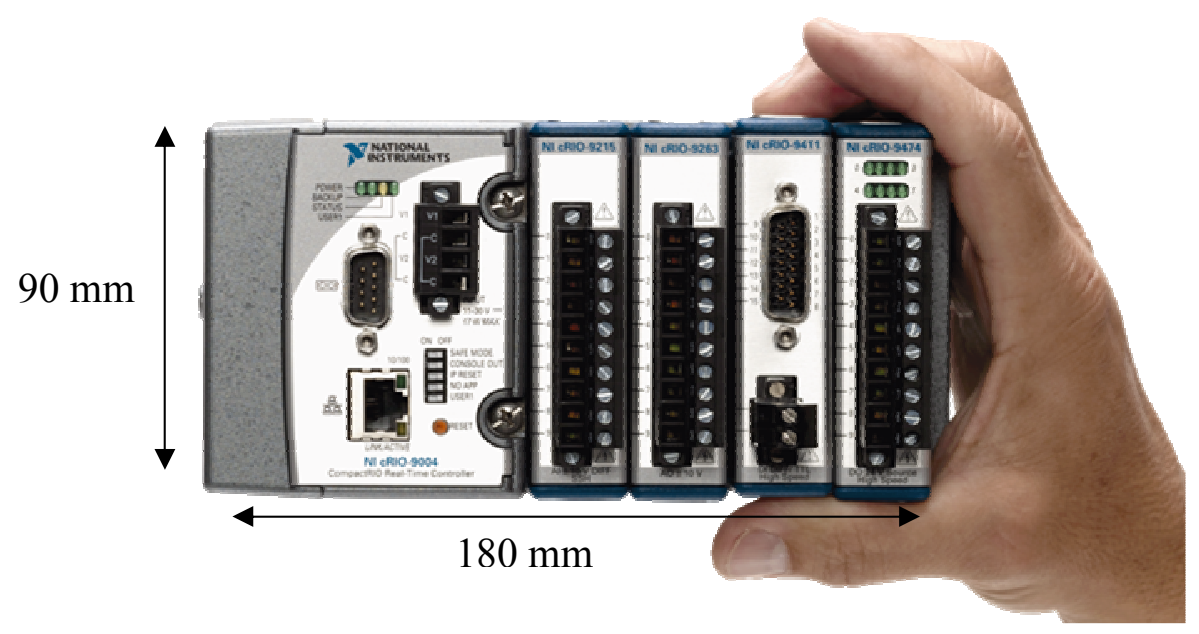

Figure 2.12 A CompactRIO base unit [89]

Recently, due to the development of low-cost compact reconfigurable devices, it has become possible to develop a reconfigurable device to be used in industrial applications, including on-line monitoring of induction motors. One such device is the Compact Reconfigurable Input/Output (CompactRIO ${ }^{\mathrm{TM}}$ ) [90]. The CompactRIO platform, as can be seen in Figure 2.12, has a small rugged, modular, scalable, embedded open architecture system consisting of an FPGA, a real-time processor, and isolated I/O devices [90]. This unique configuration device has industrial class certification (class I and div2) for hazardous locations or potentially explosive, high temperatures, and $50 \mathrm{~g}$ shock capability.

Several industrial applications have utilized the CompactRIO [90]. Dase et. al [91] employed a CompactRIO as an Engine Control Unit in motorcycle prototyping. Al-Naami et.al [92] designed a medical monitoring device to detect Aortic Stenosis Disease based on a CompactRIO device. A CompactRIO was also used in Magnetically Levitated Systems and in a Linear Drives-MAGLEV project as a controller [93]. However, no publications were obtained that utilized the CompactRIO architecture in induction motor condition monitoring, which is the aim of this project.

Compared with other programmable platforms, the CompactRIO can be a real reconfigurable hardware. The CompactRIO offers high-speed, parallel, and real-time processing tasks in one unit. Another advantage is that the development of CompactRIO real-time and the FPGA environment can be accelerated by LabVIEW programming technique. Furthermore, the FPGA is comparable with, or even better 
than, the DSP in term of complex signal processing applications, speed of execution, power consumption and time-to-market development [94]. However, the performance $200 \mathrm{MHz}$ real-time processor of the host controller is less than the performance of the latest PC processor models. Therefore, if the speed is critical, the real-time application running in this type of controller should be optimized carefully. In other words, perhaps this device is a good choice for low to medium level complexity of signal processing applications.

It is clear that there is still a need for an industrial class condition monitoring device, which can be low-cost while requiring little knowledge for operator in understanding the faults of an induction motor. The feasible alternative is to use CompactRIO systems while providing simple indicators about the class of failures or status of the motors. Such devices can be located near the motor, and continuously operated without the need for an experienced operator.

\subsection{Conclusion}

It was concluded from the literature review that an industrial class low-cost condition monitoring device can provide a better solution for numerous induction motors that require continuous monitoring. Such devices may focus on groups of common failures such as bearing, stator, rotor, eccentricities, mechanical set-up and voltage-related problems, through implementation of multiple monitoring techniques. As pointed out in this chapter, the CompactRIO architecture might be the best option for such devices.

The objective of this thesis is to develop an induction motor condition monitoring system based on CompactRIO architecture. Due to the capabilities of this device, selected multiple monitoring techniques as previously discussed in this chapter, can be implemented by applying the FFT data analysis technique. These monitoring techniques are stator current, voltage, vibration and flux leakage monitoring. The implementation of these techniques may give comprehensive results of single or multiple groups of faults. In addition, to help less experienced operators to understand the motor condition, a custom-made CompactRIO indicator module of the motor status will be developed. 
In the next chapter, various fault frequency predictors based on FFT analysis will be discussed. These fault frequencies are classified according to typical common faults in each monitoring technique. 


\section{Chapter 3}

\section{Induction Motors and Fault Frequencies}

\subsection{Introduction}

This study considers a non-invasive monitoring system that can be installed in existing as well as in new motors. The sensor signals utilized in this research include real-time current, voltage, axial flux and vibration signals as they can provide significant and complementary information about the condition of induction motors. As previously mentioned in the Introduction, the signal analysis method utilized in this research is the standard FFT technique, as it can provide valuable and accurate information about a motor's fault frequencies. The fault frequencies can related to the level of severity of faults in an induction motor. For example, the increasing of the peak frequency amplitude could indicate the level of component damage.

In the following sections of this chapter the fault frequencies based on stator current, voltage, vibration and flux leakage monitoring are described. Before that discussion, an overview of the induction motor theory will be presented to ensure understanding of specific components and the relation with generated fault frequencies. The fault frequencies are discussed according to groups of failures. The fault frequency analysis is presented according to studies and formulas carried out by a number of researchers in this field.

\subsection{Brief Overview of Induction Motors}

Induction motors are currently dominating electrical machines in various industries. Simple operation and low maintenance are among the reasons for this. In 
this section, the basic principles, type and operation of induction motors will be covered briefly.

\subsubsection{Overview of Induction Motor Construction}

There are two principal components of induction motors as shown in Figure 3.1: a stationary stator and a rotation rotor. Both of these main parts are placed inside an enclosure, which consists of a frame, and two end brackets (bearing housing). The stator is laid inside the frame. The rotor is fitted inside the stator with a small air gap ranging from $0.4 \mathrm{~mm}$ to $4 \mathrm{~mm}$ with the stator, according to the power of the induction motor. This means that there is no physical contact between the rotor and stator, allowing the rotor to move freely, supported by shaft mounted bearings.

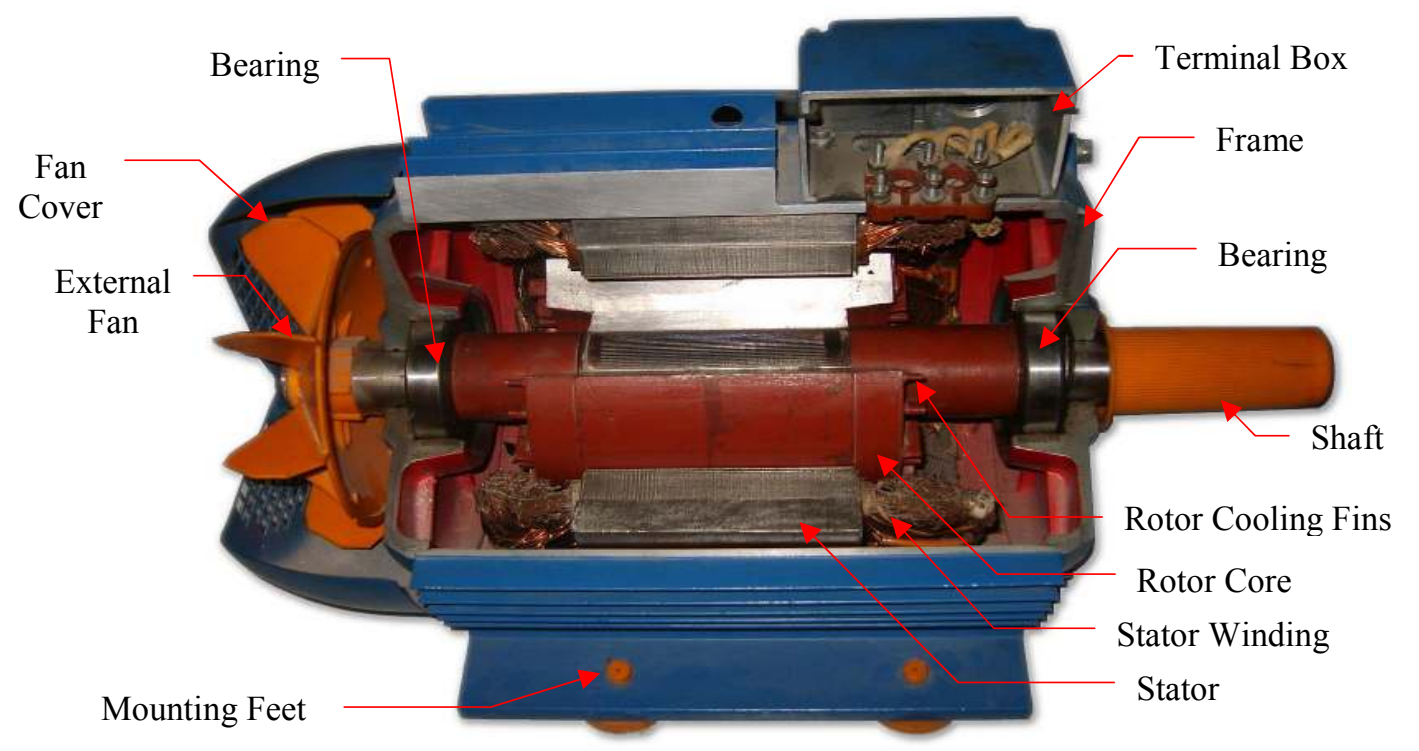

Figure 3.1 Cutaway of squirrel cage induction motor showing main parts [2]

The stator essentially consists of three parts: the stator core, insulation, and coils. As illustrated in Figure 3.2.a, the stator core is made from thin laminations. Several hundred of the laminations are stacked to make a hollow cylinder. Then, as indicated in figure 3.2.b, stator slots lined with insulating material and a coil of the insulated wire are inserted. These stator windings are connected directly to threephase AC supply. 


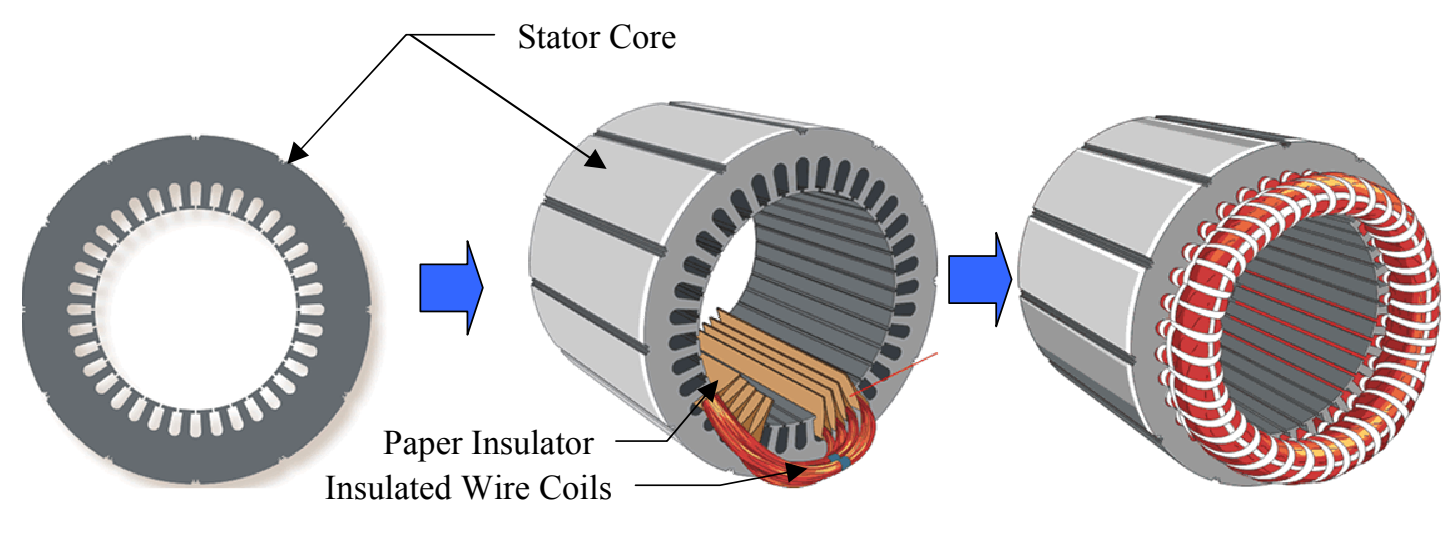

(a)

(b)

(c)

Figure 3.2 Stator construction [95]: (a) laminations,

(b) coil insertions, and (c) a complete stator

The rotor as the second main part of the induction motor determines the type of induction motor. Based on the rotor construction, there are two types of induction motor: the squirrel cage induction motor and the wound-rotor induction motor.

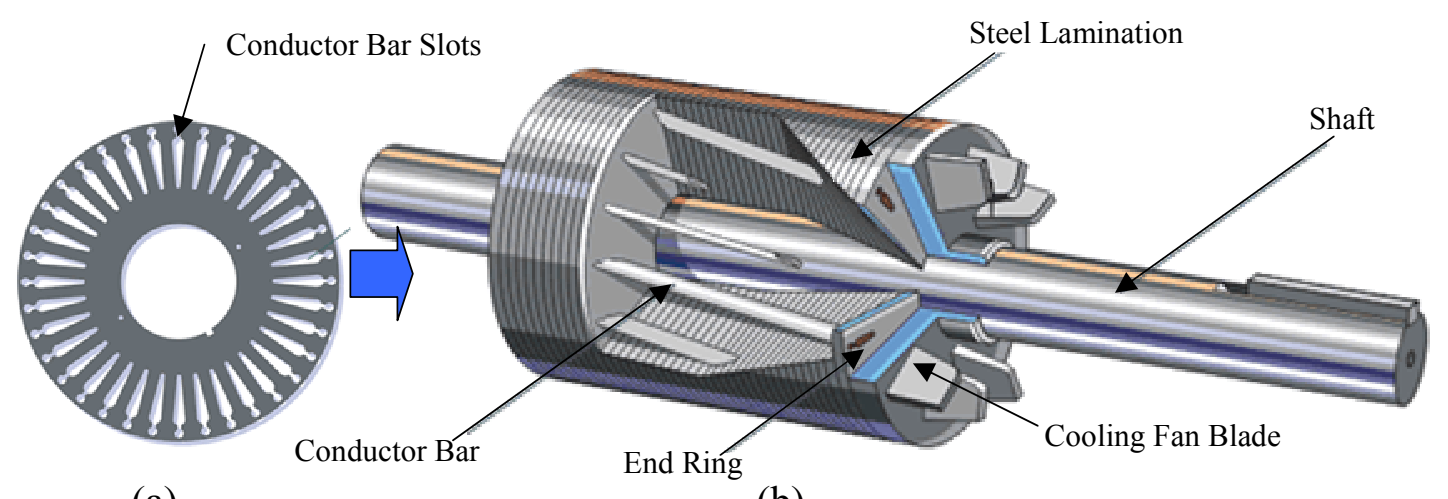

(a)

(b)

Figure 3.3 Cross-section of (a) a squirrel cage rotor, and (b) a complete arrangement of the squirrel cage rotor [95]

As visualized in Figure 3.3, squirrel cage rotor is made from laminated iron cylinders, which are stacked together with slots in its surface to form a rotor core. For the large induction motor, the conductor bars are made from copper in rotor slots connected or short-circuited together by copper or brass end rings. The joint between the bars and end rings is commonly made from electric welding. In some cases, especially for small and medium cage induction rotors, the bars, end ring and cooling fan blades are made of aluminum die-cast in one piece, as the example shown in Figure 3.1. 


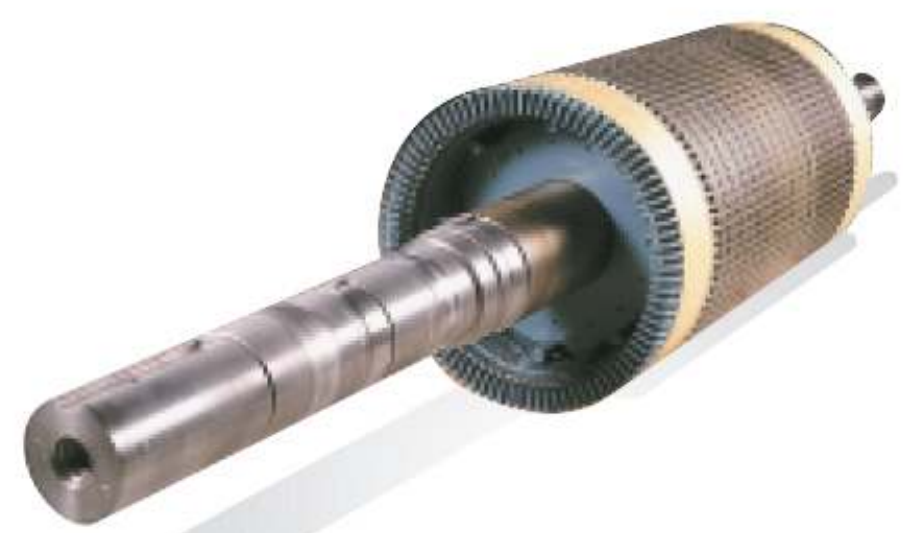

Figure 3.4 Wound-rotor [96]

Unlike the squirrel cage rotor, a wound rotor has three phase windings, which are inserted through the rotor core, similar to the stator. The star connected winding has three terminals which are fastened to slip rings mounted on the shaft, such as in Figure 3.4. Then, carbon brushes mounted on the slip rings are shorted together for normal operation. In many cases, external resistances are inserted into the rotor circuit via brushes to improve starting characteristics. Most wound-rotors are implemented for large induction motors. In this chapter, further discussion is focused on squirrel cage motors.

\subsubsection{Overview of the Principle of Operation}

Basic principle of the induction motor can be understood as the applications of Faraday's Law and Lorentz force on a conductor [97]. As shown in Figure 3.5, a rotating magnetic field is produced by the stator when three-phase power supply is applied to the stator winding. The magnetic field revolves in the air gap between stator and rotor and cuts across the rotor bars. This magnetic field induces a voltage $(E=$ $B l v$ ) according to Faraday's Law in the short-circuited bars of the rotor. Due to the induced voltage, currents will be set up in rotor circuit as shown in Figure 3.6. Indeed, interaction of the rotor currents and stator magnetic field will create a mechanical force or Lorentz force $(F=B I l)$, and hence output torque $(T=F . r)$. From the Lorentz law, this torque will be proportional with the flux density of the stator and the rotor bar current. The rotor will also rotate in the same direction as the rotation of the electromagnetic field in the air gap. In addition, the rotational speed of the magnetic field seen by the rotor is the difference between the speed of the stator magnetic field and the rotor speed. This speed will be explained later in the section. 


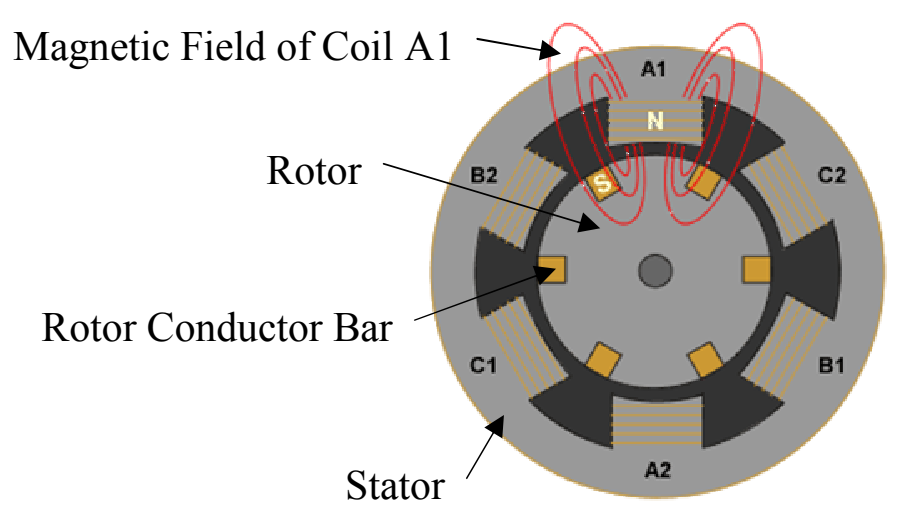

Figure 3.5 The induced voltage rotates the rotor [95]

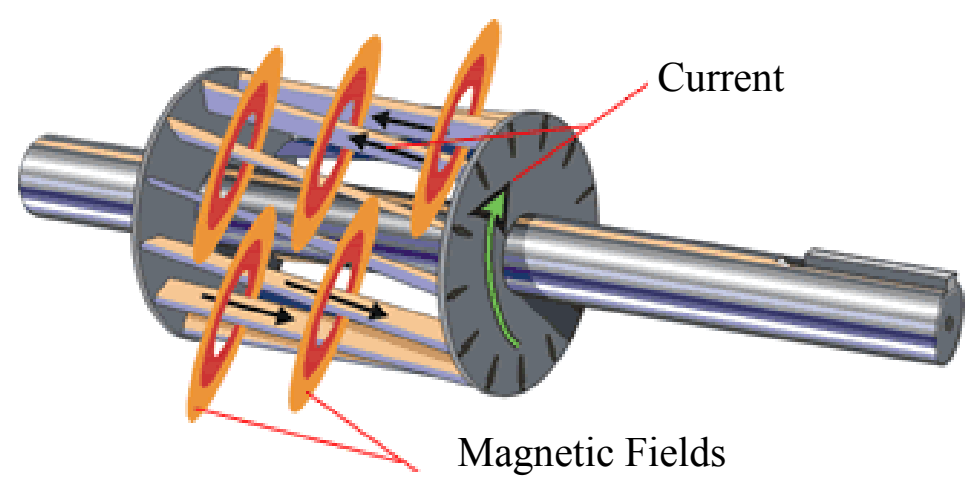

Figure 3.6 Magnetic fields in squirrel cage rotor [95]

\subsubsection{Overview of Synchronous Speed and Slip}

As stated before, an induction motor is connected to three-phase power supply. Illustrated in Figure 3.7.(a), is a stator which has a coil around each of six teeth, supplied by three phase line supplies, phase A, B, and C. Each phase winding consists of a pair of opposite coils connected in serries, passing a current through the phase winding created in the magnetic field along the axis of the coils. The three-phase windings are connected in wye.

Figure 3.7.(b) shows an example of the symmetrical instantaneous value of alternating current $I a, I b$, and $I c$, flowing through the winding of coil A, B and C respectively when the three-phase source is connected. The currents demonstrated in this graph, known as three-phase balanced supply, are shifted in time by an angle of $120^{\circ}$ with equal magnitute in each phase. These currents, in turn, produce a rotating magnetic field in stator dragging rotor described previously. This rotating magnetic field has constant speed synchronised with the frequency of the supply. In other words, as illustrated in Figure 3.7, this speed will depend on frequency of the supply 
and the number of pole pairs. This speed is known as synchronous speed, given by Equation 3.1

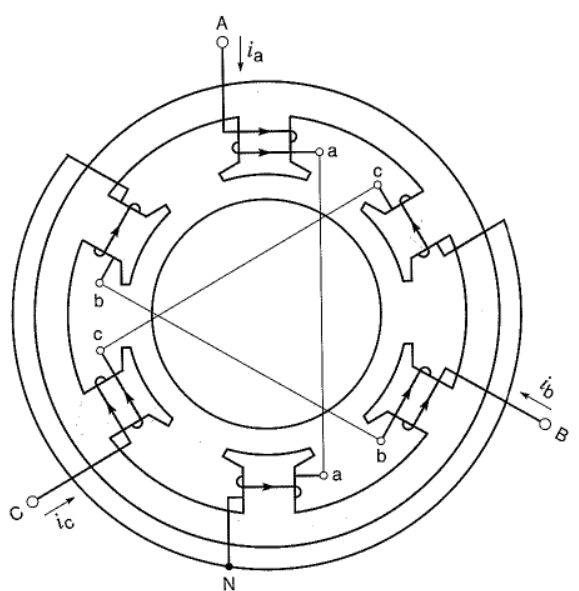

(a)

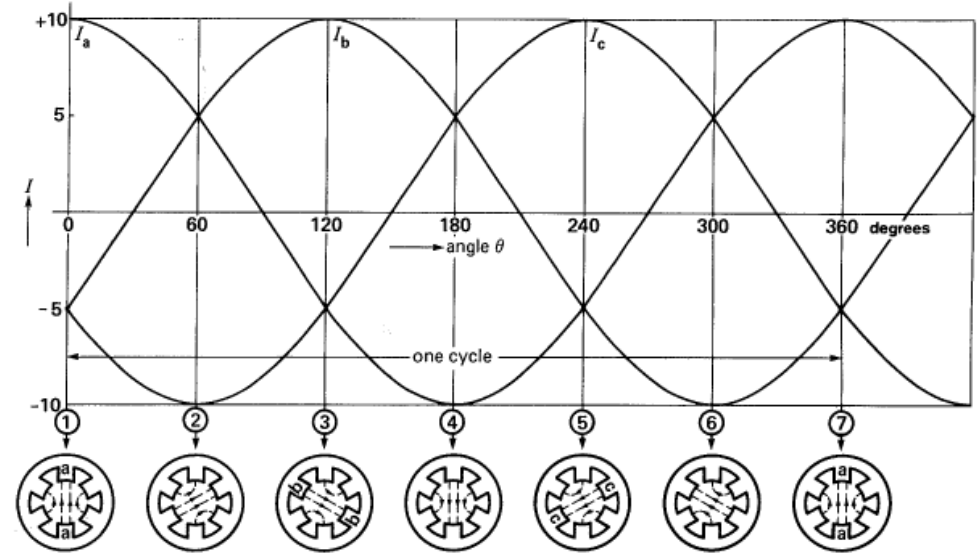

(b)

Figure 3.7 (a) Stator winding arrangement and (b) instantaneous value of currents and magnetic flux distribution produced when 3 phase line supply is applied[97]

$$
N_{S}=\frac{120 f}{P}
$$

Where

$N_{S}=$ synchronous speed of the motor (rpm)

$f \quad=$ frequency of the source $(\mathrm{Hz})$

$P \quad=$ number of poles

The rotating stator magnetic field will accelerate the rotor in the same direction soon after the rotor is released. There will be a relative difference in speed between the stator rotating magnetic speed or the synchronous speed and the rotor. This relative difference in speed is important to enable the magnetic field cutting the rotor, generating induced voltage, as explained in the previous section. In other words, without relative motion between the rotating field and the rotor, there would be no torque produced. Therefore, the rotor speed is always slightly under the synchronous speed of the motor. This speed difference is called slip, which can vary from $0.1 \%$ at no load to about $5 \%$ of synchronous speed at full-load [97] in small machines. The equations 3.2 and 3.3 show how the per-unit slip and slip speed can be calculated. Equation 3.4 can also used to determine the frequency of rotor or shaft. 


$$
\begin{aligned}
& s=\frac{N_{S}-N_{R}}{N_{S}} \\
& \text { slip speed }=N_{S}-N_{R} \\
& F_{R}=N_{R} / 60
\end{aligned}
$$

Where

$s \quad=$ slip

$N_{R}=$ rotor speed (rpm)

$N_{S}=$ synchronous speed of the motor (rpm)

$F_{R}=$ rotor or shaft frequency $(\mathrm{Hz})$

\subsection{Fault Frequencies of Induction Motor}

As stated before, this thesis is focused on groups of faults which are indicated through several studies in induction motor condition monitoring. In this section the most common groups of faults will be discussed, such as bearing, stator, rotor, eccentricity, and set-up related faults. To identify the symptoms of faults, FFT frequency based data analysis according to each sensor types is considered in this section.

\subsubsection{Bearing Faults}

Bearing faults are the most frequent faults in electrical machines. Many studies as discussed in Section 2.3 underlined that this fault contributes to more than $40 \%$ of motor faults. Even though bearings are easy to replace and relatively cheap compared with other parts of an induction motor, undoubtedly bearing faults can cause significant down time and loss of production.

There are many type of bearings which has been developed to be implemented in machines. Commonly, they are divided into four classifications [98]: rollingelement bearing, hydrodynamic bearing, hydrostatic bearing, and electromagnetic bearing. This thesis will cover rolling-element bearings which are commonly installed in small and medium electrical machines [99]. This rolling-element bearing types can be classified into [98] ball bearings and roller bearings. Discussion will be focused on these types of bearing, the cause of failure, and the specific frequencies produced by faults. 


\subsubsection{Ball and Roller Bearings}

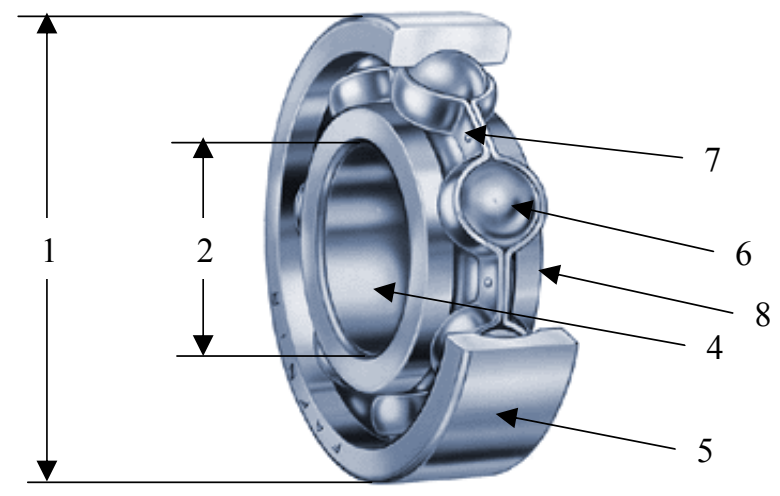

1. Outer Diameter

3. Width

5. Outer Ring

7. Cage

9. Cylindrical Roller

11. Inner Ring Land

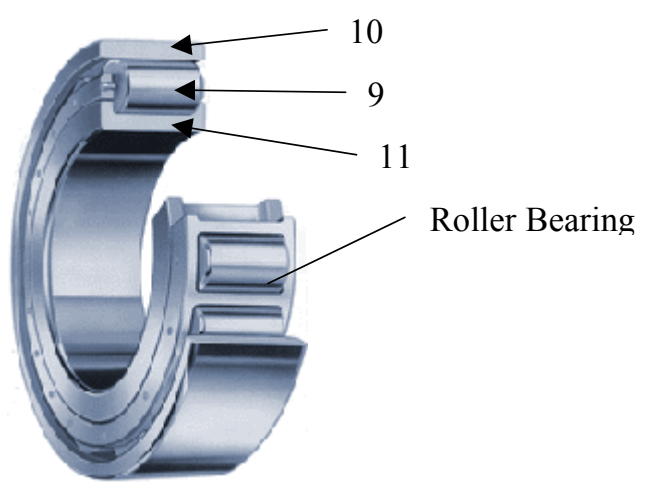

2. Inner Diameter (Bore)

4. Inner Ring

6. Balls

8. Inner Raceway

10. Outer Ring Land

Figure 3.8 Cut-away of ball and roller bearing type shows main parts of bearing

A typical ball bearing and a roller bearing type displayed Figure 3.8, are composed of few main components such as outer ring, inner ring, and balls. The rolling area of the ring is known as a raceway, which can be an inner raceway or outer raceway. Another element, known as a cage, keeps the rolling elements at the same distance and avoids rubbing between them. The inner ring typically will be mounted on the shaft, and the outer ring is fixed inside stationary housing (see Figure 3.1). The ball bearing construction undoubtedly offers simple maintenance and low friction. Most are installed in small and medium motors. The roller type is useful for applications requiring high load carrying capabilities.

\subsubsection{Bearing Failure Causes}

As the most frequent part to fail, bearings have attracted the interest of researchers and bearing manufacturers to find the cause and mechanism of failures, and maintenance. In relation to the causes of bearing failures, many studies have been done since the first bearings were developed.

A study [100] has highlighted that a major contributor to rolling-element bearing problems, e.g. $43 \%$, is poor lubrication. Inappropriate lubrication, either under- or over-, will not allow the bearing to rotate properly on the oil film. This could 
lead to excessive heat, causing the breakdown of the race. This breakdown in turn will increase friction between metal, and result in metal speeding up faults due to microstructure defects.

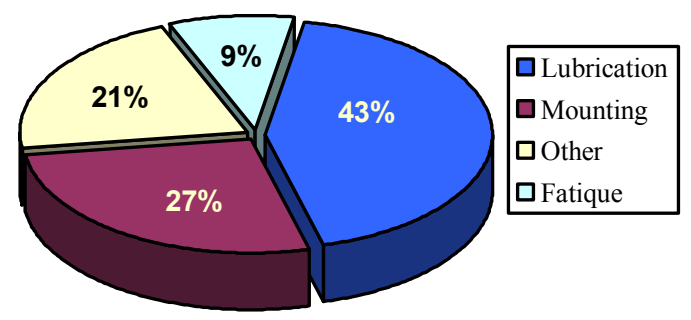

Figure 3.9 Cause of failure in bearing of induction motor [100].

Shown in the figure above, another frequent cause of failure is damage to the bearing during mounting of the bearings to the stationary housings or shaft, around 27 $\%$. This mounting problem causes physical damage, known as brinelling, which leads to premature failures [43]. In the case of brinelling, physical damages are in the form of indentations produced as a result of deformations caused by static overloading [43]. In addition, mounting problems of bearings can occur because of improper installation. Improper installation may lead to several secondary faults such as misalignment, shaft deflections, and cocked/tilted outer or inner races.

Another source of failure, fatigue failure, can occur, even under normal operation, balanced load, and good alignment. Fatigue failures are created because of material imperfections during manufacture. The problem starts as microscopic cracks below the surface of the raceway or rolling elements, which move to the surface. These cracks create detectable vibration, increase noise levels, and induce the further problems: flaking, fluting, spalling, pitting or seizure [46, 101]. Regarding the percentage of other causes of failure illustrated in Figure 3.9, studies [43, 102] have explained other external causes, such as contamination, corrosion, ineffective sealing, improper applications, excessive loading, and the passage of electric current through the bearing.

The most important point in early fault detection is that all defective rollingelement bearings, regardless of the cause and the mechanism of failures, will produce detectable mechanical vibrations at rotational speed of each component. These specific vibration frequencies, known as Ball Pass Frequency Outer race (BPFO), Ball 
Pass Frequency Inner race (BPFI), Train or Cage Frequency (FTF) and Ball Spin Frequency (BSF), occur because of unique constructions and arrangements of the bearing components [16]. These fault frequencies will be explained in the following section.

\subsubsection{Bearing Fault Frequencies}

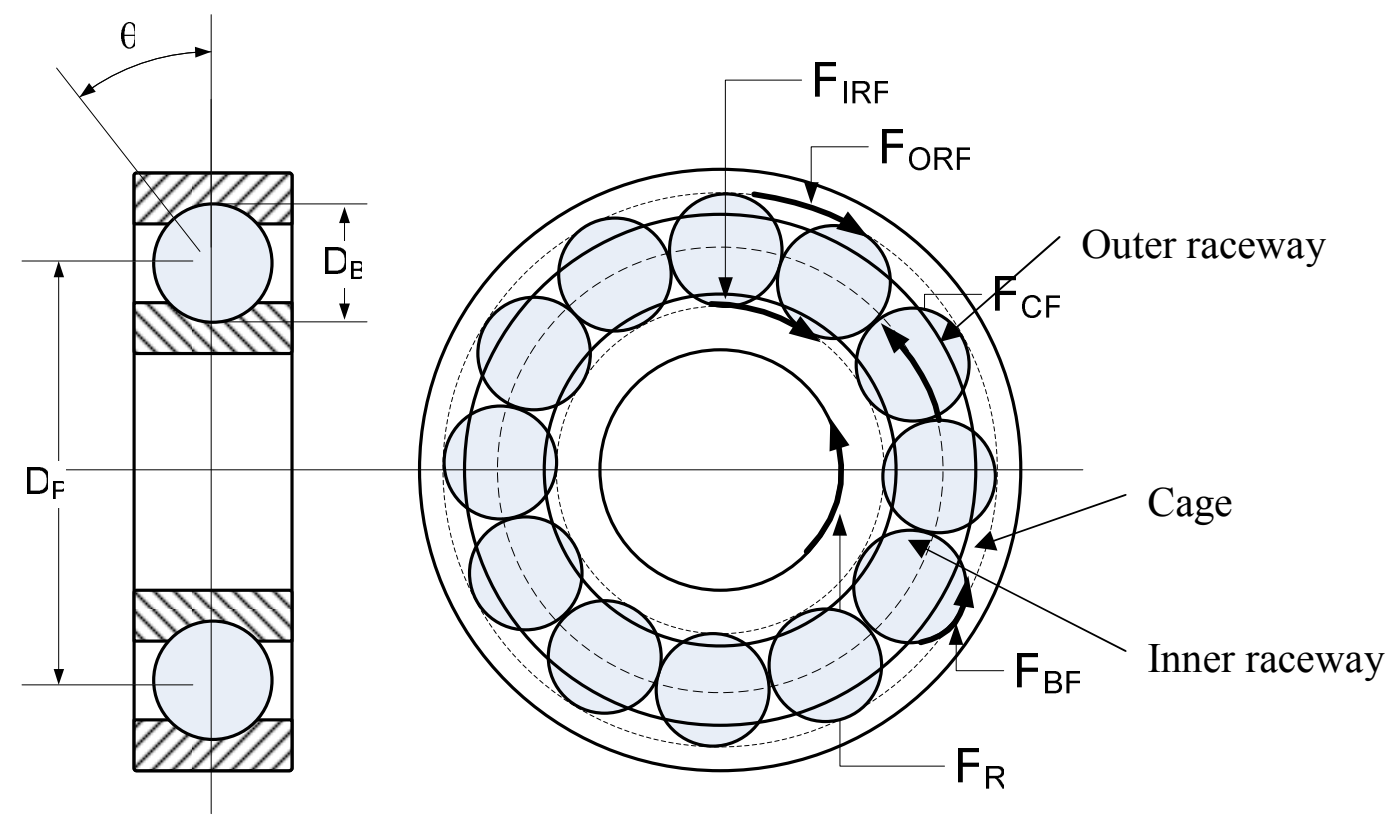

Figure 3.10 Bearing dimension and frequencies of bearing-related faults [100]

One study [4] has pointed out that bearing-related faults might be visible as eccentricity-related faults. Otherwise, bearing faults can be identified in the form of inner race bearing defects, outer race bearing defects, ball defects, and/or cage faults.

These characteristics of rolling-element bearing defects are generated from the specific dimensions of bearings described in Figure 3.10. As illustrated in this figure, a defect in a ball will hit both raceways, inner and outer, during the revolution, causing a specific frequency at twice ball spin frequency. In other words, the ball fault frequency is the rate of each time of the "ringing" of a ball defect rolling over the raceway. This ball defect frequency is given by Equation 3.5 especially for vibration monitoring [41].

$$
F_{B F}=\frac{D_{P}}{2 D_{B}} F_{R}\left(1-\frac{D_{B}^{2} \cos ^{2}(\theta)}{D_{P}^{2}}\right)
$$


Here

$F_{B F}=$ ball fault frequency

$F_{R} \quad=$ rotor or shaft frequency

$D_{B} \quad=$ ball diameter

$D_{P} \quad=$ pitch diameter

$\theta \quad=$ ball contact angle

$s \quad=\operatorname{slip}$

Furthermore, almost similar with ball defect frequency, the vibration of the outer or inner raceway is created when each of the healthy ball bearings rolls over the defects. Unlike ball defects, the vibration produced will appear n-times, according to n-number of balls, during a complete circuit of the raceway. Thus, the equation of inner and outer defects contains multiplication of the number of balls, and fundamental cage fault frequency, such as given by Equation 3.6, 3.7, and 3.8 below.

$$
\begin{aligned}
& F_{C F}=\frac{1}{2} F_{R}\left(1-\frac{D_{B} \cos (\theta)}{D_{p}}\right) \\
& F_{O R F}=\frac{N_{B}}{2} F_{R}\left(1-\frac{D_{B} \cos (\theta)}{D_{p}}\right) \\
& F_{I R F}=\frac{N_{B}}{2} F_{R}\left(1+\frac{D_{B} \cos (\theta)}{D_{p}}\right)
\end{aligned}
$$

Here

$F_{C F} \quad=$ cage fault frequency

$F_{O R F}=$ outer race fault frequency

$F_{I R F}=$ inner race fault frequency

$N_{B} \quad=$ number of balls

It can be seen from the equations above that all require knowledge of bearing parameters. Researchers have proposed alternatives without using these parameters, as indicated in [103], where these equations can be simplified for bearings with 6 to 12 balls (e.g 6200 series) as

$$
\begin{aligned}
& F_{C F}=0.4 * F_{R} \\
& F_{O R F}=0.4 * N_{B} * F_{R} \\
& F_{I R F}=0.6 * N_{B} * F_{R}
\end{aligned}
$$




$$
F_{\text {Bearing }}=\left(0.4 n_{1}+0.6 n_{2}\right) * F_{R}
$$

where, $n_{1}$ and $n_{2}$ are integers $(1,2,3, \ldots)$.

Besides the vibration monitoring capability to detect bearing-related faults, the bearing faults can also be identified using stator current, stator voltage, and flux leakage signals as given in [43]. These monitoring techniques can be utilized based on a simple understanding that unique radial motions between the rotor and the stator will be generated by any bearing defects. As described in the previous section, the bearing defect will produce misalignment, cocked or tilted outer or inner race causing an uneven air gap between the stator and rotor to vary around the airgap. The air gap eccentricity produces predictable frequencies of an electrical supply component containing one of the natural bearing defect frequencies as given in 3.13 [43].

$$
F_{C F}=\left|F_{L} \pm m F_{V}\right|
$$

Where

$F_{L}=$ the supply frequency

$m$ = a positive integer multiplier $(1,2,3, \ldots)$

$F_{V}=$ one of the vibration characteristic frequencies that can be obtained from

Equations 3.5 to 3.8

\subsubsection{Stator Faults}

Many studies have highlighted that stator-related faults are another common problem in induction motors, and can lead to catastrophic failures. The reason for this is that the stator is subject to complex electrical and mechanical stresses, even environmental stresses. One or a combination of stresses might initiate stator faults. These complex stresses, cause of failure including the fault frequency predictors will be discussed briefly in the following sections.

\subsubsection{Stator Failure Causes}

As illustrated in Figure 3.2, the stator consists of two main parts, stator windings and stator core. Based on these main parts, Tavner and Penman in [16] categorized various stator-related faults into stator winding faults and stator core faults. For stator winding faults, Tavner and Penman identified two main locations. The first location is end winding portion faults, which can be in the form of local damage, fretting, contamination, cracking, displacement of insulations, or any turn to 
turn faults, caused by any damage to winding. The second location is slot portion faults such as fretting of insulation, and displacement of conductors. In the case of stator core faults, Tavner and Penman grouped these into lamination faults, such as core hot spot and core slackening, and frame faults, for instance frame vibration, circulating currents, and earth faults.

Thomson and Fenger in [49] explained the biggest concern in relation to the winding faults caused by short circuits which basically can be used to represent other specific faults. There are many types of winding short circuits such as:

1. Turn-to-turn short within a coil

2. Short between coils of the same phase

3. Phase to phase short

4. Phase to earth short

In addition, Thomson and Fenger suggested that pre-warning of failures, which can be in the form of points 3 and 4, could be identified early by continuous evaluation of points 1 and 2 above. In other words, one of the key issues in points of preventing catastrophic winding failures in induction motors is monitoring turn-to-turn faults. These faults mentioned above are commonly caused by a combination of thermal, electrical, mechanical and environmental stresses.

\section{Thermal and Electrical Stress}

Thermal and electrical stresses are contributors to faults, and both may have similar causes. Both stresses could be affected by improper practical operations of induction motors or by the weaknesses of insulation materials. Improper operations include frequent starts, prolonged acceleration time, continuous overloading, and unbalanced incoming line of an induction motor [104].

As an example of improper operation mentioned above, in the case of frequent start within a short time period, or motor jogging operation, the overstress of stator winding insulation can occur because the electric stress is not distributed uniformly. This stress, then, might produce heat spots that are not dissipated properly before another start is taken [22]. This leads to overheating of insulation or thermal stress of the stator winding, which will in turn begin insulation deterioration such as cracking, overheating, tracking and pulverizing [104]. These deteriorations at certain stages cause winding short circuits such as turn to turn, coil-to-coil, phase-to-phase, coil to ground short or iron damage of the stator which will result in stator winding failure. 
In case of unbalanced supply effect, because of much lower negative sequence impedance occurring, much higher current will be induced to the rotor. This over current, even though is really small, might create additional heat which may be present for a considerable time [22]. This additional heat can accumulate and lead to stator winding failure even bearing failures as previously discussed.

\section{Mechanical and Environmental Stress}

Mechanical stress principally can be initiated by the rotor striking to the stator or coil movement [22]. The rotor might hit the stator as a result of several reasons, such as bearing misalignment, shaft deflection, and rotor-to-stator misalignment. This strike can damage the stator core laminations, and stator windings [22]. In addition, this strike will produce a stator core hot spot during operation which could result in damage to the stator winding. In the case of coil movement, during the start-up operation, high stator currents produce a large force on stator coils. This force can then cause stator coil movement which can damage coil insulation and the copper conductor [22].

Environmental stress/contamination is caused by foreign materials, which come into the motor enclosure, such as moisture and dirt. These contaminants will accelerate any electrical, mechanical, and thermal stresses, initiating deterioration of the winding insulation, and stator core defectiveness.

\subsubsection{Stator Fault Frequencies}

An EPRI research as discussed in Section 2.3 has shown that around $73 \%$ of stator faults are insulation problems, either ground insulation or turn insulation. The remaining percentage is caused by frame, core, wedge and bracing faults. Knowledge of the current condition of insulation problems is the most important strategy for preventing immediate catastrophic failure.

Considering interturn stator related faults, in [52], Penman showed that this type of fault causes a change in harmonic distribution of the air gap space. Shorted turn faults can thus be detected based on the time harmonics of the axial flux shown in Equation 3.14 using flux leakage monitoring. Furthermore, Thomson in [49] proved that the Equation 3.14 can also be utilized for stator current monitoring. Based on this equation, Thomson also indicated that a shorted turn could be recognized from any of 
left and right sidebands detected. This stator fault predictor can be observed from the FFT analysis of the current and axial flux signals.

$$
F_{S F}=F_{L}\left(\frac{n(1-s)}{p} \pm k\right)
$$

Here

$$
\begin{aligned}
& n \quad=\text { integers }(1,2,3, \ldots,(2 \mathrm{p}-1)) \\
& k \quad=\text { integers }(k=1,2,3 \ldots) \\
& p \quad=\text { number of pole pairs of the motor }
\end{aligned}
$$

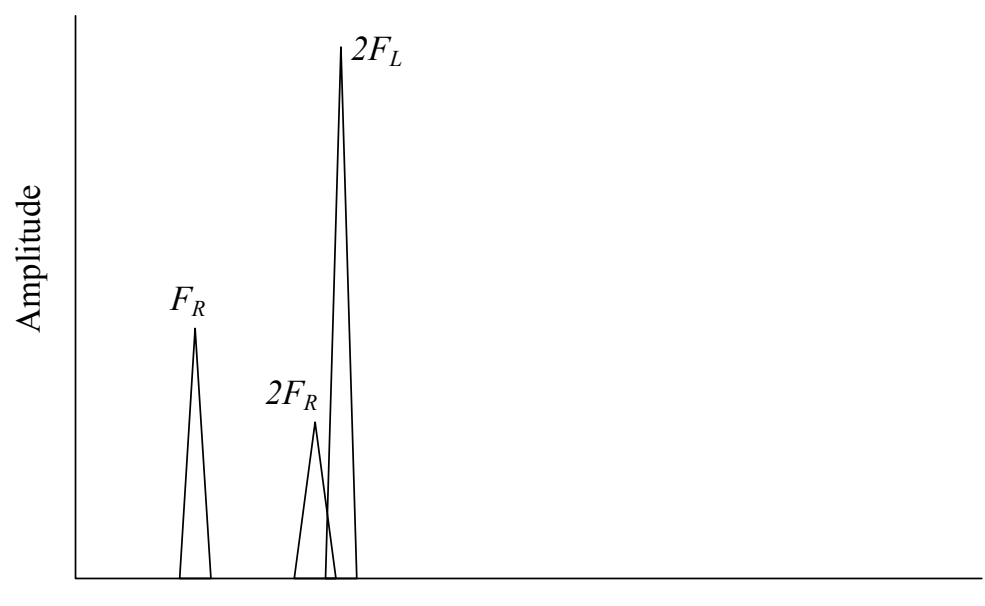

Frequency

\section{Figure 3.11 Stator-related fault frequencies of vibration monitoring}

For the vibration monitoring technique, stator-related faults can be identified of high amplitude of $2 F_{L}$. In [17], stator-related fault frequencies are indicated by $2 F_{L}$ or a combination of $F_{R}, 2 F_{R}$ and $2 F_{L}$ illustrated in Figure 3.11. These fault frequencies can be indications of shorted laminations, stator loose laminations, or stator eccentricities.

\subsubsection{Rotor Faults}

Even though rotor-related faults are not the highest contributors to induction motor failures, rotor failures are potential problems for industries and might an initiate other damages. As has been noted, rotors are divided into squirrel cage and wound rotor types. Since the focus of this thesis is on the squirrel cage type of induction motors, in this section the discussion of rotor-related faults, causes of failures and fault frequency predictors will be carried out regarding this cage type. 


\subsubsection{Rotor Failure Causes}

Squirrel cage rotors are designed basically following four types: aluminum die cast, copper die cast (CuDC), fabricated aluminum bars (AlBar) and fabricated copper bars (CuBar) [105]. Currently, due to cost of construction, and an advance in casting technology, cast rotors are designed not only for small induction motors but also for motors around $3000 \mathrm{~kW}$ [106]. Fabricated copper bar rotors are still popular especially for motors under multiple restart applications.

As indicated in [16], major rotor faults can be divided into rotor winding related problems and rotor body (rotor shaft) defects. It has been pointed out that rotor winding problems can be in the form of cracking in the rotor bars or at the cage end rings. Furthermore, it has also been mentioned that the cracking could also occur in the rotor body. In [4], Thomson summarized several experiences demonstrating that broken rotor bar and end ring faults can become a serious problem in cage rotors [49]. Both die cast and fabricated rotors may suffer from this type of fault. Especially for the die cast squirrel cage rotor, it is almost impossible to repair [105]. Fabricated rotors however are repairable [105], but this rotor type is expensive compared with the cast rotors. In other words, rotor bar and end ring failure analysis is demanded in squirrel cage rotor applications.

The failures of broken rotor bars and end ring faults have many causes. For example, broken rotor or end ring faults may be initiated by imperfect manufacture of casting for die cast rotors $[4,74]$, or poor jointing in the case of brazed or welded end rings. These defects, consequently, could lead to an increase in temperature due to high resistance. Thus, overheating happens and initiates the cracking of rotor bars. The cracks eventually occur in cage rotors and also in the end rings where the bars are not supported by the rotor core. The same defects may also occur because of motor speed fluctuations or repeated starts [4, 74]. Repeated starts causing high currents generate a large force on bars. Repeated large forces produced from these events then may cause material fatigue, which may initiate cracks at the joint between bars and ring. Similarly with stator fault cases, the rotor broken bar might also be initiated by voltage unbalance, single phasing, contamination or corrosion, and vibration.

Besides causing problems in rotor bars, the stress mentioned above may also initiate problems in the rotor shaft. Even though the percentage of rotor shaft faults is relatively small, integrity failures of rotor shaft may occur because of cracks caused 
by defective rotor forging during the fabrication. Similarly with the rotor winding problems, thermal cycling due to excessive starting or negative sequence may also take place, resulting in cracking.

\subsubsection{Rotor Fault Frequencies}

To detect broken rotor bars and end rings, several approaches have been proposed. Most rely basically on interaction of the rotor and the stator rotating field. As discussed in Sections 3.1.2 and 3.1.3, normally there is only a forward rotating field of the rotor, because of the symmetrical nature of the stator-rotating field. If one of the rotor bars is broken, there will be an asymmetrical magnetic field causing a resultant backward rotating field at slip frequency of the rotor. This will induce electro magnetic field and current in the stator winding at specific fault frequencies.

These fault frequencies of broken rotor bars can be determined from the lower sideband equations in Equation 3.15 [49], while the upper sideband is due to rotor speed oscillation. In Equation 3.16 [107], the sequence of sidebands may also give an indication of broken rotor bars.

$$
\begin{aligned}
& F_{B R B}=(1 \pm 2 s) F_{L} \\
& F_{B R B}=(1 \pm 2 k s) F_{L}
\end{aligned}
$$

where $\mathrm{k}$ is integers $(k=1,2,3 \ldots)$ and $F_{B R B}$ is the detectable broken rotor bar frequencies. It should be noted when employing these equations that the load, the load inertia, and the machine speed affect the magnitude of these side bands [108].

$$
F_{B R B}=\left[\left(\frac{k}{p}\right)(1-s) \pm s\right] F_{L}
$$

Here $\mathrm{k}$ is harmonic index $(\mathrm{k}=1,2,3 \ldots)$ and $p$ is the number of pole pairs of the motor.

The Equation 3.17 can be utilized during the spectral component analysis of current, voltage and flux signals for broken rotor bar (BRB) fault detection. In [56], this approach is believed to offer an alternative to an ambiguous decision which may exist through previous approaches using Equation 3.15 and 3.16. At low frequency spectra or harmonics of fault frequencies of a broken rotor bar may exist at the same frequency with rotor eccentricity, variation of cage conductivity, bearing misalignment, and magnetic orientation of the laminations [56]. Therefore, high harmonics might confirm the presence of rotor bar faults at low harmonics. Another 
technique is to compare the rotor bar fault frequency amplitudes between stator voltage and stator current, and subtract the magnitudes to see if the stator current component is due to the supply voltage.

It has also been reported in [7] that the fault frequency components of BRB are observable in the vibration signal spectrum as increasing amplitudes of the sidebands of the pole pass frequency near $F_{R}$ and its odd harmonics as given in Figure 3.12 (a) and 3.12 (b). These fault frequencies may also be a symptom of bad joints between the rotor bars and end rings, or shorted rotor laminations. The pole passing frequency, $F_{P}$ is given by

$$
F_{P}=\left(F_{S} * p\right)
$$

where $F_{S}$ is the slip frequency, which is equal to the difference between synchronous speed and $F_{R}$.

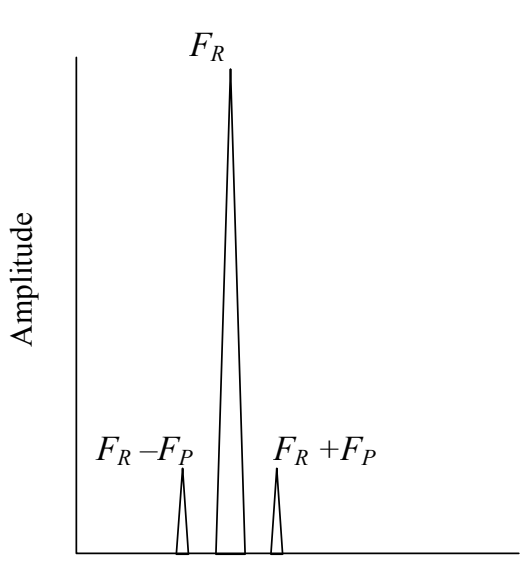

Frequency

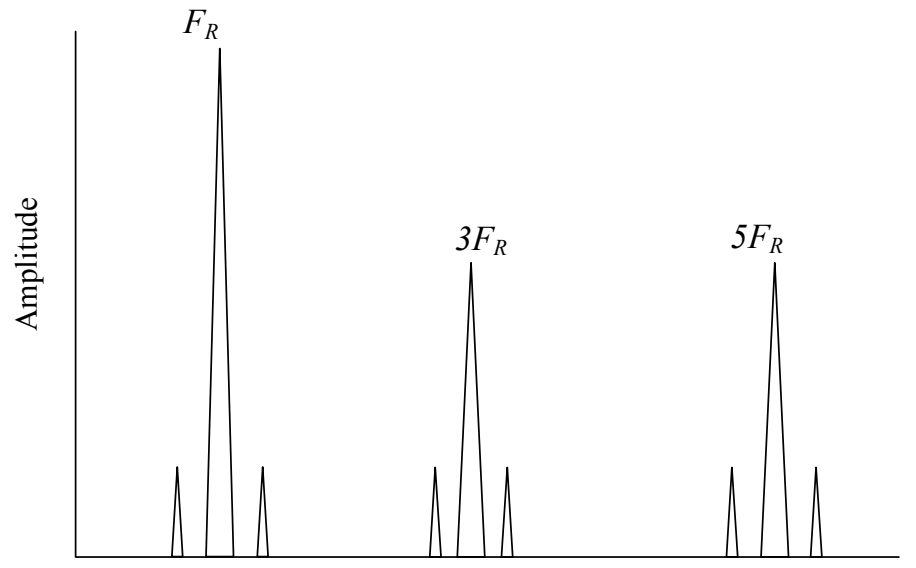

Frequency

Figure 3.12 Broken rotor bar, bad joint and shorted lamination fault frequencies of vibration monitoring

Furthermore, in the case of loose rotor bars, the fault frequency predictor can be defined by

$$
F_{\text {RBPF }}=\left(R * F_{R}\right)
$$

where $R$ is the number of rotor bars in the motor [7]. The sidebands of $2 F_{L}$ around the first harmonics of $F_{R B P F}$ and the second harmonics of $F_{R B P F}$ are also good indicators for this fault, as shown in Figure 3.13. It can be seen in this figure that the second harmonics of $F_{R B P F}$ are higher than the first. 


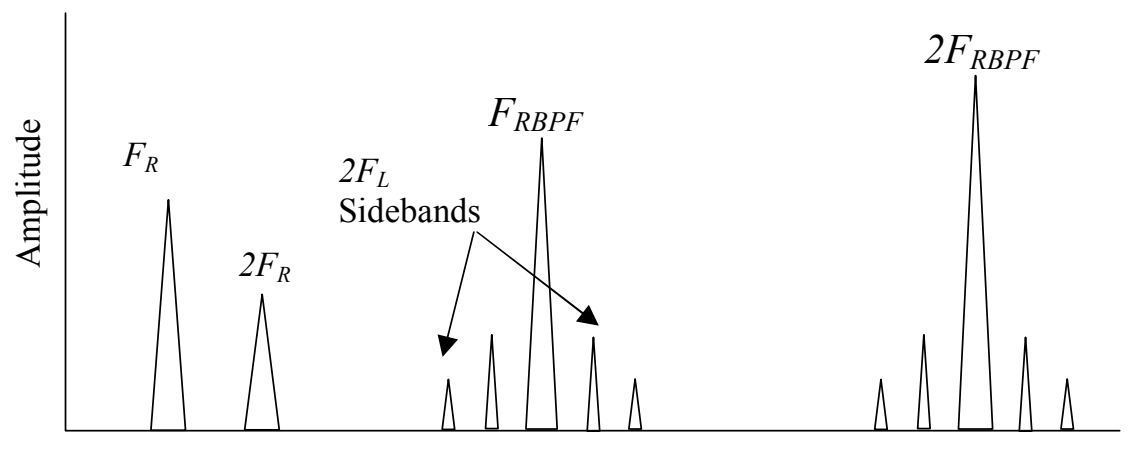

Frequency

Figure 3.13 Loose rotor bar fault frequencies of vibration monitoring.

\subsubsection{Eccentricity Faults}

Eccentricity-related faults occur when the rotor is not positioned in the magnetic centre of the stator in a radial or axial direction [28]. Commonly, an air-gap of eccentricity is allowed in electrical machines of up to $5 \%$, or $10 \%$ in a few cases. However, this air-gap eccentricity between rotor and stator needs to be kept as small as possible to reduce vibration noise, and minimize unbalanced magnetic pull [4]. If the air-gap eccentricity is too high, at the certain level of eccentricity the unbalanced radial forces can drive the rotor to stator rub damaging the rotor, the stator core and the windings [19]. In the case of induction motors, because the air-gap is smaller than in other types of electrical machines, they are more sensitive to the changes of air gap.

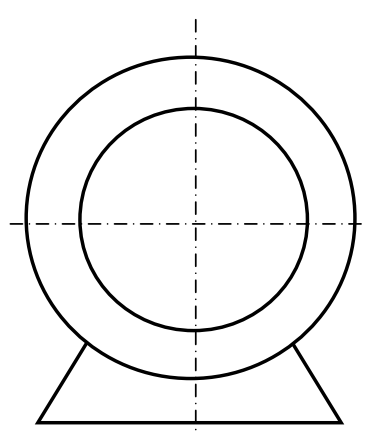

a) concentric

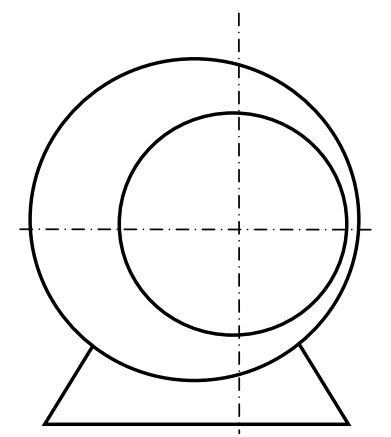

b) static eccentricity

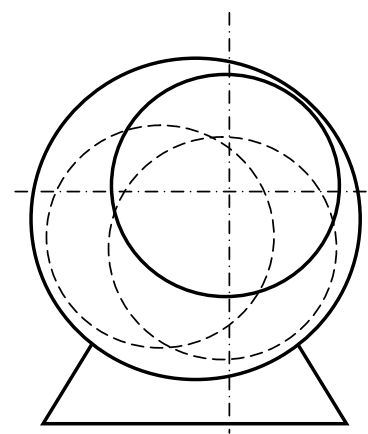

c) dynamic eccentricity

Figure 3.14 Types of Eccentricity

Normally, rotor and stator have the same centre, as shown in Figure 3.13.(a). However, in case of eccentricity there will be two possible positions, as shown in Figure 3.13.(b) and 3.13.(c). These two types of eccentricity are static air-gap eccentricity and dynamic air-gap eccentricity. The static eccentricity means that the minimum air-gap length position is fixed in space. In the dynamic eccentricity, the 
position of the minimum air-gap rotates with the rotor. In other words, the centre of the rotor is not at the centre of rotation. These eccentricities commonly occur at the same time.

\subsubsection{Eccentricity Failure Causes}

Eccentricity faults are commonly caused by imperfect manufacture, installation and other mechanical defects. For example, in the case of static eccentricities, ovality of the stator core or incorrect positioning of the rotor or stator at the commissioning stage may cause this eccentricity problem [57]. In case of dynamic eccentricity, it can be the result of bearing wear or misalignment, which interestingly, in turn, may increase the force acting on the bearing, stimulating further bearing failures [4]. In addition, dynamic eccentricity may also be initiated by incorrect manufacture of the components, a bent rotor shaft, or mechanical resonance at the critical speed [57].

\subsubsection{Eccentricity Fault Frequencies}

Eccentricity components can be identified from variation of air gap flux density. Variations of air gap flux density will affect induced stator current, producing many harmonic frequencies. As given in [19] by using MMF and permeance approach, the Equation 3.20 below, which is basically the function of rotor slotting, and static and dynamic eccentricities, can be implemented as the predictor through analysis of current, voltage and flux signals.

$$
F_{S L O T+E C C}=F_{L}\left[\left(k R \pm n_{e c c}\right) \frac{(1-s)}{p} \pm n_{w s}\right]
$$

Where

$\mathrm{k}=$ harmonic index $(\mathrm{k}=1,2,3, \ldots)$

$p=$ number of pole pairs of the motor

$n_{w s}=$ supply frequency harmonic order $\left(n_{w s}=1,3,5,7, \ldots\right)$

$n_{e c c}=$ eccentricity order number that is " 0 " for static eccentricity, " 1 " for dynamic eccentricity

$R=$ number of rotor slots 
However, in [109], the authors pointed out that not all combinations of number of rotor slots $(R)$ and pole pairs $(p)$ can be implemented in Equation 3.20 to obtain the real harmonic frequencies. This is because additional static or dynamic harmonic frequencies will be introduced at certain $R$ and $p$ combinations. The authors proposed a relationship of $R$ and $p$ that may produce detectable frequencies of static or dynamic eccentricity.

$$
R=2 p[3(m \pm q) \pm r] \pm k
$$

where $m \pm q=0,1,2,3 \ldots, r=0$ or $1, k=1$ or 2 .

In the presence of both types of eccentricity (static and dynamic), the lower sideband of fault frequencies near fundamental frequency can be given by Equation 3.21 regardless of the number of rotor slots $[56,64]$.

$$
F_{E C C}=F_{L}\left[1 \pm k \frac{(1-s)}{p}\right]
$$

where $k=1,2,3 \ldots$

Similar to Equation 3.20, the authors in [57] also concluded that Equation 3.22 can provide indication of significant detectable frequencies at certain $R$ and $p$ combination with $k=1$.

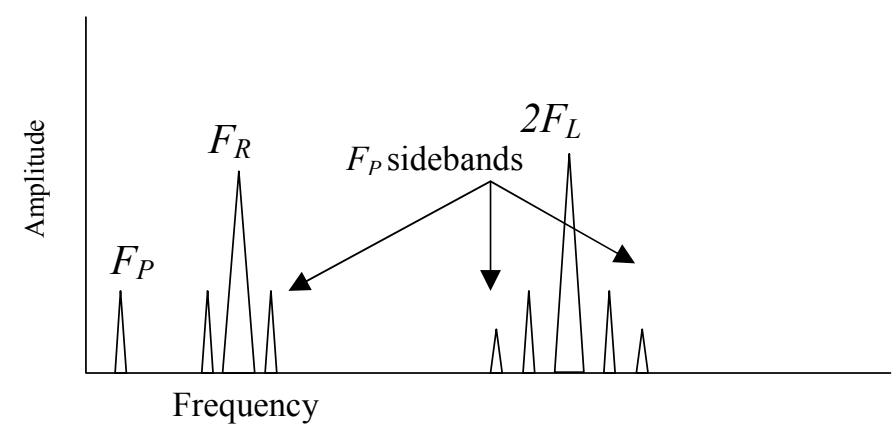

\section{Figure 3.15 Eccentricity fault frequencies of vibration monitoring}

In case of vibration signal analysis, the predictors of fault frequencies are developed based on interaction of the rotor and stator, where the force will vary twice during a single current cycle [7]. The fault frequencies in this reference were defined by pole pass frequency $\left(F_{p}\right)$ sideband components around $2 \times F_{L}$ and $F_{R}$ as illustrated in Figure 3.15. It should be noted that the pole pass frequency in the real machines appears at low frequency range (below $10 \mathrm{~Hz}$ ). In addition, a beat frequency may occur between the frequencies, $2 F_{L}$ and the closest running speed harmonic $k F_{R}$. 


\subsubsection{Other Faults}

Other possible faults that can occur in induction motors are mechanical set-up related faults. Among mechanical set-up related faults, induction motors commonly suffer from unbalanced mechanical faults, misalignment, and looseness. Such faults are recognized mostly by vibration analysis.

Another fault, which commonly exists in induction motors is voltage-related fault. This fault may present in various forms, such as unbalanced line voltage, ground faults [54] or loose connectors (phasing problems) [7]. These voltage faults are also known as hidden faults, because the impact of hidden faults does not directly cause an immediate loss during the operation of the induction motor.

In this section, general information concerning these common mechanical setup related faults, and voltage-related faults including fault frequency predictors, will be discussed.

\subsubsection{Mechanical Unbalance}

Many experiences indicate that unbalance is the most common mechanical fault in induction motors. In [7], mechanical unbalance is defined as uneven distribution of mass about a rotor's rotating centreline. In other words, the mechanical unbalance of a rotor occurs if the rotating centreline of the rotor, known as principal inertia axis, does not coincide with the geometric centreline, as illustrated in Figure 3.15 .

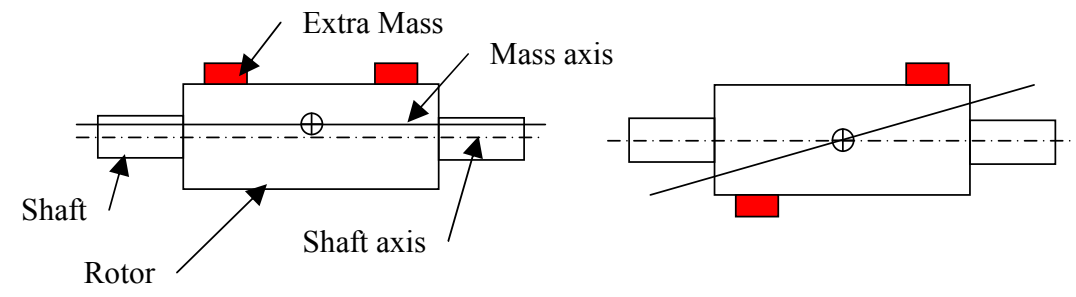

a) static

b) couple

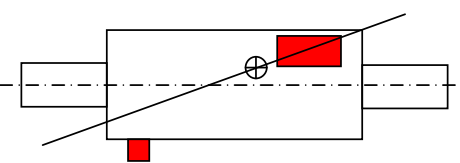

c) dynamic

Figure 3.16 Types of mechanical unbalance

Figure 3.16 shows three types of unbalance that may occur in induction motors. Static and couple unbalances are determined from the position of the principal inertia axis, either parallel or exactly $180^{\circ}$ apart in two planes perpendicular to the axis of rotation [110]. In the case of dynamic unbalance, static unbalance and couple unbalance can occur at the same time, as illustrated in Figure 3.16.(c). All can be 
caused by imperfect fabrications such as voids and porosities, by manufacturing tolerances, or by centrifugal forces during the operation, causing a slightly bow shaft [110]. Furthermore, any of these mechanical unbalance faults are indicated from the high amplitude of $1 F_{R}$ in a radial direction, shown in Figure 3.17 [7] using vibration monitoring. According to Finley et al in [54], the unbalance of the external fan may also result in similar fault frequency both in radial and axial directions on the nondrive-end side.

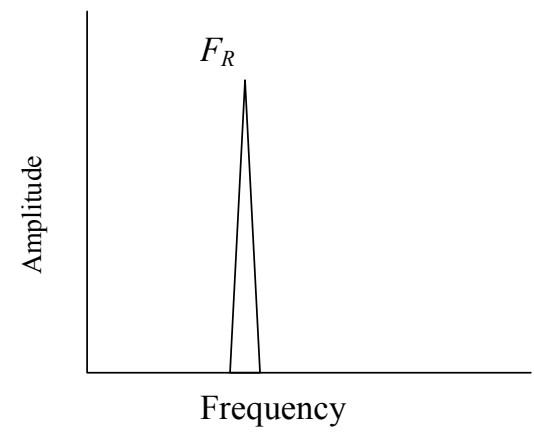

Figure 3.17 Fault frequency of mechanical unbalance.

\subsubsection{Misalignment}

Misalignment may occur in many parts of a motor, for example in the shaft between two bearings, in the coupling of two machines, and in meshing gears. In this thesis, the discussion of misalignment is focused on the first and second types.

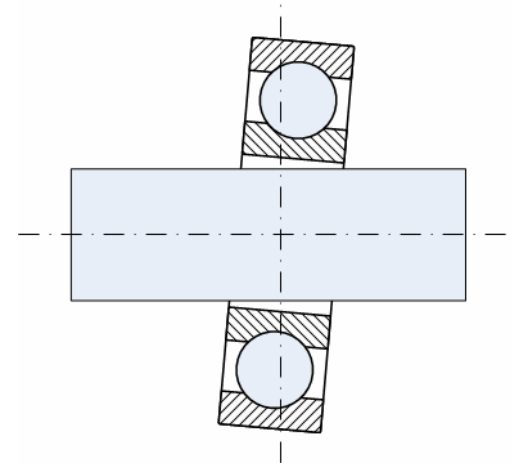

Figure 3.18 Misaligned bearing

For the first misalignment type, as previously discussed in regard to the bearing faults, the misalignment occurs because the bearings are not accurately lined up with the shaft [7] as shown in Figure 3.18. This will produce considerable vibration in axial direction, which is indicated by $1 F_{R}, 2 F_{R}$, and $3 F_{R}[7]$. For the second type, the coupling misalignment can be in the form of parallel misalignment or angular misalignment. In the case of angular misalignment, the axes of two shafts meet at a specific angle. In the case of parallel misalignment, the axes of two shafts are parallel 
and have an offset. These misalignments produce vibration fault frequencies [7] as shown in Figure 3.19.(a) and 3.19.(b) below. As can be seen in this figure that high $F_{R}$ indicates angular misalignment and parallel misalignment is indicated by high amplitude of $2 F_{R}$ than other frequencies.

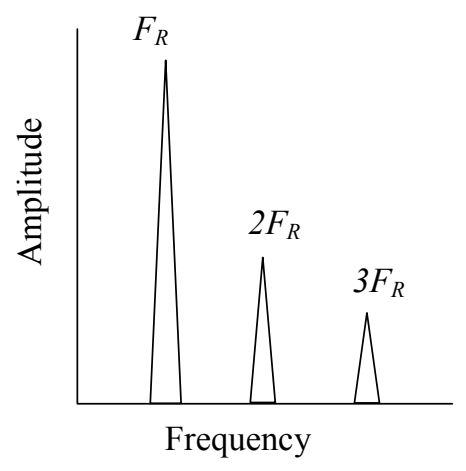

a) angular or bearing misalignment

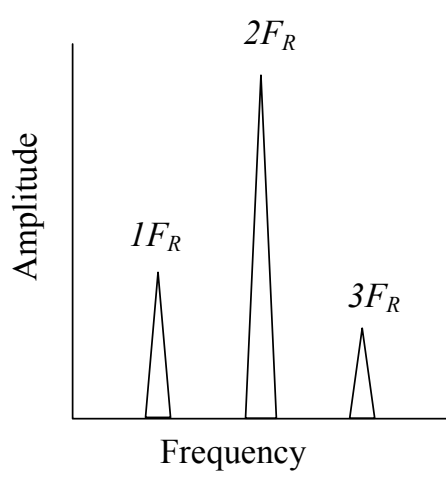

b) parallel misalignment

\section{Figure 3.19 Misalignment fault frequencies using vibration monitoring}

\subsubsection{Looseness}

Similar with misalignment, looseness may occur in several parts of an induction motor. Looseness can be divided into internal assembly looseness, looseness between machine and base plate, and structural looseness [7].

The internal assembly looseness occurs inside the induction motors, such as a bearing in its cap, and a rolling element bearing on a shaft [7]. Improper fit between these parts may cause this looseness. This looseness will lead to non-linear response of the loose part to the exciting force of the rotor, which creates rich harmonics due to truncations of time waveform as shown in Figure 3.20.(b).

If there is looseness of mounting-feet bolts or cracks in the frame or bearing pedestal, the second category of looseness occurs [7]. This looseness may initiate a rocking motion of the mounting feet, producing harmonics of rotor rotations, illustrated in Figure 3.20.(c).

Unlike previous types of looseness, structural looseness, known as soft foot, will generate only $1 F_{R}$, as indicated in Figure 3.20 [7]. This structural looseness produces fault frequency symptoms similar to the mechanical unbalance. Different to the looseness between motor and base plate, this type of looseness is caused mainly by the lack of stiffness of the base plate of the motor, the motor's mounting feet, or foundation. 


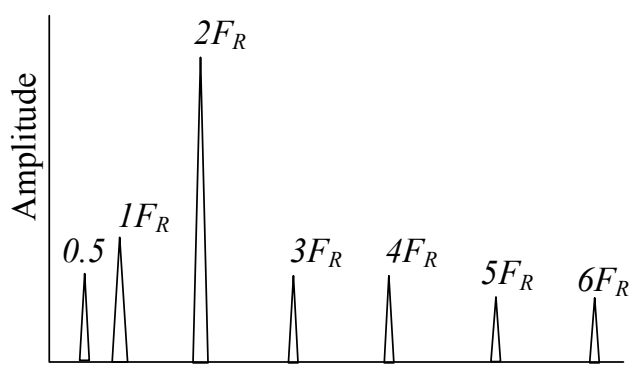

Frequency

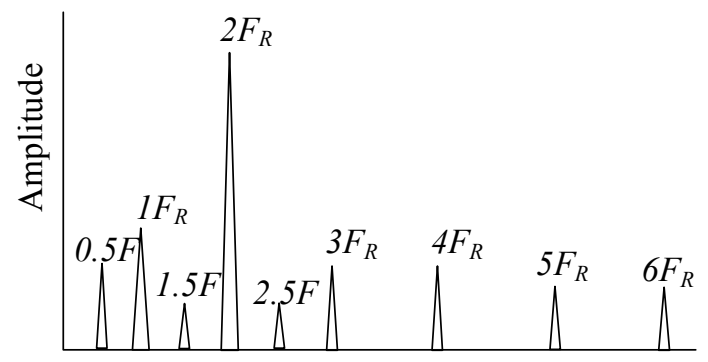

Frequency

a) internal assembly looseness

b) looseness between motor and base plate

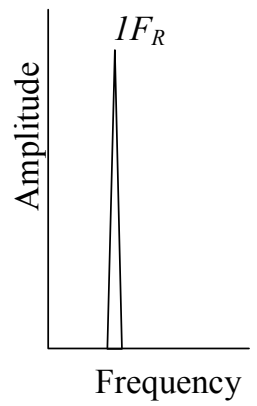

a) structure looseness

Figure 3.20 Three types of fault frequencies of looseness using vibration monitoring

\subsubsection{Voltage problems}

As stated previously that many problems are a result of power supply variations. These power supply variations can be in the form of under voltage supply, over voltage, unbalanced line voltage, ground faults, and phase problems [102]. The result, for example as indicated in Section 3.3.2.1, is that stator winding faults can be initiated by unbalanced voltage or over voltage due to overheating of the winding. Because of these facts it is clear that detecting the cause as early as possible is beneficial in order to prevent catastrophic failures.

A study in [54] indicates that voltage problems mostly can be determined through the presence of a high $2 F_{L}$ component using vibration monitoring. This frequency represents interphase faults, ground faults and unbalanced line voltage. Especially for interphase and ground faults, the $2 F_{L}$ is also followed by high magnitude of $l F_{L}$. Both frequencies can be determined from radial direction of vibration measurement. 


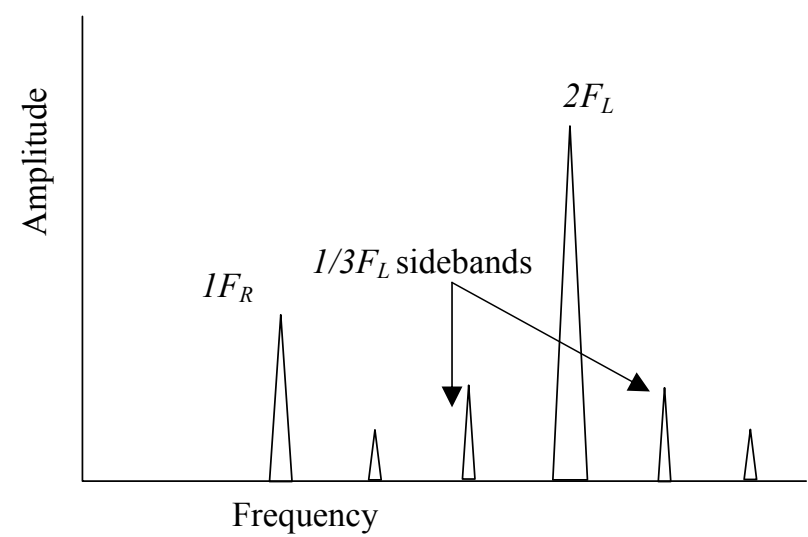

Figure 3.21 Supply phasing problem fault frequencies

In [7], supply phasing problems can be identified through frequencies shown in Figure 3.21 through vibration monitoring. As shown here $1 / 3 F_{L}$ sideband frequencies surround $2 F_{L}$, and this frequency level the vibration level may be higher than $25 \mathrm{~mm} / \mathrm{s}$ [7]. These fault frequencies appear especially when a defective supply power connector in the motor supply is making contact at irregular intervals.

\subsection{Sensor Parameters}

Based on various faults, there is a need to determine sensor parameters for appropriate fault frequency detection. As pointed out in Section 2.3, there are various techniques and sensors of the condition monitoring. In this section, these techniques and sensors are combined with fault frequencies previously discussed to reveal sensor parameters and an arrangement that may be suitable for maximum results of signal processing feature extraction. These sensors, parameters and position are summarized in Table 3.1 and illustrated in Figure 3.22.

Table 3.1 indicates signals and the bandwidth parameter for the measurements. It is common that condition monitoring aims to detect as many harmonic frequencies as possible. The reasons are that high harmonics of FFT frequencies may not superimpose with other strong frequencies around $F_{R}, 2 F_{R}$, or $F_{L}$ frequency in certain motors. Therefore, high harmonics sometimes are a good indicator to confirm a fault in induction motors. In a few cases such as in bearing related faults, early indication of these faults might be shown through frequencies higher than $10 \mathrm{KHz}$. However, as can be seen from many fault predictors, even with the vibration monitoring technique that most of them are concentrated on 10x to $20 \mathrm{x}$ of fundamental frequencies. Therefore, 
$1000 \mathrm{~Hz}$ of bandwidth combined with acceptable FFT frequency resolutions might be a good approach to achieve a balance between gaining comprehensive results and saving computation memories.

Table 3.1 Sensor parameters and positions

\begin{tabular}{|l|l|l|l|l|}
\hline $\begin{array}{l}\text { Monitoring } \\
\text { technique }\end{array}$ & \multicolumn{1}{|c|}{ Signals } & \multicolumn{1}{|c|}{ Sensors } & \multicolumn{1}{c|}{$\begin{array}{c}\text { FFT } \\
\text { Bandwidth }\end{array}$} & \multicolumn{1}{|c|}{$\begin{array}{c}\text { Position } \\
\text { (see Figure 3.18) }\end{array}$} \\
\hline Vibration & $\begin{array}{l}\text { Acceleration } \\
\text { Velocity } \\
\text { Displacement }\end{array}$ & $\begin{array}{l}\text { Accelerometer } \\
\text { (see Figure 2.4) }\end{array}$ & $\begin{array}{l}1-2 \mathrm{~g} \\
10 \mathrm{~Hz} \text { to 1 } \\
\mathrm{KHz}\end{array}$ & $\begin{array}{l}\text { Motor drive end and } \\
\text { non drive end (axial } \\
(1) \&(4), \text { radial- } \\
\text { horizontal (not shown } \\
\text { in Figure, radial- } \\
\text { vertical (2)\&(3)) }\end{array}$ \\
\hline Stator Current & Current & $\begin{array}{l}\text { CT, Hall Effect, and } \\
\text { Rogowski Coils } \\
\text { (see Figure 2.5) }\end{array}$ & $\begin{array}{l}1,000 \mathrm{~Hz}, 20 \text { th } \\
\text { Harmonic }\end{array}$ & $\begin{array}{l}\text { Clipped to one, two or } \\
\text { three phase line supply } \\
(5)\end{array}$ \\
\hline $\begin{array}{l}\text { Stator } \\
\text { Voltage }\end{array}$ & $\begin{array}{l}\text { Voltage } \\
\text { Transformers, } \\
\text { Differential Isolation } \\
\text { Amplifiers } \\
\text { (see Figure 2.6) }\end{array}$ & $\begin{array}{l}1,000 \mathrm{~Hz}, 20 \text { th } \\
\text { Harmonic }\end{array}$ & $\begin{array}{l}\text { Clipped to one, two or } \\
\text { three phase of supply } \\
\text { (5) }\end{array}$ \\
\hline $\begin{array}{l}\text { Flux Leakage } \\
\text { Monitoring }\end{array}$ & $\begin{array}{l}\text { Magnetic } \\
\text { Field }\end{array}$ & $\begin{array}{l}\text { Search Coil Hall } \\
\text { sensors (external or } \\
\text { internal) } \\
\text { (see Figure 2.8) }\end{array}$ & $\begin{array}{l}\text { Around mV, } \\
1,000 \mathrm{~Hz}, 20 t h \\
\text { harmonic }\end{array}$ & $\begin{array}{l}\text { Non drive-end or } \\
\text { motor out board } \\
\text { around the shaft(7) }\end{array}$ \\
\hline
\end{tabular}

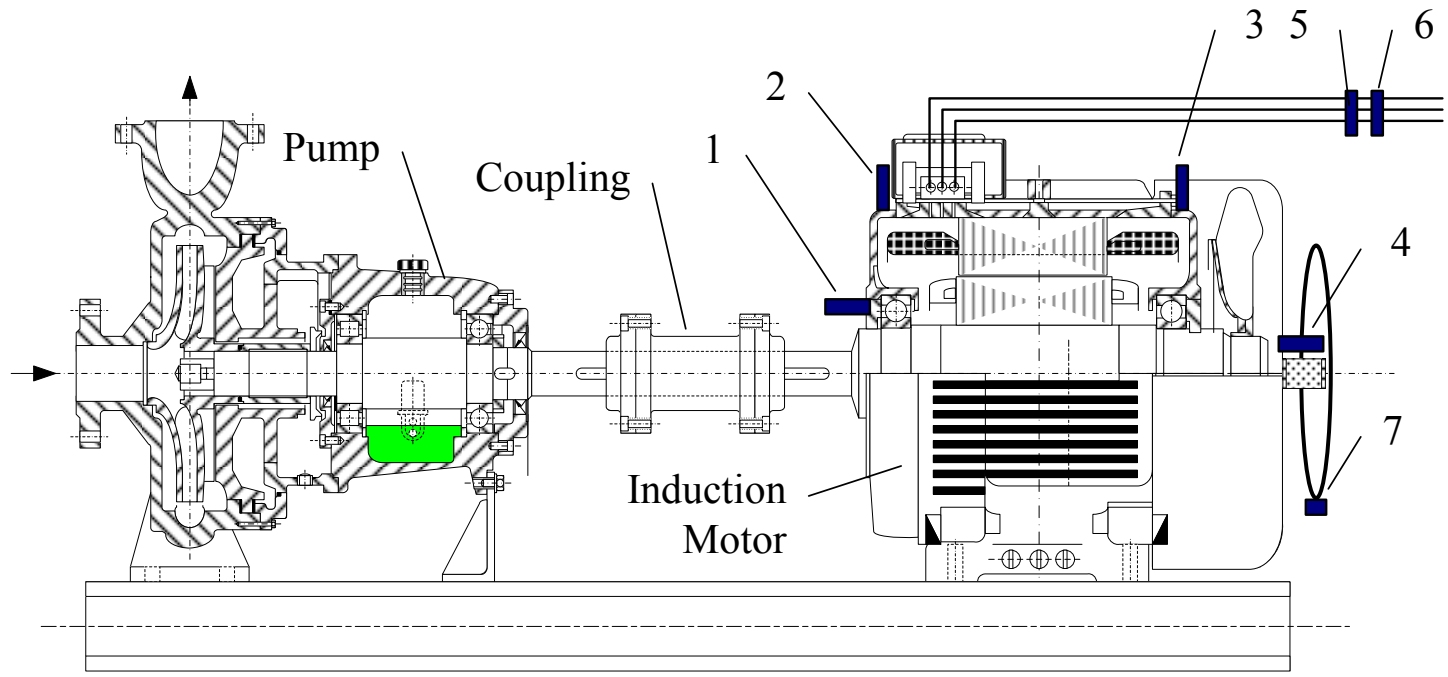

Figure 3.22 Potential position of sensors to gain comprehensive result of condition monitoring when a motor is coupled with a load

Figure 3.22 shows the potential positions of the sensors for typical condition monitoring applications. Vibration monitoring can be done using vibration sensors that are mounted on drive end or the non-drive end of induction motors. For 
comprehensive monitoring, the accelerometers are installed as close as possible with the two bearings on radial, axial direction or both. Sometimes, in order to obtain magnitude of vibration in all directions, the accelerometers are mounted on horizontal, vertical or axial directions. In the case of stator current or voltage monitoring, stator and voltage transducers are connected to two or all power leads. The external search coil for flux leakage monitoring can be attached to a motor non drive end around the shaft. This discussion about the sensors indicate that at least eight signals might be needed for condition monitoring, three or two signals for current, three or two signals for voltage, one or two for vibration, and one signal for flux monitoring. This means that for the proposed condition monitoring system at least an eight channel data acquisition of an analog input unit is needed.

\subsection{Conclusion}

This chapter began by providing an overview of induction motor structure, and the principle of operation. These basic principles provide valuable information to understand the effect of induction motor faults. Next, common faults of induction motor have been discussed including bearing, stator, rotor, eccentricity and set-up related faults. For each fault group, common specific problems, causes of failure and fault frequencies based on FFT analysis were discussed. These fault frequencies will be used as references to recognize peaks in the measured spectra.

As discussed in this chapter, a specific induction motor fault can be recognized through many approaches following studies established in these fields. It can be concluded that each approach offers solutions for a specific fault based on a specific monitoring technique of the FFT analysis results. Therefore, to provide general comprehensive information of common faults, implementation of many specific fault predictors will cover a wide range of faults. To obtain information about the common faults, this fault frequency analysis should be implemented in a suitable data acquisition, signal processing, and fault detection condition monitoring unit.

As mentioned in Chapter 2, this thesis considers the industry-standard CompactRIO architecture as the condition monitoring unit. This device can be located near or attached to an induction motor. The next chapter will describe the detailed development of this monitoring unit. 


\section{Chapter 4}

\section{Development of a CompactRIO- Based Condition Monitoring System}

\subsection{Introduction}

The CompactRIO is a reconfigurable hardware device that can offer highspeed parallel, and real-time processing tasks in the same unit. The device also accommodates LabVIEW programming [111] for the real-time processing under an FPGA environment.

As stated earlier in Chapter 2, the CompactRIO architecture is used in this thesis as a condition monitoring unit for an induction motor. In this chapter the detailed development of the CompactRIO based condition monitoring platform will be presented. The development will be segmented into three major developments, the CompactRIO indicator unit, the CompactRIO FPGA and the CompactRIO real-time controller software. In addition, to gain a better understanding of the CompactRIO development, an overview of the CompactRIO base unit and LabVIEW will be given first.

\subsection{Overview of CompactRIO and LabVIEW}

As shown in Figure 4.1 [89], currently there are four types of CompactRIO architecture: the embedded modular system, the embedded integrated system, the Rseries expansion system and the remote high-speed interface system. Both of the CompactRIO embedded modular system and the embedded integrated system rely on an FPGA reconfigurable system and a real-time controller. The real-time controller in the embedded integrated system is put together with the FPGA in a chassis allowing 
lower cost for high volume CompactRIO applications. However, for the embedded modular system, the real-time controller is separated from the FPGA chassis enabling the possibility of module upgrading for high performance and flexibility of module expansion. Furthermore, the CompactRIO R-series expansion and remote high-speed systems, are implemented basically when external processing capabilities that have high speeds and large amounts of data storage are demanded.

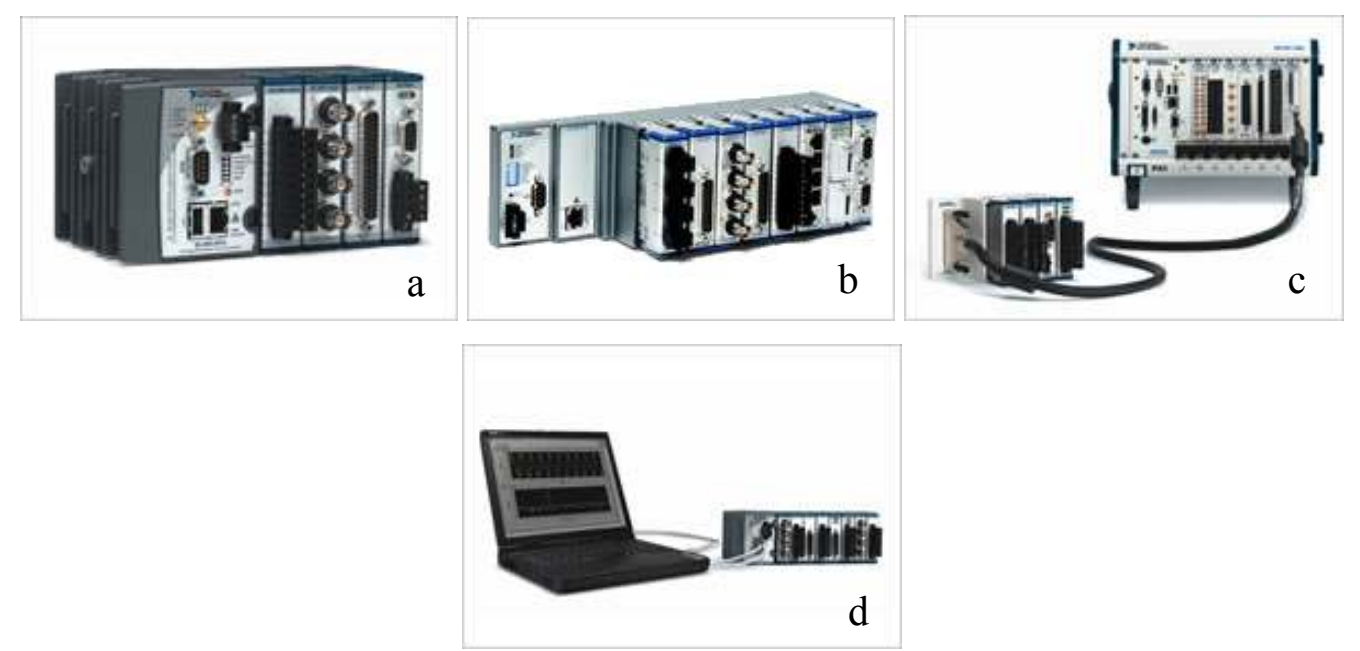

Figure 4.1 Four types of CompactRIO configuration: (a) embedded modular, (b) embedded integrated, (c) R-series expansion, and (d) remote high-speed

In this thesis the CompactRIO condition monitoring system follows the configuration of the reconfigurable embedded modular system. This configuration is chosen because this CompactRIO based unit offers a complete stand alone application, without additional PC or other external controller devices during operation. This platform meets the aim of this thesis to develop an on-site condition monitoring system next to induction motor. In all further discussion, the term CompactRIO in this thesis is associated with the type of CompactRIO embedded reconfigurable modular system. In this section, in order to gain an understanding of the CompactRIO condition monitoring system, general information regarding CompactRIO architecture and the segmented development of the compactRIO based condition monitoring system will be presented.

\subsubsection{CompactRIO Architecture}

The CompactRIO embedded system is a small and compact reconfigurable system, which is introduced to be implemented for high performance of industrystandard data acquisition and control applications [89]. To achieve this performance, 
the CompactRIO integrates a reconfigurable FPGA and a real-time processor that can be implemented for stand alone embedded or distributed applications. Furthermore, the CompactRIO accommodates custom-made I/O modules according to the user's applications or various hot-swappable I/O modules which have built-in signal conditioning from National Instrument Corporation. These industrial I/O modules can be connected directly to various sensors and transducers. The CompactRIO architecture is illustrated in Figure 4.2.

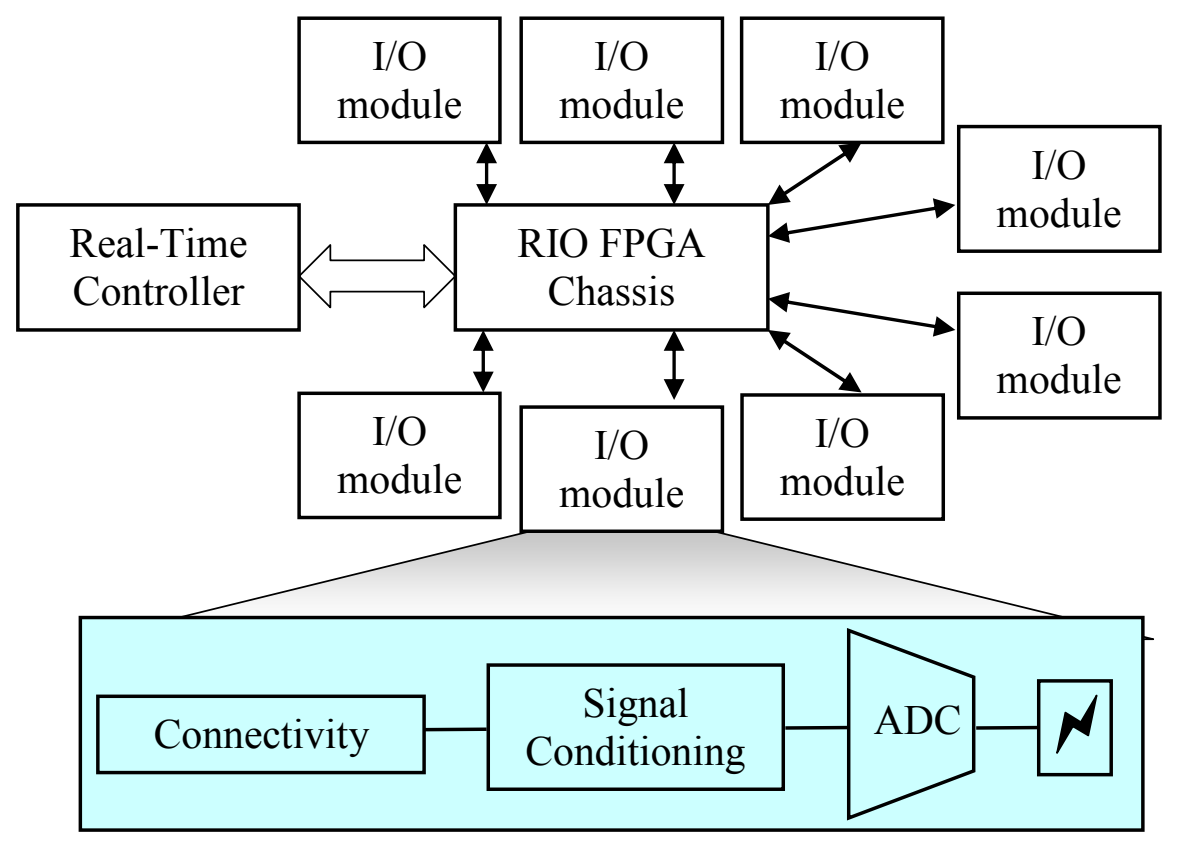

Figure 4.2 CompactRIO embedded architecture

Figure 4.2 shows that the CompactRIO embedded system can be developed based on the combination of a Reconfigurable Input Output (RIO) FPGA chassis, a Real-Time Controller, and one or more I/O modules in a chassis. This configuration offers open access and development flexibility to low-level hardware resources, both for the FPGA and I/O modules. This direct hardware access is achieved using elemental LabVIEW functions which will be explained later.

The RIO FPGA performs direct hardware access to the input/output circuitry of each I/O module as shown in Figure 4.2. This FPGA, which is connected independently with each $\mathrm{I} / \mathrm{O}$ module in the star topology of four or eight slots, can perform local integer based signal processing and pass signals directly from one module to another. In other words, this topology also enables flexibility in timing and triggering of each I/O module. Due to this star configuration, the $\mathrm{I} / \mathrm{O}$ operation of each module can be synchronized to within 25 ns resolution. Each I/O module consist of 
optional isolation barrier, ADC or DAC, signal conditioning and connectivity to meet the requirements of various applications.

Figure 4.2 illustrates that the RIO FPGA architecture functions as a core of the platform to transfer the data to a real-time embedded processor in the real-time controller. This connection can be done through a PCI bus using specific protocols, which will be discussed later. The real-time controller then carries out real-time data analysis, post processing, data logging, or data communication with the network.

\subsubsection{CompactRIO Base Unit}

In order to meet flexible user applications three basic modules that are required for a CompactRIO base unit: CompactRIO real-time embedded controller, CompactRIO chassis and I/O modules. Examples of these modules are presented in Table 4.1.

As can be seen from this table, the CompactRIO real-time embedded controllers feature DRAM memory, non-volatile flash storage memory, and an embedded real-time processor, with a clock speed $200 \mathrm{MHz}$ or $400 \mathrm{MHz}$. These realtime controllers, which are designed for extreme industrial environments and low power consumption, are also able to supply $9 \mathrm{~V}$ to $35 \mathrm{~V}$ to the chassis and $\mathrm{I} / \mathrm{O}$ modules. In addition, these real-time controllers are fitted with an Ethernet connection and built-in HTTP and FTP servers. The controllers are also equipped with an RS-232 serial connector for a keypad, LCD display or barcode reader. All these features offer balanced powerful real-time floating-point signal processing and data communication, as well as maintaining low power consumption.

The second important module shown in Table 4.1 is the CompactRIO reconfigurable FPGA chassis which is the heart of the base unit. The size of FPGA is a $1 \mathrm{M}$ or $3 \mathrm{M}$ gate of embedded FPGA logic that can be used for the custom reconfigurable applications. Similar to the real-time controller, the FPGA chassis which has a rugged metal construction is also designed following industrial standards to firmly hold I/O modules and the real-time controller during operation. 
Table 4.1 Modules of CompactRIO embedded system [112]

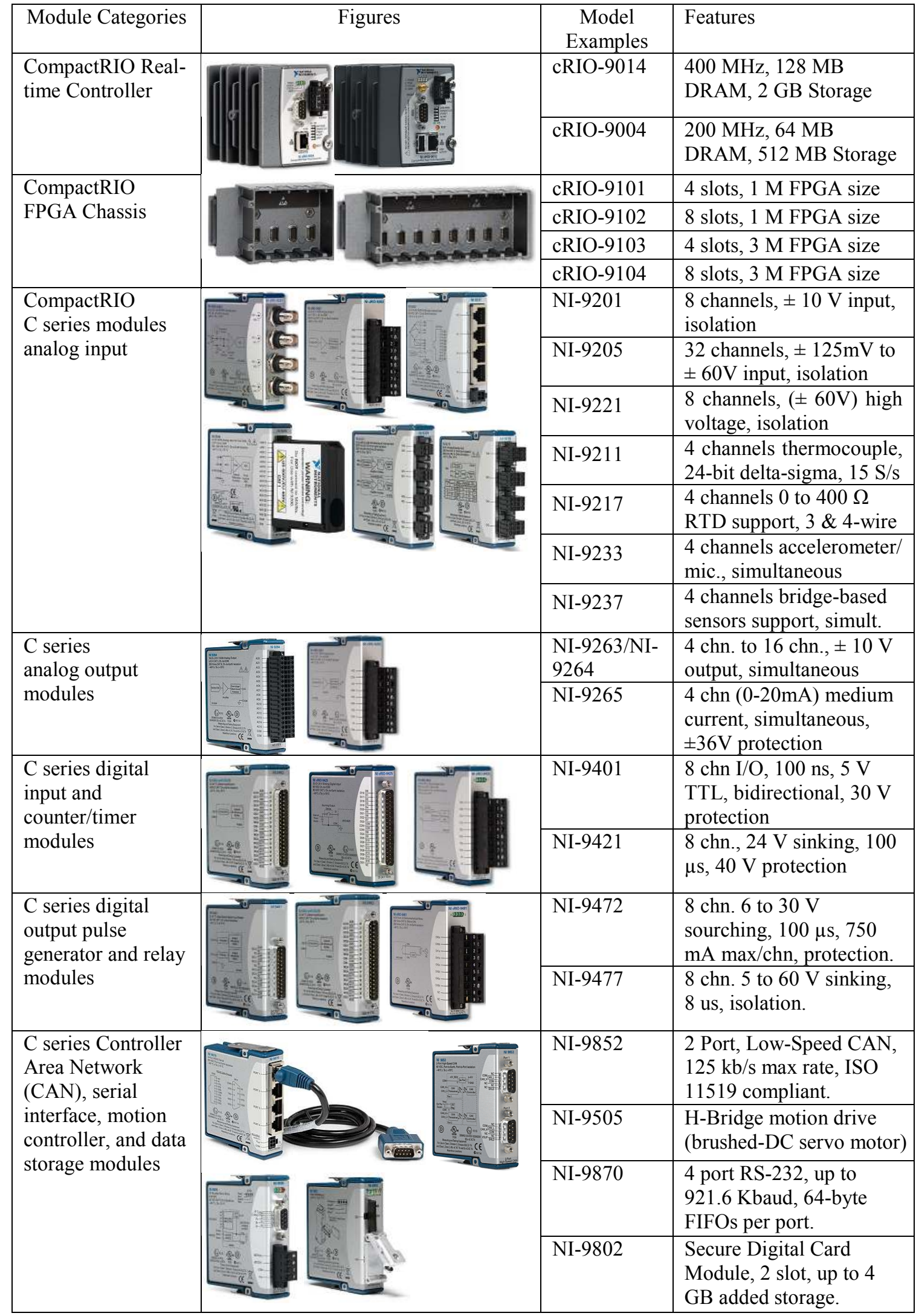


The last module is the $\mathrm{C}$ series $\mathrm{I} / \mathrm{O}$ modules that can be selected according to the specific application. The National Instrument Corporation provides various types of I/O module such as those listed in Table 4.1, for example, $\pm 80 \mathrm{mV}$ thermocouple input, $\pm 10 \mathrm{~V}$ simultaneous sampling analog inputs/outputs, $24 \mathrm{~V}$ industrial digital $\mathrm{I} / \mathrm{O}$ with up to $1 \mathrm{~A}$ current drive, differential/TTL digital inputs with $5 \mathrm{~V}$ regulated supply output for encoders, CAN controller, and serial interface. Each of these I/O modules has built-in signal conditioning and has a screw terminal or D-Sub connector allowing a direct connection to the sensors or actuators. In addition to the available $\mathrm{I} / \mathrm{O}$ modules, the National Instruments Corporation also provides the flexibility of custom development of an I/O module kit to meet any flexible requirements. This custom development of an I/O module will be discussed in the following section. Further details of the module types and a selection guides can be found in [112].

\subsubsection{CompactRIO Module Development Kit}

The CompactRIO Module Development Kit (MDK) [113] is provided to expand the capability of the CompactRIO in order to meet the unique needs of particular applications or products. This kit comprises ten empty module shells, development software and a variety of header connectors, such as D-Sub, BNC and screw terminals shown in Figure 4.3.

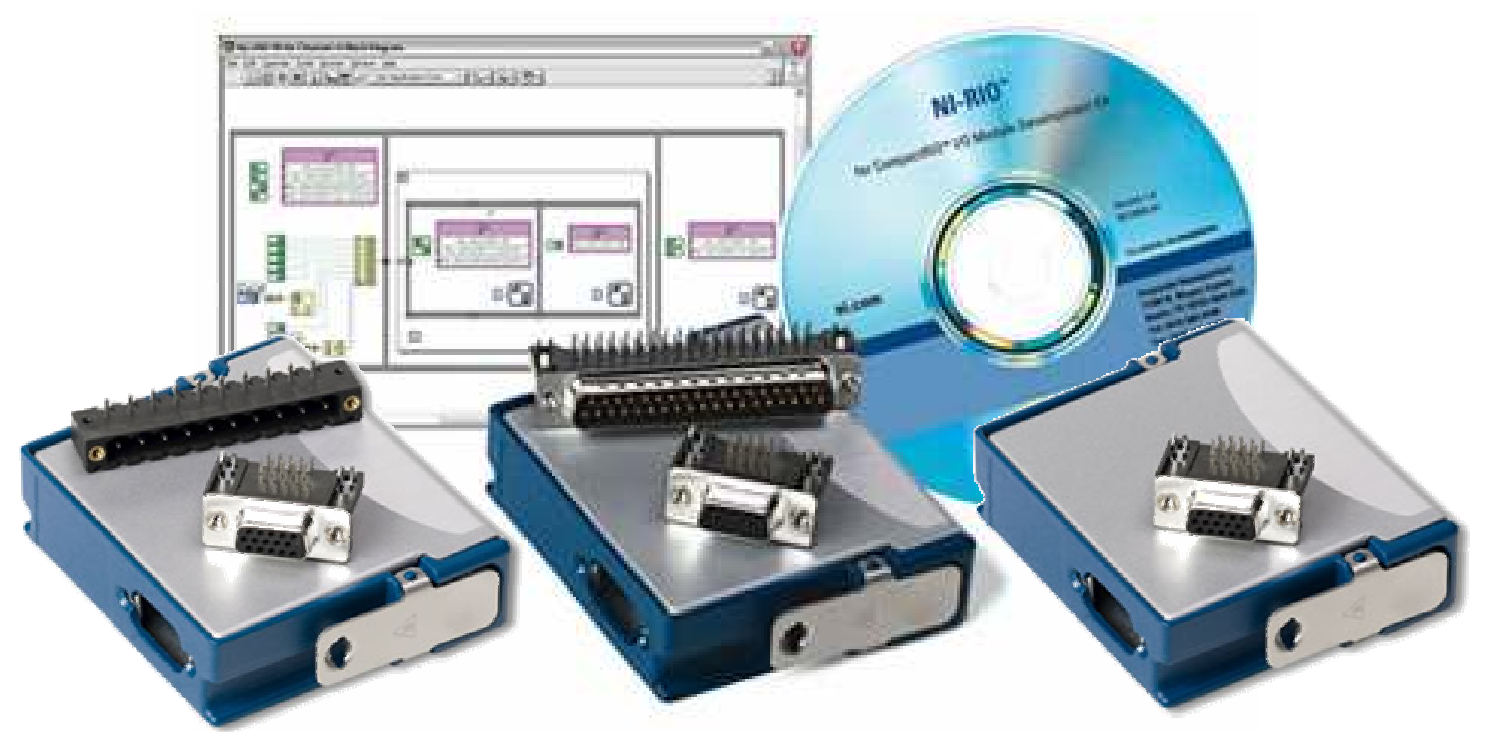

Figure 4.3 CompactRIO Module Development Kit shows empty custom module shells, and development software

CompactRIO MDK also presents the hardware requirements and recommendations, EEPROM format, and development of VI drivers for the I/O 
modules. The CompactRIO MDK also licenses users to develop and manufacture their own CompactRIO modules. Detail of the development of custom made CompactRIO, known as cRIO-generic, will be presented in the section on custom indicator modules.

\subsubsection{LabVIEW Programming}

As mentioned in the Introduction, CompactRIO programming will be carried out using LabVIEW software - Laboratory Virtual Instrument Engineering Workbench. LabVIEW provides a software layer that can be interfaced easily to the hardware layer of the hardware products of National Instrument (NI) and its alliance partners without the need to understand complex hardware machine languages. LabVIEW programming language provides a graphical development environment using dataflow technique to represent the connectivity of each module or function included. These modules and functions can be built from a basic block of LabVIEW known as Virtual Instruments (VI).

The LabVIEW VIs consist of two main parts: a front panel where a user interface can be designed, and a block diagram where the graphical source codes are created as shown in Figure 4.4. These VIs can be obtained from the available function palette and add-on tools provided for LabVIEW, or can be developed according to specific applications needed. In addition, the LabVIEW platform, as an open language, enables these VIs to be connected with other existing applications through .NET, ActiveX, DLLs and networking protocols. These VIs can be managed through a project explorer, which also can be utilized to manage multiple targets such as a realtime or embedded hardware. Furthermore, in order to display a user-friendly interface, fully customized graphs, buttons, tables, and indicators are available in the control palette.

As mentioned before, LabVIEW development software has several add-on modules for specific target environments or purposes, for example LabVIEW PDA, LabVIEW DSP, LabVIEW Real-time and FPGA. The LabVIEW development system as the core software is also supported by several add-on toolkits to expand the functionalities, for example Advanced Signal Processing Toolkit ${ }^{\mathrm{TM}}$ for specific signal processing applications, and Internet Toolkit ${ }^{\mathrm{TM}}$ for advanced distributed systems. 


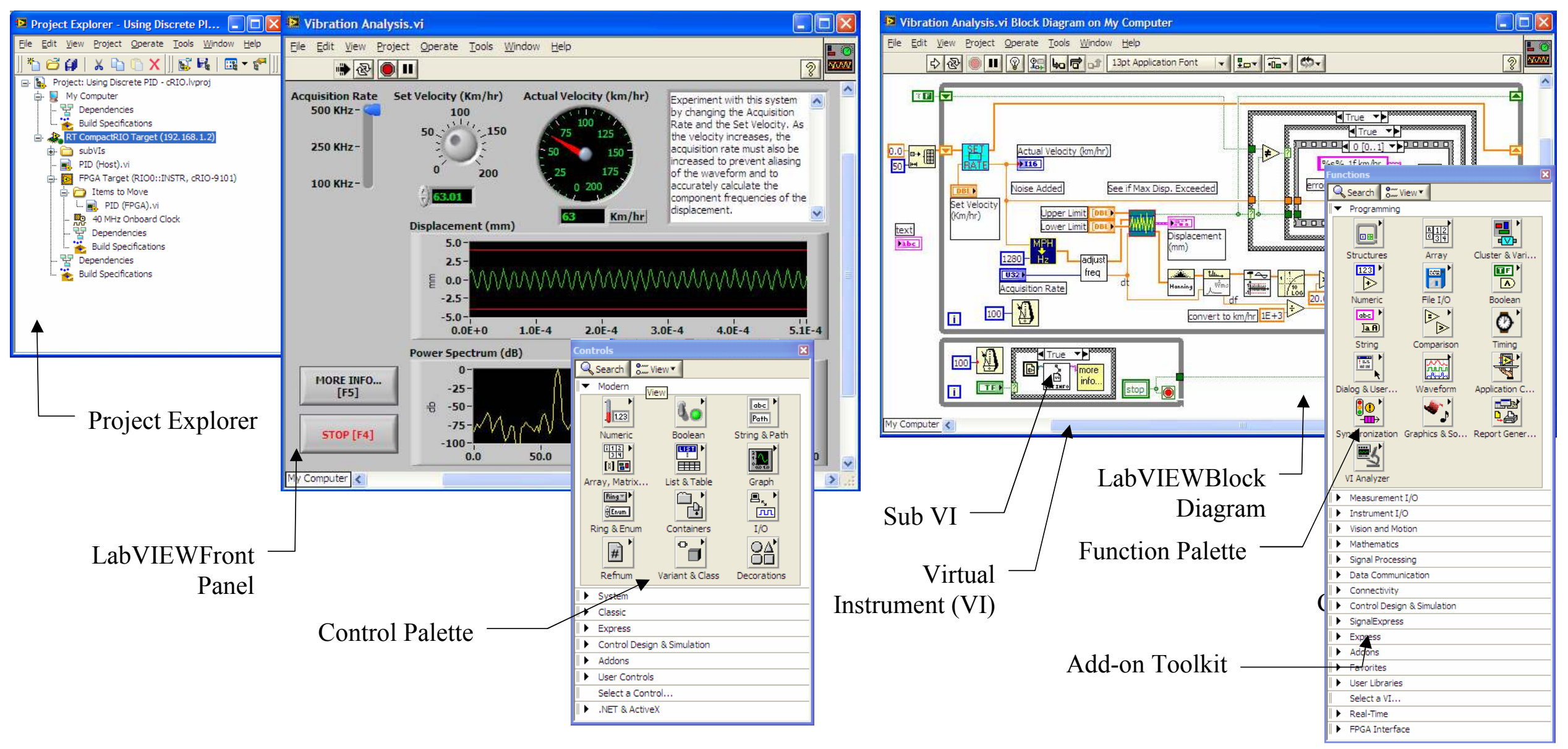

Figure 4.4 LabVIEW programming components 
The CompactRIO base unit comprises an FPGA and real-time controller. To cope with these hardware system, LabVIEW provides specific software for each development: LabVIEW FPGA for FPGA development, and LabVIEW Real-time for real-time applications. Considering these environments, therefore, in the next section of this thesis the CompactRIO segmented development is divided according to the specific environment of LabVIEW software employed and the CompactRIO module types.

\subsubsection{CompactRIO Condition Monitoring Segmented Development}

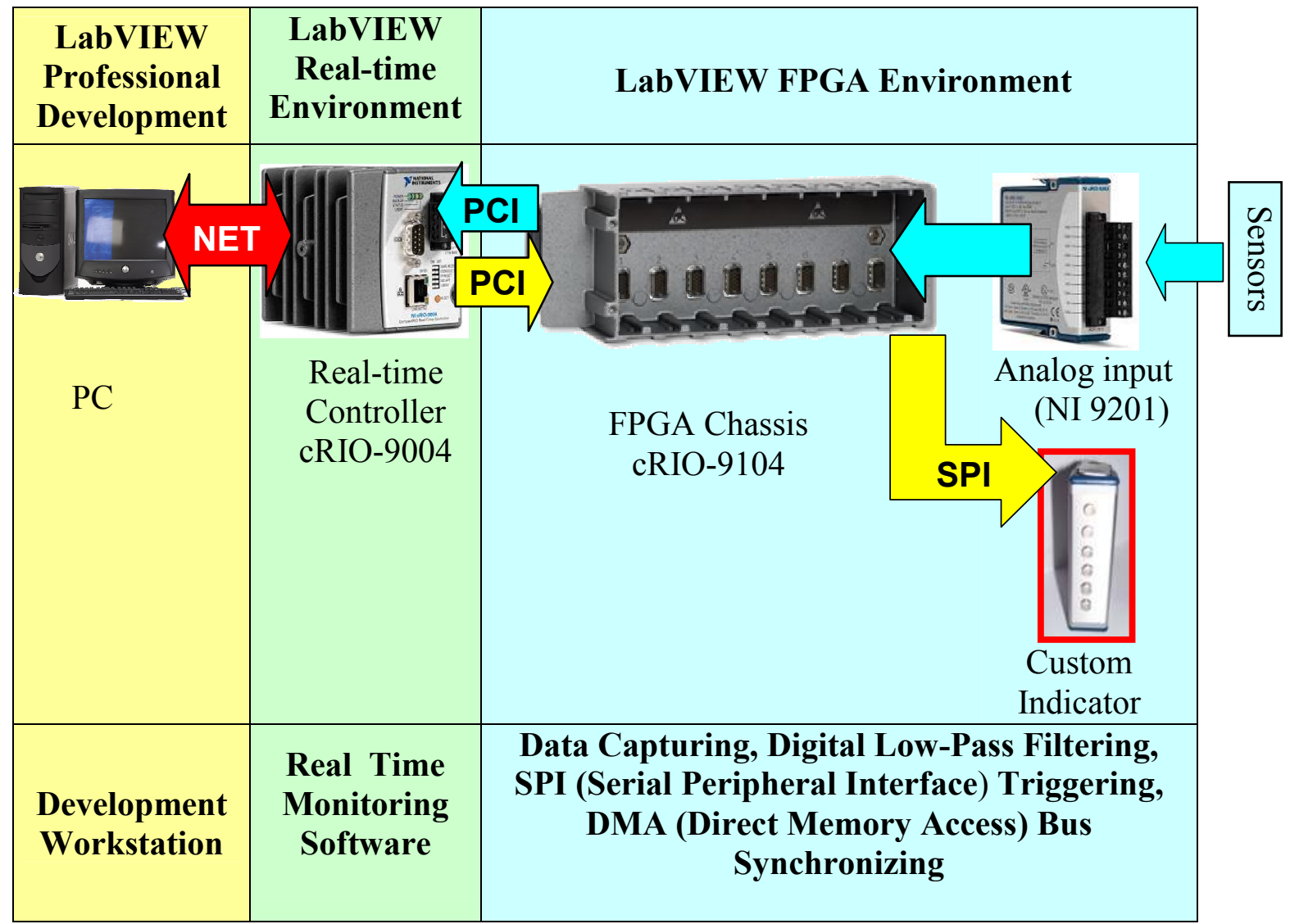

Figure 4.5 Segmented development of CompactRIO embedded condition monitoring

Following the arrangement of the CompactRIO base unit mentioned previously, three main modules were selected for the CompactRIO-based condition monitoring. These modules are a real-time embedded controller module (cRIO-9004); a reconfigurable chassis containing user programmable FPGA (cRIO-9104); and an analog input module (NI 9201) as illustrated in Figure 4.5. Another important module 
shown in this figure is a custom-made CompactRIO indicator module. As has been stated before, its development is one of the aims of this thesis.

Following the specific target environment of the CompactRIO system, as classified in Figure 4.5, development is undertaken according to the different types of environment of the LabVIEW software. The first development is performed under an FPGA environment where LabVIEW FPGA will be involved and an analog input NI9201 or custom indicator module will be driven.

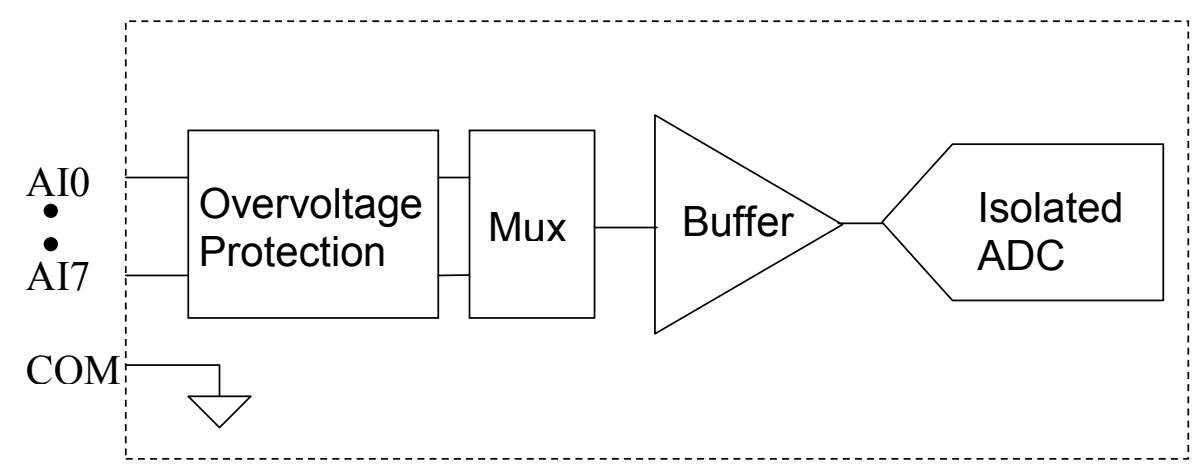

Figure 4.6 Input circuitry of NI 9201

As illustrated in Figures 4.5 and 4.6, an analog input module NI 9201 (Cseries, 8-channels, 12 bit SAR ADC, $500 \mathrm{kS} / \mathrm{s}$ ) [114] is used to capture vibration, current, voltage and flux leakage signals using suitable sensors and signal conditioning devices. The total input channels meet the requirement of sensory signals which are employed for the condition monitoring system. This module is suitable for condition monitoring application because it has 8 analog input channels to capture $10.8 \mathrm{~V}$ maximum operating voltage signals. In addition, this module also contains channel-to-earth ground isolation barrier up to $100 \mathrm{~V}$, isolated ADC, and noise immunity system, which are desirable features in signal measurements in condition monitoring. Detail of the sensors and connection arrangement will be presented in the next section.

In this research the capacity of the programmable FPGA cRIO-9104 (Xilinx Virtex II XC2V3000-4FG676I, 3 million gate sizes, 8 I/O module slots) chassis is utilized widely, including the signal processing function such as implementing low pass digital filtering routines. Furthermore, the FPGA chassis also reads sensor signals directly from the analog input module. The FPGA also writes the status of the motor to a custom design indicator module through SPI triggering technique, as will be 
explained later in this chapter. While the FPGA is capturing data, it also transfers data through the PCI bus to the real-time host controller. Several techniques can be implemented for this host synchronization such as polling, interrupt request and direct memory access. These functions can be programmed through the LabVIEW FPGA environment.

The second development environment is carried out under the LabVIEW Realtime environment, shown in Figure 4.5. The CompactRIO real-time embedded controller (cRIO-9004) has the capacity to perform real-time stand-alone execution applications. To support these capabilities, the real-time controller provides $512 \mathrm{Mb}$ of nonvolatile storage memory, $64 \mathrm{Mb}$ RAM and $200 \mathrm{MHz}$ Pentium processor. For this research, the Ethernet port is connected to a PC as a re-engineering and development station for uploading FPGA bit files and condition monitoring software. Therefore, based on these potential capabilities, the CompactRIO real-time controller is employed to perform real-time execution of the condition monitoring application including windowing, FFT analysis, averaging, peak detection and fault analysis.

A custom-made CompactRIO indicator module, which is basically not part of the CompactRIO based unit, is also developed in this research. This module functions as a simple indicator using (Led Emitting Diodes) LEDs, as shown in Figure 4.5. This module utilizes Serial Peripheral Interface (SPI) protocol to communicate with the FPGA. Details of the design and development will be presented in the following section.

\subsection{The CompactRIO Indicator Module}

A custom-designed indicator module is designed to provide an on-site fault diagnosis for the operator. The main function of this module is to provide information about the induction motor to the operators nearby concerning various groups of faults and associated fault levels. To present simple information for the operators, the indicator module is designed using LEDs which indicate general severity levels of each fault.

\subsubsection{Basic requirements}

As mentioned in the section on segmented development, this LED-based indicator module must be able to be recognized and driven from the FPGA chassis. 
Therefore, the indicator module must meet several requirements of the CompactRIO Module Development Kit (MDK) such as mechanical, physical, electrical, EEPROM, power and ground module interface signals, safety standards, environmental, shock and vibration, and electromagnetic compatibility standards. In this thesis, electrical and communication modes, power and ground will be discussed in the following sections, while further information on other aspects can be found in the CompactRIO MDK User Guide.

\subsubsection{Electrical and Communication Modes}

In the case of the electrical and communication modes, CompactRIO MDK recommends that custom modules follows either the ID mode or Operation Mode as illustrated in Figure 4.7 [113]. The ID mode requires EEPROM delivering information about the identification and description of the CompactRIO module such as vendor ID, product ID, and serial number to the CompactRIO base unit. This technique is commonly implemented for commercial CompactRIO products.

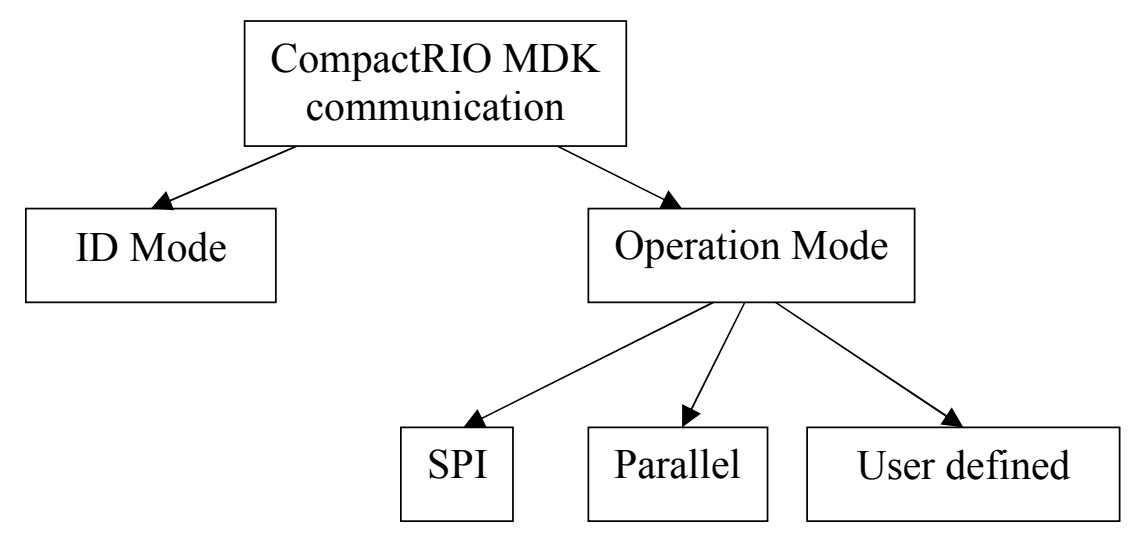

Figure 4.7 CompactRIO communication mode

The Operation mode can be used for direct access to $\mathrm{I} / \mathrm{O}$ circuitry of the custom module. This mode supports the SPI communication template, parallel mode, and user-defined templates. The SPI template, including additional signals for timing and triggering, provides serial communication between a complex I/O circuitry and the CompactRIO base unit. Furthermore, when basic digital I/O or other simple direct $\mathrm{I} / \mathrm{O}$ is needed, the parallel template can be implemented. When both these templates of operation mode cannot meet the applications needed, the user-defined mode might be implemented. 
Table 4.2 Key pins of the SPI template in Operation Mode of CompactRIO custom module development [113]

\begin{tabular}{|l|l|l|l|}
\hline Pin & \multicolumn{1}{|c|}{ Signal } & Direction & \multicolumn{1}{c|}{ Description } \\
\hline 2 & SPI_CS & Input & SPI chip select port (active low) \\
\hline 4 & Power & Input & 5V DC \\
\hline 6 & MOSI & Input & Master-Out Slave-Input SPI data line \\
\hline 11 & SPI_CLK & Input & $\begin{array}{l}\text { SPI data clock that is used for synchronization with SPI } \\
\text { device }\end{array}$ \\
\hline 12 & MISO & Output & Master-In Slave-Out SPI data line \\
\hline 14 & GND & Input & Ground reference for all SPI mode and ID mode signals \\
\hline
\end{tabular}

The operation mode is preferred for implementation in the CompactRIO indicator module. The SPI template is chosen to interface the CompactRIO indicator module with the FPGA chassis. This is because the SPI interface is suitable to reduce the complexity of communication to drive many LEDs. Table 4.2 above shows several key pins which can be used for SPI implementation in the CompactRIO platform.

\subsubsection{Power and Ground Signals}

In terms of power and ground signals, a custom module of the CompactRIO system should consider the key points to be implemented: average power consumption of a CompactRIO module, hot insertion, and ground signals [113].

The CompactRIO MDK recommends that power consumption must not exceed an average of $125 \mathrm{~mA}$ over any $100 \mathrm{~ms}$ period. In addition, the peak power consumption also must be under $150 \mathrm{~mA}$ by the time $150 \mu \mathrm{C}$ has been achieved. These parameters, which are also known as in-rush current, can be used as criteria for the hot insertion capability. These requirements are not difficult to be pursued, as long as too much total capacitance on the supply line circuit and a large number of ICs that draw high current during power-on can be avoided. Another requirement is that the power ground line of the custom module must be connected to the FPGA chassis. These implementations are explained in the following sections.

\subsubsection{Module Development}

The basic design of the CompactRIO indicator module can be seen in Figure 4.8. This block diagram explains three major parts of this module: a DSUB 15pin connector, LED drivers circuitry, and six LEDs. A D-Sub connector is connected with an FPGA chassis, where signals are driven. The LED driver circuitry then powers on 
LEDs according to the FPGA signals. The total number of LEDs is chosen according to the six common fault types in induction motors.

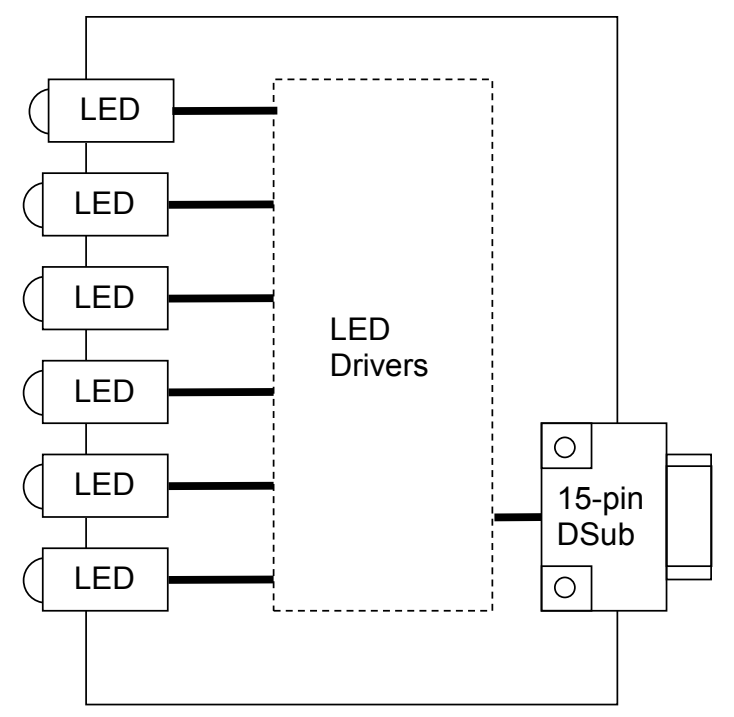

Figure 4.8 CompactRIO basic block diagram

In order to implement the basic block of the LED indicator module, the indicator module employs a specific LED driver and bi-colour LED type. The schematic diagram of the indicator module is described in Figure 4.9.

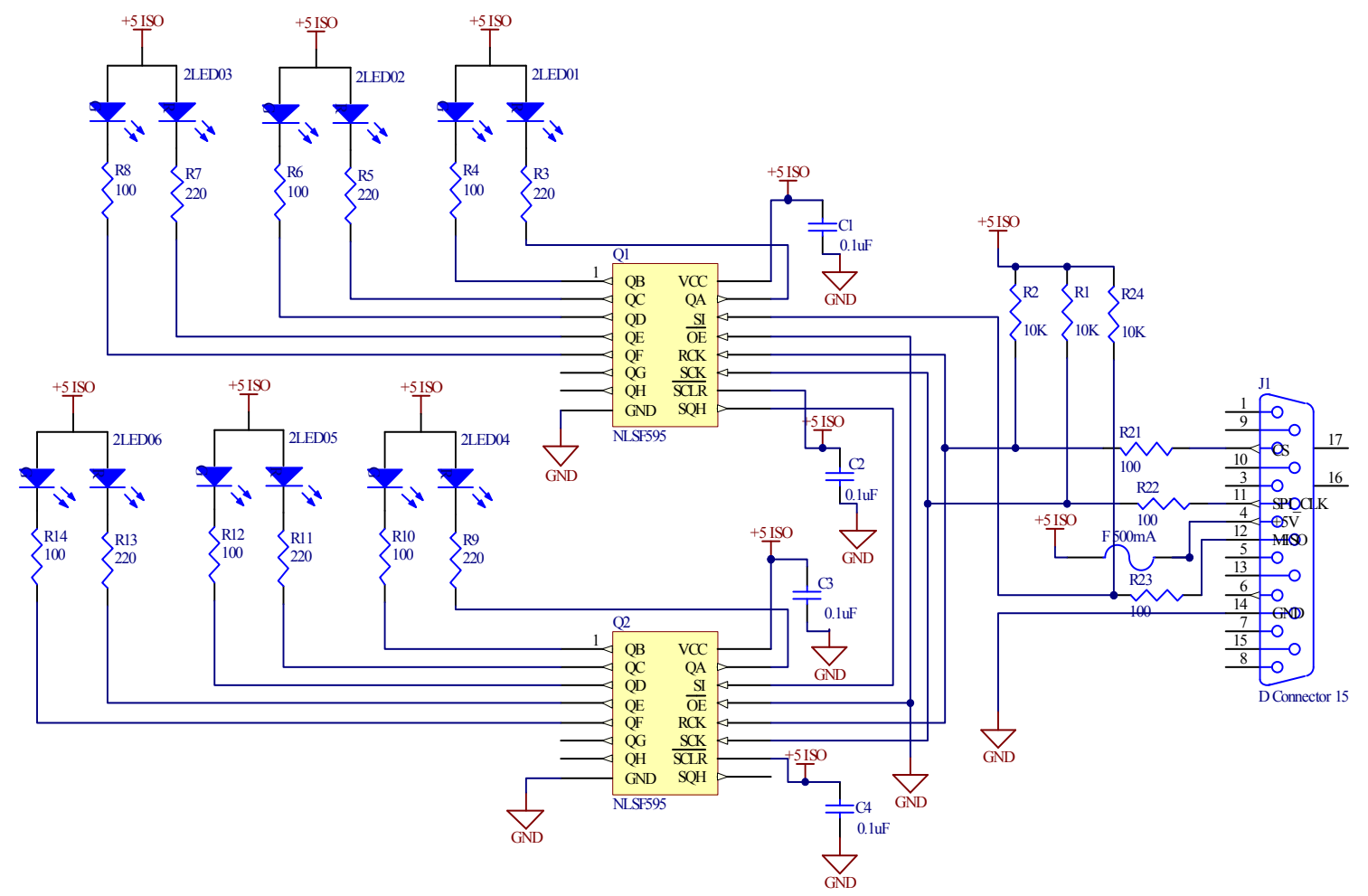

Figure 4.9 Schematic diagram of the CompactRIO indicator module 
As can be seen in Figure 4.9, the indicator module consists of three main parts: six bi-colour LEDs, two NLS595 LED drivers, and a 15 pin D-SUB connector. Each LED stands for a group of failures, such as bearing, rotor, stator, eccentricity, set-up and voltage problems. The bi-colour LEDs produce 3 colours: red, green and yellow (mix of red and green colours). The SPI bus is implemented through D-Sub highdensity 15 pin connector to the FPGA chassis.

The bi-colour LEDs and NLSF595 LED driver are chosen to reduce circuit complexity. The NLSF595 LED drivers also enable the FPGA to control the circuit through the SPI bus connection. Furthermore, the LEDs drivers which have SMD type components can decrease power consumption of the module and allow a higher packing density. The total power consumption of the module, when all LEDs with yellow colours are on, is less then $125 \mathrm{~mA}$. This is sufficient for the operation of the circuit.

Each of the NLSF595 LED drivers in Figure 4.9 operates three common anode bi-colour LEDs. Typically, each LED driver can drive up to four bi-colour LEDs [115]. In this design, to maintain brightness and ensure appropriate design for six LEDs, each of the NLSF595 LED drivers is engaged to drive three bi-colour LEDs.

One of the common techniques to run these LEDs is to use either a high or a low side switch. Each pin of eight bit parallel output of NLSF595 sinks more than 12 $\mathrm{mA}$. The typical schematic diagram of this DC LED drive technique is indicated in Figure 4.10.

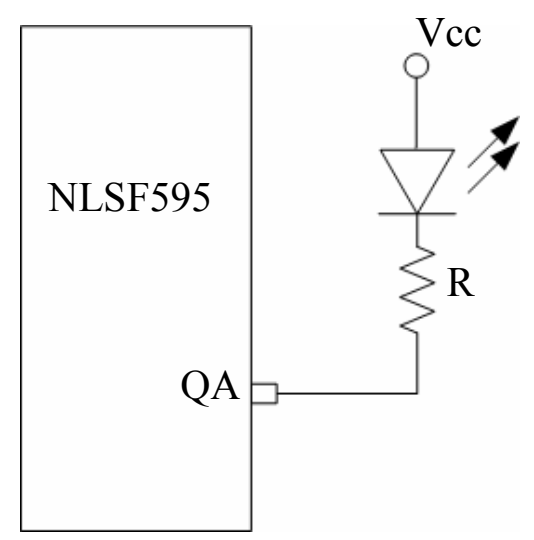

Figure 4.10 The LED drive technique

This technique is implemented by adjusting proportional current through diode. This current also determines the brightness of a LED. Ohm's Law, given in Equation. 4.1, is applied to obtain resistor $(\mathrm{R})$ values. 


$$
R=\frac{V_{C C}-V_{f, L E D}-V_{O L}}{I_{O L}}
$$

Where

$\mathrm{R} \quad=$ resistor value

$\mathrm{V}_{\mathrm{CC}} \quad=$ power supply voltage

$\mathrm{I}_{\mathrm{OL}} \quad=$ desired LED current

$\mathrm{V}_{\mathrm{f}, \mathrm{LED}}=$ LED forward voltage at $\mathrm{I}_{\mathrm{OL}}$

$\mathrm{V}_{\mathrm{OL}}=$ output low voltage from the LED driver at $\mathrm{I}_{\mathrm{OL}}$

The equation above considers the LED forward voltage drop and the output voltage of the LED driver when sinking current. For $\mathrm{V}_{\mathrm{CC}}=5 \mathrm{~V}, \mathrm{~V}_{\mathrm{f}, \mathrm{LED}}=2 \mathrm{~V}(\mathrm{red})$, $\mathrm{V}_{\mathrm{OL}}=0,25 \mathrm{~V}, \mathrm{I}_{\mathrm{OL}}=12 \mathrm{~mA}$, the nominal value of the resistor is $229.2 \mathrm{ohm}$. The closest common value implemented in this project is $220 \mathrm{ohm}$. For a super bright green colour, where $\mathrm{V}_{\mathrm{f}, \mathrm{LED}}=3.5 \mathrm{~V}$ [116], the nominal value of the resistor is $104.2 \mathrm{~V}$ $\mathrm{ohm}$. In this thesis the resistor used is $100 \mathrm{ohm}$ as the closest nominal value of an available commercial resistor.

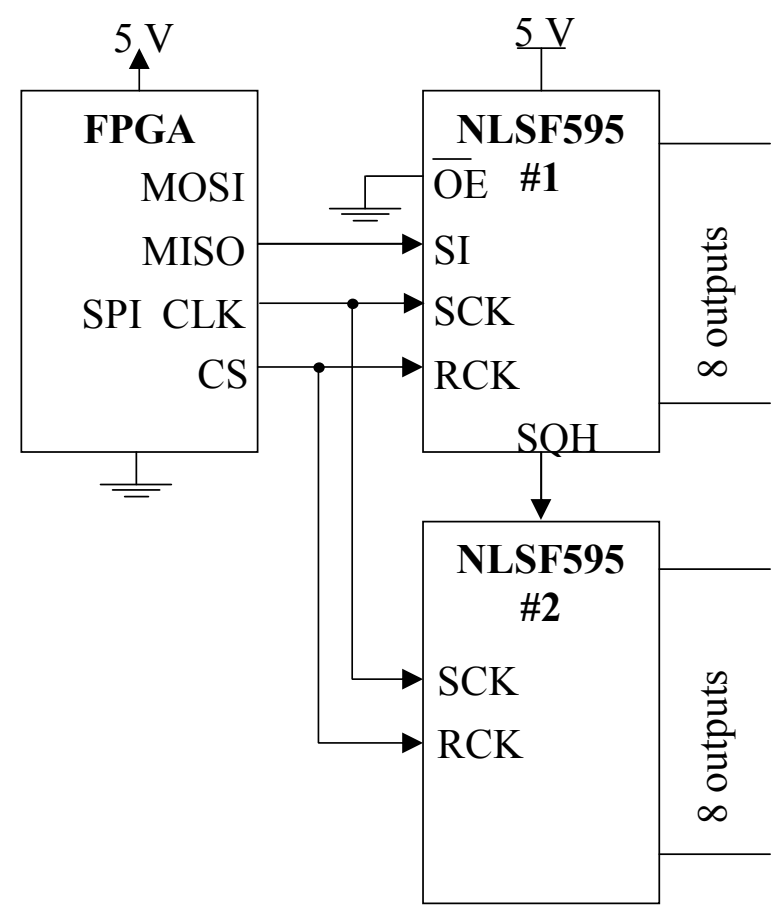

Figure 4.11 The SPI drive technique

As described previously, the indicator unit is controlled by the FPGA chassis through Serial Peripheral Interface (SPI) protocol. The SPI is one of the serial 
synchronous communication techniques implemented in physical layers or in component levels [117]. The SPI communication extension is useful because the FPGA provides only a few I/O channels in each chassis slot. In addition, the SPI has several advantages: it is fast, simple, low-cost, and universally accepted. The schematic connection of the SPI between the FPGA chassis and two NLSF595 drivers is shown in Figure 4.11.

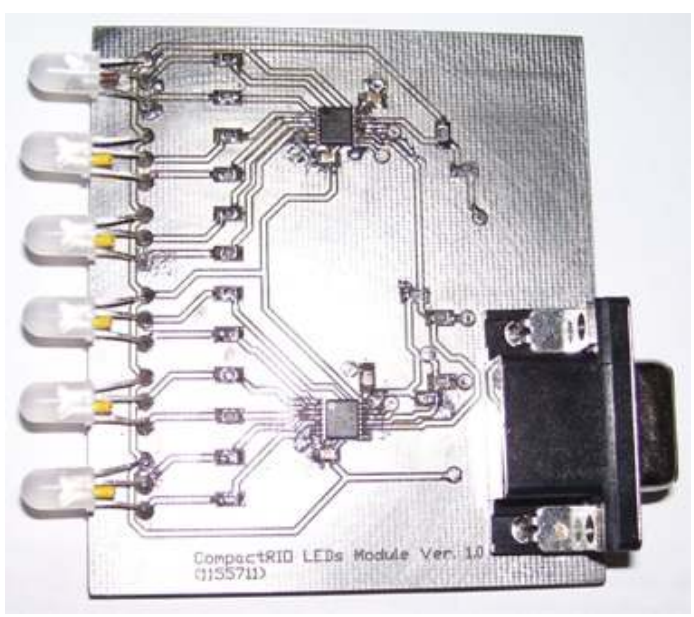

(a)

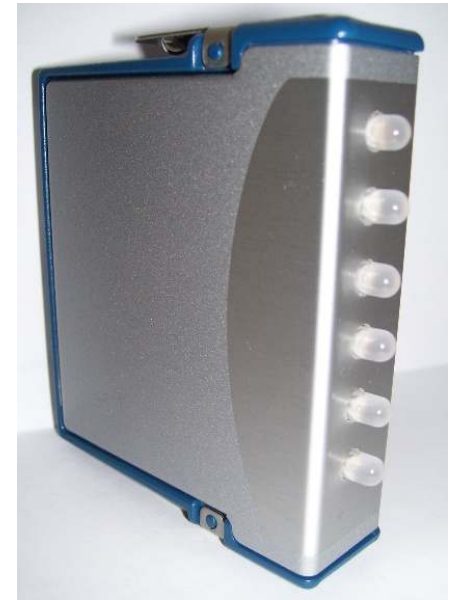

(b)

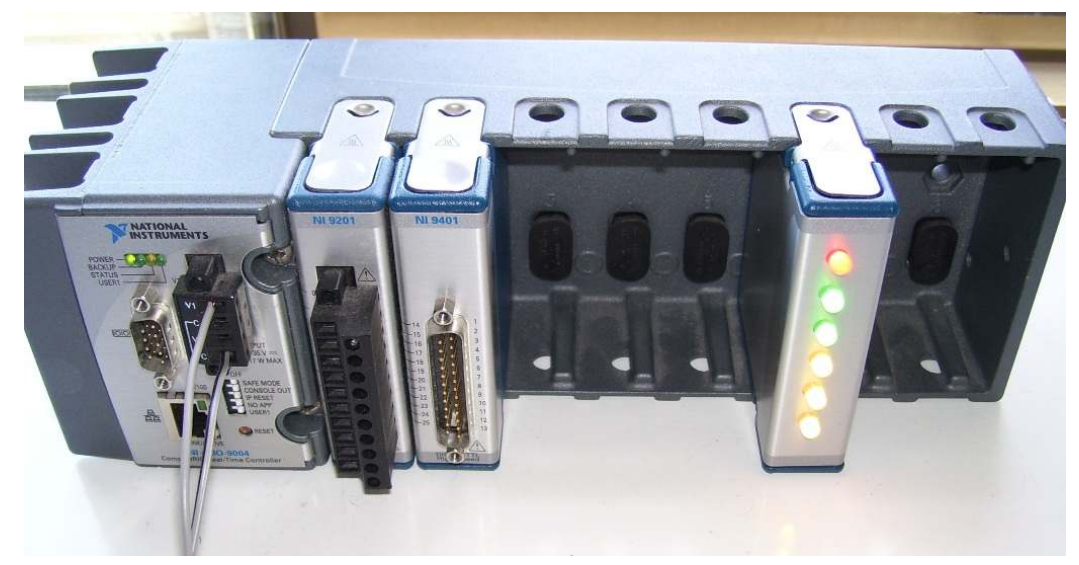

(c)

Figure 4.12 (a). CompactRIO indicator circuit, (b) CompactRIO indicator module, and (c) CompactRIO indicator module attached on CompactRIO base unit

As illustrated in Figure 4.11, the SPI technique requires two control lines (CS and SPI_CLK) and two data lines, Master-Out-Slave-In (MOSI) and Master-In-SlaveOut (MISO). The NLSF595, interestingly, requires an external controller, in this case the FPGA, connected through the MISO mode. The common external output devices are driven through the MOSI mode. The FPGA, which works as a master device, provides clock signals through an SPI_CLK port or a shift clock (SCK) in NLSF595. 
The FPGA also activates the slave components from a chip select signal to the shift register clock (RCK). Two NLSF595s are treated as a single slave for driving the six LEDs at the same time. Therefore, the cascaded NLSF595 slaves are both connected to a single chip select signal of the master. The serial output (SQH) of the first NLSF595 is connected to the data input port (SI) of the second NLSF595. The clock signals (SPI_CLK) are brought to the slaves for a synchronization of the communication.

Figure 4.12.(a) shows the final design of the CompactRIO LED indicator module. the six bi-colour LEDs are on the left side, the two NLSF595 LED drivers are in the middle, and a D-Sub 15 pin connector is on the left side of the module. Figure 4.12.(b) shows the final CompactRIO indicator module. This is inserted into one of the expandable slots and performs a full configuration of the CompactRIO condition monitoring for induction motors, such as in Figure 4.12.(c) This indicator module is driven by the cRIO-9001 FPGA chassis. The development of this FPGA will be discussed in the following section.

\subsection{FPGA Development}

The CompactRIO programmable FPGA is an essential part of the CompactRIO based condition monitoring project. The FPGA performs analog signal reading routines from the NI 9201 analog input module, signals filtering, signals delivering to the CompactRIO real-time host controller, and output writing to the custom-made indicator module. These tasks employ the FPGA capability to meet time critical requirements for an acceptable real-time condition monitoring device.

Figure 4.13 illustrates a flowchart of the developed FPGA which is programmed using LabVIEW FPGA ${ }^{\mathrm{TM}}$. As can be seen in this figure, the FPGA unit performs two main functions simultaneously, acquiring the eight channel analog signals and writing signals through the SPI bus to the custom-made indicator module. In other words, both branches are independent of each other.

This flowchart illustrates the eight channel calibration, followed by a sampling-rate reading. The channel calibration constants are needed to convert the binary $\mathrm{A} / \mathrm{D}$ converter output values into engineering units in the host controller. Hence, these values are read from the module at the first stage of operation. These calibration values are implemented in most input modules, for example NI 9201, 
9215, and 9221. In order to use this calibration, the LabVIEW FPGA provides a node for each port consisting of an LSB, an offset and a nominal range of each specific module [118] which can be used to calculate the calibrated engineering unit.

In the next step, the FPGA performs signal capturing directly from the NI 9201 and low-pass filtering before the signals are sent through a DMA (Direct Memory Access) FIFO loop. It should be noted from Figure 4.6 that analog input NI 9201 does not have a built-in anti-aliasing filter to prevent unwanted signals. Therefore, a careful design will be discussed to determine a proper low-pass filtering development in the next section.

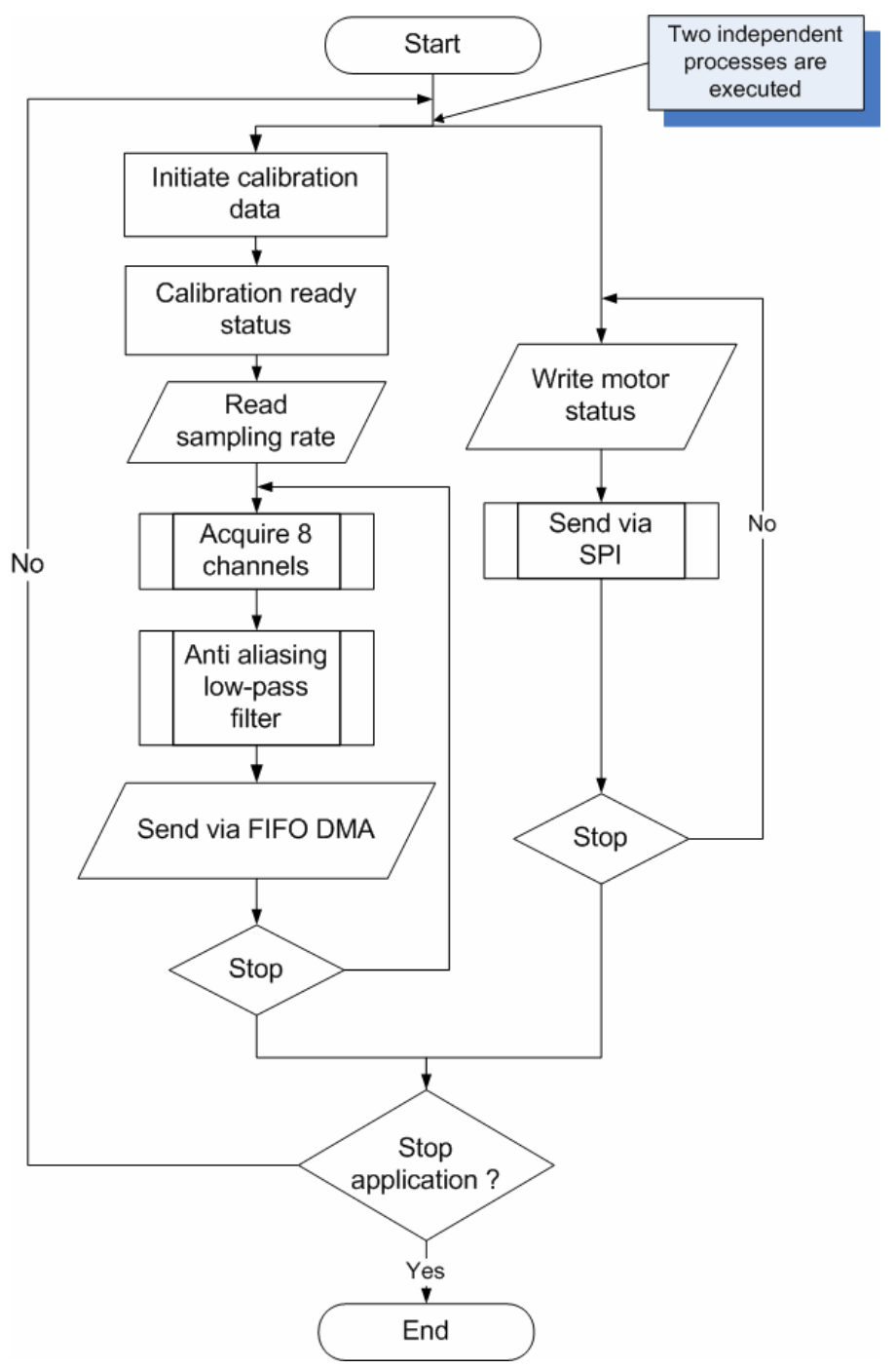

Figure 4.13 FPGA flowchart

Another branch of the FPGA flowchart involves the writing the motor status through the SPI bus to the indicator module mentioned in Section 4.3. The writing process basically implements the SPI triggering technique, based on the timing 
diagram of the NLSF595. Both developments will be discussed further in Sections 4.4.1 and 4.4.2.

Figure 4.13 also shows data synchronization between the FPGA and host controller, done using DMA FIFO. This technique is one of the integration techniques of the CompactRIO system besides polling and interrupts. These techniques, including the implementation of the DMA, will be discussed briefly in the Section 4.4.3.

Finally, the flowchart shown in figure above is designed based on A LabVIEW FPGA that is converted into an FPGA implementation through several simple steps. This overview will be explained in Section 4.4.4.

\subsubsection{Digital Low-pass Filtering Development}

Digital low pass filtering was chosen in this design to remove unwanted high frequencies during fault frequency analysis of the induction motor signals. This digital filter over-samples the input signals captured by the CompactRIO analog input module. In this research, the IIR filter was chosen because it usually executes faster, needs fewer coefficients, and does not require more memory than the FIR filter [119]. In addition, the fault frequency analysis does not need a linear phase response, which is one of the disadvantages of the IIR filter. However, the recursive nature of the IIR filter makes it more difficult to design than the FIR filter. Therefore, simulation and evaluation are carried out for this thesis.

Among the IIR filter types, the Butterworth filter was chosen to be applied in this design. Reasons for the implementation of the low pass Butterworth filter include no ripple in either passband and stopband, and it has a smooth monotonically decreasing frequency response in the transition region [119]. In this research, frequency response variation due to ripple effect in the passband is avoided because the magnitude of the fault frequencies are important to indicate the fault severity level in an induction motor.

In order to meet the requirements for condition monitoring, the floating-point Butterworth low pass filter was designed following the specifications given in Table 4.3 below. The low pass filter uses the maximum fault frequency base band of 1000 $\mathrm{Hz}$ as concluded previously in Chapter 2. The low pass filter also has a transition band around $50 \mathrm{~Hz}$ and, and ratio of the input to output signals in the passband of close to unity. In addition, the sampling frequency of low pass filter was chosen as $2200 \mathrm{~Hz}$. 
The filter analysis was carried out using the LabVIEW Digital Filter Toolkit ${ }^{\mathrm{TM}}$. The results are presented in Figure 4.14. This figure also shows that the low pass filter, which has an order of 16 , performs a relatively sharp roll-off frequency with a cut-off frequency around $1010 \mathrm{~Hz}$ at $-3 \mathrm{db}$.

Table 4.3 Low pass floating-point Butterworth filter specification

\begin{tabular}{|l|l|}
\hline \multicolumn{1}{|c|}{ Setting } & \multicolumn{1}{c|}{ Frequency } \\
\hline Passband edge frequency & $1000 \mathrm{~Hz}$ \\
\hline Stopband edge frequency & $1050 \mathrm{~Hz}$ \\
\hline Sampling frequency & $2200 \mathrm{~Hz}$ \\
\hline Stopband attenuation & $80 \mathrm{db}$ \\
\hline Passband ripple & $0.01 \mathrm{db}$ \\
\hline
\end{tabular}

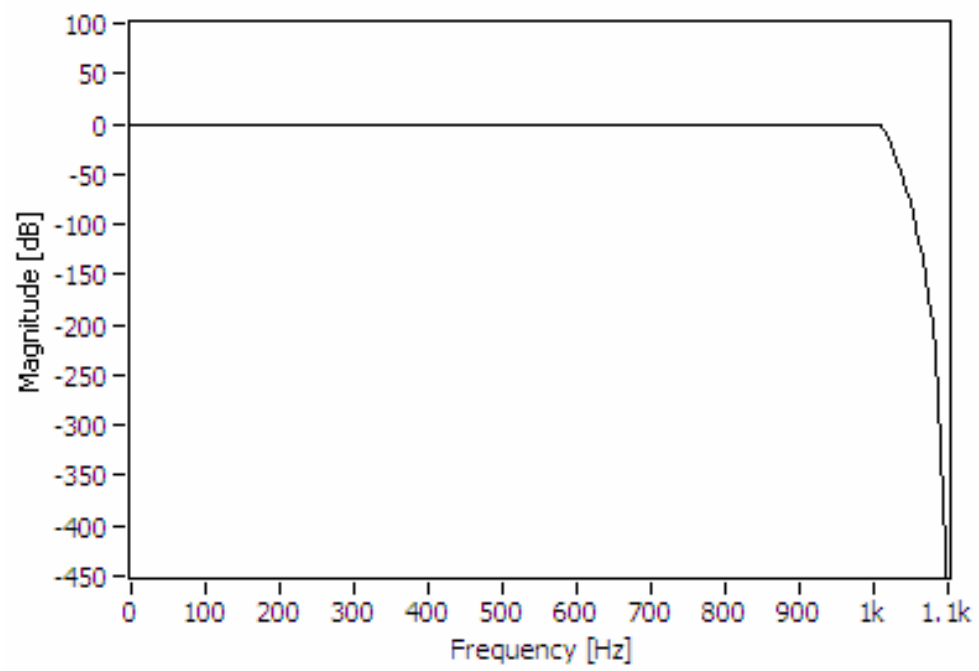

(a)

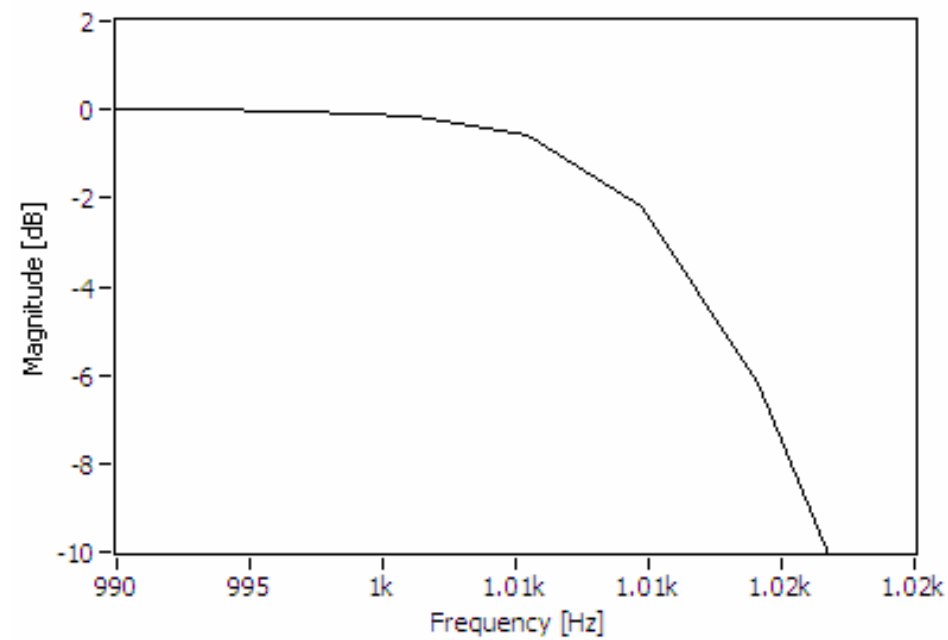

(b)

Figure 4.14. (a) Simulation results of the low pass filter design and (b) the zoom frequency response around the edge of the pass band showing the cut-off frequency 
Figure 4.15 shows another analysis to determine the characteristics of the lowpass filter. The top figure shows that all the poles in the pole-zero plot lie inside the unit circle, indicating the stability of the low pass filter. The bottom figure indicates the fast response of the filter, shown by a fast rise time. As can be seen in this figure, the low pass filter still has some overshoots which indicates the possibility of some amplitude distortion of the signals. The overshoot, which is around $30 \%$, is still be reasonable for a low pass filter implementation, however, further improvement to reduce the overshoot by implementing another configuration might need to be considered in the future.

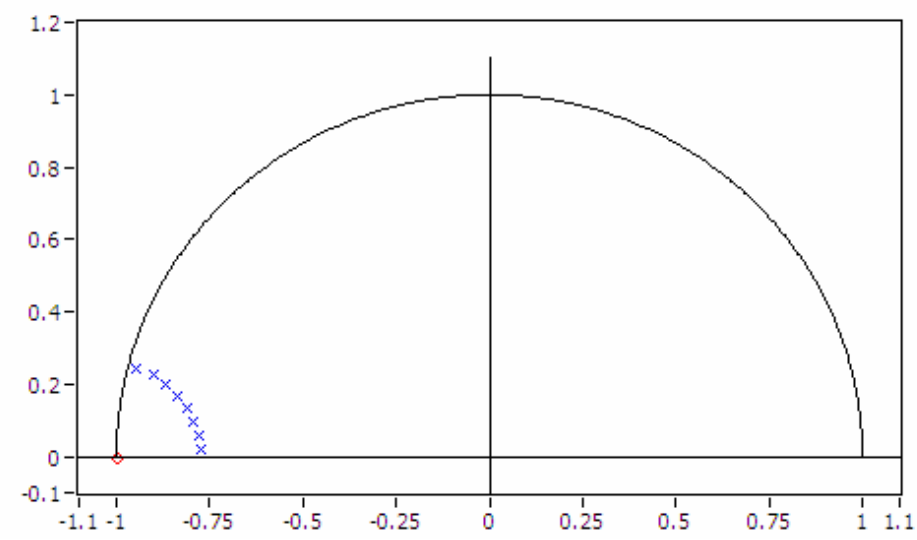

(a)

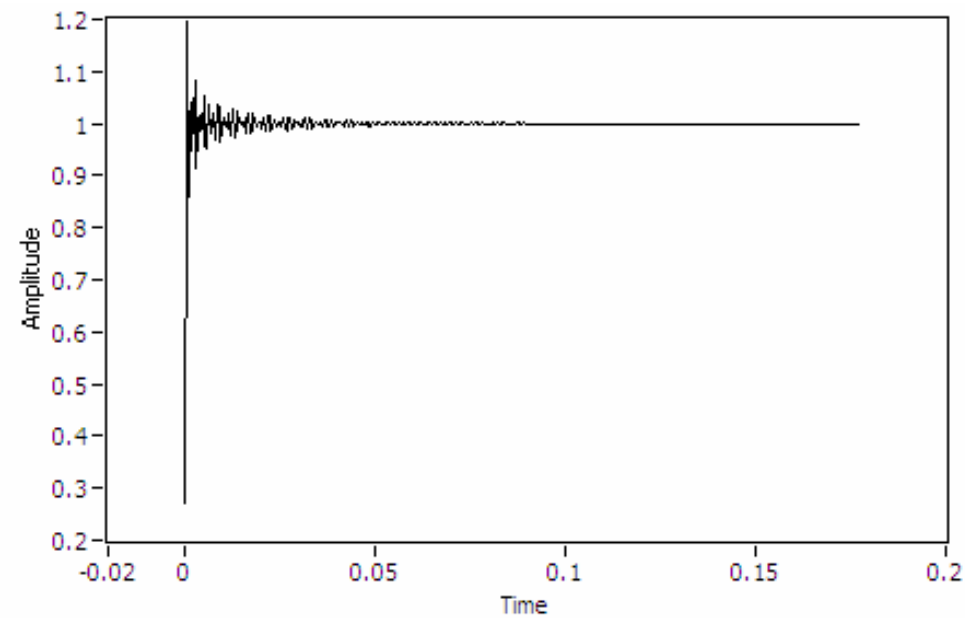

(b)

Figure 4.15 (a) Calculated pole-zero plots and (b) calculated step response of the low pass filter

In order to understand the frequency response, a simulation of the frequency response to a random signal input is carried out. Figures 4.16(a), and 4.16(b) show the frequency spectra of a random signal input and the corresponding low pass filtering output. These figures point out the performance of the low pass filter cutting off the 
frequency at more than $1000 \mathrm{~Hz}$, while frequencies below this are maintained at the same magnitude. The filter has a degree of attenuation above $1050 \mathrm{~Hz}$.

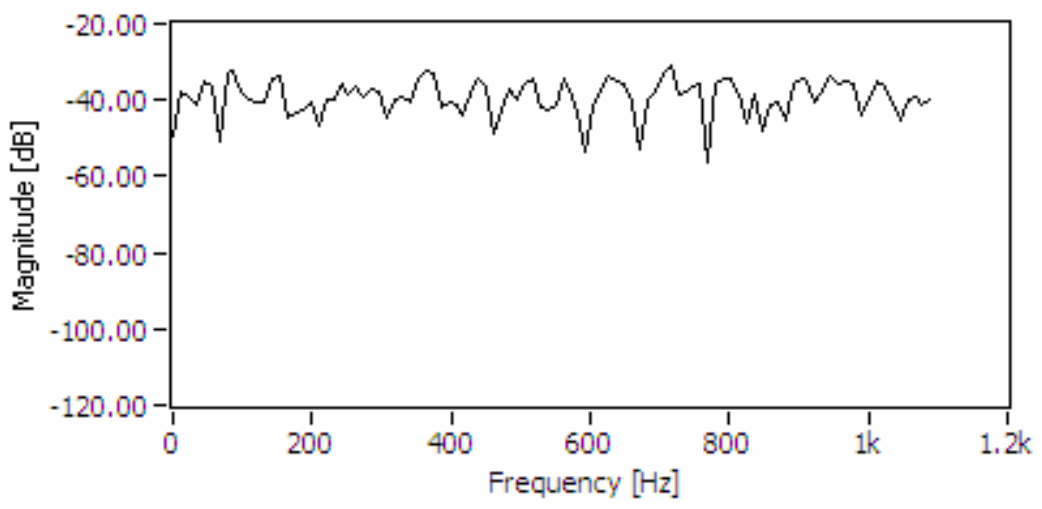

(a)

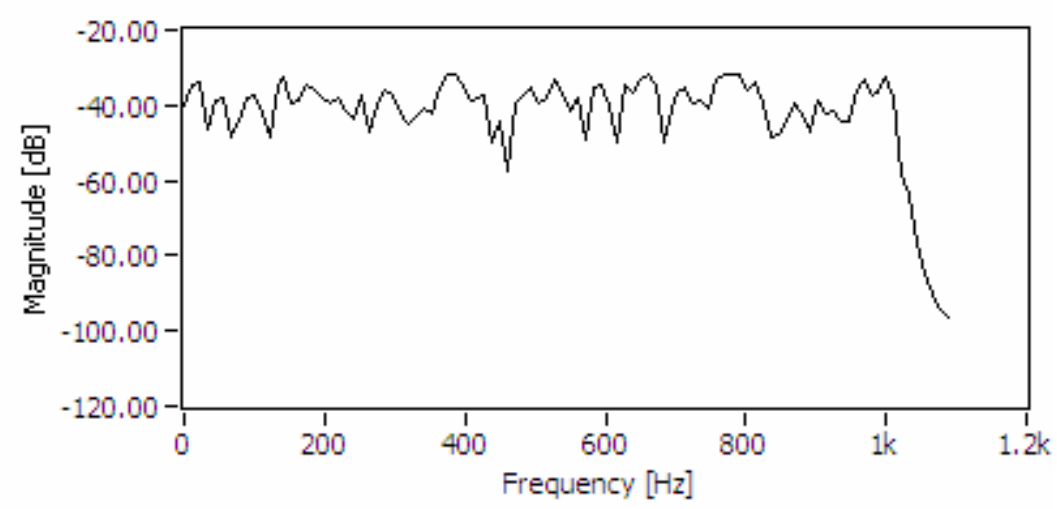

(b)

Figure 4.16 (a) Frequency spectra of simulation input random signals and (b) frequency spectra after low pass floating-point Butterworth filter

In the second step, the parameters obtained from previous analysis were implemented in a fixed-point FPGA filter that consumes less power and resources than the floating-point filter. Similar to the previous analysis, this step was undertaken using a LabVIEW Digital Filter Toolkit. The fixed-point filter must be set up by adjusting parameters to accommodate the finite precision constraints of the target specification platform through modeling and simulation.

Two parameters were determined in this step: the target structure and the coefficients of the quantification setting. These parameters determine the behaviour of the filter coefficients when the filter arithmetically processes the input signals. These parameters are important in fixed-point implementation. Unlike the floating-point filter where these parameters could be not significant, the different structures or coefficients of a fixed-point filter may in many cases result in different outputs [120]. 
Table 4.4 IIR low pass Butterworth filter fixed-point structure setting

\begin{tabular}{|l|l|}
\hline \multicolumn{1}{|c|}{ Setting } & \multicolumn{1}{c|}{ Type of setting } \\
\hline Target structure & $\begin{array}{l}\text { IIR Cascade Second-Order Section Form II } \\
\text { Transposed }\end{array}$ \\
\hline Scale before quantization & Time domain 1-Norm \\
\hline $\begin{array}{l}\text { Coefficient quantization } a / k \\
\text { and } b / v \text { word length }\end{array}$ & 16 bit \\
\hline
\end{tabular}

Table 4.4 shows the parameters determined for the fixed-point low pass filter. IIR Cascade Second-Order Section Form II was chosen because it requires less memory for saving internal states compared with Direct Transform I and II [120]. Furthermore, this type of filter is not sensitive to the effect of finite word length, which may be an issue with the Direct Form I and II implementations [120]. The word length indicates the number of bits that are used to represent a fixed-point number. Compared with the IIR Cascade Second-Order Section Form II, the transposed structure of this form offers better execution time because of its additional temporary storages during the summation operation [120].

Table 4.4 explains the scaling of coefficients that can be determined before quantization of coefficients to ensure that all accumulators in this filter are using the same data range. Four types of scaling can be performed [120]:

- no-normalization

- time domain 1-Norm (ensures dynamic range within specific range but reduces signal to rounding noise ratio)

- time domain 2-Norm (has high signal to rounding noise ratio but does not guarantee dynamic range output value), and

- time domain Inf-Norm (does not guarantee dynamic range output value).

Both 1-Norm and 2-Norm bring advantages which are needed at the same time for the condition monitoring system. In this research, Time Domain 1-Norm was chosen as guaranteeing the same data range. 


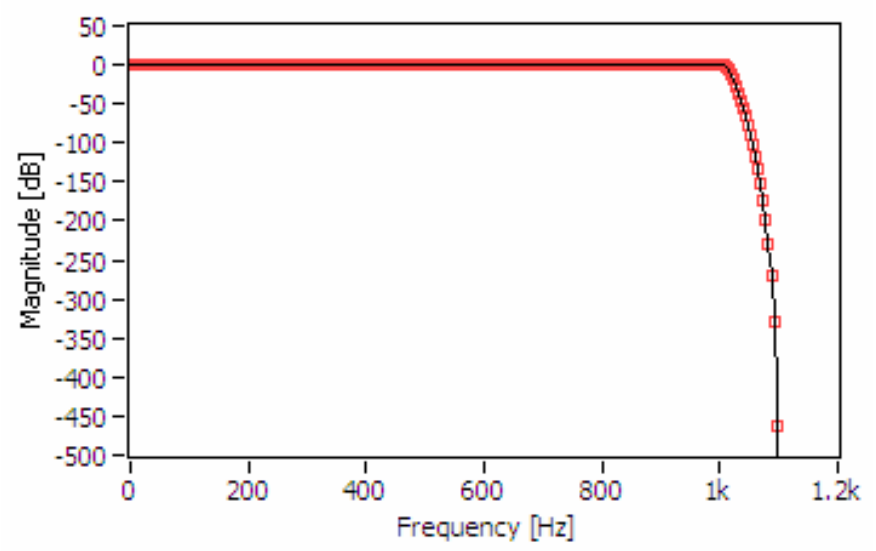

Figure 4.17 Comparison of floating-point and fixed-point frequency response

The comparison of the frequency response of this fixed-point structure, which is based on the previous floating-point filter, can be seen in Figure 4.17. Based on 16bit word length coefficients (see Table 4.4), both quantized fixed-point coefficients, 24 forward coefficients, and 16 reverse values show no under-or-over values compared with the floating-point references. This comparison as illustrated in Figure 4.17, also shows that the fixed-point filter (red marks) closely meets with the floatingpoint reference (black line). This result indicates that the frequency response of a fixed-point filter using these parameters is similar to the floating-point reference. Because of this, the performance of the fixed-point filter might be similar to the performance of floating-point filter.

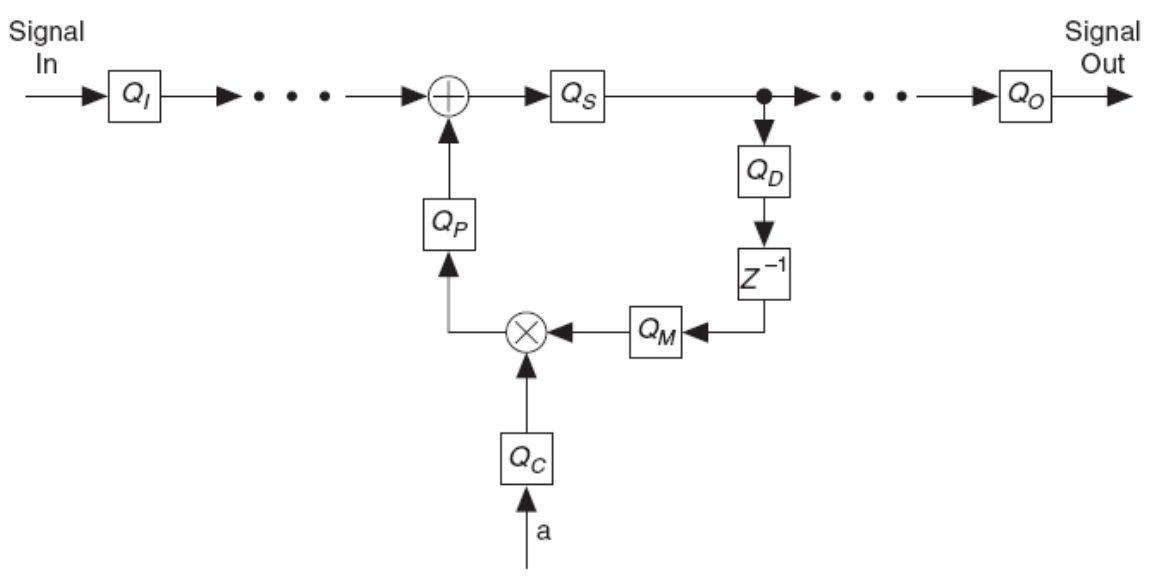

Figure 4.18 A fixed-point model of low pass filter which has the following quantizers :

- $Q_{I}$ is input quantizer for the input signal of a fixed-point filter

- $Q_{S}$ is sum quantizer for the summation of a fixed-point adder

- $Q_{D}$ is delay quantizer for the input of a delay element

- $Q_{M}$ is multiplicand quantizer for the multiplicand of a fixed-point multiplier 
- $Q_{C}$ is filter coefficients quantizer for the reference floating-point filter (can be coefficients $a / k$ or coefficients $b / v$ )

- $Q_{P}$ is product quantizer for the product of a fixed-point multiplier

- $Q_{O}$ is output quantizer for the output signal of a fixed-point filter

In the third step, after quantizing the coefficients of the filter, intermediate operands and results are also need to be determined for fixed-point filter. Figure 4.18 shows an example of quantizers in a fixed-point filter. This modeling has been conducted through trial and error by considering the effect of the coefficient quantization after all quantizers were simulated. Several settings have been established following several trials as presented in Table 4.5

Table 4.5 IIR low pass Butterworth filter fixed-point modeling coefficients

\begin{tabular}{|c|c|c|c|c|}
\hline \multirow{2}{*}{ Quantizers } & \multicolumn{4}{|c|}{ Type of setting } \\
\cline { 2 - 5 } & Word length & $\begin{array}{c}\text { Integer word } \\
\text { length }\end{array}$ & $\begin{array}{c}\text { Overflow } \\
\text { mode }\end{array}$ & $\begin{array}{c}\text { Round-off } \\
\text { mode }\end{array}$ \\
\hline Input & 16 & 1 & saturation & nearest \\
\hline Output & 16 & 3 & wrap & truncation \\
\hline Multiplicand & 32 & 2 & wrap & truncation \\
\hline Product & 32 & 2 & wrap & truncation \\
\hline Sum & 32 & 2 & wrap & truncation \\
\hline Delay & 32 & 2 & wrap & truncation \\
\hline
\end{tabular}

In Table 4.5, the word length indicates the number of bit to represent total fixed-point numbers. In addition, integer word length illustrates the integer part of a fixed-point number including the signed bit. As listed in Table 4.5, the long word length values were selected to minimize the effect of numerical errors caused by the fixed-point representation. However, it should be considered that this long word length will increase the hardware resources required.

The overflow mode setting of Table 4.5 represents how to handle overflow and underflow of each quantizer. There are two types of overflow and underflow handler: saturation and wrap mode. A quantizer in saturation mode will convert specific numbers to the maximum representable numbers in the case of overflow and to the minimum representable numbers in the case of underflow. In the wrap mode, a quantizer will change a value from minimum to maximum in the case of underflow and vice versa. Saturation mode is difficult and complex to be implemented in LabVIEW FPGA platform, even though it can avoid signal discontinuities and abrupt 
changes in amplitude. Therefore, the wrap mode was selected in this design for the most quantizers.

The final column is about the round-off mode that determines the quantizer rounding a specific fixed-point number. All quantizers, except for the input quantizer, employed a truncation mode to reduce the complexity of implementation. The nearest mode is implemented in the input quantizers to maintain real value as closesly as possible before going through other quantizers.

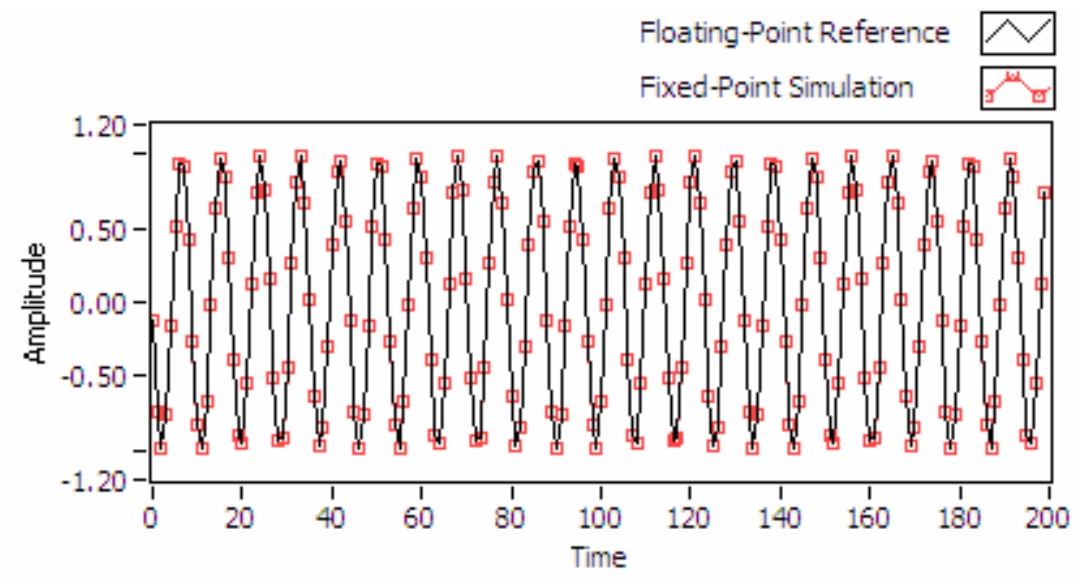

Figure 4.19 Comparison of floating-point and fixed-point simulation result of $200 \mathrm{~Hz}$ sinusoid input signal

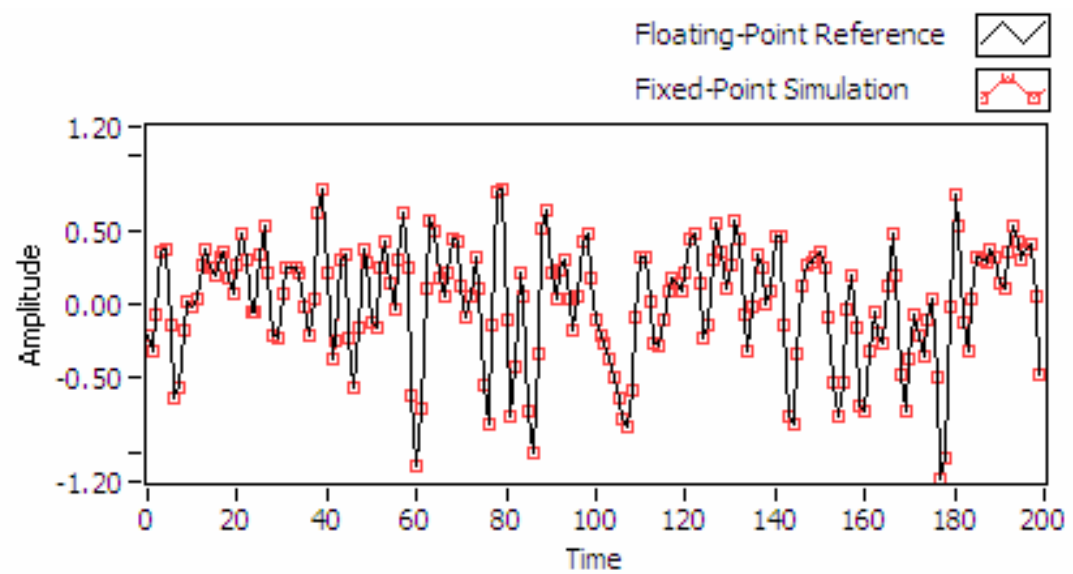

Figure 4.20 Comparison of floating-point and fixed-point simulation result of random input signal

Figures 4.19 and 4.20 show the comparison of a filtered sinusoid signal and random signal. It can be seen that the fixed-point filter is able to resemble characteristics of the floating-point reference filter, whatever the input signals. These results also point out that fixed-point filter performance is sufficiently similar to the 
reference without any underflows or overflows in each quantizer, as can be seen in Table 4.6.

Table 4.6 Statistic simulation of each quantizer

\begin{tabular}{|c|c|c|c|c|c|}
\hline Parameter & Limits & $\begin{array}{c}\text { Max/Min Actual } \\
\text { Value }\end{array}$ & $\begin{array}{c}\text { Over } \\
\text { flows }\end{array}$ & $\begin{array}{c}\text { Under } \\
\text { flows }\end{array}$ & Operations \\
\hline Input & $+1.999 /-1.999$ & $+0.999 /-0.969$ & 0 & 0 & 9000 \\
\hline Output & $+7.999 /-7.999$ & $+1.582 /-1.558$ & 0 & 0 & 9000 \\
\hline Multiplicand & $+3.999 /-3.999$ & $+0.999 /-0.969$ & 0 & 0 & 144000 \\
\hline Product & $+3.999 /-3.999$ & $+1.179 /-1.186$ & 0 & 0 & 369000 \\
\hline Sum & $+3.999 /-3.999$ & $+1.179 /-1.189$ & 0 & 0 & 882000 \\
\hline Delay & $+3.999 /-3.999$ & $+0.406 /-0.405$ & 0 & 0 & 144000 \\
\hline
\end{tabular}

In the final step, considering that all parameters and coefficients have been examined previously, the IIR Butterworth filter was developed. The VI of the IIR low pass filter module is shown in Figure 4.21. The IIR Butterworth filter is capable of handling eight channels of data acquisition signals, with maximum sampling frequency $178.57 \mathrm{kHz}$. This value was produced by a VI of LabVIEW FPGA code generator. This value illustrates the maximum input sampling frequency of each channel that generated FPGA can process at $40 \mathrm{MHz}$ FPGA clock rate. In addition, this filter is also fitted with one FPGA memory and two multiplications subVIs, as shown in Figure 4.21.

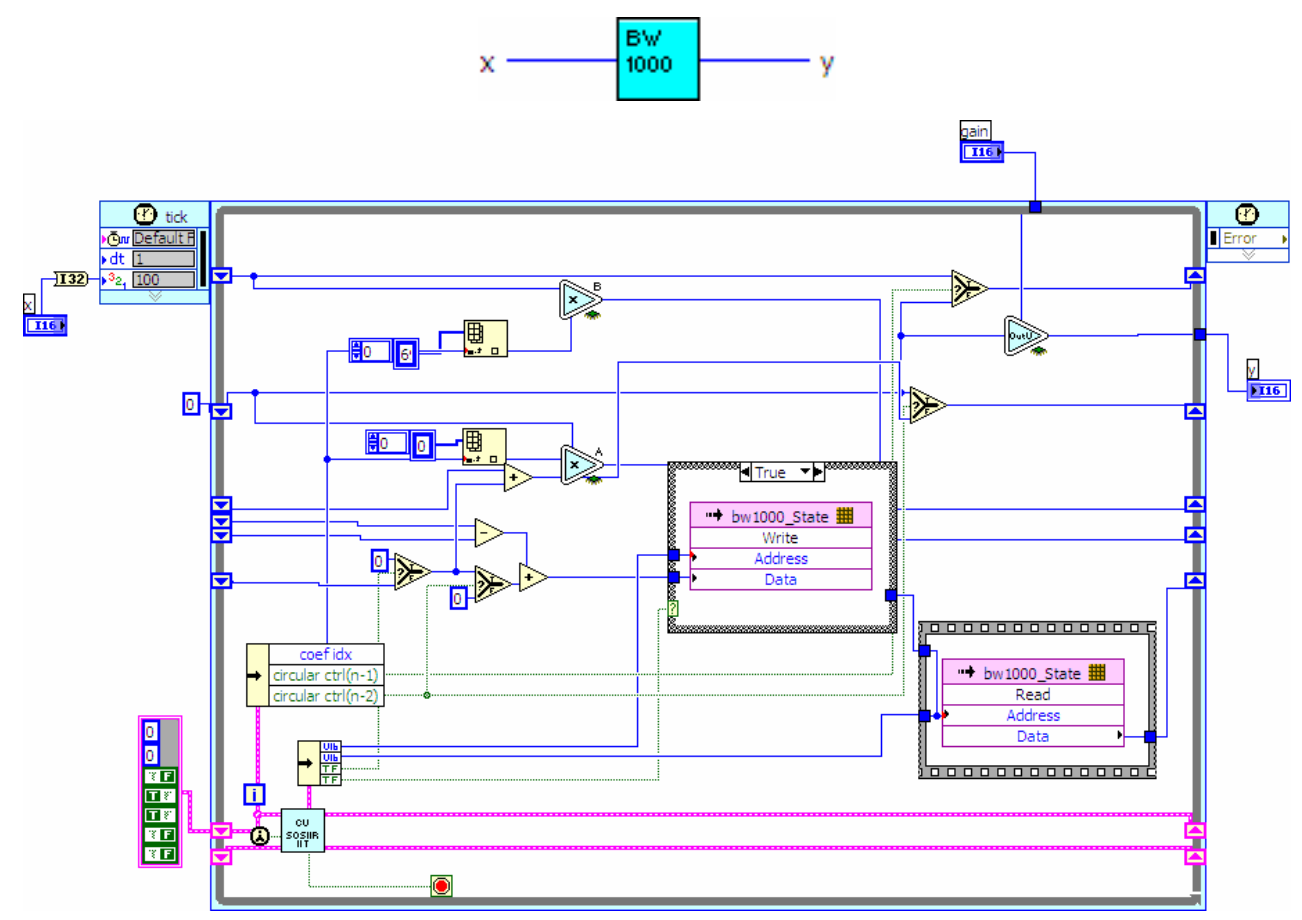

Figure 4.21 Low Pass Butterworth Filter icon VI (top), and block diagram of the VI (bottom) 


\subsubsection{LED Driving Development}

As mentioned previously, the LED indicator module is driven by FPGA through SPI bus. The FPGA controls two NLSF595 LED drivers as shown in Table 4.7, and Figure 4.22, the timing diagram of the NLSF595 IC.

Table 4.7 Function table NLSF595 [115]

\begin{tabular}{|c|c|c|c|c|c|c|c|c|c|}
\hline \multirow[b]{2}{*}{ Operation } & \multicolumn{5}{|c|}{ Inputs } & \multicolumn{4}{|c|}{ Resulting Function } \\
\hline & $\begin{array}{l}\text { Reset } \\
\text { (SCLR) }\end{array}$ & $\begin{array}{l}\text { Serial } \\
\text { Input } \\
\text { (SI) }\end{array}$ & \begin{tabular}{|l|} 
Shift \\
Clock \\
(SCK)
\end{tabular} & $\begin{array}{l}\text { Reg } \\
\text { Clock } \\
\text { (RCK) }\end{array}$ & $\begin{array}{c}\text { Output } \\
\text { Enable } \\
\text { (OE) }\end{array}$ & $\begin{array}{c}\text { Shift } \\
\text { Register } \\
\text { Contents }\end{array}$ & $\begin{array}{l}\text { Storage } \\
\text { Register } \\
\text { Contents }\end{array}$ & $\begin{array}{l}\text { Serial } \\
\text { Output } \\
\text { (SQH) }\end{array}$ & \begin{tabular}{|c} 
Parallel \\
Outputs \\
(QA - QH)
\end{tabular} \\
\hline Clear shift register & $\mathrm{L}$ & $x$ & $x$ & $\mathrm{~L}, \mathrm{H}, \downarrow$ & L & L & $\mathrm{U}$ & L & $\mathrm{U}$ \\
\hline $\begin{array}{l}\text { Shift data into shift } \\
\text { register }\end{array}$ & $\mathrm{H}$ & $\mathrm{D}$ & $\uparrow$ & $L, H, \downarrow$ & $\bar{L}$ & $\begin{array}{c}\mathrm{D} \rightarrow \mathrm{SR}_{\mathrm{A}} \\
\mathrm{SR}_{\mathrm{N}} \rightarrow \mathrm{SR}_{\mathrm{N}+1}\end{array}$ & $\mathrm{U}$ & $\mathrm{SR}_{\mathrm{G}} \rightarrow \mathrm{SR}_{\mathrm{H}}$ & $\mathrm{U}$ \\
\hline $\begin{array}{l}\text { Registers remains } \\
\text { unchanged }\end{array}$ & $\mathrm{H}$ & $x$ & $\mathrm{~L}, \mathrm{H}, \downarrow$ & $x$ & L & $\mathrm{U}$ & ** & $\mathrm{U}$ & ** \\
\hline $\begin{array}{l}\text { Transfer shift register } \\
\text { contents to storage } \\
\text { register }\end{array}$ & $\mathrm{H}$ & $x$ & $\mathrm{~L}, \mathrm{H}, \downarrow$ & $\uparrow$ & $\bar{L}$ & $\mathrm{U}$ & $\mathrm{SR}_{\mathrm{N}} \rightarrow \mathrm{STR}_{\mathrm{N}}$ & * & $\mathrm{SR}_{\mathrm{N}}$ \\
\hline $\begin{array}{l}\text { Storage register remains } \\
\text { unchanged }\end{array}$ & $x$ & $x$ & $\mathrm{x}$ & $L, H, \downarrow$ & $\bar{L}$ & * & $\mathrm{U}$ & * & $\mathrm{U}$ \\
\hline Enable parallel outputs & $x$ & $x$ & $x$ & $x$ & $\bar{L}$ & * & 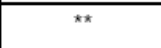 & * & Enabled \\
\hline $\begin{array}{l}\text { Force outputs into high } \\
\text { impedance state }\end{array}$ & $x$ & $x$ & $x$ & $x$ & $\mathrm{H}$ & * & $\star \star$ & * & $\bar{Z}$ \\
\hline
\end{tabular}

$\begin{array}{llll}S R=\text { shift register contents } & D=\text { data }(L, H) \text { logic level } & \downarrow=\text { High-to-Low } & * \text { * depends on Reset and Shift Clock inputs } \\ \text { STR = storage register contents } & U=\text { remains unchanged } & \uparrow=\text { Low-to-High } & * * \text { depends on Register Clock input }\end{array}$

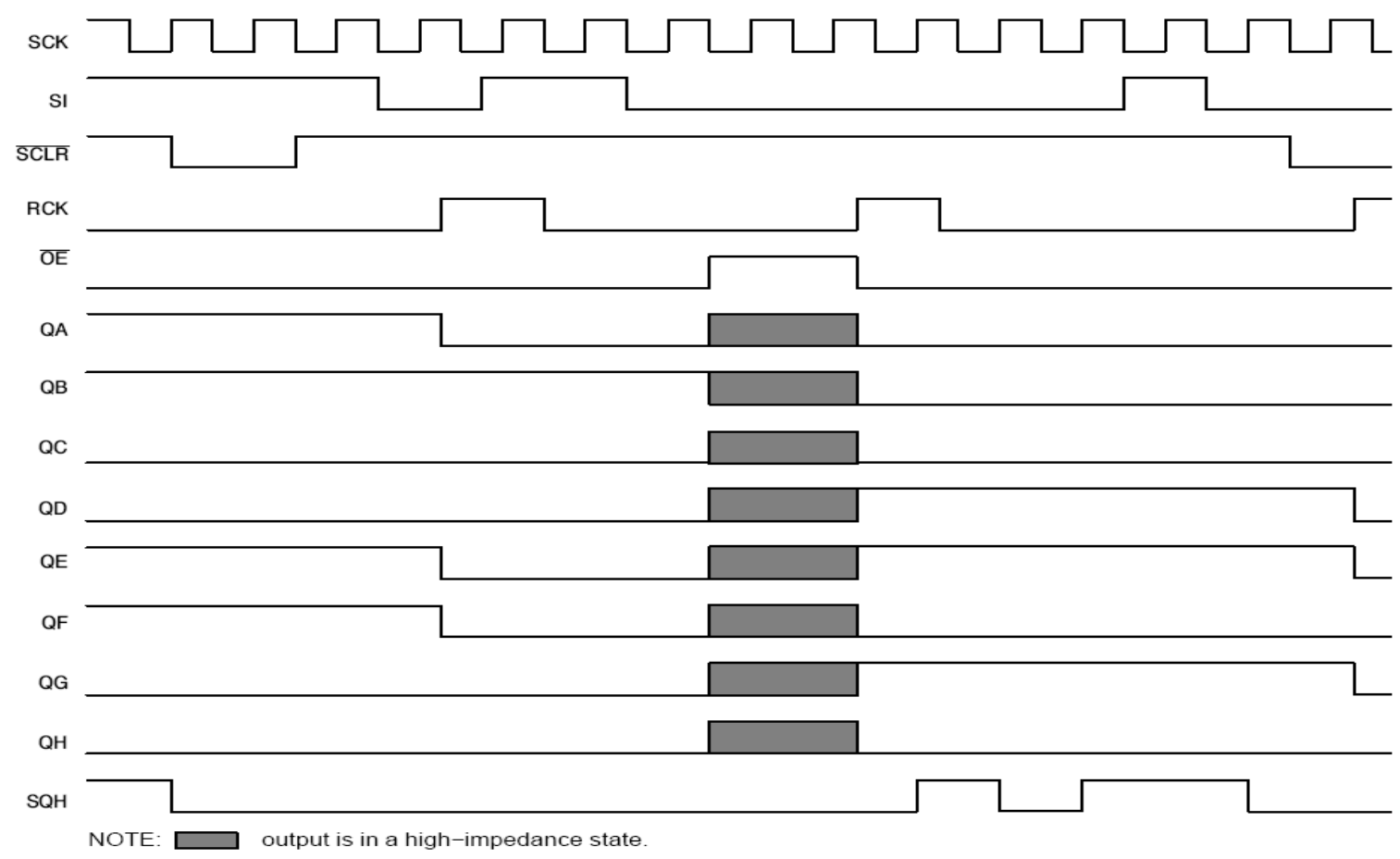

Figure 4.22 Timing diagram of NLSF 595 LED driver

As illustrated in the function table and timing diagram, three channels are important to drive the LED drivers: SCK, SI and RCK. The SCK clock signal is 
driven by SPI_CLK port of FPGA, SI data by MISO, and RCK by CS. In addition, SCLR and OE signals have been tied up high and low respectively. As shown in the timing diagram, the $\mathrm{n}$ data bits is passed in serial mode every clock signal to the first LED driver. For more than 8 data bits, another $\mathrm{n}$ bits data than is delivered from the first LED driver to the second LED driver through the SQH pin (see Figure 4.44). Data is transferred to the 8 channels output every time IC is enabled through CS. Figure 4.23 shows a flowchart of the FPGA to drive the LED indicator module, based on the logic mentioned previously.

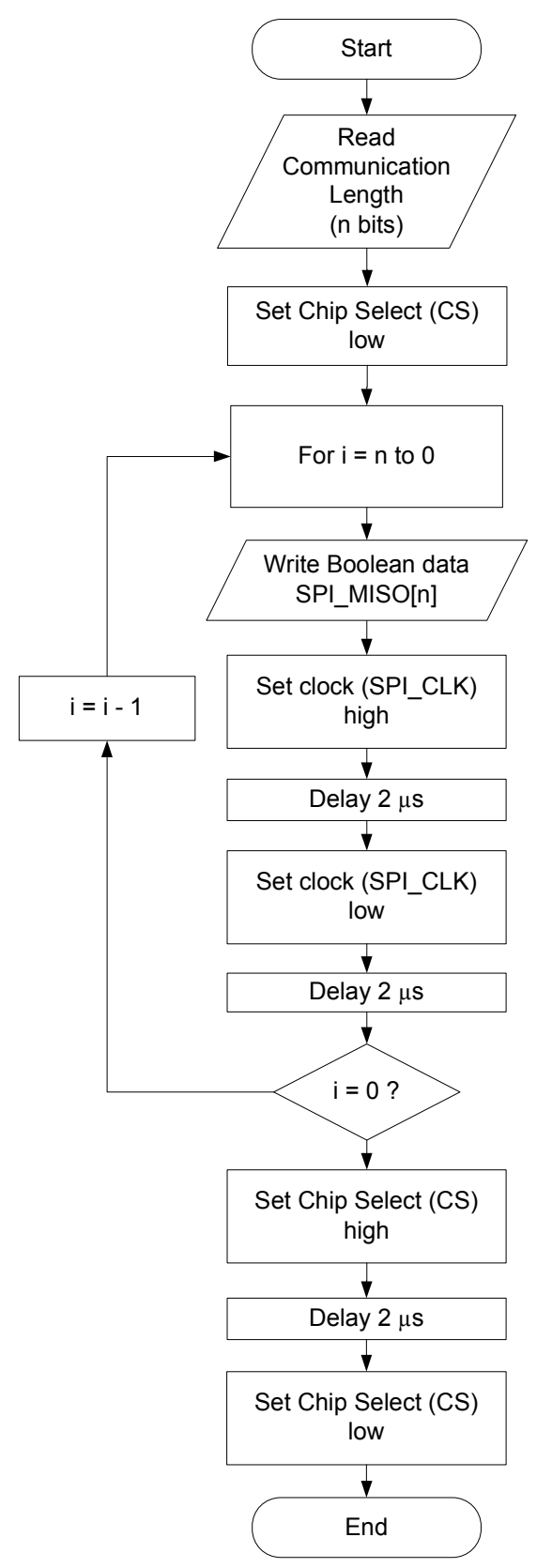

Figure 4.23 FPGA indicator module 


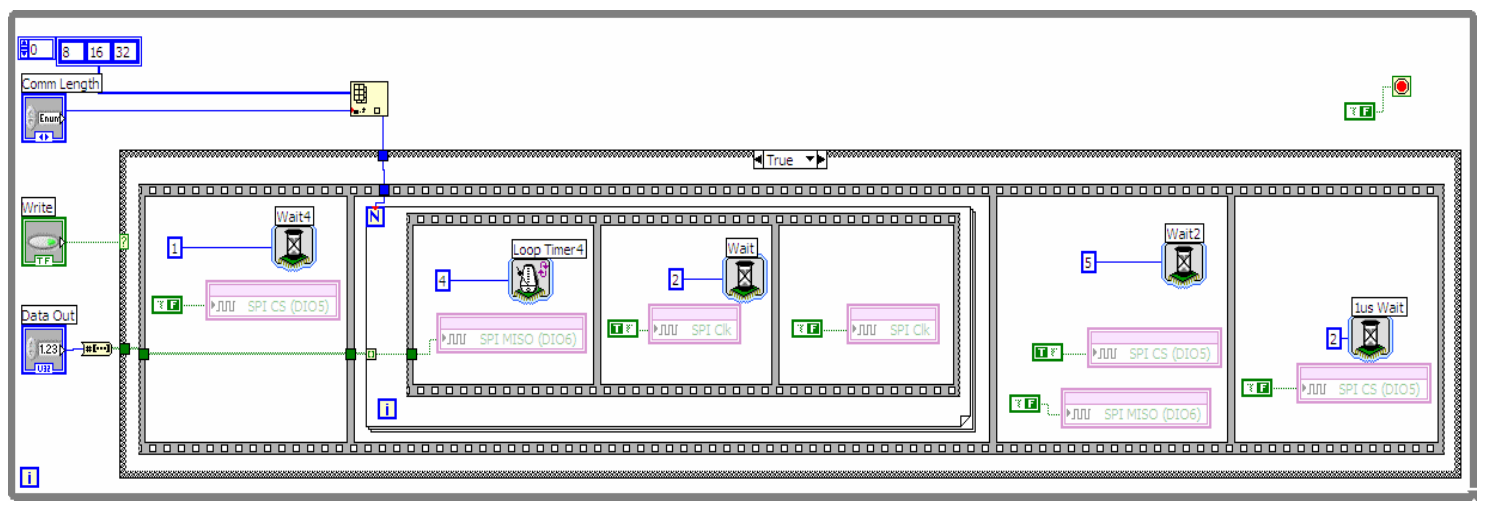

Figure 4.24 Block diagram VI of the indicator module

It can be seen that the FPGA will read the number of bits at the first step, followed by low chip select to clear any status or task running at that time in NLSF595. Then, a bit of an $n$ element of array data is written before a clock signal is generated. This step is repeated $n$ times according to the communication length. After all data is written, the chip select signal is enabled to power on the LEDs array. Detail of the block program is presented in Figure 4.24. It should be noted that the communication length can be set using 8,16 or 32 bit, depending on the number of NLSF595 utilized. In addition, the delay time and counter shown in this figure can be programmed in tick $(25 \mathrm{nSec}), \mathrm{nSec}$ or $\mathrm{mSec}$ units depending on the expected speed of execution of the indicator module.

\subsubsection{Compiling and Uploading FPGA}

This section will provide an overview of the technique for compiling and uploading a LabVIEW FPGA VI. Figure 4.25 shows these two processes which must be incorporated.

In the first step, as illustrated in the top figure, a graphic-based VI is converted into VHDL, known as an intermediate file. This file is then sent to the Xilinx ISE server to be compiled producing a bit file. This Xilinx compiler can be installed in the same machine, or on another remote machine. The bit file is than delivered back to the LabVIEW FPGA and associated with an FPGA VI. 


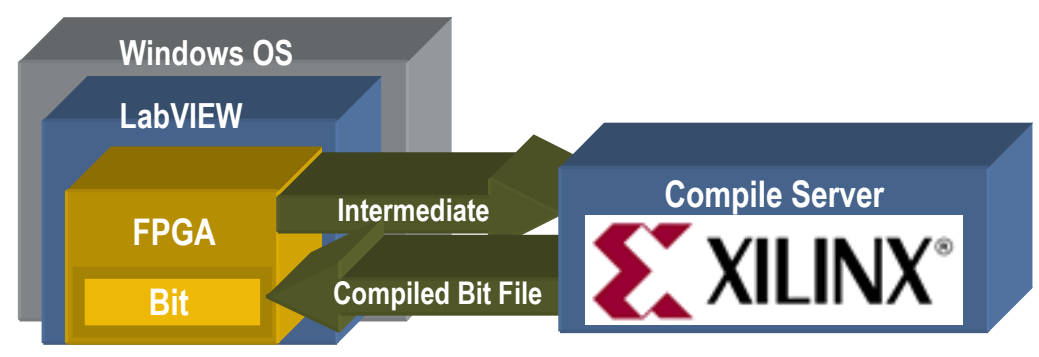

(a)

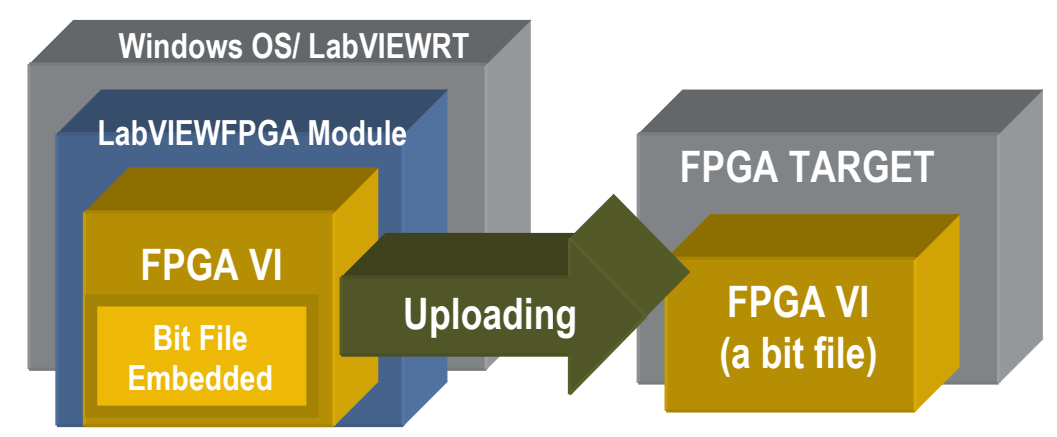

(b)

Figure 4.25 (a) Compiling process of an FPGA VI, and (b) uploading a bit file to the FPGA target [118]

In the next step, the compiled bitstream is uploaded to the FPGA target, as can be seen in Figure 4.25.b. This uploading can be done automatically every time a run button of the VI is initiated. With this technique, the front panel of the VI FPGA can be seen in the Windows development machine, while the FPGA VI is running in the target. In another technique, a bit file can be uploaded into a LabVIEW Real Time environment permanently as start-up software in a Real-time Host Controller. This technique enables a VI run automatically each time the CompactRIO is switched on, without an additional PC/development machine. This last technique is implemented in this research.

\subsection{Real-time Condition Monitoring Software Development}

As illustrated previously in Figure 4.5, the data analysis software is performed in the real-time host controller cRIO-9004. The monitoring software is basically divided into three main parts: host to FPGA synchronization, frequency analysis, and fault analysis. A detailed flowchart is shown in Figure 4.26. 


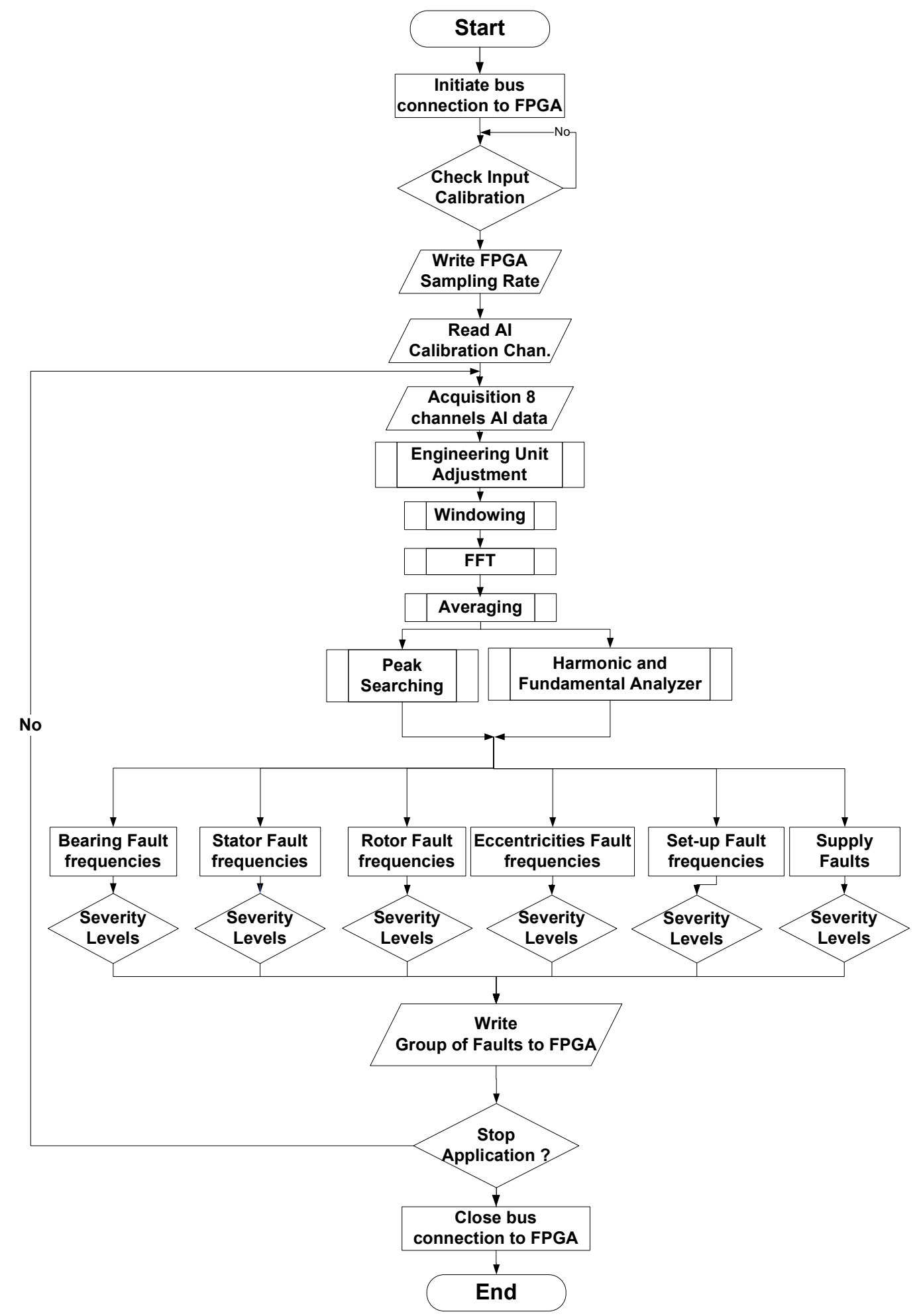

Figure 4.26 Flowchart of condition monitoring software in the real-time host controller

As can be seen in this figure, the initialization connection, and data calibration including engineering unit conversion of each sensor signal, are performed first. The analogue sensor signals are than captured, followed by an FFT analysis, frequency averaging, and fault frequency peak detection. Following the peak detection 
algorithm, a number of sub-routines are implemented to identify specific faults based on the fault frequency equations detailed in Chapter 3. Then, fault severity level algorithms are implemented to estimate the specific fault levels based on the amplitudes of the fault frequencies. The output of this section is utilized to drive the indicator module for the condition monitoring of the motor.

Details of this development will be discussed in the following sections. Section 4.5.1 will present an overview of the FPGA and host synchronization, including the data transfer implementation. Section 4.5 .2 will briefly discuss frequency analysis and the components employed. Finally, fault analysis including peak detection, severity level detection, and an interface to send data status of the motor to the FPGA will be covered.

\subsubsection{Host to FPGA Synchronization and Data Transfer}

As illustrated in Figure 4.6, the connection between the FPGA and real-time host controller is achieved through the PCI bus using specific protocols. In this section, the data transfer protocols will be briefly discussed, as well as the host to FPGA synchronization and implementation in the CompactRIO condition monitoring system.

Communication protocol is provided to synchronize communication between the FPGA chassis and the real-time host controller. Basically, the VIs in the FPGA and the VIs in the real-time controller are independent of each other, and so are inherently asynchronous. Therefore, there is a need to determine how to synchronize the data transmission and reception between these two modules to avoid losing any data. However, it should be noted that in this monitoring application, the synchronization used is not as tight as that used for timing control between the FPGA and host controller in real-time control applications.

Three synchronization modes can be used for data transfer: interrupt-based handshaking, DMA-based handshaking, traditional polling or a combination of these $[121,122]$. The interrupt-based mode synchronization can be efficient because it transfers data only when an interrupt is generated. It might alleviate the load on the host controller CPU, but it is not fast enough in certain applications compared with the traditional polling method. The DMA-based mode is much faster than other methods. This method sends data directly from the FPGA memory to real-time host memory, 
while requiring very little of processor resources through a FIFO technique. In this research, the DMA approach is preferred to increase the system operating speed.

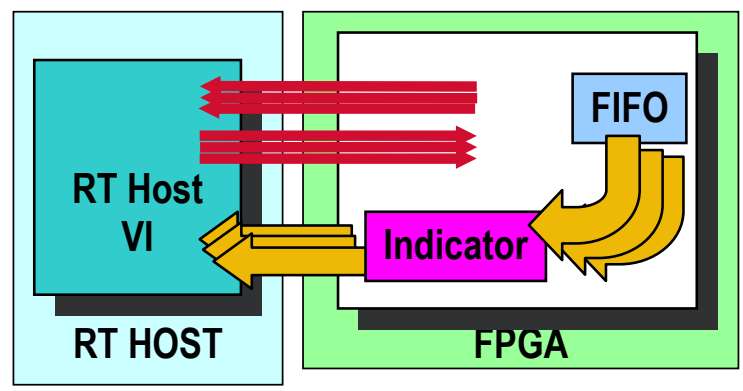

Figure 4.27 Basic data transfer and synchronization

Basic data transfer and synchronization using DMA are illustrated in Figure 4.27 [122]. After signals are captured in input, data is sent to the FIFO and placed in the front panel indicator. The FPGA then indicates the availability of data to a VI of the host application after completion of a block of data reading in FIFO. The host controller then reads one data item at a time, and sends back an acknowledgement signal afterwards. In response to this signal, the FPGA retrieves the next data point from FIFO to be placed in the indicator. This sequence occurs until all data are successfully transferred. Based on this DMA approach, there are three types of DMA data transfer techniques [122]:

1. Blocking : the host controller will read all data from FPGA once data are available, before expiring at certain time

2. Polling : the host controller will read subsequent data from FPGA that has been determined before, until all data are captured

3. Interrupt : the data will be read through DMA when an interrupt has signaled the data availability

Simple testing procedures were undertaken to gain an understanding of the characteristics of three types of DMA data transfer. In these tests, the DMA polling seems erratic for eight channel dynamic measurement. In the second case, even though the DMA interrupt is able to transfer a large number of data and requires less CPU overhead, the DMA interrupt is slowest compared with the others. Therefore, the DMA blocking is implemented with a modification as shown in Figure 4.28, where time of expiry is set indefinitely. Buffering is added during the data transfer to ease the real-time host processor. 


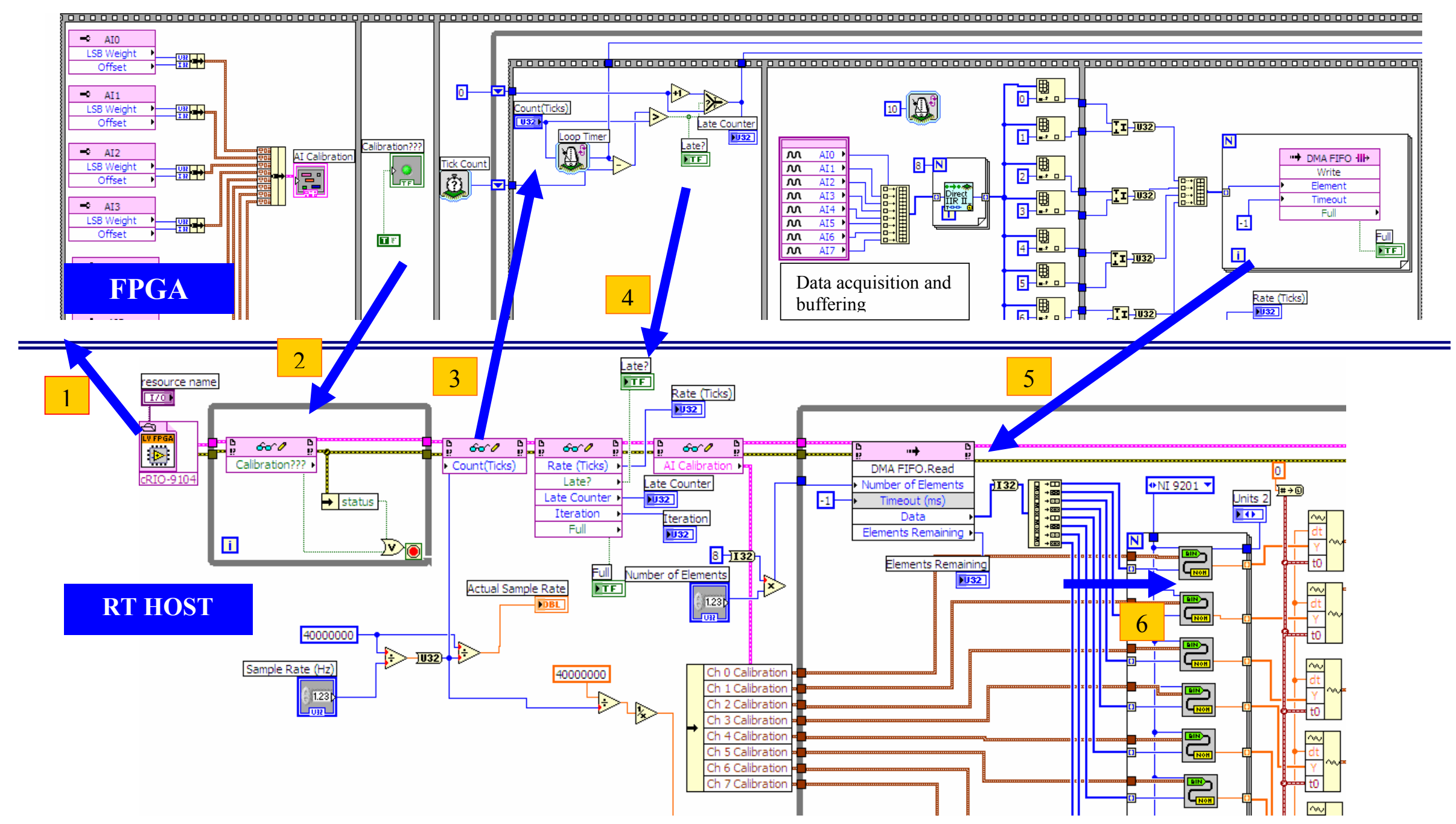

Figure 4.28 Data synchronization between CompactRIO FPGA and main program CompactRIO Real-Time Host 
Figure 4.28 illustrates FPGA and host synchronization between FPGA and the real-time host controller. This figure also details the flowchart of real-time software shown in Figure 4.26. As can be seen in this figure, the host synchronization is started by opening the FPGA VI Reference, as indicated by arrow 1. This opening routine will run existing compiled FPGA VI in the FPGA. In case the compiled FPGA VI does not exist in FPGA, the opening routine will download compiled FPGA VI to the FPGA. Then, FPGA calibration status is read, as indicated by arrow 2. After the calibration parameter returns a true value status, arrow 3 illustrates that the real-time host sends sampling-rate information to the FPGA. The FPGA will acquire data based on this sampling rate information after checking the status of any late counter indicated by arrow 4. In the next step, eight channel signals are acquired, buffered, and sent to a DMA engine in the FPGA. The DMA engine will send the captured signals through DMA FIFO, as shown by arrow 5. The captured signals in real-time host then are converted from binary values to equivalent nominal values, as pointed out by arrow 6 . These nominal values of waveform signals are ready to be converted into frequencies. This will be discussed in the following sections.

\subsubsection{Frequency Analysis}

Frequency domain transformation, which basically employs FFT analysis, is carried out after pre-processing tasks, and followed by other signal processing functions. As illustrated in Figure 4.26, these pre-processing tasks include engineering unit adjustments and windowing. After FFT, frequency averaging is done. Table 4.8 shows the function, setting available, and setting chosen for each algorithm of the signal processing for the CompactRIO condition monitoring system, including the FFT.

Table 4.8 LabVIEW VI functions used for frequency analysis [123, 124]

\begin{tabular}{|l|l|l|}
\hline Algorithms & Functions & Setting Available \\
\hline $\begin{array}{l}\text { Engineering } \\
\text { Unit }\end{array}$ & $\begin{array}{l}\text { Scaling signals from voltage } \\
\text { to specific engineering units } \\
\text { Adjustment } \\
\text { according to the sensitivity } \\
\text { of each sensor }\end{array}$ & $\begin{array}{l}\text { - Sensor sensitivity setting } \\
\text { - Engineering unit label (custom and } \\
\text { built-in labels : } \\
\text { V,A,m/s } / \mathrm{m} / \mathrm{s}, \mathrm{mm} / \mathrm{s}, \mathrm{Pa}, \mathrm{N}, \mathrm{g}, \mathrm{mm} / \mathrm{s} \text { ) }\end{array}$ \\
\hline $\begin{array}{l}\text { Smoothing } \\
\text { Windowing }\end{array}$ & $\begin{array}{l}\text { Improving spectral } \\
\text { characteristics, such as } \\
\text { reducing spectral leakage } \\
\text { and separating two very } \\
\text { close frequencies }\end{array}$ & $\begin{array}{l}\text { Wide range of windowing } \\
\text { (Hanning, Kaiser, Hamming, }\end{array}$ \\
& $\begin{array}{l}\text { Blackman-Harris, Blackman, Exact } \\
\text { Blackman, Flat-Top, Gaussian, } \\
\text { Dolph-Chebyshev) }\end{array}$ \\
\hline
\end{tabular}


Table 4.9 LabVIEW VI functions used for frequency analysis [123, 124] (continued)

\begin{tabular}{|l|l|l|}
\hline Algorithms & Functions & Setting Available \\
\hline FFT & $\begin{array}{l}\text { Measuring frequency content } \\
\text { of sampled signals }\end{array}$ & $\begin{array}{l}\bullet \text { Number of FFT Lines frequencies } \\
(512,1024,2048,4096) \\
\text { - Y-axis view (db or linear) } \\
\text { - Bandwidth }\end{array}$ \\
\hline Averaging & $\begin{array}{l}\text { Improving accuracy through } \\
\text { several successive frequency } \\
\text { averagings }\end{array}$ & $\begin{array}{l}\text { Averaging mode (vector, RMS, } \\
\text { peak hold) }\end{array}$ \\
& & $\begin{array}{l}\text { Weighting mode (linear or } \\
\text { exponential) } \\
\text { - Number of average }\end{array}$ \\
\hline
\end{tabular}

It should be noted that LabVIEW has provided all these functions. Several functions are also available in a complete LabVIEW Professional Development ${ }^{\mathrm{TM}}$ and LabVIEW Sound and Vibration Toolkit ${ }^{\mathrm{TM}}$. The engineering adjustment unit is provided in a single sub VI as illustrated in Figure 4.29(a) In addition, three algorithms implemented in this research, i.e. smoothing windowing, FFT, and averaging, are developed in a single package for the optimum application in LabVIEW Sound and Vibration Toolkit ${ }^{\mathrm{TM}}$, as shown in Figure 4.29(b).

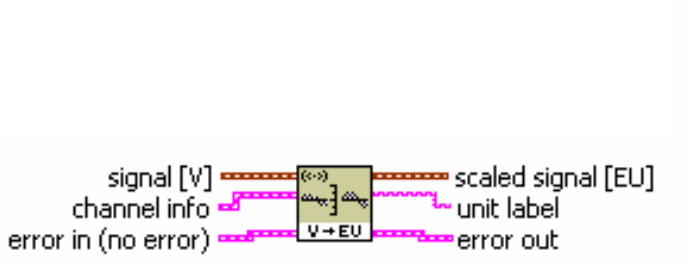

(a)

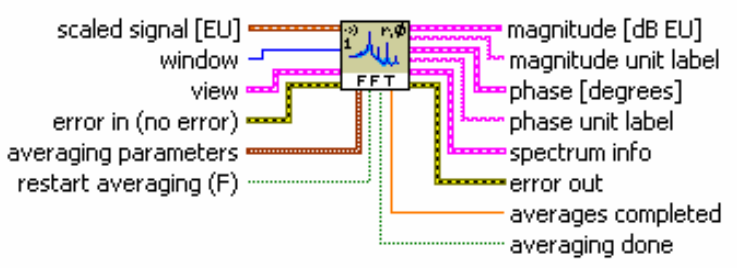

(b)

Figure 4.29 Sub VI of frequency analysis : (a) A sub VI of scaling voltage to Specific Engineering Unit (SVL Scale Voltage to EU) and (b) A sub VI of computing FFT spectrum (SVFA FFT Spectrum (Mag-Phase))

The VI of engineering unit adjustment in Figure 4.29(a) will scale signal from volt into the selected engineering unit (EU) for $\mathrm{N}$ channel input signals. The VI is able to scale each channel according to its own channel information. The Channel Info connector shown in this figure contains information about the measurement system used before the data acquisition system, including sensor sensitivity, engineering unit, $\mathrm{dB}$ reference value, and weighting filter. These features allow amplitude of each input signal to be normalized during the FFT.

Amplitude of stator current, flux leakage and vibration signals in $\mathrm{dB}$ can be normalised manually to obtain accurate fault analysis. In the case of stator current and 
flux leakage, amplitude input signals are normalized to the amplitude of fundamental line frequency $50 \mathrm{~Hz}$. It was found that the amplitude of fundamental frequency $50 \mathrm{~Hz}$ was $14 \mathrm{~dB}$ or equal with $5.012 \mathrm{~V}$. Using 5.012 as the $\mathrm{dB}$ reference in this $\mathrm{VI}$, the peak of fundamental frequency was shifted to $0 \mathrm{~dB}$ through equation $\mathrm{dB}=20 \log _{10}\left(A / A_{r}\right)$ in the FFT sub VI, where $A$ is the measured amplitude, $A_{r}$ is the reference amplitude. Consequently, this normalization can show the sideband frequencies clearly. In the case of vibration monitoring, the sensor sensitivity in $\mathrm{mV} / \mathrm{g}$ can be introduced. Hence, the amplitude for severity level analysis can be in positive value and standardized with ISO 2372, 10816 or 7919. However, in this research, the sensor sensitivity adjustment for vibration monitoring is not implemented to avoid complex variation of the baseline threshold of peak searching VI for eight channel inputs.

The sampled stationary signals may have several discontinuities. These discontinuities may exist especially when input signals are not sampled at an integer number of cycles. These noninteger cycle frequency components of the signal will not correspond exactly to one of spectrum frequency lines. These frequencies are spread over adjacent frequency lines and known as spectral leakage.

As explained in Table 4.8, smoothing windows minimize spectral leakage caused by the discontinuities of truncated waveforms of a sampled signal [124]. The smoothing window operates similar to the narrowband low pass filters to reduce the amplitude of the discontinuities [124]. The spectrum of windowed signals is produced in this sub VI by convolving the spectrum of the original signal with the spectrum of the smoothing window. The result of the smoothing window will change the shape of the signal in the time domain and frequency domain. Each smoothing window will produce specific shapes of windowed signals suitable for specific application. For example, a window that balances between amplitude accuracy and spectral resolution, such as the Blackman-Harris window, is required for the fault frequency detection and severity level analysis application. Further analysis of the smoothing window selection will be discussed in Chapter 5.

The FFT in sub VI illustrated in Figure 4.29(b), measures the frequency content of the windowed signals. The FFT generates the average frequency content of a signal over the total acquisition. In [124], an FFT can also be illustrated to a set of parallel filters of bandwidth $\Delta f$ centred at each frequency increment from DC to $\left(F_{S} / 2\right)$ - $\left(F_{S} / N\right)$, where $F_{S}$ is the sampling frequency and $N$ is the number of samples. It 
should be noted that this VI computes FFT in a real-valued signal, and returns magnitude in $\mathrm{db}$ and phase in degree at the sampling rate $2200 \mathrm{~Hz}$. In addition, for eight channels, the maximum number of samples of each channel is only 4096. This is because the analog input module can perform only limited binary values for 12 bit. The frequency produced after this FFT is then transferred for the further step of the frequency analysis known as averaging.

To improve measurement accuracy, successive frequency averaging can be performed in this FFT VI. There are three types of frequency averaging available: vector averaging, RMS averaging, and peak hold [124]. RMS averaging reduces signal fluctuations but not the noise floor spectrum. Unlike RMS averaging, vector averaging will eliminate noise floor by averaging the real part of FFT spectrum separately. The peak hold retains the peak levels of the averaged quantities. In addition, during the frequency averaging, each new spectral record can be weighted when RMS or vector averaging is performed using either linear or exponential weighting. For linear weighting, several spectral records are combined with equal weighting. However, for exponential weighting, new spectral data is emphasized more than old data, and is a continuous process. In this research, five times RMS averaging with linear weighting is chosen. The reason for this is that five times of averages with linear weighting may smooth noise floor, while the actual amplitude of peak frequency can be still retained. All parameters and selection of the frequency analysis are summarized in Table 4.10.

Table 4.10 Summary of frequency analysis of the real-time condition monitoring software

\begin{tabular}{|l|l|}
\hline Parameter & Value \\
\hline Sampling rate & $2000 \mathrm{~Hz}$ \\
\hline Analog to digital (A/D) resolution & $12 \mathrm{bit}$ \\
\hline Nyquist frequency & $1000 \mathrm{~Hz}$ \\
\hline Number of samples & 4096 \\
\hline Frequency resolution & $0.244 \mathrm{~Hz}$ \\
\hline Y axis view type & $\mathrm{dB}$ \\
\hline Window & Blackman-Harris \\
\hline Averaging & $\begin{array}{l}\text { Mode: RMS averaging } \\
\text { Weighting mode: Linear } \\
\text { Number of average: 5 }\end{array}$ \\
\hline
\end{tabular}

In summary, that each LabVIEW function covers almost everything needed for signal processing of the condition monitoring software. Each function has a few key 
selectable parameters, which may be adjusted according to the measurement conducted. These parameters can be selected to gain the most acceptable monitoring results. Indeed, it could be beneficial if further studies compared the effectiveness of each combination for the best results in condition monitoring.

\subsubsection{Fault Frequency Analysis}

Fault analysis is conducted according to the fault frequencies discussed in Chapter 3. These predictors are established as a comparator with peaks of frequency, which have been detected after several algorithms mentioned in the previous section. These predictors are programmed into simple sub VIs. These custom written LabVIEW VIs are presented below, including the built-in limit testing VI of LabVIEW.

Table 4.11 Custom and built-in VIs implemented for fault frequency analysis

\begin{tabular}{|c|c|}
\hline Bearing Faults & Descriptions \\
\hline $\begin{array}{c}\text { Ball Diameter } \\
\text { Number of Balls }\end{array} \quad \begin{array}{c}\text { Ball Defect Frequency } \\
\text { Outer Race Frequency } \\
\text { Bearing Pitch Diameter } \\
\text { Contact Angle of the Balls } \\
\text { Inner Race Frequency } \\
\text { Outer Race Frequency-Approx... } \\
\text { Motor fundamental frequency }\end{array}$ & $\begin{array}{l}\text { Bearing fault frequencies } \\
\text { (see Equations } 3.5 \text { to } 3.8 \text { ) }\end{array}$ \\
\hline Bearing Faults & Descriptions \\
\hline $\begin{array}{c}\text { Slip } \\
\text { Number of Balls } \\
\text { detected fundamental freque... }\end{array}$, & $\begin{array}{l}\text { Bearing faults (see } \\
\text { Equation } 3.9 \text { to } 3.12 \text { ) }\end{array}$ \\
\hline $\begin{array}{l}\text { Ball Diameter } \\
\text { Number of Balls } \\
\text { Bearing Pitch Diameter } \\
\text { detected fundamental freque... } \\
\text { Contact Angle of the Balls } \\
\text { detected fundamental Line } \mathrm{f} . . .\end{array}$ & $\begin{array}{l}\text { Bearing related faults } \\
\text { with stator current, } \\
\text { voltage and flux leakage } \\
\text { (see Equation } 3.13 \text { ) }\end{array}$ \\
\hline \multicolumn{2}{|l|}{ Stator Faults } \\
\hline $\begin{array}{l}\text { Detected Frequencies } \\
\text { harmonic components } \\
\text { detected fundamental freque... } \\
\text { detected fundamental amplit... }\end{array}$ & $\begin{array}{l}\text { Stator faults with } \\
\text { vibration monitoring } \\
\text { (see. Figure } 3.10 \text { ) }\end{array}$ \\
\hline 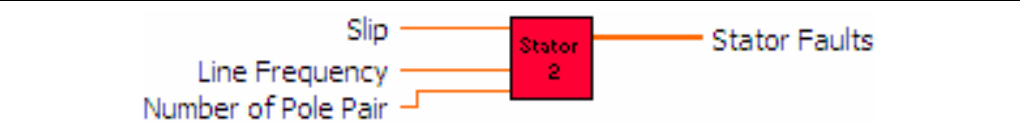 & $\begin{array}{l}\text { Stator faults (see } \\
\text { Equation } 3.14 \text { ) }\end{array}$ \\
\hline \multicolumn{2}{|l|}{ Rotor Faults } \\
\hline 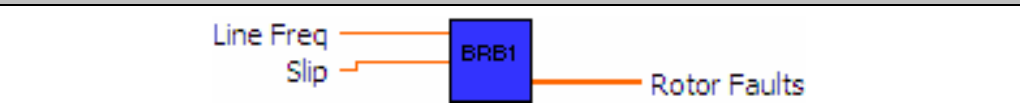 & $\begin{array}{l}\text { Broken Rotor Bars (see } \\
\text { Equation 3.16) }\end{array}$ \\
\hline
\end{tabular}


Table 4.11 Custom and built-in VIs implemented for fault frequency analysis (continued)

\begin{tabular}{|c|c|c|}
\hline \multicolumn{3}{|l|}{ Rotor Faults } \\
\hline $\begin{array}{c}\text { Number of pole Pair } \\
\text { Llipe Frequency }\end{array}$ & Rotor Faults & $\begin{array}{l}\text { Broken Rotor Bars (see } \\
\text { Equation } 3.17 \text { ) }\end{array}$ \\
\hline $\begin{array}{c}\text { Slip } \\
\text { detected fundamental freque... } \\
\text { Number of Pole Pair }\end{array}$ & Rotor & $\begin{array}{l}\text { Broken Rotor Bars (see } \\
\text { Equation } 3.15 \text { ) }\end{array}$ \\
\hline $\begin{array}{l}\text { harmonic components } \\
\text { Detected Fundamental Freque... } \\
\text { Detected Fundamental Freque... } \\
\text { pole }\end{array}$ & Rotor & $\begin{array}{l}\text { Broken rotor bar, bad } \\
\text { joint and shorted } \\
\text { lamination using } \\
\text { vibration } \\
\text { (see Figure } 3.11 \text { ) }\end{array}$ \\
\hline $\begin{array}{c}\text { Number of Rotor Bar } \\
\text { detected fundamental freque... } \\
\text { Line frequency }\end{array}$ & Rotor Faults & $\begin{array}{l}\text { Loose Rotor } \\
\text { (see Equation 3.19) }\end{array}$ \\
\hline \multicolumn{3}{|l|}{ Eccentricity Faults } \\
\hline $\begin{array}{l}\text { Slip } \\
\text { Line frequency } \\
\text { Number of Rotor Bar } \\
\text { Number of Pole Pair }\end{array}$ & Eccentricities & $\begin{array}{l}\text { Static Eccentricity } \\
\text { (see Equation } 3.20 \text { ) }\end{array}$ \\
\hline $\begin{array}{l}\text { Number of Pole Pair } \\
\text { Line Frequency } \\
\text { Number of Rotor Bar }\end{array}$ & Eccentricities & $\begin{array}{l}\text { Dynamic Eccentricity } \\
\text { (see Equation 3.20) }\end{array}$ \\
\hline $\begin{aligned} & \text { Line Frequency } \\
& \text { detected fundamental freque... }\end{aligned} \quad\left[\begin{array}{l}\mathrm{ECC} \\
\mathrm{MIX}\end{array}\right.$ & Eccentricities Mix & $\begin{array}{l}\text { Eccentricity with } \\
\text { vibration } \\
\text { (see Figure } 3.14 \text { ) }\end{array}$ \\
\hline Eccentricity Faults & & Descriptions \\
\hline \begin{tabular}{c|c|} 
Slip Frequency & Ecc \\
detected fundamental line f... & \\
Number of Pole Pairs & \\
\end{tabular} & - Eccentricities Mix & $\begin{array}{l}\text { Mix eccentricity } \\
\text { (see Equation 3.22) }\end{array}$ \\
\hline \multicolumn{3}{|l|}{ Set-up Faults } \\
\hline $\begin{array}{cc}\text { harmonic components } \\
\text { Detected Rotor Fundamental ... }\end{array}$ & Set-up related faults & $\begin{array}{l}\text { Misalignment } \\
\text { Mechanical Unbalance } \\
\text { Mechanical Looseness } \\
\text { (see Section 3.2.5) }\end{array}$ \\
\hline \multicolumn{3}{|l|}{ Calculated Slip frequency } \\
\hline $\begin{array}{l}\text { Line Frequency } \\
\text { Number of Pole Pair } \\
\text { Fundamental Frequency Rotor }\end{array}$ & SLIP & Calculates Slip frequency \\
\hline \multicolumn{3}{|l|}{ Limit Testing } \\
\hline $\begin{array}{c}\text { reset } \\
\text { Specification Cluster } \\
\text { error in (no error) }\end{array}$ & Limit & $\begin{array}{l}\text { Creates a limit as defined } \\
\text { by the input array X and } \\
\mathrm{Y} \text { (built-in LabVIEW VI) }\end{array}$ \\
\hline
\end{tabular}


Table 4.11 Custom and built-in VIs implemented for fault frequency analysis (continued)

\begin{tabular}{|c|c|}
\hline \multicolumn{2}{|l|}{ Limit Testing } \\
\hline 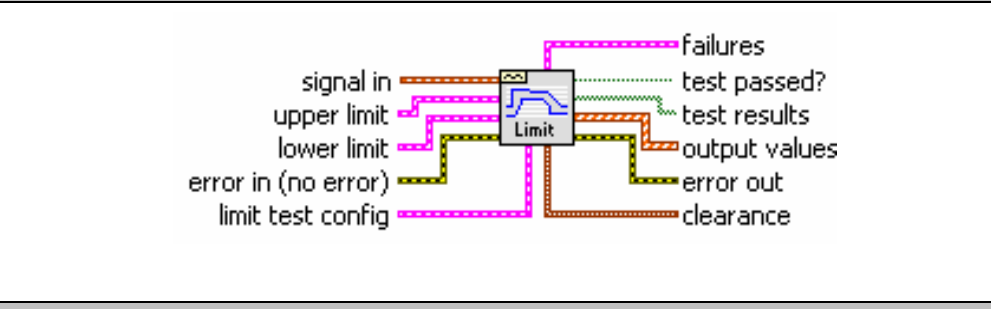 & $\begin{array}{l}\text { Performs limit testing on } \\
\text { cluster input data : } \\
\text { compares signal with } \\
\text { upper limit and lower } \\
\text { limit. (built-in LabVIEW } \\
\text { VI) }\end{array}$ \\
\hline \multicolumn{2}{|l|}{ Peak Searching and Fundamental Detection } \\
\hline $\begin{array}{l}\text { spectra } \\
\text { peak search settings } \\
\text { spectrum info } \\
\text { error in (no error) }\end{array}$ & $\begin{array}{l}\text { Performs peak search on } \\
\text { spectrum or spectra, } \\
\text { Single/multiple peaks }\end{array}$ \\
\hline 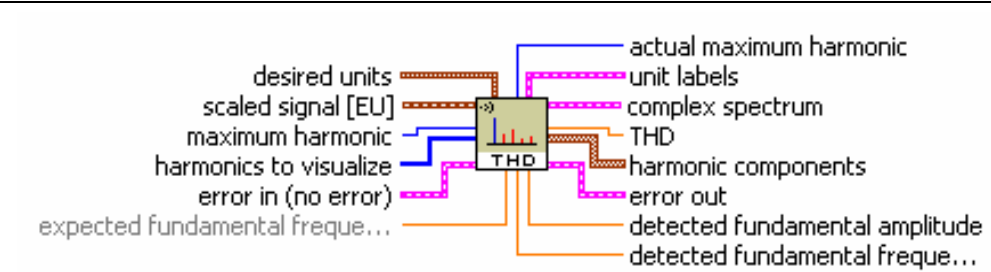 & $\begin{array}{l}\text { Detects fundamental and } \\
\text { harmonics frequency } \\
\text { including total harmonic } \\
\text { distortion }(T H D)\end{array}$ \\
\hline
\end{tabular}

As shown in Table 4.11 above, each sub VI implements an equation or a frequency pattern. Each sub VI is used as a fault frequency predictor through specific monitoring techniques. The frequency produced can be examined under a certain threshold for simple diagnosis. Each VI is part of the fault analysis and diagnosis section in the CompactRIO real-time monitoring software. Hence, the characteristics of each VI, and how the VIs work, needs to be discussed.

The following sections will consider several important issues. The first section will present how fundamental frequency and slip calculation is carried out. In the second section, several essential sub VIs of the fault frequency predictors will be detailed. The VIs are selected considering the most developed algorithm or technique of the fault frequency predictors. This selection also considers how the experimental evaluation of fault analysis can be done in a laboratory. These experimental evaluations are eccentricity related faults, broken rotor bars, shorted turn stator faults, and set-up related faults. Following several studies, examples of frequency predictors of these faults are also given including selected harmonic frequencies. The third section will discuss limit testing VI as the diagnosis tool. 


\subsubsection{Fundamental Frequencies and Slip Calculation}

Fundamental frequencies are detected through a fundamental and harmonic analyzer task, as shown in Figure 4.26. The task is carried out by a built-in LabVIEW sub VI, illustrated in Figure 4.30. The sub VI returns harmonic components, detected fundamental frequency, and total harmonic distortion (THD). The sub VI can be used not only to identify fundamental rotor speed $\left(F_{R}\right)$ but also fundamental line frequency $\left(F_{L}\right)$. In the case of the fundamental frequency not being the highest amplitude, an expected fundamental frequency can be introduced as a prediction value for the VI. This VI also returns harmonic components of these fundamental frequencies, both the amplitudes and the frequencies less than the Nyquist frequency. These amplitudes and frequencies are important for further analysis such as speed calculation, slip calculation, or severity level analysis.

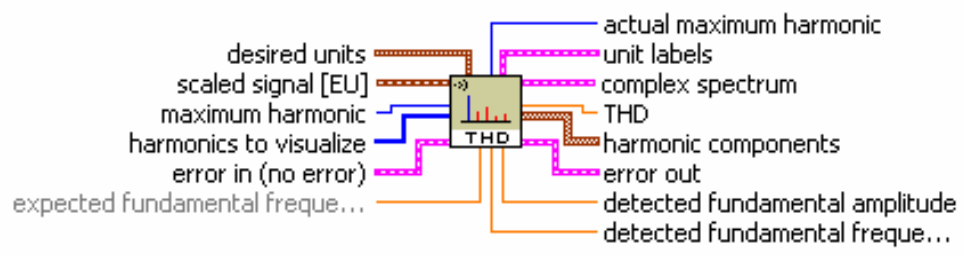

\section{Figure 4.30 A sub VI of harmonic analysis (SVT THD and Harmonics Components)}

In terms of speed calculation, two types of speed should be determined. These are synchronous speed or the speed of the rotating magnetic field $\left(N_{S}\right)$, and rotor speed, or mechanical rotational speed of the rotor $\left(N_{R}\right)$. Synchronous speed $\left(N_{S}\right)$ is calculated from the fundamental line frequency $\left(F_{L}\right)$ as indicated in Equation 3.1. Another speed, rotor speed $\left(N_{R}\right)$ is determined based on the fundamental rotor frequency $\left(F_{R}\right)$ as given in Equation 3.4. For this research, $F_{R}$ is identified from vibration monitoring signals and $F_{L}$ from stator voltage monitoring signals. It may be considered that fundamental frequency of vibration monitoring may not be the best parameter to determined $F_{R}$. This can be difficult, especially for a healthy motor where $F_{R}$ is sometimes low and overwhelmed by noise. Furthermore, the expected fundamental frequency introduced for the VI may not be accurate for load variation. This means that the predictor should be varied according to the variation of load. Hence, for further research, it would be better if the slip prediction was carried out directly using a dedicated rotor speed sensor or slip. 
Both synchronous and rotor speeds are employed to determine slip ( $s)$ as given in Equation 3.2 using a sub VI shown in Figure 4.31. It should be noted that slip can be identified directly from slip frequency at low frequency range (less than $10 \mathrm{~Hz}$ ) using flux leakage monitoring [51]. However, this technique needs very highresolution frequency (40000 data points, $0.01 \mathrm{~Hz}$ resolution) [51], which cannot be implemented using the current analog input module of the CompactRIO system. Hence, the slip is calculated based on synchronous and rotor speeds.

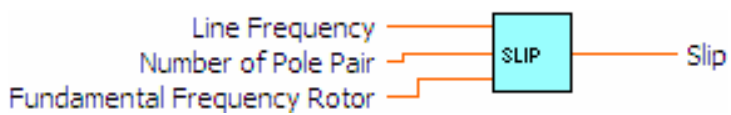

(a)

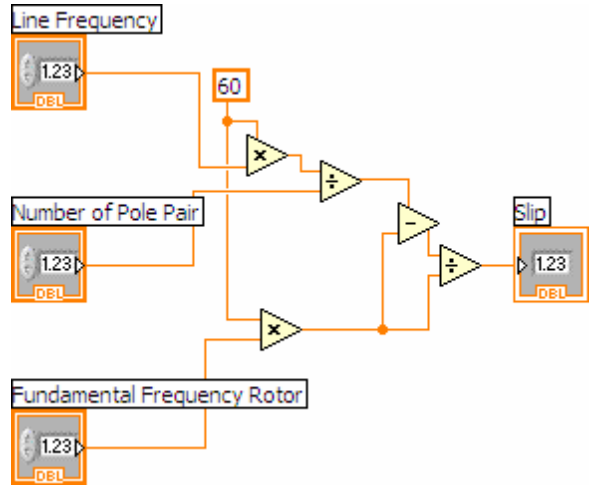

(b)

Figure 4.31 Sub VI of slip frequency calculation (a) icon and connector pane and (b) block diagram

Figure 4.31 illustrates that the VI has two input connectors to capture fundamental line frequency and fundamental rotor frequency. The VI then calculates the slip based on these frequencies using Equation 3.2 as illustrated in Figure 4.31(b). To demonstrate this technique, an example of the slip calculation is presented below for a three-phase induction motor $(415 \mathrm{~V}, 4.8 \mathrm{~A}, 2.2 \mathrm{~kW}, 4$ pole, with 32 rotor bars) when it is connected to a $50 \mathrm{~Hz}$ power line. The induction motor is coupled with dynamometer consisting of a $5 \mathrm{~kW}$ separately excited DC load. The result is presented in Figure 4.32 below.
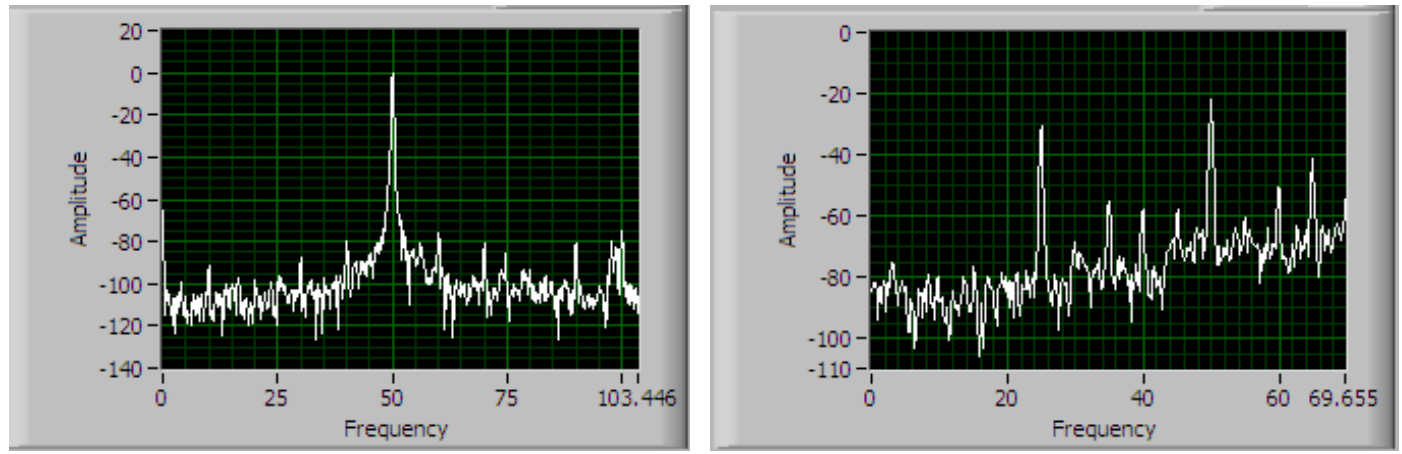

Figure 4.32 Waveform graphs of (a) fundamental line frequency captured by voltage monitoring, and (b) fundamental rotor frequency captured by vibration monitoring 
Figure 4.32 illustrates both fundamental frequencies with a light load. It can be observed in Figure 4.32(a) that fundamental line frequency was identified for voltage signals at $50 \mathrm{~Hz}$. In addition, the fundamental rotor frequency was found around 24.6 $\mathrm{Hz}$, as illustrated in Figure 4.32(b). The synchronous speed of the motor is

$$
\begin{aligned}
N_{S} & =\frac{120 \times F_{L}}{P}=\frac{120 \times 50}{4} \\
& =1500 \mathrm{rpm}
\end{aligned}
$$

For fundamental rotor frequency $24.6 \mathrm{~Hz}$ with light load, the rotor speed is

$$
\begin{aligned}
N_{R} & =F_{R} \times 60=24.6 \times 60 \\
& =1476 \mathrm{rpm}
\end{aligned}
$$

These speeds are then used to determine the slip of the induction motor.

$$
\begin{aligned}
s & =\frac{N_{S}-N_{R}}{N_{S}}=\frac{1500-1476}{1500} \\
& =0.016
\end{aligned}
$$

The slip of the induction motor is around $1.6 \%$. This slip can be used to determine various predictors of faults, especially for electrical-related faults such as eccentricity faults, broken rotor bar, and interturn stator-related faults. The calculation of the frequency predictors of each fault, including the LabVIEW VI, will be discussed in the following sections.

\subsubsection{Fault Frequency Predictors and The Sub VI}

\subsection{Eccentricity Fault}

In the case of eccentricity-related faults, three approaches can be implemented: Equation 3.20, Equation 3.22 and a frequency model shown in Figure 3.15. The first two approaches are implemented through stator current and flux leakage monitoring. The last approach is employed through vibration monitoring. Though all approaches are implemented in this research, Equation 3.22 may produce the best indication for mix eccentricity. The reason for this is that mix eccentricity usually occurs in real cases compared to single type eccentricity. This mix eccentricity can be identified using Equation 3.20. The implementation of Equation 3.22 using stator current or flux leakage may provide a better result than the frequency model in Figure 3.15 through vibration monitoring. The sub VI of the fault frequency predictor based on Equation 3.22 is shown in Figure 4.33. 


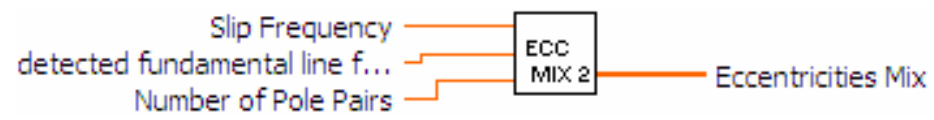

(a)

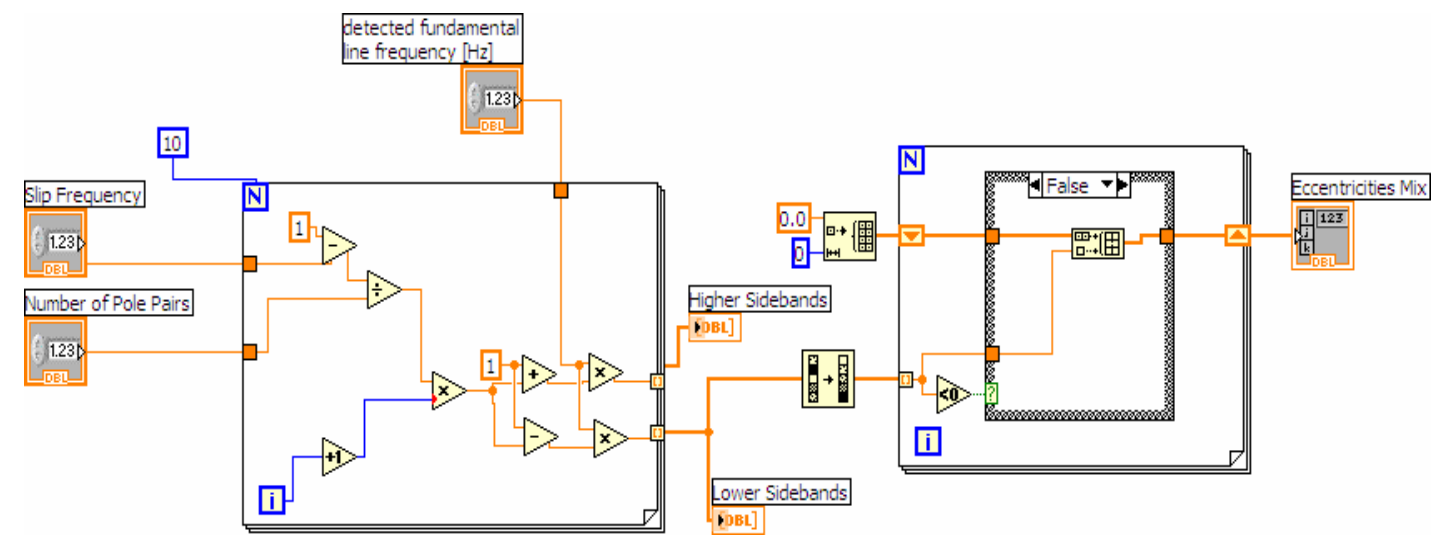

(b)

Figure 4.33 Sub VI of mix eccentricity calculation: (a) icon and connector pane and (b) block diagram

The sub VI of mix eccentricity shown in Figure 4.33 illustrates three input connectors and output of frequency predictors in 2D array numeric values. The input connectors are slip frequency, detected fundamental line frequency, and number of pole pairs. Sideband frequencies are calculated up to ten harmonics by eliminating negative frequencies. Using slip frequency obtained previously with pole pairs $=2$, the frequency predictors of eccentricity fault are given below.

$$
F_{E C C}=F_{L}\left[1 \pm k \frac{(1-s)}{p}\right]=50\left[1 \pm k \frac{(1-0.016)}{2}\right] \quad(k=1,2,3 \ldots 10)
$$

The sideband frequencies of eccentricity fault for

$$
\begin{aligned}
& k=1 \text { is } F_{E C C}=25.4 \mathrm{~Hz} \text { and } F_{E C C}=74.6 \mathrm{~Hz} \\
& k=2 \text { is } F_{E C C}=0.8 \mathrm{~Hz} \text { and } F_{E C C}=99.2 \mathrm{~Hz}
\end{aligned}
$$

Studies have highlighted that the lower sidebands (sidebands less than the fundamental line frequency) with $k=1$, in this case at $25.4 \mathrm{~Hz}$, can be a good indicator for the eccentricity faults $[51,58,80]$. Therefore, the threshold of severity level of mix eccentricity can be focused on this frequency.

\subsection{Broken Rotor Bar Fault}

For broken rotor bar fault analysis, Equations 3.15, 3.16 and 3.17 are utilized using several VIs as shown in Table 4.11. The Equation 3.16 can be used to represent the Equation 3.15 using $k=1$. The sub VI and block diagram of Equation 3.16 are presented in Figure 4.34. 


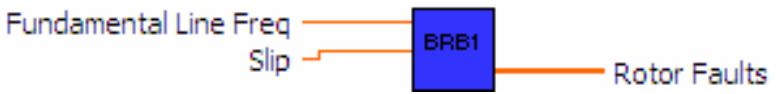

(a)

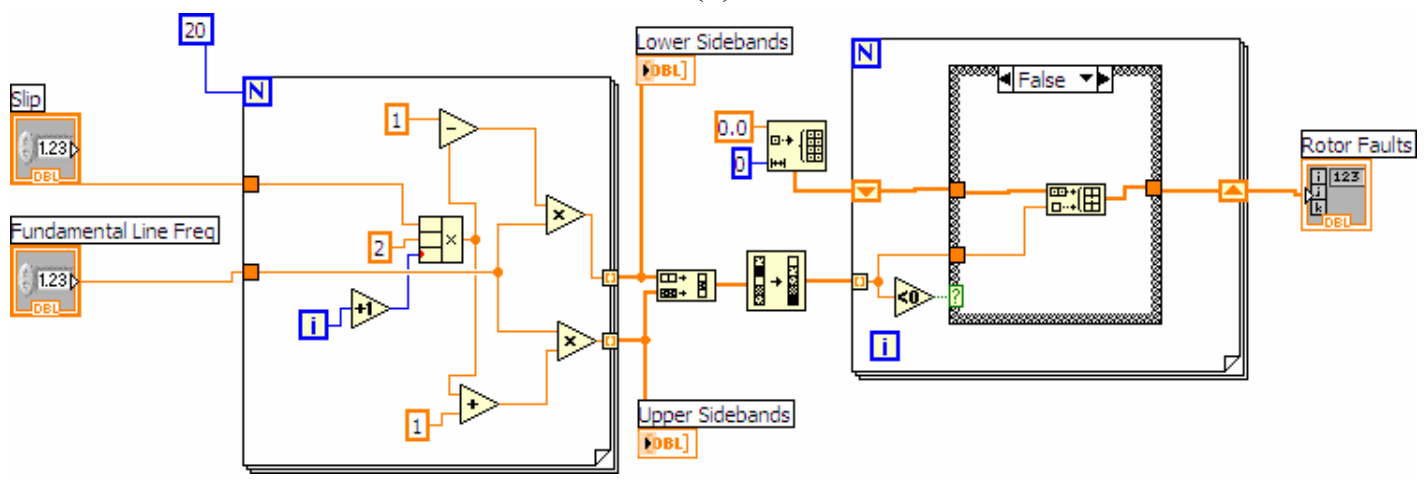

(b)

Figure 4.34 Sub VI of broken rotor bar calculation using Equation 3.16:

(a) icon and connector pane and (b) block diagram

Sub VI in Figure 4.34 shows three connectors: fundamental line frequency input, slip input, and 2D array values of rotor fault frequencies. Fundamental line frequency can be acquired from the voltage signal. The slip is obtained from previous calculations. The sub VI produces twenty lower sidebands of fault frequency more than $0 \mathrm{~Hz}$. In this sub VI both lower and upper sidebands are determined.

Studies $[49,125]$ indicated that these sidebands are affected by the load of the induction motor. The load affects the slip of the induction motor, which will in turn shift the sidebands. As an example, using slip frequency $s=0.016$ for light load and $\mathrm{s}$ $=0.046$ at full load, the lower and upper sidebands of the broken rotor bar fault frequency is presented below.

$$
\begin{aligned}
F_{B R B}=(1 \pm 2 k s) F_{L} & =(1 \pm 2 \times k \times 0.016) \times 50 \quad(k=1,2,3 \ldots 20) \\
& =(1 \pm 2 \times k \times 0.046) \times 50
\end{aligned}
$$

Table 4.12 Fault frequency predictor of broken rotor bar using Equation 3.16

\begin{tabular}{|r|r|r|r|r|}
\hline \multirow{2}{*}{$k$} & \multicolumn{2}{|c|}{$F_{B R B}(\mathrm{~Hz})$} & \multicolumn{2}{c|}{$F_{B R B}(\mathrm{~Hz})$} \\
\cline { 2 - 5 } & \multicolumn{2}{|c|}{ lower sidebands } & \multicolumn{2}{c|}{ upper sidebands } \\
\cline { 2 - 5 } & $s=0.016$ & $s=0.046$ & $s=0.016$ & $s=0.046$ \\
\hline 1 & 48.4 & 45.4 & 51.6 & 54.6 \\
\hline 2 & 46.8 & 40.8 & 53.2 & 59.2 \\
\hline 3 & 45.2 & 36.2 & 54.8 & 63.8 \\
\hline 4 & 43.6 & 31.6 & 56.4 & 68.4 \\
\hline 5 & 42 & 27 & 58 & 73 \\
\hline 6 & 40.4 & 22.4 & 59.6 & 77.6 \\
\hline
\end{tabular}


The result shows many sideband frequencies as predictors of broken rotor bars. The result also illustrates that the sideband frequencies change significantly when load or slip is varied. Regarding dominant sidebands, a study [49] highlighted that the lower sideband at $k=1$ usually has the highest amplitude of sidebands. Another study also suggested that both lower and upper sidebands at $k=1$ can be used as indicators [125]. It should be noted that these sideband frequencies do exist in healthy machines due to some degree of asymmetry because of intrinsic manufacturing variations. Research has pointed out that a broken rotor bar can be identified from certain amplitudes of these sidebands [49, 125]. Considering this experience, both the first lower and upper sidebands can be examined periodically using a threshold at specific amplitude based on separate base line studies.

Another approach for broken rotor bar detection is using Equation 3.17. The equation is implemented in a sub VI as shown in Figure 4.35. The sub VI has a data input connector pane similar to the previous broken rotor bar sub VI. The sub VI produces up to 15 sidebands. The sidebands are calculated only when $k / p=1,5,7,11$, 13 , etc... due to the normal winding configuration as suggested in [56]. As demonstrated in [56] lower sidebands can be utilized for a broken rotor bar predictor. Using light load and full load variation known previously, the fault frequency calculation and predictors are given below.

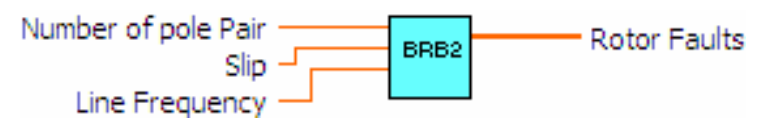

(a)

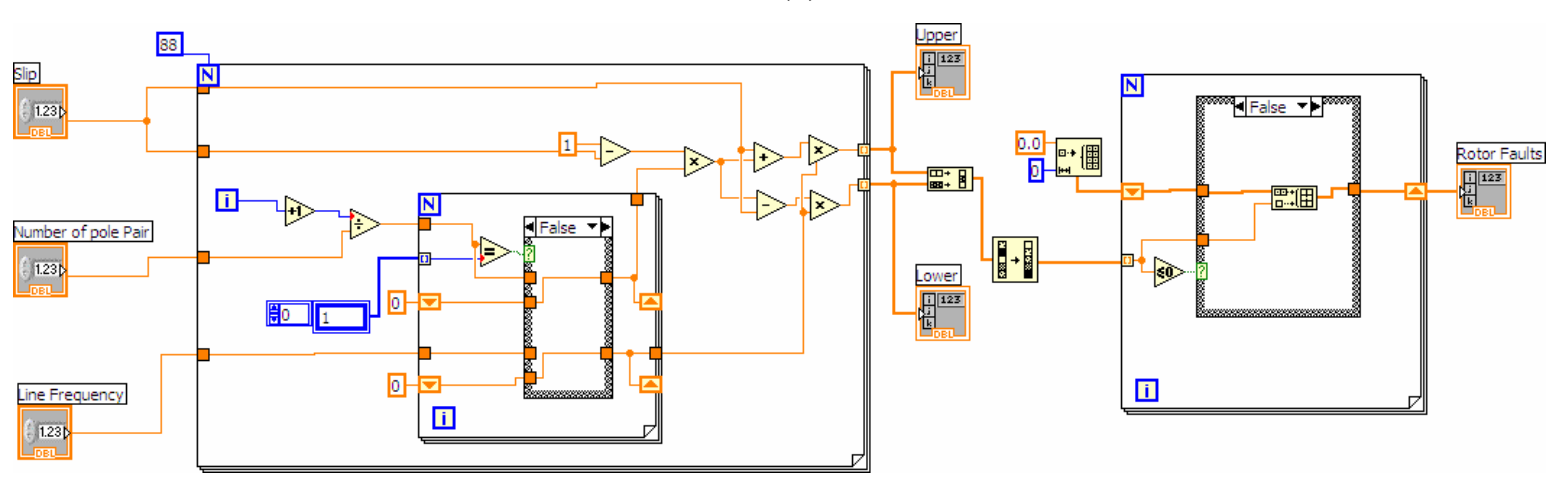

(b)

Figure 4.35 Sub VI of broken rotor bar calculation using Equation 3.17: (a) icon and connector pane and (b) block diagram 


$$
\begin{aligned}
F_{B R B}=\left[\left(\frac{k}{p}\right)(1-s) \pm s\right] F_{L} & =\left[\left(\frac{k}{p}\right)(1-0.016) \pm 0.016\right] 50 \quad(k / p=1,5,7,11,13 \ldots 43) \\
& =\left[\left(\frac{k}{p}\right)(1-0.046) \pm 0.046\right] 50
\end{aligned}
$$

Table 4.13 Fault frequency predictor of broken rotor bar using Equation 3.17

\begin{tabular}{|r|r|r|}
\hline \multirow{2}{*}{$k / p$} & \multicolumn{2}{|c|}{$F_{B R B}(\mathrm{~Hz})$} \\
\cline { 2 - 3 } & \multicolumn{2}{|c|}{ lower sidebands } \\
\cline { 2 - 3 } & $s=0.016$ & $s=0.046$ \\
\hline 1 & 48.4 & 45.4 \\
\hline 5 & 245.2 & 236.2 \\
\hline 7 & 343.6 & 331.6 \\
\hline 11 & 540.4 & 522.4 \\
\hline 13 & 638.8 & 617.8 \\
\hline
\end{tabular}

Both broken rotor bar predictors using Equation 3.16 and Equation 3.17 indicate similar frequency predictors in the first sideband. As well as the first sideband, the second sideband of each approach can be used as a fault predictor. The first and second sideband usually have a higher signal-to-noise ratio than other high harmonics. The higher harmonics have low spectrum amplitude and are sensitive to noise. Therefore, the first and second harmonics may provide reliable and discriminative information, especially for threshold analysis.

\subsection{Stator Shorted Turn Fault}

Another fault, stator shorted turn fault can be identified using Equation 3.14. The equation is implemented using a VI shown Figure 4.36. Similar to the previous VI, the VI captures the fundamental line frequency, and returns the array frequency of stator interturn faults.

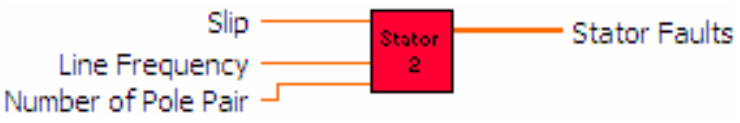

(a)

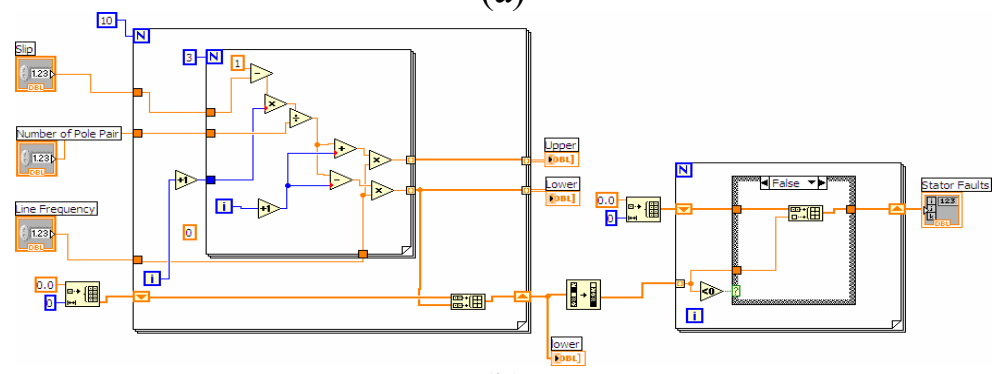

(b)

Figure 4.36 Sub VI of interturn fault calculation: (a) icon and connector pane, and (b) block diagram 
Figure 4.36 shows several key features of the sub VI. Firstly, a calculation is made up to 13 sideband predictors, for $n=1,2,3 \ldots 10$ and $k=1,2,3$. Secondly, the sub VI considers only the lower sidebands with positive values. Thirdly, the predictor of stator shorted turn fault frequencies is returned in a sorted one-dimensional array. The fault frequency predictor can be determined as given below using slip $=0.016$ and pole pairs $=2$.

$$
F_{S F}=F_{L}\left(\frac{n(1-s)}{p} \pm k\right)=50 \times\left(\frac{n(1-0.016)}{2} \pm k\right)
$$

Here $(k=1,2,3)$ and $(n=1,2,3 \ldots 10)$

\section{Table 4.14 Fault frequency predictor of stator interturn fault}

\begin{tabular}{|r|r|r|}
\hline & \multicolumn{2}{|c|}{$F_{S F}(\mathrm{~Hz})$} \\
\hline$n$ & $k=1$ & $k=2$ \\
\hline 1 & 74.6 & 124.6 \\
\hline 2 & 123.8 & 173.8 \\
\hline 3 & 173 & 223 \\
\hline 4 & 222.2 & 272.2 \\
\hline 5 & 271.4 & 321.4 \\
\hline
\end{tabular}

The fault frequency shown in Table 4.14 indicates that several frequencies can be considered for fault analysis. However, a study [49] suggested that the most detectable peak frequencies were $n=1$ or $k=1$. Other peaks might be useful to confirm the stator interturn related faults.

\subsection{Set-up Related Fault}

In the case of set-up related faults, a sub VI shown in Figure 4.38 is employed through vibration monitoring signals. The sub VI is developed based on various models of set-up related faults, as explained in Section 3.2.5. The set-up faults are mechanical unbalance, misalignment, and mechanical looseness.

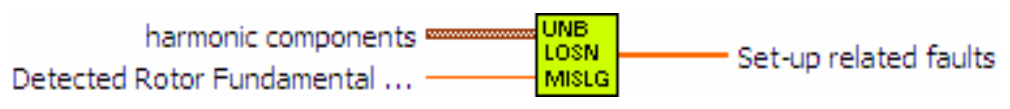

Figure 4.37 Icon of set-up related faults Sub VI 


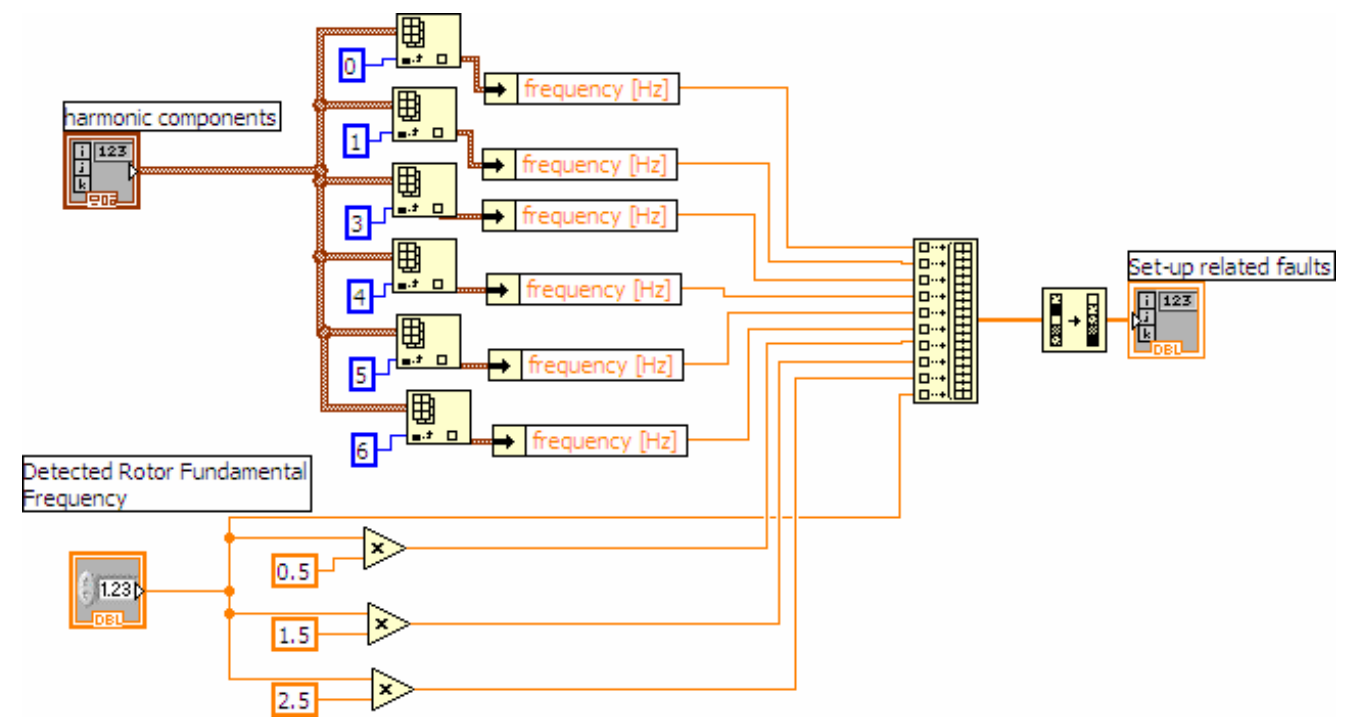

Figure 4.38 Block diagram of set-up related faults Sub VI

It can be seen from Figure 4.38 that the Sub VI is designed to represent various set-up faults. The sub VI captures detected fundamental rotor frequency $\left(F_{R}\right)$ through vibration monitoring. The Sub VI also acquires harmonic components of this fundamental frequency, including the amplitudes. However, in this sub VI the main concern is only the harmonic frequencies up to the seventh harmonic. The first three harmonics indicate predictors of mechanical unbalance and misalignment. Other harmonics determine mechanical looseness. The mechanical looseness can also be verified from the presence of $0.5 F_{R}, 1.5 F_{R}, 2.5 F_{R}$ which are generated by this sub VI as predictors.

\subsubsection{Limit Testing}

As can be seen in Table 4.11, besides all the fault frequency predictors, two limit testing VIs are important to determine the specific faults and severity levels. Two types of threshold are implemented in condition monitoring software. The first threshold is set up to determine all peaks more than a specific base line amplitude. The second threshold is applied to each specific frequency that is recognized through the previous threshold as severity level analysis. In this section, both thresholds will be discussed.

The first limit testing determines all frequency peaks higher than a specific threshold. In other words, the limit testing functions as peak searching of the FFT spectrum or spectra. In this step, peak searching is able to search peaks up to eight 
channel signals, and to return both frequency and amplitude. A VI that meets these requirements is presented in Figure 4.39.

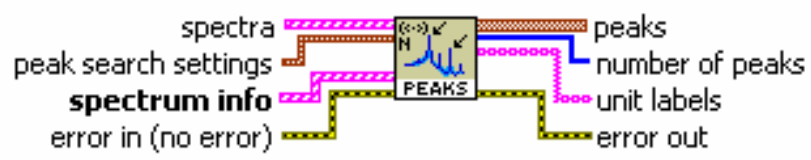

Figure 4.39 Sub VI of peak searching

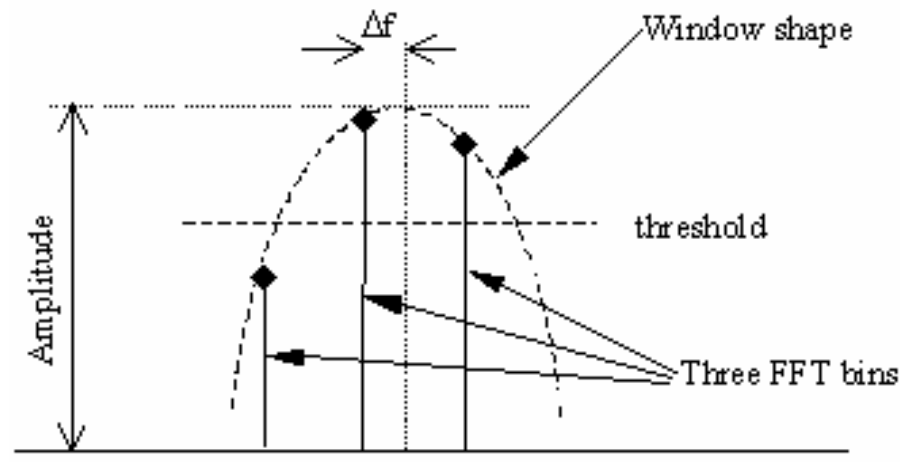

\section{Figure 4.40 Peak search technique of the sub VI in Figure 4.38[126]}

The peak searching VI shown in Figure 4.39 has several key features. This VI estimates the true tone of a peak frequency and amplitude using a curve-fitting algorithm. The curve-fitting maps a cosine window shape into at least three bins of a peak frequency, which indicates a local maximum of a power spectrum to determine the peak frequency, shown in Figure 4.40 [126]. The detectable peak frequencies above a threshold are returned as an array of peaks including the number of peaks and unit labels. This threshold can be adjusted according to the application. In this research the threshold of peak searching is fixed on $-60 \mathrm{db}$ as the general peak detection.

The second limit testing functions to examine all detected frequencies which are produced by the first threshold. This limit testing performs the diagnosis task as the severity level analysis. This limit testing step will determine whether or not frequencies are located between a lower limit and an upper limit. In other words, the limit testing will indicate when the amplitude of a peak frequency illustrates a good level, warning level, or a bad level in the case of a specific fault. Two main VIs are involved in executing the limit testing function: sub VI of limit creator and sub VI of limit testing, as shown in Figures 4.41and 4.42. 


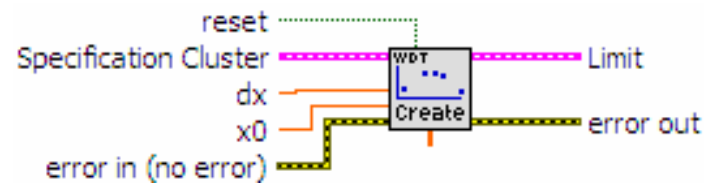

Figure 4.41 Sub VI of limit creator

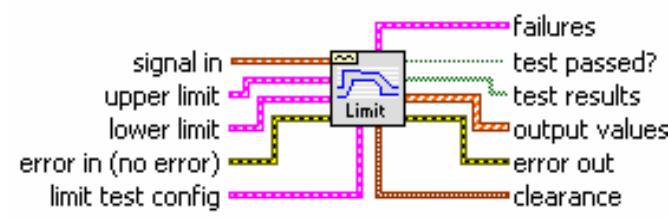

Figure 4.42 Sub VI of limit testing

The sub VI of limit creator shown in Figure 4.41 creates the limit as defined by input 2D array of $\mathrm{X}$ and $\mathrm{Y}$ values. The input array of $\mathrm{X}$ and $\mathrm{Y}$ values is acquired through the Specification Cluster connector shown in the figure. The input array $\mathrm{X}$ contains $\mathrm{X}$ axis values produced by the fault frequency predictors of a specific fault. The input array Y holds Y values that represent severity level. Each Y value can be determined manually by writing a certain threshold number according to the operator's experience or base-line studies. The VI will interpolate the data in the array $\mathrm{Y}$ at points $\mathrm{x} 0, \mathrm{x} 0+\mathrm{dx}, \mathrm{x} 0+2 \mathrm{dx}$ and so on, where $\mathrm{x} 0=\mathrm{X}[0]$. In the case of unconnected $\mathrm{x} 0$, the limit will be started from the first point in the $\mathrm{X}$ input array. To create lower limit and upper limit specification, two limit creator VIs are needed. Both limits are delivered to a limit testing sub VI.

The sub VI of limit testing shown in Figure 4.42 compares signal input, shown as signal-in connector, with upper limit or lower limit input. The limit testing sub VI will determine whether the input signals fall inside or outside both limits, known as limit mask testing. The limit mask testing returns true-value when the input signals are less than or equal to the upper limit, and more than or equal to the lower limit. The true or false value of the test-passed signal connector is generated including the test results in an array of Boolean values. Because of this, to indicate good, warning and bad levels, three sets of limit testing are needed for the development of these indicators. Detail of this development is presented in a sub VI shown in Figure 4.43. 


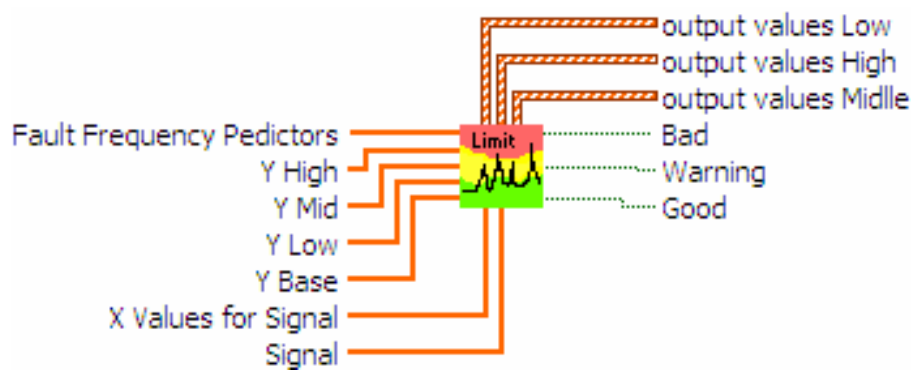

(a)

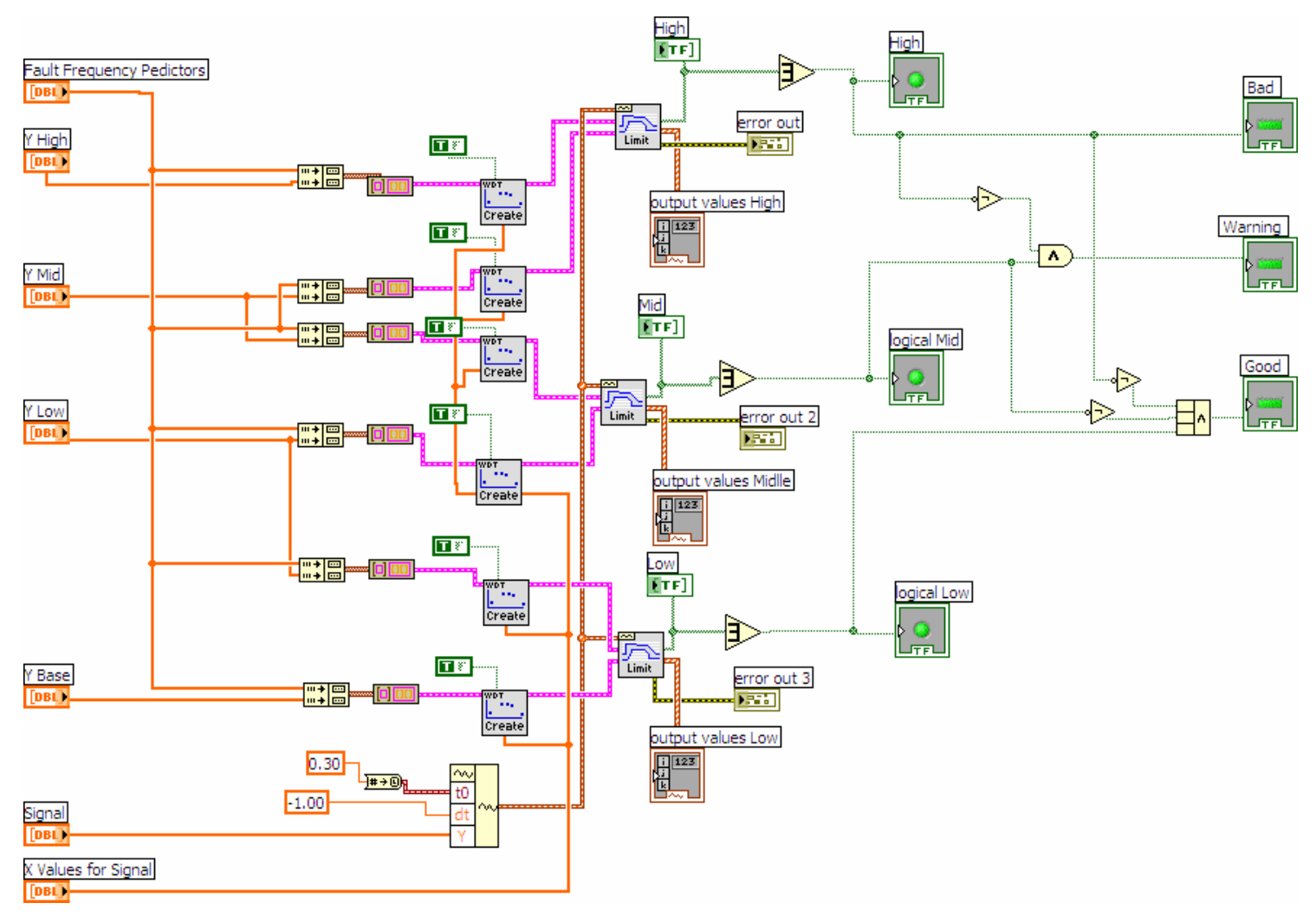

(b)

Figure 4.43 Sub VI of three limit thresholds of severity level (a) icon and connector pane and (b) block diagram

Illustrated in Figure 4.43, the sub VI of limit testing has four levels of limit. These four levels create three limit masks. The first limit mask is located between base threshold and the middle threshold. The limit mask between these two thresholds represents the good area. The low threshold is fixed at very low amplitude, at $-150 \mathrm{~dB}$. This value anticipates any lower amplitude or fake faults even though they are impossible to occur, as the peak searching threshold is fixed at $-60 \mathrm{db}$. The middle threshold can be adjusted for the warning level. The second limit mask is placed between the low threshold and the middle threshold. This limit mask specifies warning area. In the case of a peak being found between the middle and high threshold, the peak will be associated with a bad area. The high threshold is placed at 
$50 \mathrm{~dB}$. The result is presented as severity level using Boolean type. These Boolean logics will drive LEDs on a custom-made CompactRIO indicator module. The relation of these limit masks and severity levels is illustrated in Table 4.15, and an example of the result is given in Figure 4.44.

Table 4.15 Relation of the limit mask with the severity level area

\begin{tabular}{|c|c|c|c|}
\hline \multicolumn{3}{|c|}{ Limit mask (between) } & Level area \\
\hline base-low & low-middle & middle-high & \\
\hline TRUE & FALSE & FALSE & Good \\
\hline $\mathrm{X}$ & TRUE & FALSE & Warning \\
\hline $\mathrm{X}$ & $\mathrm{X}$ & TRUE & Bad \\
\hline
\end{tabular}

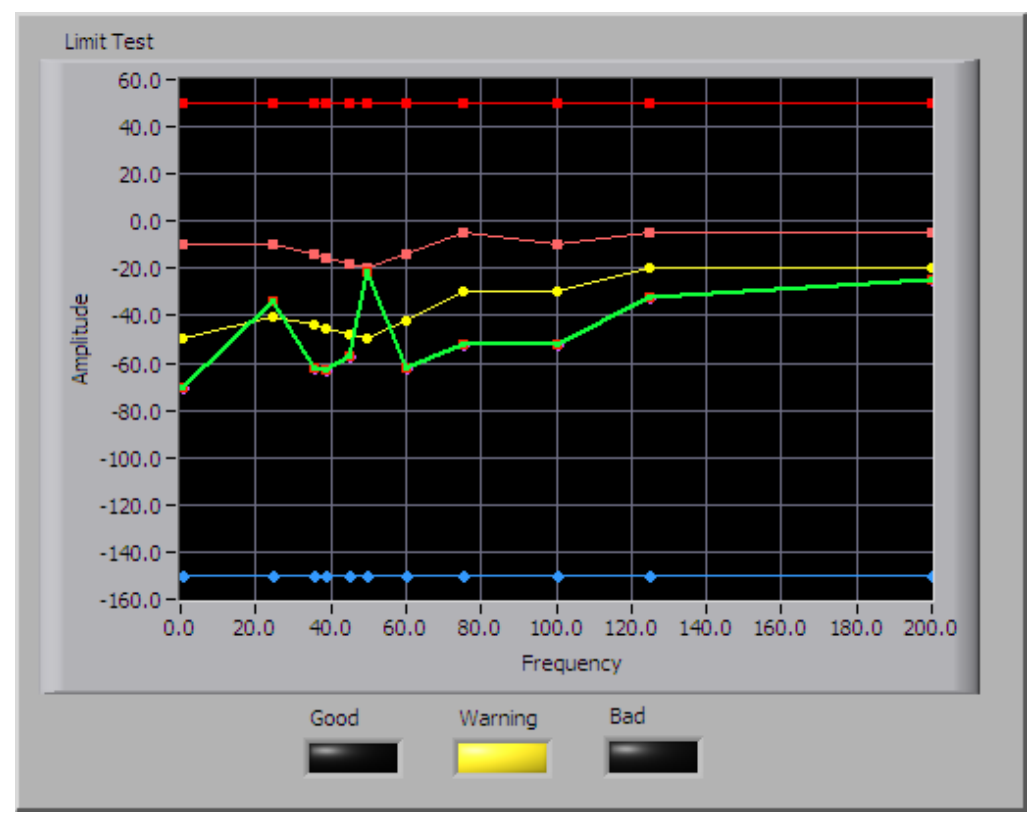

Figure 4.44 Example of limit testing result

Shown in Figure 4.44, the VI displays an example of the limit testing result for mechanical unbalance prediction. The limit testing VI classifies peak frequencies of a vibration signal shown in Figure 4.32(b). The frequency contains the fundamental rotor frequency and its harmonics. As illustrated in Figure 4.44, fundamental rotor frequency $24.6 \mathrm{~Hz}$ and the first harmonic can be examined by setting the low and middle threshold at certain amplitudes. Using this technique, the increased amplitudes of several fault frequencies can be monitored continuously.

The fault severity level of the induction motor produced by Limit Testing VI provides FPGA logic values to drive bicolours LEDs. To send these values, the severity level of each fault as shown in Figure 4.44 is converted using a simple VI, shown in Figure 4.45. 


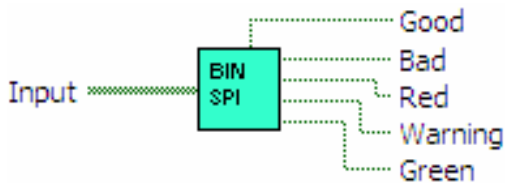

(a)

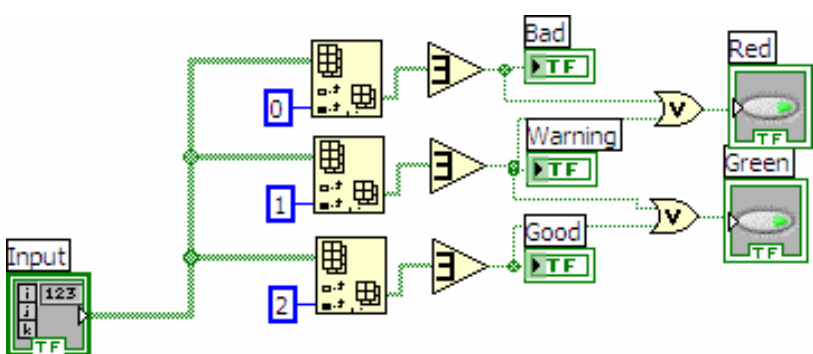

(b)

Figure 4.45 Sub VI of conversion from severity level to LED colours

Shown in this figure, three fault levels are converted into three colours. The Bad level is represented by red colour, good level by green and warning level by yellow (mixing up green and red colours). These colours are then converted into binary numbers which are transferred to FPGA. Details of the main program will be discussed in the following section.

\subsubsection{Main Program}

The main program of the condition monitoring software was designed following a flowchart in Figure 4.26. The main program is composed of all sub VIs discussed previously. The main program can be divided into two parts. The first part contains real-time host synchronization and frequency analysis. The host synchronization has been discussed in Section 4.5.1. The second part includes fault predictors, limit testing, and severity level writing. Both parts are presented in Figures 4.46 and 4.47 . 


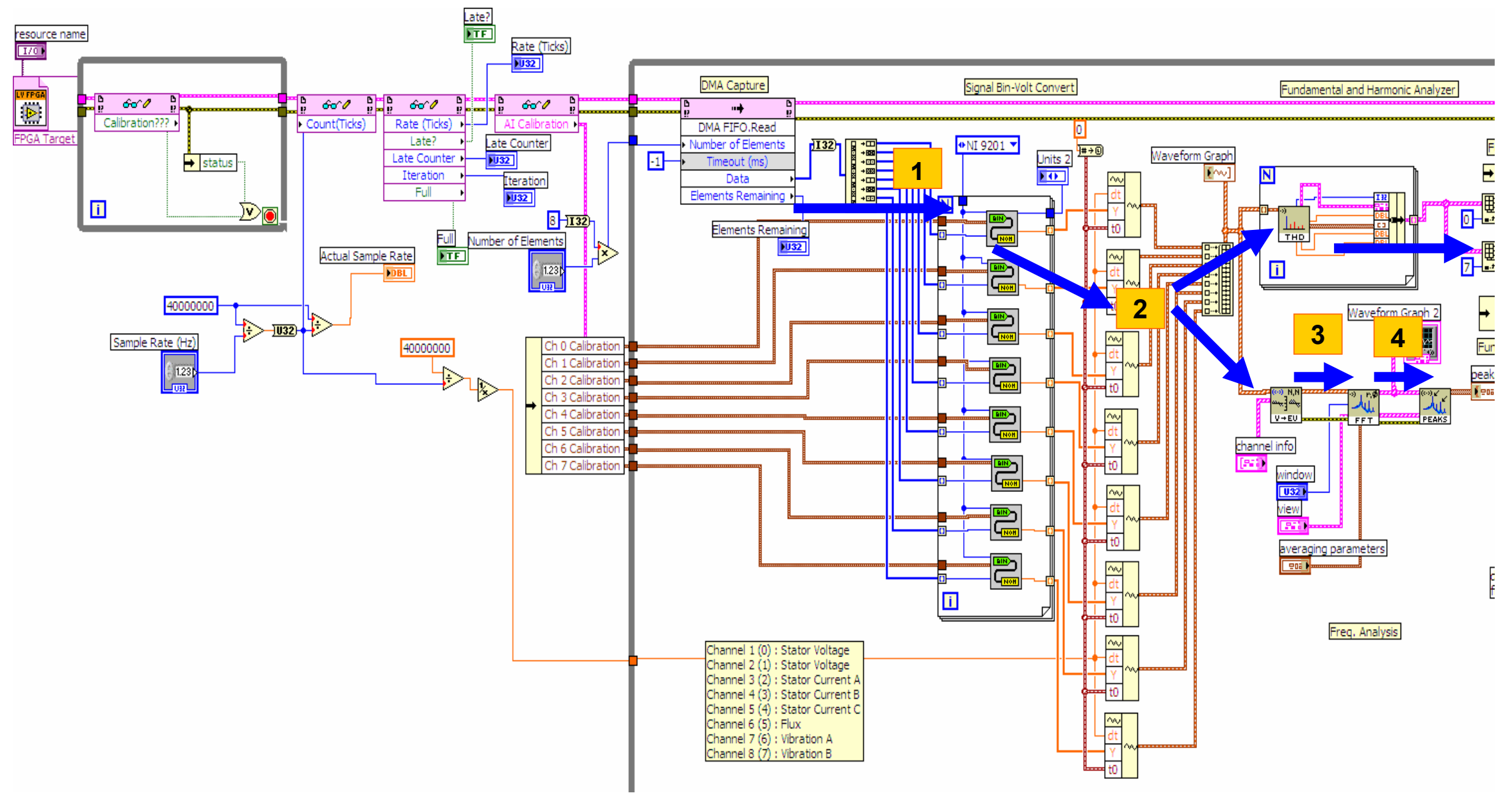

Figure 4.46 First part of main program of real-time condition monitoring 


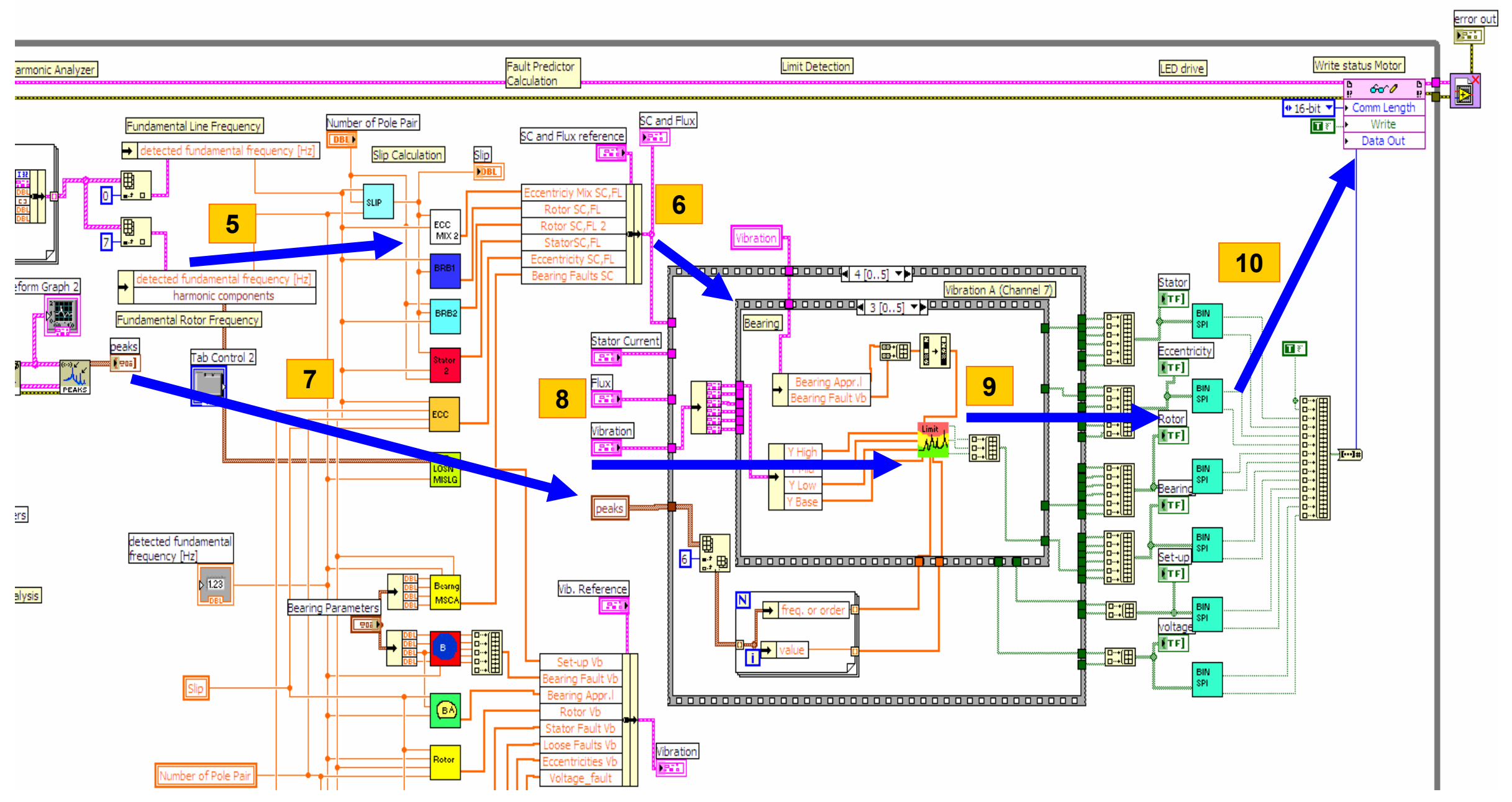

Figure 4.47 Second part of main program of real-time condition monitoring 
Figure 4.46 shows several functions of the main program: synchronizing the real-time software with FPGA, capturing signals through eight channels and performing frequency analysis. The first arrow illustrates the data flow of eight signals after captured-signals are received in the real-time host controller through FPGA. Sub VI of Binary to Voltage converts the binary captured-signals into voltage unit waveform using reference voltage range and calibration for NI 9201. The second arrow shows that the signals are delivered to sub VI of the engineering unit conversion and harmonic analysis. The sub VI of harmonic analysis allows the main software to determine fundamentals and harmonics of voltage signals and vibration signals. In the case of the second branch, the sub VI of Engineering Unit scales and normalizes the captured signal. The normalized-signals are fed to sub VI of FFT analysis as indicated by the third arrow. The sub VI of FFT analysis also includes smoothing windowing and frequency averaging. In this FFT sub VI, the eight channels frequency and amplitude spectrum is produced. The result of FFT signals is sent to a peak searching VI, as indicated by arrow four. In this VI, the peak search determines peaks higher than a specific threshold.

Shown in Figure 4.47, the main program carries out fundamental frequency selection, fault frequency predictor calculation, limit masking detection, and severity level to LED colours conversion. The VI of harmonic analysis produces fundamental and harmonic components. These components are utilized for the VI of fault frequency predictors. As indicated by arrow five, the VIs are slip, eccentricity, interturn stator fault, broken rotor bar, voltage and set-up related faults. In the next step, the fundamental frequencies, harmonic frequencies, and fault frequency predictors as illustrated by arrows six and seven, are employed for limit detection. The frequency predictors are categorized into stator current and flux leakage, and vibration. In addition to these frequency predictors, the limit maskings of severity level, shown in the beginning of arrow eight, are inputted manually to make an $(\mathrm{X}, \mathrm{Y})$ coordinate. The limit masking is carried out using a sequence case structure for six types of fault for each channel. Inside each case structure, the limit masking VI determines severity levels. The produced bad, warning or good indication is then converted into LED colours as binary number, and sent back to FPGA, as indicated by arrow ten. 


\subsection{Conclusions}

This chapter shows the development steps of the CompactRIO condition monitoring based unit. This chapter covers not only hardware development of the CompactRIO base package unit and the custom-made indicator module, but also the real-time condition monitoring software development. This chapter also discusses reconfigurable hardware utilization using LabVIEW FPGA, including SPI triggering technique for the custom indicator unit.

Based on comprehensive developments presented in each section, it can be summarized that the CompactRIO condition monitoring unit has covered all requirements for a complete condition monitoring unit. In other words, the CompactRIO has a reconfigurable FPGA hardware to capture signals through an analog input module, to drive an indicator module, and synchronize data communication with a real-time host controller. Furthermore, the CompactRIO has a host controller where condition monitoring software performs fault frequency analysis and determines threshold-based severity level. The CompactRIO also has a custom LED based indicator module, designed following the CompactRIO Module Development Kit (MDK) requirement. This indicator module aims to assist a lessexpert operators understand the severity level of each fault, based on the colour of each LED.

The development has been performed meticulously by considering several factors in each section for the best results. These factors are taken into consideration based on the literature and common practices in the hardware development of condition monitoring units of electrical machines. In order to recognize the CompactRIO condition monitoring performance, specific evaluations will be performed and discussed in the next chapter. 


\section{Chapter 5}

\section{Experimental Evaluation of the CompactRIO-Based Condition Monitoring System}

\subsection{Introduction}

To assure the correct performance and to verify the capabilities of the system developed, various tests were performed. These tests included the examination of execution times of each stage of the CompactRIO condition monitoring system program, and verification of the entire system (hardware and software) using real faults (single and multiple faults) introduced to the test motors.

This chapter is divided into three main sections. The first section describes performance evaluation based on execution times and real-time monitoring target resources. The second section demonstrates several measurements of standard sinusoid sources including several set-up parameters of frequency analysis mentioned in Section 4.5.2. In the final section, single and multiple faults are introduced to determine the capability of the condition monitoring system.

\subsection{Execution Time Evaluation}

As demonstrated in Chapter 4, the CompactRIO condition monitoring system was successfully developed through several steps. In order to determine how successful the developments and integration of the several modules are, the performance of this real-time system needs to be evaluated. Two basic techniques can be implemented to measure this performance: first is execution time analysis, and second is the real-time monitoring target. 
Execution time analysis was carried out using the Execution Trace Toolkit ${ }^{\mathrm{TM}}$ version 2.0 from National Instruments Inc [127]. In this test, the CompactRIO system captured a $200 \mathrm{~Hz}$ sinusoid signal from a signal generator. A development workstation containing LabVIEW Professional Development version 8.2.1 software and Execution Trace Toolkit version 2.0, was then connected to the CompactRIO system to capture the execution time whenever a session (a cycle between start and stop) of a measurement was finished.

The Execution Trace Toolkit is composed of two main tools: the execution trace tool and the execution trace tool VIs. Both should be used to obtain the results. The Execution Trace Tool VI is designed to capture the timing of VIs under test in a real-time target such as the CompactRIO system. This timing and event data are than displayed in the execution trace tool in a host computer or a development workstation.

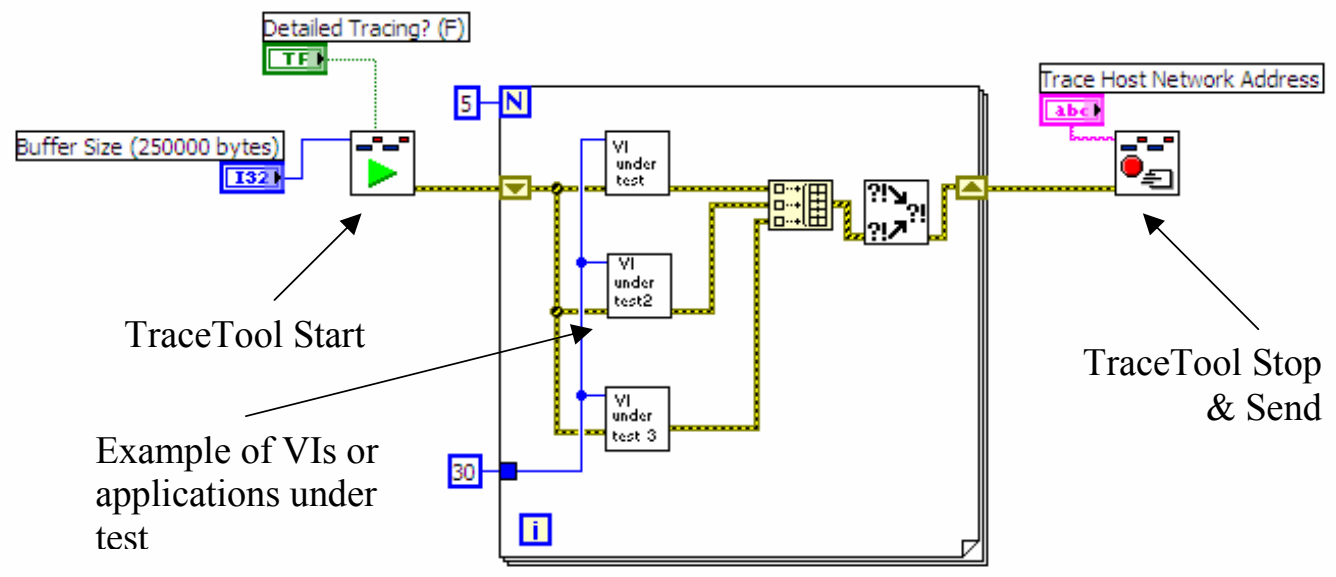

Figure 5.1 Execution Trace Tool VIs (see arrows)

Figure 5.1 shows two VIs (start and stop) to capture the timing and event, and an example of an application loop, in between these two VIs. After data is captured, the results are then sent to the execution trace panel to be displayed graphically, as shown in Figure 5.2. This figure shows the top window as a window of graphic-based analysis for the thread events, and the bottom graph for the VI events. The figure lists the event names and the sequence on the left side of each graphic based timing event. Each of the events can be zoomed for detailed analysis. 


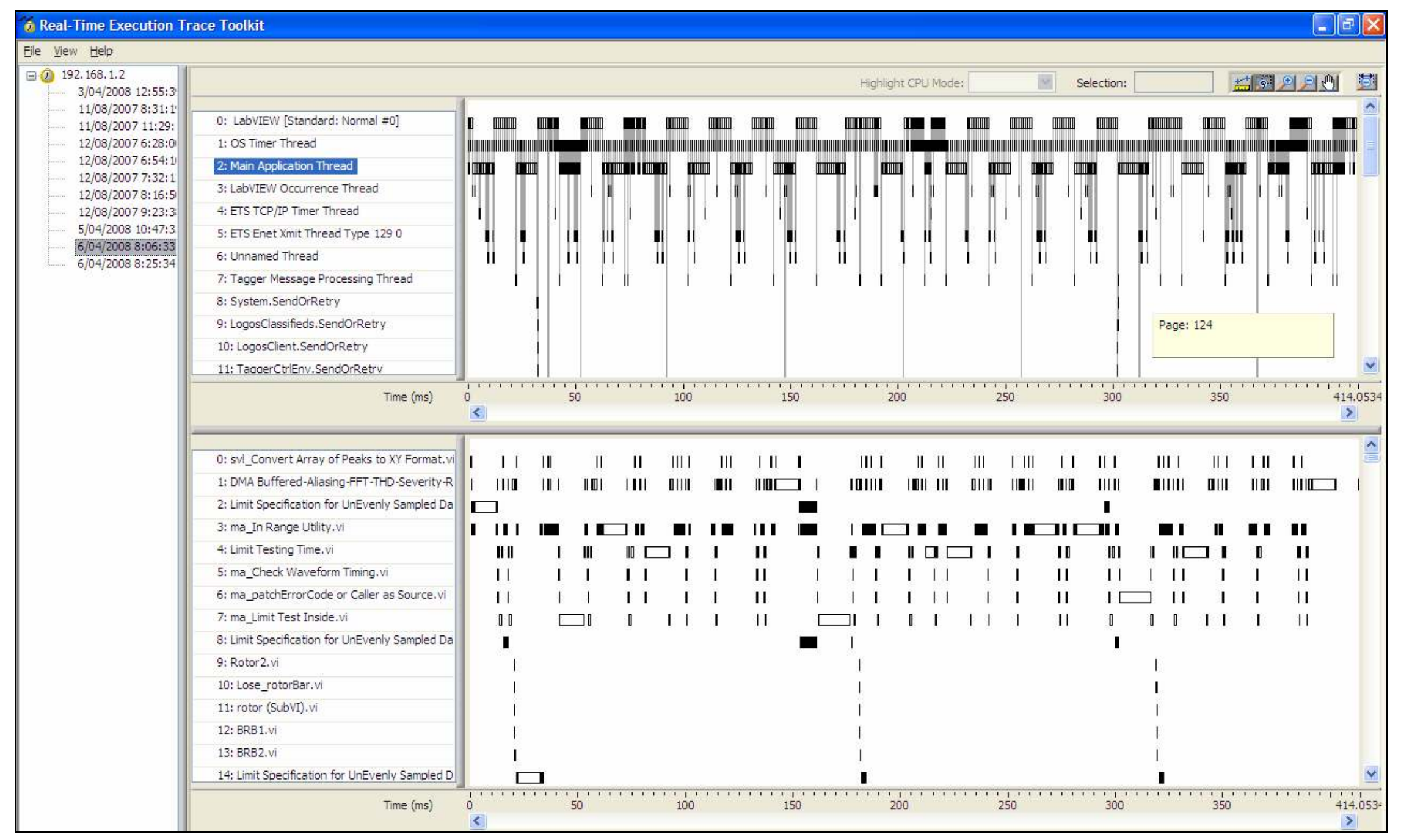

Figure 5.2 Execution Trace Tool front panel 


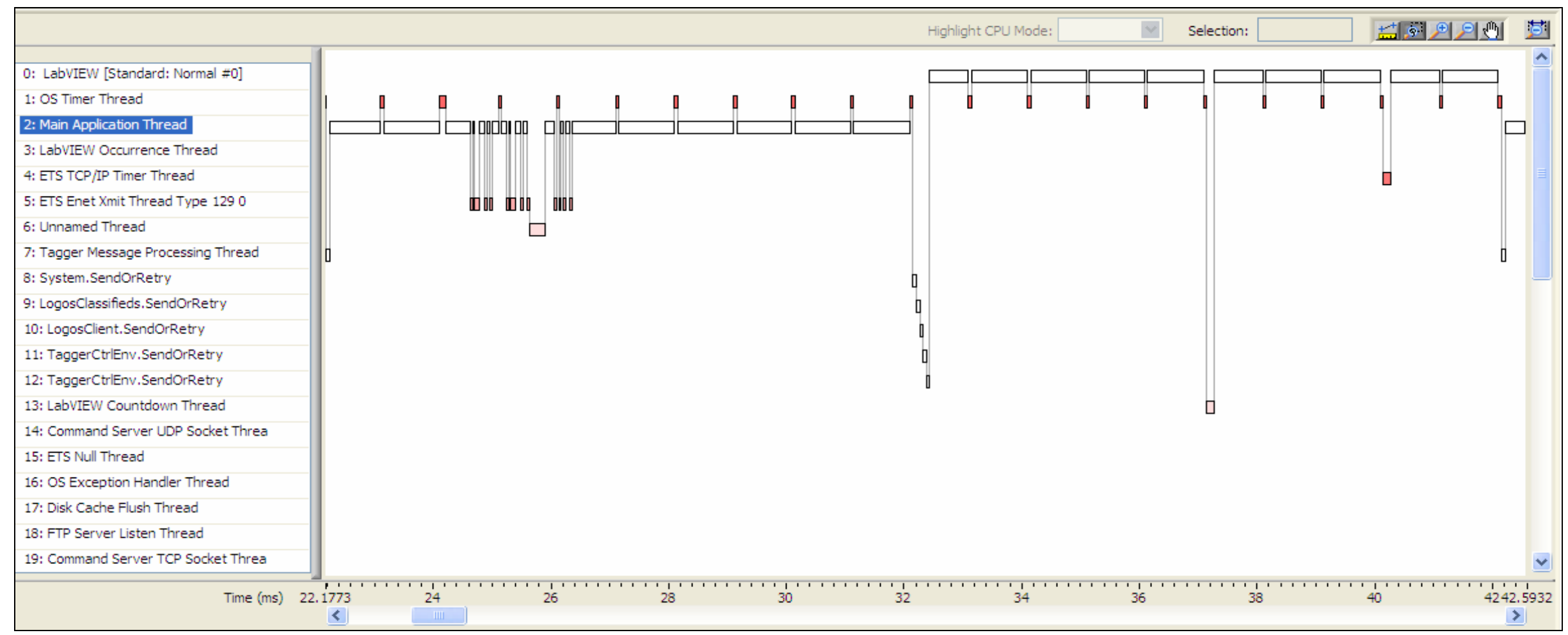

Figure 5.3 Zoom view of thread event of Execution Trace Tool demonstrates length of execution time and priority of each event according to colour 


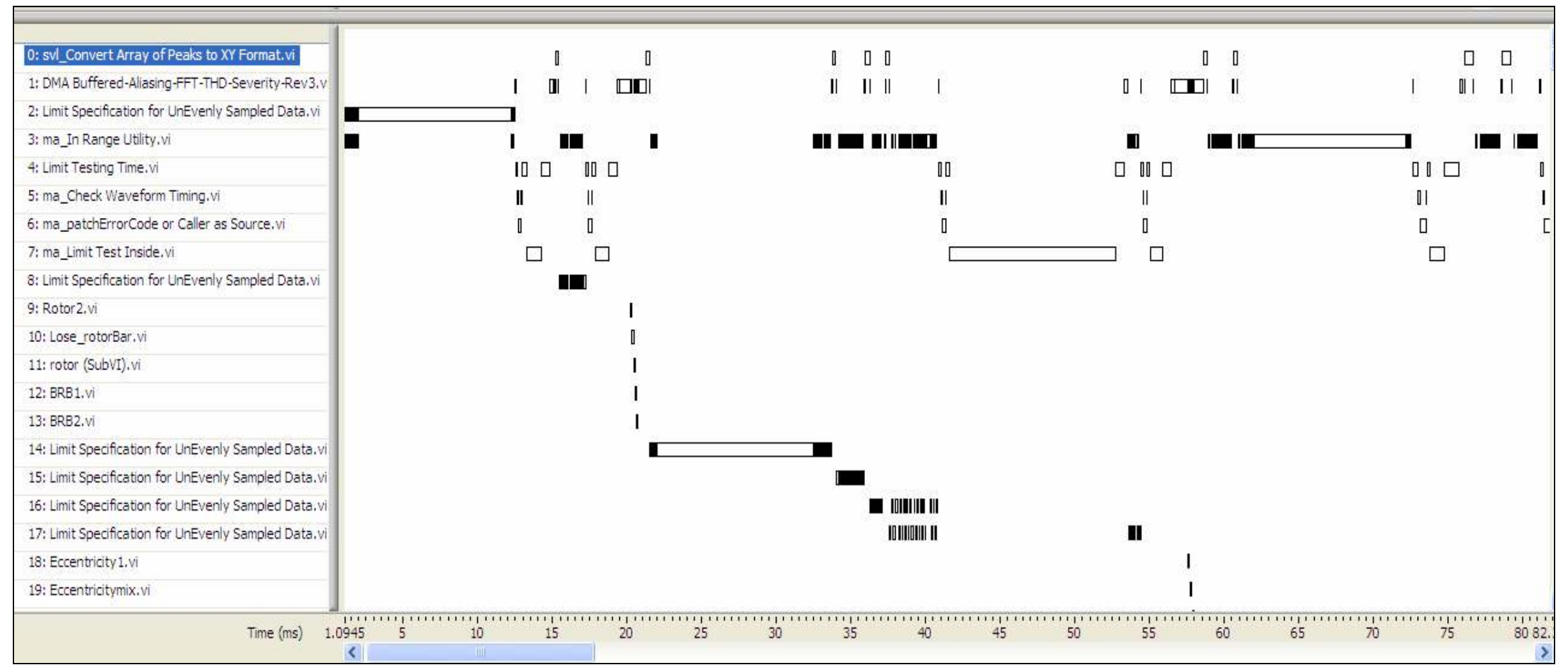

Figure 5.4 Zoom view of VIs event of Execution Trace Tool shows execution time of main and sub VIs 


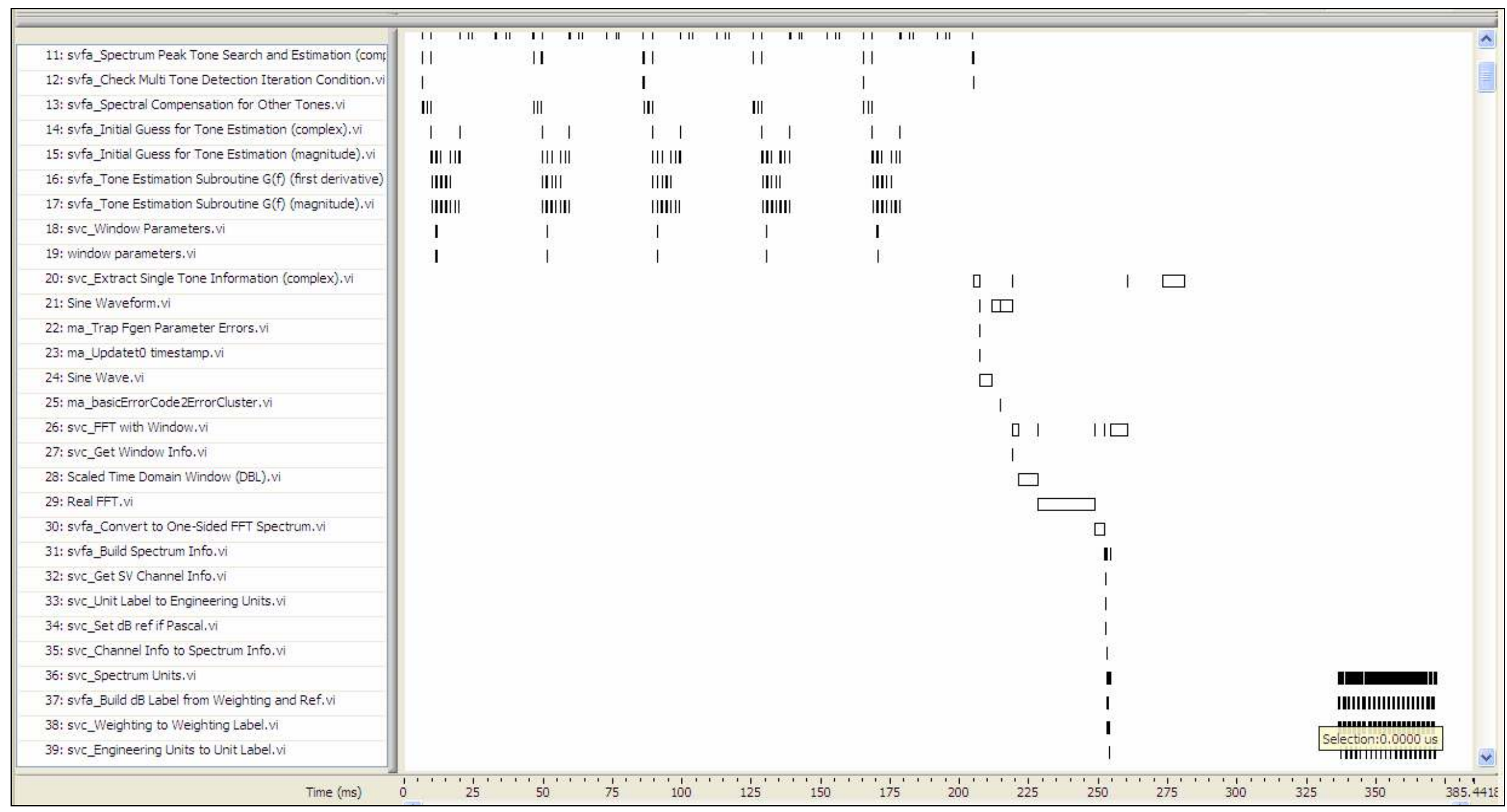

Figure 5.5 Zoom view of VIs event of Execution Trace Tool shows execution time of algorithms of frequency analysis 
Figure 5.2 shows the result of the execution time analysis of the performance of the CompactRIO condition monitoring system. In this test, the CompactRIO monitoring system captured standard sinusoid signals through eight channel analog input. The result was determined after the CompactRIO monitoring unit was run for several minutes to make sure a complete one cycle of measurement, including the frequency averaging function. This figure also shows that total execution time is 414 ms, which has been captured using $1 \mathrm{MB}$ buffer data from the Tracetool Start.vi of the Execution Trace Toolkit. The buffer of the Execution Trace Toolkit will capture data for $1 \mathrm{MB}$ data, regardless of how long the real-time software performs a cycle of signal capturing for the eight channels. In other words, the total execution time represents the total time spent to acquire $1 \mathrm{MB}$ data. During the execution time, realtime application software can complete no cycles, single or multiple operation cycles. The advantage is that the total execution time helps in understanding clearly all VIs or threads and in determining the cycle of each data capturing. In order to illustrate the execution time of the VIs and threads, the zoom view of the thread event window is presented in Figure 5.3.

Table 5.1 Description of several thread events [128]

\begin{tabular}{|l|l|}
\hline \multicolumn{1}{|c|}{ Thread Name } & \multicolumn{1}{c|}{ Description } \\
\hline Main Application Thread & A thread that handles front panel communication \\
\hline OS Timer Thread & A thread that handles timer for operating system \\
\hline $\begin{array}{l}\text { LabVIEW Thread [execution } \\
\text { system }]\end{array}$ & $\begin{array}{l}\text { A thread that runs one or more VIs in a LabVIEW } \\
\text { execution system. Execution system is the } \\
\text { LabVIEW execution system where the thread } \\
\text { executes }\end{array}$ \\
\hline ETS Enet Xmit Thread & A thread that transmits data to the Ethernet driver \\
\hline Unnamed Thread & A thread that handles operation system task \\
\hline ETS Null Thread & An idle execution thread \\
\hline ETS TCP/IP Timer Thread & A thread that handles TCP communication \\
\hline
\end{tabular}

As pointed out in Figure 5.3, the execution of thread events in a real-time CompactRIO target comprises the main events as described in Table 5.1 above. Each of the threads represents both operating system routines in a real-time system. The real-time system target only executes one thread at a certain time. For example, the TCP/IP timer thread which handles TCP/IP communication between the real-time host controller and the development workstation is around $102 \mu \mathrm{S}$, and executed almost every $35 \mathrm{~ms}$. 
Figure 5.3 also shows the priority execution of each function or VI in the realtime condition monitoring software. As shown in the Figure 5.3, the operating system timer thread is the highest priority or time critical, as indicated by the red colour, followed by the ETS Enet Xmit Thread as the next priority (dark pink), and abovenormal priority as indicated by the unnamed thread (light pink). The main application thread and VIs of LabVIEW-based condition monitoring are at normal priority. These results show that the timer and operating system of the real-time host must have the highest authority to ensure operations. An interesting point in this case is that the Ethernet thread also has a high priority because it is employed extensively during the test to transfer data to be displayed in the development workstation. It is believed in the case of the stand-alone applications (without utilizing Ethernet connection for data distribution) such as implemented in this research that the priority of the Ethernet thread will be lower. However, unfortunately this cannot be proved because the Ethernet thread will be used every time the Execution Trace Toolkit needs to be used.

It can be interpreted from Figure 5.3 that the execution time of all routine tasks for the real-time operating system is relatively short. This time is around $470 \mu \mathrm{S}$, compared with the LabVIEW thread, i.e. around $10 \mathrm{~ms}$ in each cycle block, or in the Main Application Thread, also around $10 \mathrm{~ms}$. In order to scrutinize which is the biggest contributor to this long execution time, each application VI that contributes to the LabVIEW thread and Main Application thread was examined.

Further investigation of each VI performance can be done by zooming Figure 5.2. The result is presented in Figure 5.4. This figure indicates that the longest execution time in the Main Application thread is the VI of Limit Specification for Unevenly Sampled. This is then followed by the VI of limit testing. Other sub VI events which are part of the limit testing, such as the sub VI for converting the array of peaks to $\mathrm{X}$ and $\mathrm{Y}$ axis and the sub VI for in-range matrix checking, are also contributors for the length of execution time in the LabVIEW thread. It should be noted from this test that the most active task is the sub VI of matrix in the range for limit testing, followed by the main application VI. This indicates that the excessive execution of this sub VI which is supposed to be lower than the main VI, might have been running improperly, and delayed the main VI of the real-time condition monitoring software. 
Figures 5.2 and 5.4 also indicate the other VIs implemented in fault analysis. Each fault analysis VIs such as eccentricity, broken rotor bars, bearings, and others, is performed in a relatively shorter time than the other VIs. This relatively short execution time also occurs in the VI of the real-time main application. It can be concluded that there are significant differences in execution time between the VI and the sub VIs of the limit testing category and other algorithms implemented. This difference might indicate an immature code of these VIs of limit testing used in the real-time environment.

As can be seen in Figure 5.2, the execution time of the signal processing algorithms, as listed in Table 4.8, is not displayed clearly. Therefore, in order to understand the performance of these frequency analysis algorithms, another focus of execution time analysis is carried out, illustrated in Figure 5.5.

The execution time for simultaneous 8-channel data acquisition, including cutoff filtering, was found to be less than $1 \mathrm{~ms}$. The maximum execution time of the various sections of the signal processing algorithms for each channel is listed in Table 5.2. Table 5.3 indicates the execution time of the fault detection and diagnosis algorithms.

Table 5.2 Average execution times of the various sections of the signal processing algorithms

\begin{tabular}{|l|l|}
\hline Algorithms & Execution Time \\
\hline $\begin{array}{l}\text { Engineering unit } \\
\text { adjustment }\end{array}$ & $0.3 \mathrm{~ms}$ \\
\hline Windowing & $8 \mathrm{~ms}$ \\
\hline FFT analysis & $6 \mathrm{~ms}$ \\
\hline Averaging & $5 \mathrm{~ms}$ \\
\hline Peak searching & $3 \mathrm{~ms}$ \\
\hline $\begin{array}{l}\text { Harmonic and fundamental } \\
\text { analysis }\end{array}$ & $2 \mathrm{~ms}$ \\
\hline
\end{tabular}

Table 5.3 Average execution times of the CompactRIO fault analysis stage

\begin{tabular}{|l|l|}
\hline Algorithms & Execution Time \\
\hline $\begin{array}{l}\text { Bearing, set-up, stator, rotor, } \\
\text { eccentricities, supply faults } \\
\text { frequencies model calculations }\end{array}$ & $2 \mathrm{~ms}$ \\
\hline $\begin{array}{l}\text { Limit testing of bearing, set-up, } \\
\text { stator, rotor, eccentricities, and } \\
\text { supply faults }\end{array}$ & $25 \mathrm{~ms}$ \\
\hline
\end{tabular}


Tables 5.2 and 5.3 illustrate the length of the execution time of each function in real-time condition monitoring software. As can be seen in Table 5.2, the fault detection and the diagnosis algorithm for limit testing have the longest execution time compared with the other algorithms. Other algorithms are executed for less than 10 $\mathrm{mS}$. For an 8-channel monitoring, the total execution time, including signal processing, fault analysis, and CompactRIO standard operating system tread (such as events for FTP data transfer, opening and closing applications) took approximately $164 \mathrm{~ms}$.

Although the execution times of the algorithms mentioned above are acceptable for the condition monitoring application, further improvements can be made, for example by improving the limit detection algorithm that is based on an interpolation method. Furthermore, the memory and CPU resources of the real-time target can be evaluated to show the performance of the CompactRIO system dealing with condition monitoring software, as shown in Figure 5.6.

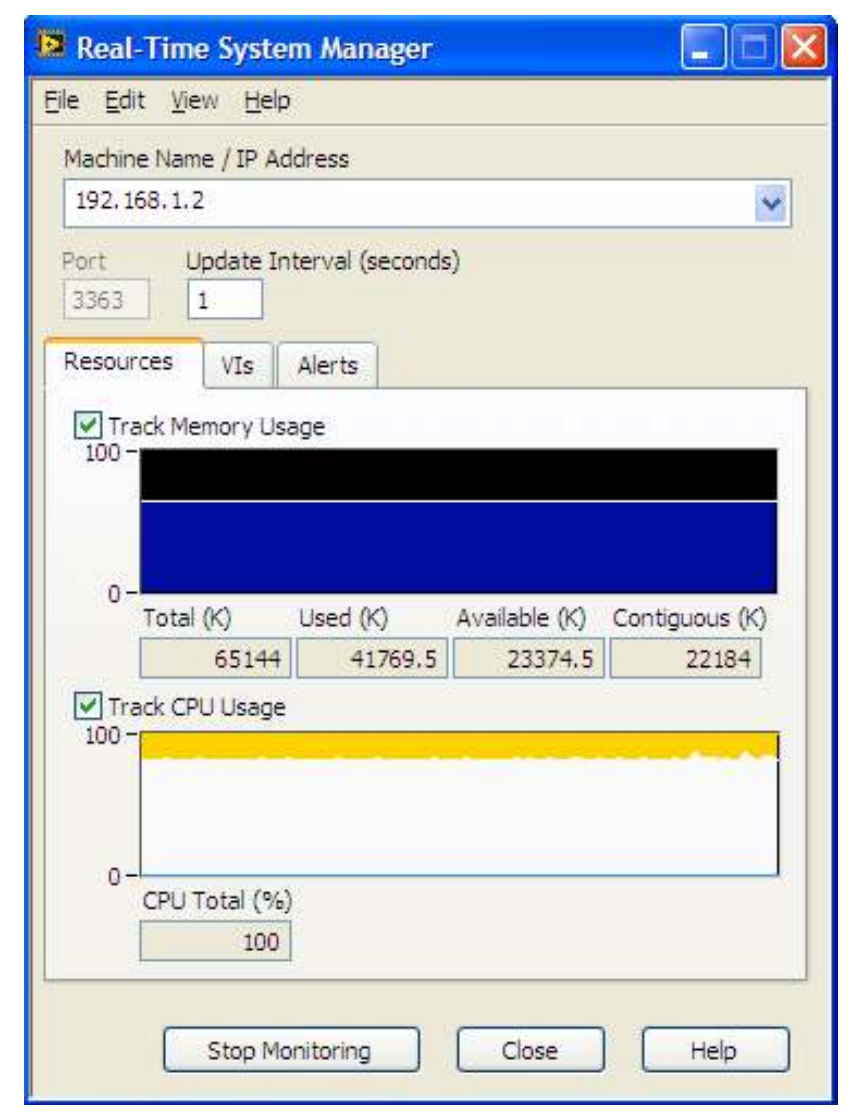

Figure 5.6 Real-time system manager shows memory usage and CPU usage during a measurement 
As stated previously memory and real-time CPU usage can be monitored during the operation of the CompactRIO condition monitoring system. Figure 5.6 can be used to point out that the condition monitoring application have consumed almost all the memory available. The figure shows that the system utilizes around $64 \%$ of the total memory with low priority tasks, as indicated by the blue colour in the memory usage graph. This figure also shows that the CPU is forced to utilize $100 \%$ of its resources. This high CPU consumption is illustrated with an above normal activity graph, as designated with the yellow colour graph. This demonstrates that all resources in the real-time host controller have been employed. To minimize this consumption of CPU and memory resources, it would be better if some or all of the specific tasks of the real-time condition monitoring software, which are developed in LabVIEW Real Time software, can be moved to FPGA. For example, FFT processing including windowing, averaging, and peak searching algorithms might be written in LabVIEW FPGA. A new version of LabVIEW 8.5 has covered the FFT VI in FPGA development. These strategies can offer faster overall performance than is currently presented, while the real-time host controller can be focused on enriching the fault detection performance.

\subsection{Frequency response evaluation}

As mentioned in the introduction to this chapter the next performance analysis was carried out by introducing standard sinusoid signals to the CompactRIO condition monitoring system. This analysis is undertaken in order to understand the behaviour of the system performing measurement and analysis for a known signal.

Similar to the first test, a sinusoid signal $140 \mathrm{~Hz}$ was introduced to the system through the analog input module. The CompactRIO system was taken connected to a development workstation using the Ethernet connection. After condition monitoring was uploaded, as described in Section 4.4.3, the results of the frequency analysis that can be seen from the front panel of the LabVIEW program were captured by the development workstation.

The first test was done by comparing input signals of the sinusoid signals before and after low-pass filtering was performed. This examination can be used to determine the behaviour of signals after the critical filtering stage. As detailed in Section 4.4.1, the low pass filter has been successfully developed through several 
steps. In order to show the result of the design, a test was performed, as shown in Figure 5.7.

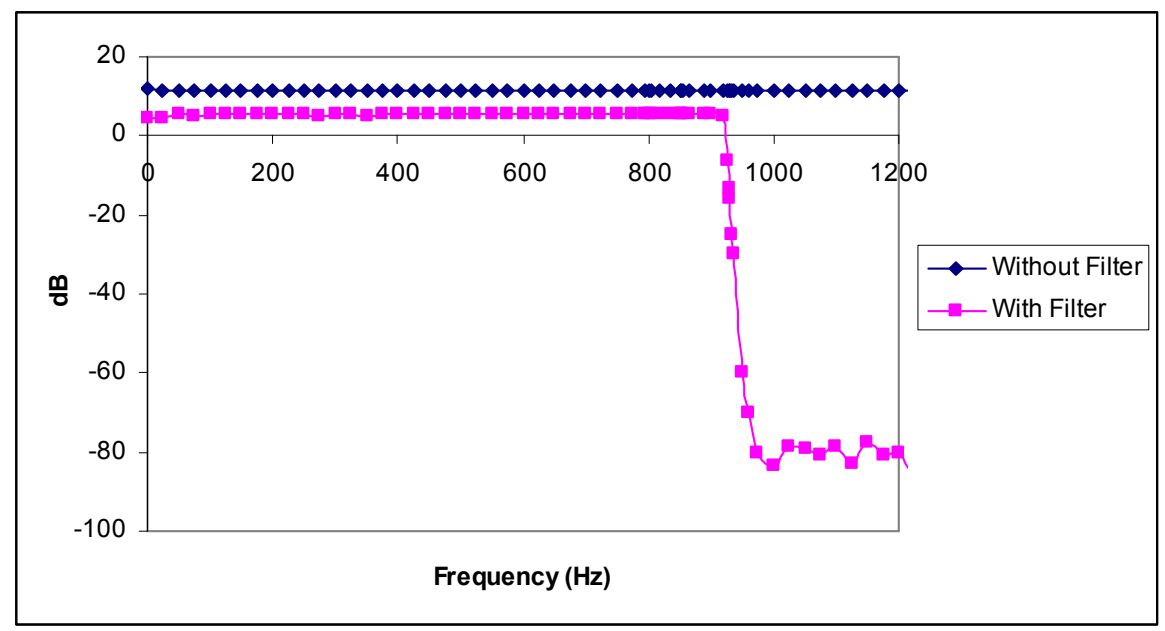

Figure 5.7 Frequency response of monitoring system without low pass filter and using low pass filter

The frequency response in Figure 5.7 shows that all frequencies higher than the edge of stop band are cut-off. The top line graph illustrates the frequency response of sinusoid signal without filtering and the bottom line graph shows the frequency response after low pass filter. Shown in the figure, the pass-band frequency is relatively flat. In addition, the edge of stop band frequency is around $920 \mathrm{~Hz}$ because of sub sampling. The transition band is relatively sharp, around $25 \mathrm{~Hz}$. As can be seen from the figure, the stop band amplitude is around $-80 \mathrm{~dB}$.

The graph also shows that attenuation occurs, as indicated from the reducing of amplitude before and after low pass filtering. Such attenuation may occur after filtering. In order to minimize this attenuation effect several configurations of Butterworth low pass filtering have been tried. As far as experiences during the trial of several configurations, the author cannot obtain the filtered signals which have similar amplitude with the source signal. This is despite the fact that the Digital Filter Toolkit simulation has proved the closeness of behaviour between the floating and fixed-point filter. The attenuation might be because the fixed-point code which has been utilized in the digital filter design, as indicated in Section 4.4.1, needs further development to be implemented in FPGA.

Despite the attenuation, the filtered signal itself is still acceptable for fault analysis. This is because the magnitude of the amplitude can still be used to represent the severity level of faults. In other words, it can be demonstrated that the filter is 
capable of producing signals with similar frequency and form, compared with the source. This is an essential requirement as is seen later when detecting specific frequency compared with fault frequency.

The following graphs show various windowing and resolution settings that have been implemented. Figures 5.11 to 5.14 show that the CompactRIO condition monitoring software can be set using various FFT resolutions. This test was done using a $140 \mathrm{~Hz}$ sinusoid signal that was sampled at frequency $2000 \mathrm{~Hz}$ and normalized to the highest peak frequency.

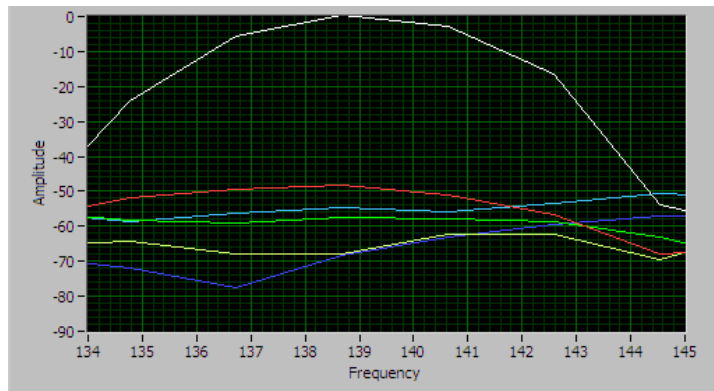

(a)

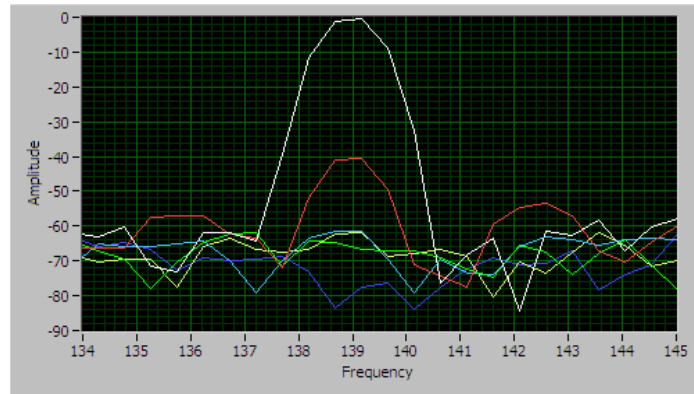

(c)

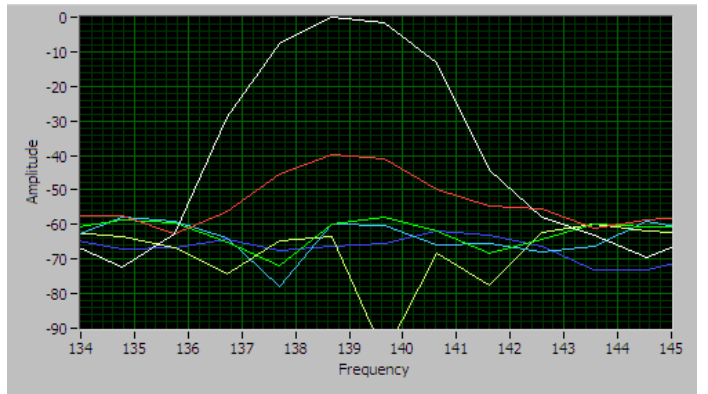

(b)

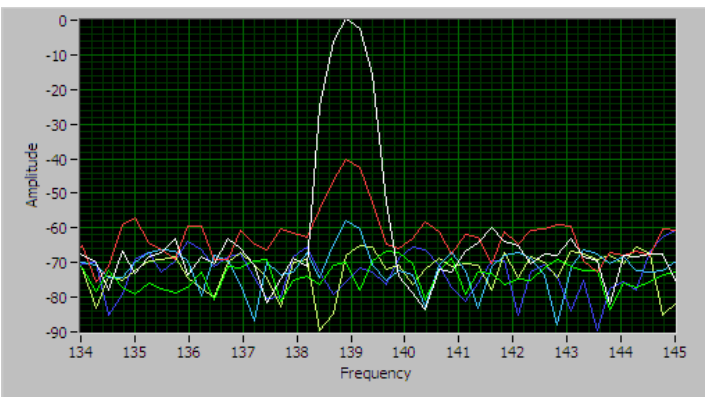

(d)

Figure 5.8 Zoom view of specific peak with : (a) 512 lines (b) 1024 lines (c) 2048 lines and (d) 4096 lines

FFT resolution can determine how successfully two close frequencies can be separated. As pointed out in these figures, the CompactRIO system is capable of performing a maximum 4096 FFT lines. This can be understood because the the CompactRIO analog input NI 9210 has 12 bit ADC to convert the 8 channels analog signals into the digital form through a multiplexer. However, this 4096 FFT lines is still acceptable to differentiate two close frequencies such as the sideband frequencies around the fundamental line frequency. Further detailed analysis, such as high resolution frequency, is needed when detailed analysis toward a specific fault frequency is to be implemented. In this case, NI 9205, NI 9215 or NI 9229, which 
have a high bit ADC and simultaneous sampling, can replace the NI 9210 with little modification of FPGA software.

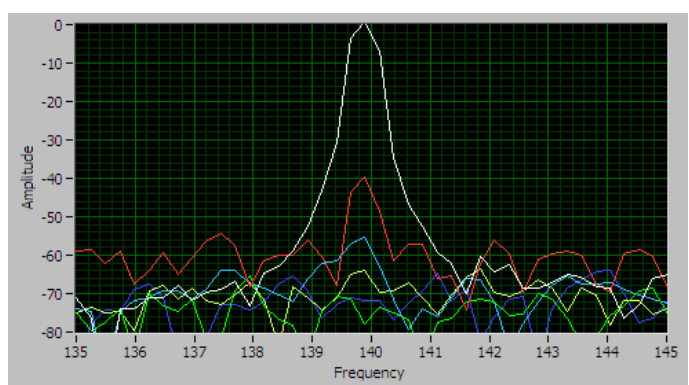

(a)

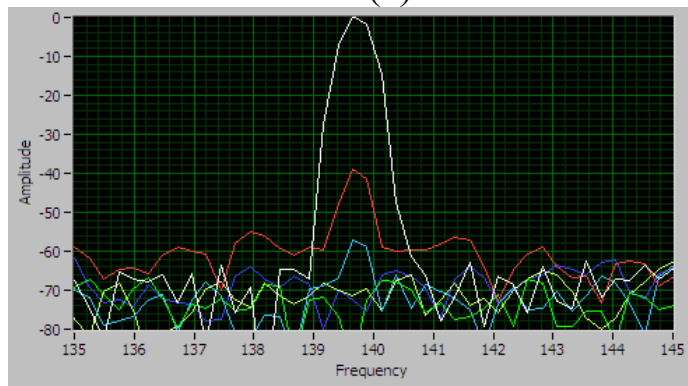

(c)

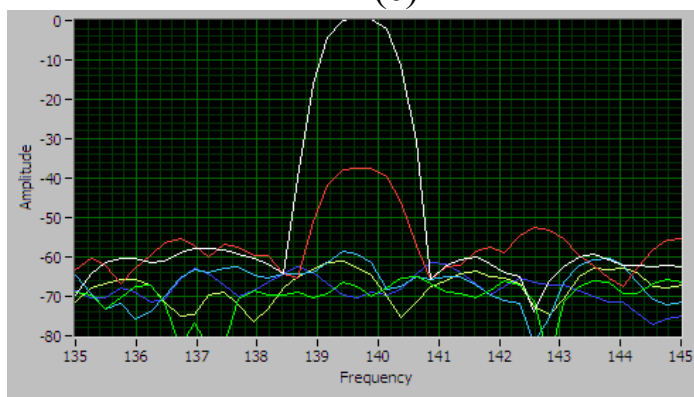

(e)

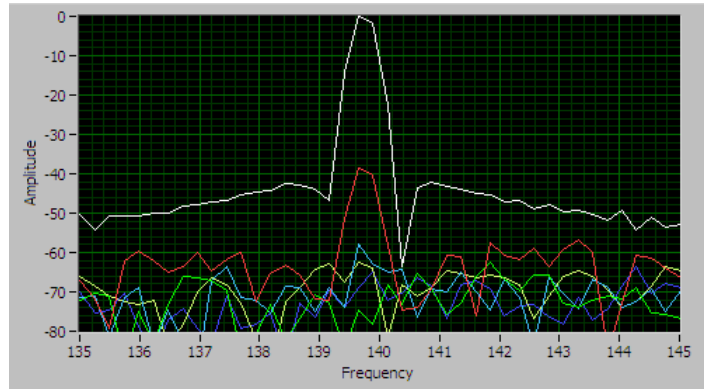

(b)

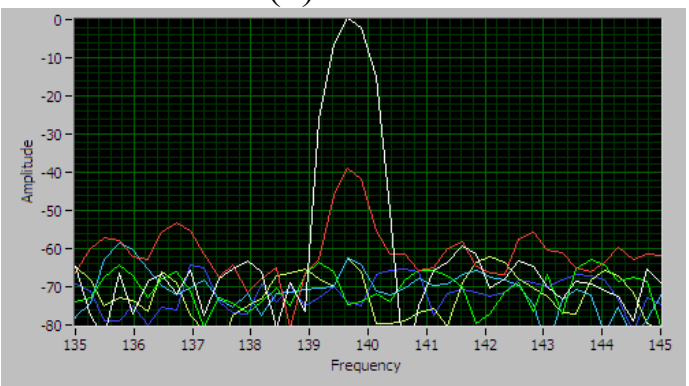

(d)

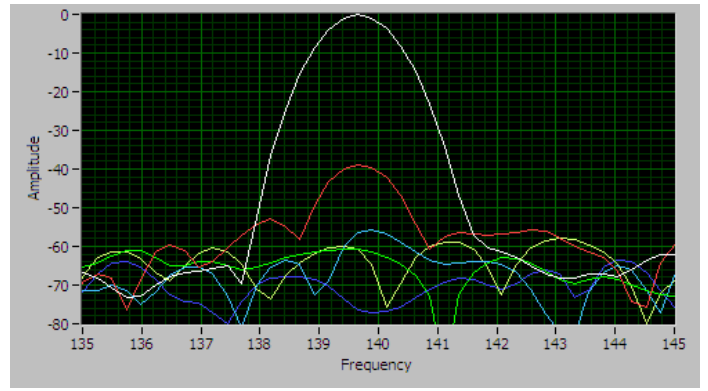

(f)

Figure 5.9 Examples of various windowing techniques can be performed (a) Hanning (b) Hamming (c) Exact-Blackman (d) Blackman-Harris (e) Flat-Top, and (f) Kaiser.

Table 5.4 The comparison of the width windows at certain amplitude

\begin{tabular}{|l|c|c|c|}
\hline \multirow{2}{*}{\multicolumn{1}{|c}{ Window }} & \multicolumn{3}{|c|}{ Width at amplitude (Hz) at } \\
\cline { 2 - 4 } & $-3 \mathrm{~dB}$ & $-6 \mathrm{~dB}$ & $-40 \mathrm{~dB}$ \\
\hline Hanning & 0.330 & 0.431 & 1.600 \\
\hline Hamming & 0.319 & 0.395 & 1.060 \\
\hline Exact-Blackman & 0.370 & 0.527 & 1.252 \\
\hline Blackman-Harris & 0.378 & 0.442 & 1.210 \\
\hline Flat-Top & 0.863 & 1.027 & 2.012 \\
\hline Kaiser & 0.867 & 1.210 & 3.107 \\
\hline
\end{tabular}

Figure 5.9 shows that smooth windowing methods can be implemented in the design of the CompactRIO condition monitoring system. The signal processing tasks 
offer various smooth windowing methods to ease frequency analysis. As stated earlier in Chapter 4, that the main task of windowing is reducing spectral leakage.

In the case of improving spectral resolution, slim frequency peaks are required in FFT-based monitoring applications. The slimness of a peak frequency is determined by the width at half-maximum of the amplitude of a peak frequency, known as fullwidth-half-max. In this research, the amplitude is around $-40 \mathrm{~dB}$. Another convention [124] also defines how slim a main lobe or a peak of frequency is with the width at -3 $\mathrm{dB}$ or $-6 \mathrm{~dB}$. The comparison of width at several amplitudes is presented in Table 5.4. The comparison was taken under normalized frequency.

The slim peaks offer fine separation between two closely spaced frequencies. This slimness is highly desirable when an interaction of a fundamental frequency and many sidebands occurs, such as illustrated in Section 3.2. The spectral resolution improvement can be done not only with other frequencies with similar amplitude but also with other frequencies lower than the main lobe. Table 5.4 shows that the Hanning and Hamming windows are relatively narrow around $-3 \mathrm{~dB}$ and $-6 \mathrm{~dB}$. Furthermore, at amplitude $-40 \mathrm{~dB}$, the Hamming and Blackman-Harris windows have a narrow peak frequency compared with the others. Based on these measurements, the slim peak frequencies can be achieved using the Hamming or Blackman-Harris windows.

In cases where the severity level needs to be examined carefully and periodically, such as for this research, window type with wide peak or amplitude accuracy should be considered. The wide peak results can be obtained through the Flat-top or Kaiser windows, as shown in Table 5.4. However, the high accuracy of amplitude consequently decreases the spectral resolution, as indicated by the wide frequency of the Flat-top or Kaiser windows at $-40 \mathrm{~dB}$. Therefore, if a combination of slimness and amplitude accuracy is required, balanced width at $-3 \mathrm{~dB},-6 \mathrm{~dB}$ and -40 $\mathrm{dB}$ should be considered. This combination meets the characteristics of the BlackmanHarris or Exact-Blackman window. In this research, the Blackman-Harris window was employed as the smoothing window during the fault frequency analysis.

It can be summarized that a brief evaluation of frequency-based signal processing is ready to handle possibilities of rich harmonics and sidebands which may be present due to simultaneous implementation of various monitoring techniques and sensors. However, for more detailed analysis which covers the specific justification of 
each symptom, further improvement is still required in this field. The next section will discuss the experimental evaluation when several faults are introduced.

\subsection{Induction Motor Fault Evaluation}

The induction motors used in the experiments have the following specifications: $2.2 \mathrm{~kW}, 415 \mathrm{~V}, 4.8 \mathrm{~A}$, and 4 poles. Single and multiple real faults were introduced in these induction motors. These faults were broken rotor bar, eccentricity, stator shorted turn, and soft foot. CompactRIO condition monitoring system was used to identify these faults. To display the spectrum of fault frequency analysis of the real time software, CompactRIO-based system was connected to a personal computer. During the tests, the measurement was set to sampling frequency $2000 \mathrm{~Hz}$ and 4096 samples for each channel.

To benchmark the performance of the developed CompactRIO-based system, an additional test was performed. In this test, an identical signal was captured, analyzed and compared using a 12-bit, $200 \mathrm{KS} / \mathrm{s}$ data acquisition card (NI-PCI6024E).

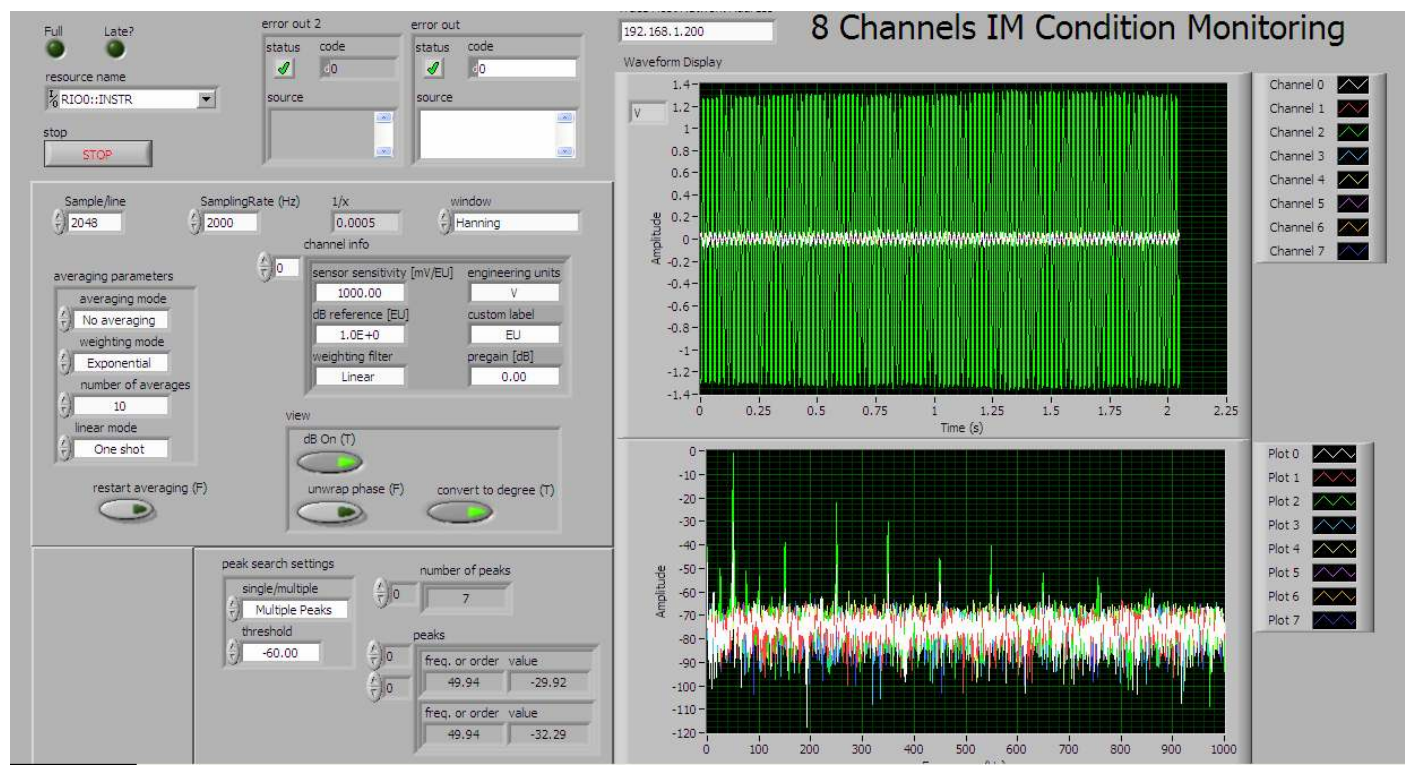

Figure 5.10 User interface of the CompactRIO based condition monitoring system

Figure 5.10 shows the LabVIEW front panel of the developed CompactRIObased condition monitoring system. The figure illustrates the single phase motor current and corresponding frequency spectrum. The top waveform illustrates a time domain waveform, and the bottom graph illustrates a frequency domain waveform. It 
should be noted that in this research the severity level analysis was implemented at only one level limit masking as will be discussed in this section.

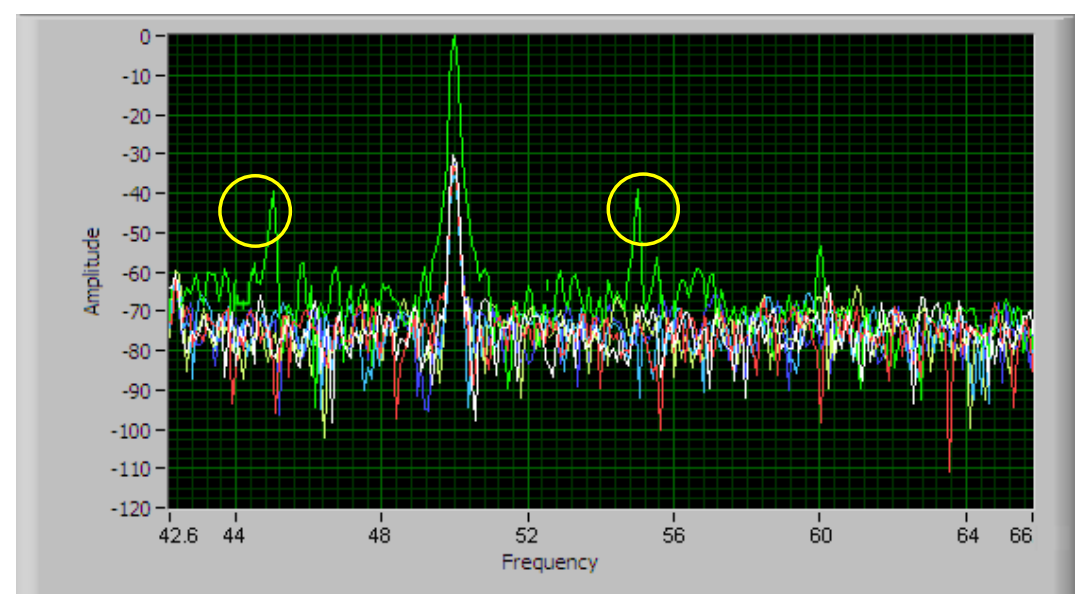

Figure 5.11 A zoomed section of the frequency spectrum indicating the sidebands in circles, under a broken rotor bar fault

In Figure 5.11 a zoomed section of the frequency spectrum is given to identify the broken rotor bar sidebands of $(1 \pm 2 \mathrm{~s}) F_{L}$ in Equations 3.16 and 3.17. This frequency was captured when the motor was fully loaded with slip 0.046 and one broken rotor bar, using stator current monitoring. It can be seen in the figure that the sideband frequencies are located around $45.4 \mathrm{~Hz}$ and $54.4 \mathrm{~Hz}$. The amplitude of these frequencies is around $-40 \mathrm{~dB}$. These frequencies and amplitude can be interpreted as the first harmonic of fault frequency of a broken rotor bar. This result agrees with a previous study in [125] that the sideband frequency around $45 \mathrm{~Hz}$ with amplitude -40 $\mathrm{dB}$ can be an indicator of one broken rotor bar in four poles of induction motors. In other cases of fault, the sideband-related presentation of fault frequency is shown in Figure 5.12.

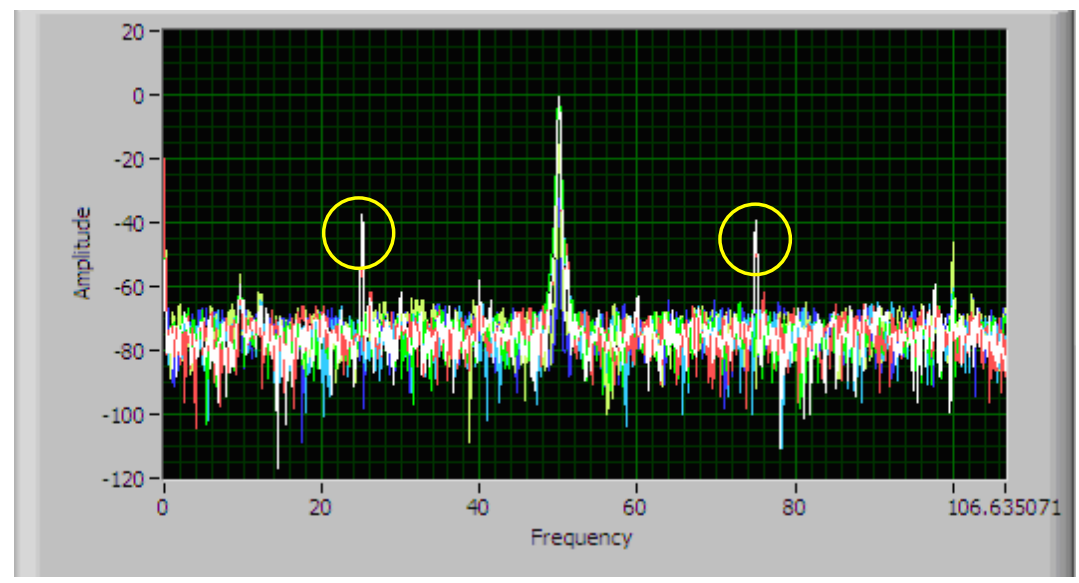

Figure 5.12 A zoomed section of the stator current frequency spectrum indicating the sidebands in circles, under an eccentricity 
Eccentricity-related fault is illustrated in Figure 5.12. This figure shows sidebands of eccentricity fault frequency. The eccentricity type may be mix eccentricity. These frequencies were captured using stator current monitoring when the motor was operated under light load. The figure presents the sidebands around 25 $\mathrm{Hz}$ and $75 \mathrm{~Hz}$, with amplitude around $-42 \mathrm{~dB}$. Based on fault frequency calculation using Equation 3.22, these frequencies are the first sidebands fault frequency or $k=1$ for eccentricity fault. The amplitude also corresponds with a study demonstrating that the amplitude of eccentricity using stator current monitoring is around $-40 \mathrm{~dB}$ in light load [51]. Another fault is shown in Figure 5.13 below.

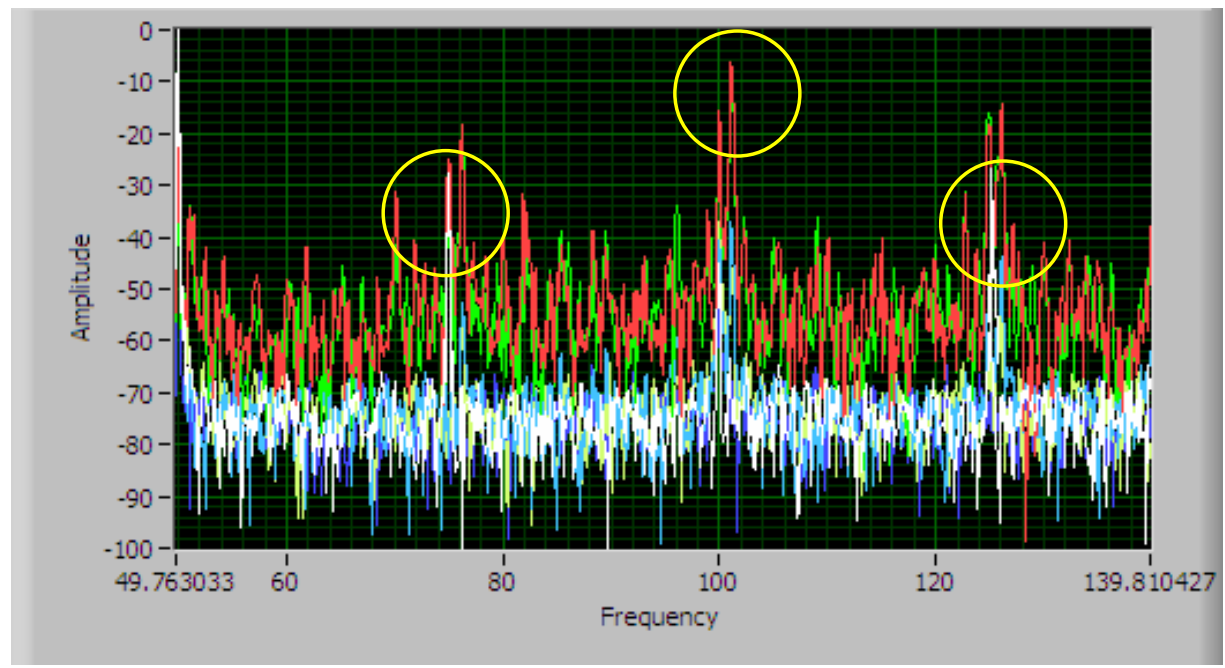

Figure 5.13 A zoomed section of the frequency spectrum of flux leakage and vibration under a shorted turn

Figure 5.13 illustrates the fault frequency under an interturn-related fault. The fault was introduced by creating a short between coils of the same phase under light load. The frequencies were monitored using flux leakage signals (white graph) and vibration signals (red and green graph). Based on the prediction of fault frequency using Equation 3.14, the fault frequency of a shorted turn stator can be located at 74.6 $\mathrm{Hz}$ and $124.6 \mathrm{~Hz}$ for $n=1$ as shown in Figure 5.13 in the left and right circle using flux leakage monitoring. It is also found that the amplitudes of these peaks are around -28 dB. Because the $74.6 \mathrm{~Hz}$ can be interfered with an eccentricity fault frequency under light load, the vibration signals can be use to confirmed this fault frequency. Using vibration monitoring, the shorted turn fault frequency is indicated by $2 F_{L}$, or $100 \mathrm{~Hz}$. This frequency appears in the middle circle, and includes the sidebands. Considering the frequency and amplitude of the stator current and vibration signals, it is clear that a 
shorted turn fault can be identified by the CompactRIO based condition monitoring system.

The results emphasize the performance of the system in identifying fault frequency of an induction motor. The figures also shows that the system is able to differentiate frequencies which are in close proximity to each other. This capability is demonstrated in the sideband detection cases discussed previously. In other words, the frequency resolution setting is adequate for use in fault analysis.

In order to show the system capabilities of the CompactRIO condition monitoring system facing multiple faults and multiple monitoring techniques, a further experimental evaluation was carried out. Figure 5.14 and Figure 5.15 are given to demonstrate the performance of the system under multiple analog signal inputs that were captured simultaneously.

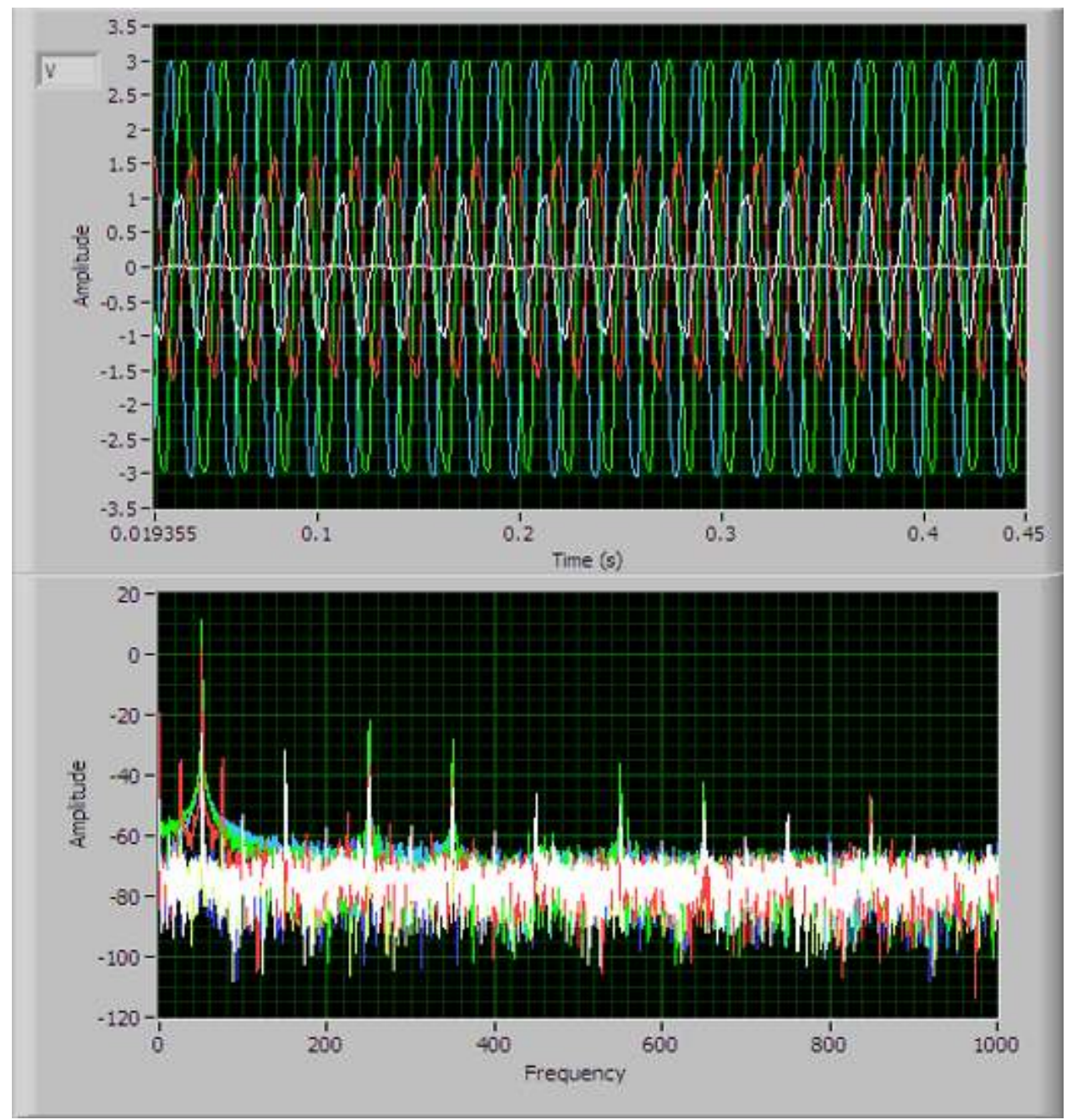

Figure 5.14 Real-time current/voltage waveforms and their frequency spectra under eccentricity fault 
Figure 5.14 illustrates both real current and voltage waveforms, and corresponding frequency spectrums obtained while the motor had an eccentricity fault. It should be noted that the voltage waveform is used primarily to determine the fundamental frequency of power supply or slip. In addition, the voltage signals are not normalized to the fundamental line frequency $50 \mathrm{~Hz}$. In Figure 5.14, the zoom of the graph in the top figure shows that voltage signals and the stator current signals can be displayed in one graph. The FFT frequency of voltage signals is presented in the bottom figure. The figure also points out that rich harmonics can be captured during the measurement. The high harmonic detection is important to confirm any fault frequencies, while the low harmonics may be interfered with other fundamental frequencies.

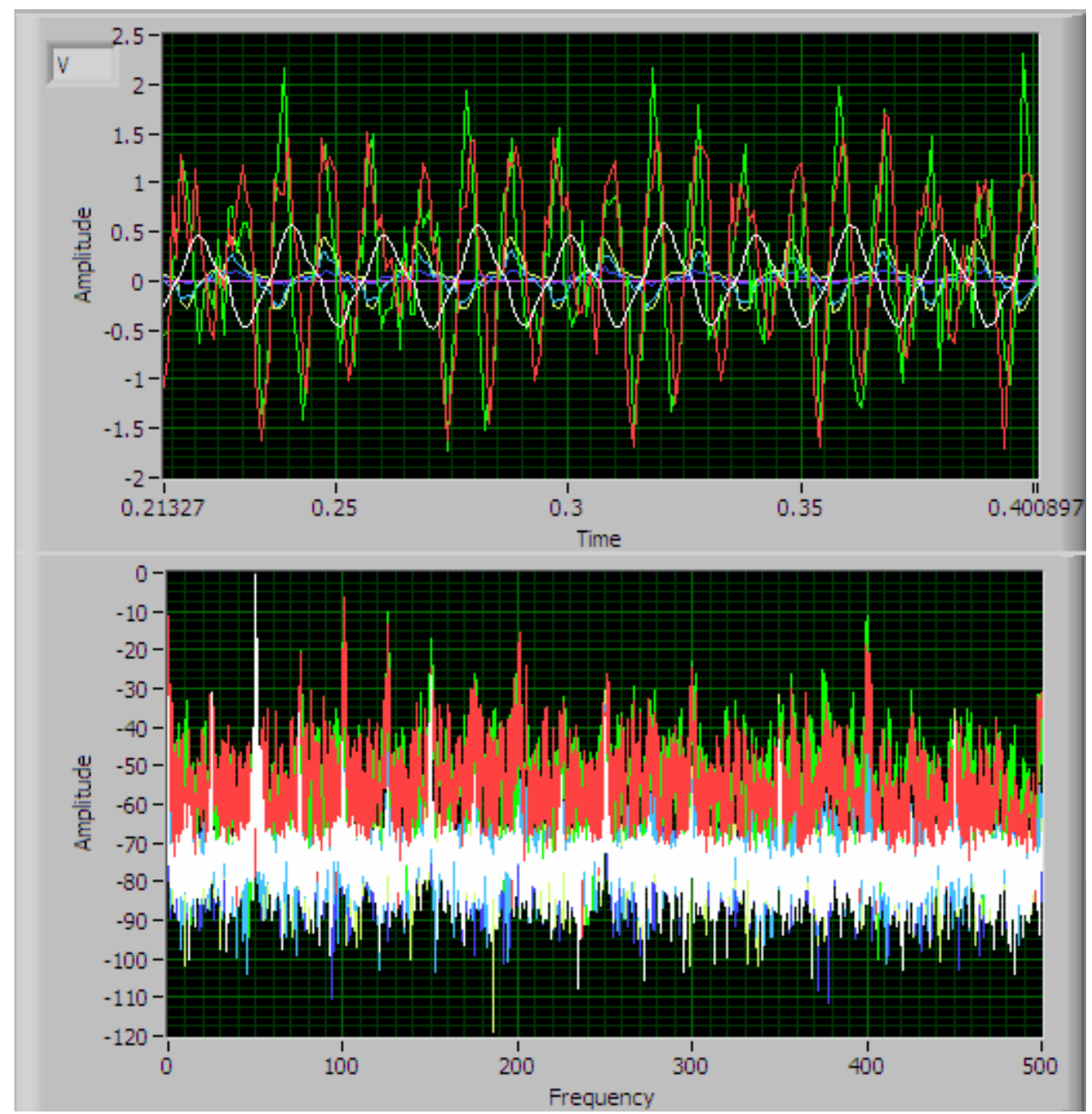

Figure 5.15 Zoom of real-time three axis vibration and axial flux leakage signals with corresponding zoom of frequency spectra under a multiple fault (eccentricity and soft foot) 
In Figure 5.15, a multiple fault (eccentricity and soft foot) was introduced and three vibration signals, and one axial leakage flux of the test motor, were measured. The same monitoring signals were also utilized under a shorted turn stator as discussed previously. The measurement demonstrates the performance of the system when different monitoring techniques are implemented at the same time. As seen in this figure, the software development platform was capable of handling various engineering units and was flexible enough to adjust sensor sensitivities. This capability is demonstrated by the implementation of different conversion units and normalization between flux leakage and vibration monitoring shown in Figure 5.15. This feature allows for an accurate analysis of the measured signals and their separation for future processing. As can be seen in the frequency spectrum of Figure 5.15 , several test set-ups related to fault frequency peaks can be detected by the vibration sensors, and the rotor and stator fault frequency peaks can be identified using the axial leakage flux sensor. It should be noted that for the purposes of this research, bearing faults were not be tested because of equipment limitations.

A repeatability test was also performed for the above given faults. During these tests, the motor with the subject fault ran continuously, and the corresponding sensory signals were captured and analyzed in the CompactRIO based system.

Table 5.5 Performance of peak detection under twenty consecutive tests

\begin{tabular}{|l|c|}
\hline \multicolumn{1}{|c|}{ Faults } & Correct Detection \\
\hline Stator related faults & $75 \%$ \\
\hline Rotor related faults & $85 \%$ \\
\hline Eccentricity related faults & $65 \%$ \\
\hline Set-up faults & $90 \%$ \\
\hline
\end{tabular}

Table 5.5 demonstrates the capability of the system, which is based on twenty consecutive measurements. The measurements are focused on specific fault frequencies of stator, rotor, eccentricity and set-up faults. Most measurement were performed under light load except for the broken rotor bar. This exception was taken following a suggestion in [51]. In this test, the fault frequency selection is performed according to previous discussion. For example, the X-axis threshold of the broken rotor bar frequency was set at the first upper and lower sidebands, i.e. $45.4 \mathrm{~Hz}$ and 
54.6 Hz. The eccentricity was around $25.4 \mathrm{~Hz}$ and $74.6 \mathrm{~Hz}$, and the stator was fixed at $74.6 \mathrm{~Hz}$, and $124.6 \mathrm{~Hz}$, while the vibration signals at $2 F_{L}$ were confirmed. However, it should be noted that the harmonics of these frequencies were also involved to create an array of threshold, as explained in Section 4.5.3.3. In case of set-up related fault frequencies, the threshold was placed at $F_{R}$ and up to its sixth harmonics. In this test, the threshold of peak detections was placed at only one level of limit masking. The lower limit of $\mathrm{Y}$-axis of each fault frequency was placed at around $-5 \mathrm{~dB}$ lower than current amplitude. This technique was carried out only to show how the system operated. In real implementation, specific severity levels, such as bad level, warning level and acceptable level, can be implemented through this monitoring system. These levels can be determined through baseline studies or using other information from the database of a specific induction motor.

As shown in Table 5.5, the higher percentage value corresponds to the level of correct detection of the peaks, which is related to the specific fault frequencies under given fault groups. The set-up fault may be the easiest to identify due to the number of harmonics produced, and several fault contributors such as unbalance, looseness, or misalignment. Other faults can also be easily recognized using this technique. However, this technique requires a large number of databases. This technique can be more difficult when the load variations are involved. According to a study [4], several sidebands of fault frequencies also exist in healthy machines. Therefore, the limit masking should be fixed accurately, especially to deal with small differences of amplitude between sidebands of healthy and unhealthy motors. For example, the detection difficulty occurs under an eccentricity fault such as shown in Table 5.5 where the limit detection sometimes failed to recognize the severity level of this fault frequency. Furthermore, the limit masking can be more complicated when a large number of harmonics is included to gain accurate result.

Despite several limitations, the results presented have pointed out that the CompactRIO condition monitoring system is able to determine faults in induction motors. However, for commercial implementation this development and the results may not be sufficient to make an accurate decision about the condition of a motor, Therefore, further research needs to be conducted for accurate fault frequency analysis including advanced fault recognition, and analysis of multiple faults under load variation. 


\subsection{Conclusion}

In this chapter, the real-time on-site condition monitoring system, based on the CompactRIO architecture mentioned in Chapter 4, was examined. The experimental evaluations cover execution time of the CompactRIO based condition monitoring application, monitoring of CompactRIO hardware resources during the operation, implementation of signal processing frequency analysis, and evaluation of the capability of the CompactRIO monitoring system when various multiple faults are introduced.

As demonstrated in the first section, limit testing VIs may need significant execution times compared with other steps. This in turn may lead to significant consumption of the resources of the CompactRIO real-time host controller. However, the CompactRIO generally is still acceptable for use in condition monitoring.

The CompactRIO based condition monitoring system also has been proved to be capable of detecting single or multiple faults in induction motors. The CompactRIO based monitoring unit, which is fitted with FFT based signal processing and windowing, as described in this chapter, is capable of determining peaks of fault frequencies. These fault frequencies include broken rotor bar, eccentricity fault, stator shorted turn, and set-up related faults. These detected peaks are then monitored using certain customized thresholds to determine severity level. In this research, this severity level is implemented only for single level of limit masking. The results of consecutive measurements highlight the capability of the system to recognize certain faults.

Although these initial results may not be sufficient to make a decisive decision about the unit under test, further investigations are planned using various advanced decision-making algorithms and multiple sensor signals, to identify single or multiple faults. In addition, it would be better if the limit masking detections were also implemented for three severity levels based on the accurate information of the induction motor severity levels from baseline studies or certain standard. 


\section{Chapter 6}

\section{General Conclusions and Suggestions}

\subsection{Conclusions}

The condition monitoring of induction motors is essentially important to maintain safety and reliability during the motor's operation. By implementing condition monitoring strategies, any faults can be identified as early as possible, preventing further catastrophic failures. These failures in turn may lead to unplanned downtime and a lost of production.

This monitoring is usually performed under a condition monitoring system, which incorporates faults of the induction motor, monitoring techniques, sensors and parameters, data acquisition and processing devices, data analysis techniques, and review of the results. These factors may determine how successfully condition monitoring meets a certain objective or monitoring strategy.

Regarding the condition monitoring devices, a common practice in condition monitoring includes the use of mobile and portable devices. These devices are more popular than permanent on-line devices because of price and flexibility. However, these types of devices need an experienced operator to determine the faults. In addition, the periodic inspection carried out using these devices may be too late to determine the induction motor faults.

Therefore, this thesis has proposed the development of a monitoring system that not only has a compact form but also has a function similar to permanent on-line devices. This proposed system might be located next to the motors, is able to perform continuous monitoring, and is equipped with a LEDs indicator to indicate certain faults and severity level of faults. This system was developed based on the CompactRIO (Compact Reconfigurable Input Output) architecture. In this thesis, this system has developed considering several factors, such as: 
1. Considering a group of faults or common faults in induction motors

2. Implementing multiple monitoring techniques to obtain comprehensive results regarding the condition of induction motors

3. Performing signal processing based feature extraction

4. The result or status of induction motors can be understood by less experienced operator

In this thesis, the development of the CompactRIO based monitoring system adopting the criteria mentioned above is detailed. This thesis covers the knowledge and steps necessary for the development of a CompactRIO unit, monitoring software, and the implementation into a condition monitoring system. These detailed developments are discussed through each chapter, and are concluded in the following paragraphs.

In Chapter 2, a comprehensive literature review was given in order to provide an understanding of current studies related to condition monitoring. This review involves:

1. statistical studies of induction motor faults

2. various monitoring techniques which emphasize multi-purposes on-line condition monitoring techniques

3. data analysis techniques especially FFT based signal processing

4. current commercial condition monitoring devices

5. studies on low-cost condition monitoring devices and

6. various CompactRIO applications in industry and research.

It was pointed out in this chapter that the CompactRIO based condition monitoring system should have features such as:

1. Multiple fault detections, for example bearing-related faults, stator-related faults, rotor-related faults, eccentricity faults, mechanical set-up faults, and voltage problems

2. Multiple monitoring techniques: vibration monitoring, stator current, stator voltage, and flux leakage monitoring

3. FFT based signal-processing analysis to ease fault frequency analysis

4. LEDs indicator to indicate the severity level of each fault of the induction motor. 
In chapter 3, induction motor theory and operation principles were discussed. This discussion provided important background understanding for further discussion of fault frequency predictors. These various fault frequency predictors were explored in this chapter to incorporate a wide range of possibilities in order to detect each group of faults. The fault frequency predictor covered all faults indicated in the previous chapter, according to each monitoring technique. The frequency predictors summarized in this chapter were then implemented in the development of the CompactRIO monitoring unit.

In chapter 4, the CompactRIO based condition monitoring development was explained. This chapter provided information on the CompactRIO based unit, the general architecture, and the CompactRIO custom Module Development Kit. In addition, the complete CompactRIO segmented development, both under LabVIEW FPGA and real-time environment, was discussed. The custom-made CompactRIO indicator unit was also demonstrated. It was concluded that the CompactRIO development has followed the necessary requirements for achieving maximum performance and analysis results.

Finally, CompactRIO performances were demonstrated in Chapter 5. The performance evaluation covered execution time evaluation, real-time hardware resources, FFT signal processing evaluation, and single or multiple real faults of induction motors. It was pointed out that the condition monitoring system has reasonable real-time performance for condition monitoring. However, specific limit testing needs further improvement to reduce the execution time, and to save CompactRIO real-time resources. Further, signal processing was demonstrated to separate two closely spaced peak frequencies. In addition, a windowing technique can be implemented to improve this capability. Through this evaluation, it was highlighted that the maximum FFT resolution can be achieved for multi channels is only 4096 lines. However, this limitation does not compromise the effectiveness of the early warning condition monitoring system. Finally, the CompactRIO performances when real faults were introduced were given. The CompactRIO based monitoring system is able to determine single and multiple faults using multiple monitoring techniques. It was also underlined that decision-making algorithms need further improvement in order to gain more accurate results than currently had.

It can be concluded from the discussion presented that the CompactRIO based condition monitoring system is able to be implemented in a real environment as an on- 
site real-time unit. It is also able to perform continuous measurement, and display the results effectively. In other words, it is suitable for early warning system where the general condition of an induction motor needs to be examined continuously.

\subsection{Suggestions}

As concluded in this thesis, overall, the system is able to perform condition monitoring of induction motor. However, for further implementation in industry, many areas should be improved. It is suggested that more algorithms of signal processing and fault analysis can be developed under FPGA. This method might reduce the resources of CompactRIO real-time host controller, which in turn will reduce total execution time.

In addition, other research in the development of decision-making algorithms should be carried out not only to improve the accuracy of results but also to reduce total execution time. This research can be numerical, statistical, or artificial intelligence based decision-making. For eight channel monitoring signals and multiple faults, these algorithms need to be implemented in highly efficient, reliable, fast, and simple VIs.

Finally, the CompactRIO system has a versatile capacity to be expanded in such a way where user's application is easy to be developed. Therefore, the CompactRIO architecture can be utilized not only to measure one motor but possibly a group of motors. To improve the signal processing capabilities for a such configuration, a dedicated signal-processing and decision-making host server can be used to take over the current task of the CompactRIO real-time host controller, through distributed network processing. 


\section{References}

[1] _ , "Electric machines • Mighty motors", Siemens AG, 2006, [Online], Available:

http://w1.siemens.com/innovation/en/publikationen/publications_pof/pof_sprin g_2006.htm

[2] _ , "Induction motor design". Myanmar, 2007, [Online], Available: http://bp0.blogger.com/_PCuPZfbcyH0/RxAaAP7C_7I/AAAAAAAAAKo/2V Jnnor83E8/s1600-h/imd+pic+\%281\%29.JPG

[3] B. S. Payne, B. Liang, A. D. Ball, "Modern condition monitoring technique for rlectrical machine", presented at $I^{\text {st }}$ International Conference on the Integration of Dynamics, Monitoring and Control, Manchester, UK, 1999.

[4] W. T. Thomson, "A review of on-line condition monitoring techniques for three phase squirrel induction motors - past, present and future", presented at Proc. IEEE Int. Symp. Diagnostics Electrical Machines, Power Electronics Drives, Gijon, Spain, 1999.

[5] H. W. Penrose, "Test methods for determining the impact of motor condition on motor efficiency and reliability", ALL-TEST Pro, LLC, Old Saybrook, USA, [Online], Available:

http://www.alltestpro.com/documents/Testmethodsfordetermingtheimpactofm otorconditiononmotorefficiency.pdf

[6] S. J. Mitchell, "From vibration measurements to condition based maintenance, seventy years of continuous progress", Sound and Vibration Magazine, vol. 41, pp. 62-75, 2007.

[7] P. Girdhar, and C. Scheffer, Practical machinery vibration analysis and predictive maintenance, First ed. Burlington: Newnes, Elsevier, 2004.

[8] S. A. Ansari, and R. Baig, "A PC-based vibration analyzer for condition monitoring of process machinery", IEEE Trans. Instrum. Meas., vol. 47, pp. 378-383, 1998.

[9] J. Deery, "The real history of real-time spectrum analyzers, a 50-year trip down memory lane", Sound and Vibration Magazine, vol. 41, pp. 54-59, 2007.

[10] S. Welaratna, "Thirty years of FFT analyzers ..." Sound and Vibration Magazine, pp. 1-5, 1997.

[11] G. Betta, C. Liguori, and A. Pietrosanto, "A multi-application FFT analyzer based on a DSP architecture", IEEE Trans. Instrum. Meas., vol. 50, pp. 825832, 2001.

[12] G. Betta, C. Liguori, A. Paolillo, and A. Pietrosanto, "A DSP-based FFTanalyzer for the fault diagnosis of rotating machine based on vibration analysis", IEEE Trans. Instrum. Meas., vol. 51, pp. 1316-1322, 2002.

[13] B. S. Payne, A. D. Ball, M. Husband, B. Simmers, and F. Gu, "An approach to the development of condition monitoring for a new machine by example", presented at International conference on Power, electronic, machines and drives, 2002.

[14] O. V. Thorsen, and M. Dalva, "Failure identification and analysis for highvoltage induction motors in the petrochemical industry", IEEE Trans. Ind. Appl., vol. 35, pp. 810 - 818, 1999. 
[15] A. K. S. Jardine, D. Lin, and D. Banjevic, "A review on machinery diagnostics and prognostics implementing condition-based maintenance", Mechanical Systems and Signal Processing, vol. 20, pp. 1483-1510, 2006.

[16] P. T. Tavner, and J. Penman, Condition monitoring of electrical machines. Letchworth, England: Research Studies Press LTD, John Wiley and Sons Inc, 1987.

[17] G. K. Singh, and S. A. S. Al Kazzaz, "Induction machine drive condition monitoring and diagnostic research-a survey", Electric Power Systems Research, vol. 64, pp. 45-158, 2003.

[18] B. Mirafzal, and N.A.O. Demerdash, "On innovative methods of induction motor interturn and broken-bar fault diagnostics", IEEE Trans. Ind. Appl., vol. 42, pp. 405-414, 2006.

[19] W. T. Thomson, and A. Barbour, "On-line current monitoring and application of a finite element method to predict the level of static airgap eccentricity in three-phase induction motors", IEEE Trans. Energy Convers., vol. 13, pp. 347-354, 1998.

[20] W. T. Thomson, D. Rankin, and D. G. Dorrell,, "On-line current monitoring to diagnose airgap eccentricity in large three-phase induction motors-Industrial case histories verify the predictions", IEEE Trans. Energy Convers., vol. 14, pp. 1372-1378, 1999.

[21] S. Kolla, and L. Varatharasa, "Identifaying three-pase induction motor faults using artificial neural network", ISA Transaction, vol. 39, pp. 433-439, 2000.

[22] A. Siddique, G. S. Yadava, and B. Singh, "A review of stator fault monitoring techniques of induction motors", IEEE Trans. Energy Convers., vol. 20, 2005.

[23] J. Yang, S. B. Lee, J. Yoo, S. Lee, Y. Oh, and C. Choi, "A stator winding insulation condition monitoring technique for inverter-fed machines", IEEE Trans. Power Electron., vol. 22, pp. 2026 - 2033, 2007.

[24] X. Huang, T. G. Habetler, R. G. Harley, and E. J. Wiedenbrug, "Using a surge tester to detect rotor eccentricity faults in induction motors", IEEE Trans. Ind. Appl., vol. 43, pp. 1183 - 1190, 2007.

[25] R. M. Tallam, et al., "A survey of methods for detection of stator-related faults in induction machines", IEEE Trans. Ind. Appl., vol. 43, pp. 920-33, 2007.

[26] W. R. Finley, M. M. Hodowanec, K. S. Hussain, and J. Larabee, "To test or not to test, understanding electrical and mechanical test performed on induction motors", IEEE Ind. Appl. Mag., vol. 10, pp. 167- 178, 2004.

[27] S. Nandi, "Detection of stator faults in induction machines using residual saturation harmonics", IEEE Trans. Ind. Appl., vol. 42, pp. 1201 - 1208, 2006.

[28] J. T. Taylor, The vibration ahandbook, A practical guide for solving rotating machinary problems, First ed. Tampa Florida: Vibration Consultan Inc., 1994.

[29] D. E. Butler, "The shock-pulse method for the detection of damaged rolling bearings", Non-Destructive Testing, vol. 6, pp. 92-95, 1973.

[30] B. S. Payne, A. D. Ball, F. Gu, and W. Li, "Head to head assasement of the relative fault detection and diagnosis capabilities of confentional vibration and airborne acoustic monitoring", presented at Proceeding of 13th International Congress on Condition Monitoring and Diagnostic Engineering Management (COMADEM 2000), Texas, USA, 2000.

[31] N. a. B. C. N. Tandon, "Comparison of vibration and acoustic measurement techniques for the condition monitoring of rolling element bearings", Tribology International, vol. 25, pp. 205-212, 1992. 
[32] M. E.-H. Benbouzid, and G. B. Kliman, "What stator current processing-based technique to use for induction motor rotor faults diagnosis?" IEEE Trans. Energy Convers., vol. 18, pp. 238 - 244, 2003.

[33] A. Bellini, et al., "On-field experience with online diagnosis of large induction motors cage failures using MCSA", IEEE Trans. Ind. Appl., vol. 38, pp. 10451053, 2002.

[34] A. M. Trzynadlowski, and E. Ritchie, "Comparative investigation of diagnostic media for induction motors: A case of rotor cage faults", IEEE Trans. Ind. Electron., vol. 47, pp. 1092 - 1099, 2000.

[35] V. V. Thomas, K. Vasudevan, and V. J. Kumar, "Online cage rotor fault detection using air-gap torque spectra", IEEE Trans. Energy Convers., vol. 18, pp. $265-270,2003$.

[36] S. F. Legowski, et al., "Instantaneous power as a medium for the signature analysis of induction motors", IEEE Trans. Ind. Appl., vol. 32, pp. 904-909, 1996.

[37] Z. Liu, X. Yin, Z. Zhang, D. Chen, and W. Chen, "Online rotor mixed fault diagnosis way based on spectrum analysis of instantaneous power in squirrel cage induction motors", IEEE Trans. Energy Convers., vol. 19, pp. 1060 1069, 2004.

[38] G. Didier, E. Ternisien, O. Caspary, and H. Razik, "Fault detection of broken rotor bars in induction motor using a global fault index", IEEE Trans. Ind. Appl., vol. 42, pp. 79 - 88, 2006.

[39] G. Stone, and J Kapler, "Stator winding monitoring", IEEE Ind. Appl. Mag., vol. 4, pp. 15 - 20, 1998.

[40] M. Arkan, A H. Calis, and M. E. Tagluk, "Bearing and misalignment fault detection in induction motors by using the space vector angular fluctuation signal", Electrical Engineering, vol. 87, 2005.

[41] R. A. Collacott, Vibration monitoring and diagnosis, technique for costeffective plant maintenance. London: George Godwin Limited, John Wiley \& Son, 1979.

[42] B. Li, M-Y Chow, Y.Tipsuwan and J C. Hung, "Neural-network-based motor rolling bearing fault diagnosis", IEEE Trans. Ind. Electron., vol. 47, pp. 984 993, 2000.

[43] R. R. Schoen, T. G. Habetler, F. Kamran, and R. G. Bartheld, "Motor bearing damage detection using stator current monitoring", IEEE Trans.Ind. Appl., vol. 31, pp. 1274-1279, 1995.

[44] L. Eren, and M.J. Devaney, "Bearing damage detection via wavelet packet decomposition of the stator current", IEEE Trans. Instrum. Meas., vol. 53, pp. 431-436, 2004.

[45] J. Zarei, and J. Poshtan, "Bearing fault detection using wavelet packet transform of induction motor stator current", Tribology International, vol. 40, pp. 763-769, 2007.

[46] N. Tandon, G.S. Yadava, and K.M. Ramakrishna, "A comparison of some condition monitoring techniques for the detection of defect in induction motor ball bearings", Mechanical Systems and Signal Processing, vol. 21, pp. 244256, 2007.

[47] A. M. Knight, and Bertani S.P, "Mechanical fault detection in a medium-sized induction motor using stator current monitoring", IEEE Trans. Energy Convers., vol. 20, pp. 753-760, 2005. 
[48] M. F. Cabanas, M.G. Melero, G.A Orcajo, J.R. Faya and J.S Sariego, "Experimental application of axial leakage flux to the detection of rotor asymmetries, mechanical anomalies and interturns short circuits in working induction motors." presented at International Conference on Electrical Machines, 1998.

[49] W. T. Thomson, and M. Fenger, "Current signature analysis to detect induction motor faults", IEEE Ind. Appl. Mag., vol. 7, pp. 26-34, 2001.

[50] W. T. Thomson, and D. Morison, "On-line diagnosis of stator shorted turns in mains and inverter fed low voltage induction motors", presented at International Converence on Power Electronics, Machines and Drives 2002, University of Bath, UK, 2002.

[51] I. Ahmed, R. Supangat, J. Grieger, N. Ertugrul and W. L. Soong, "A baseline study for on-line condition monitoring of induction machines", presented at Australasian Universities Power Engineering Conference (AUPEC 2004), Brisbane, Australia, 2004.

[52] J. Penman, H. G. Sedding, B A Llod and W T Fink, "Detection and location of interturn short circuits in stator windings of operating motors", IEEE Trans. Energy Convers., vol. 9, pp. 652-658, 1994.

[53] J. Penman, M. N. Dey, A. J. Tait, and W. E. Bryan, "Condition monitoring of electrical drives", Proc. Inst. Elect. Eng. B, vol. 133, pp. 142-148, 1986.

[54] W. R. Finley, M. M. Hodowanec, and W. G. Holter, "An analytical approach to solving motor vibration problem", IEEE Trans. Ind. Appl., vol. 36, pp. 1467-1480, 2000.

[55] R. R. Schoen, and T. G. Habetler, "Effects of time-varying loads on rotor fault detection in induction machines", IEEE Trans. Ind. Appl., vol. 31, pp. 900 906, 1993.

[56] G. B. Kliman, R. A. Koegl, J. Stein, R. D. Endicott, and M.W. Madden, "Noninvasive detection of broken rotor bars in operating induction motors", IEEE Trans. Energy Convers., vol. 3, pp. 873-879, 1988.

[57] S. Nandi, H. A. Toliyat, and X. Li, "Condition monitoring and fault diagnosis of electrical motors-A review", IEEE Trans. Energy Convers., vol. 20, pp. 719-729, 2005.

[58] I. Ahmed, N. Ertugrul and W. L. Soong, "A study on the detection of fault frequencies for condition monitoring of induction machines", presented at Australasian Universities Power Engineering Conference (AUPEC 2005), Hobart, Tasmania, Australia, 2005.

[59] H. Meshgin-Kelk, J. Milimonfared, and H. A. Toliyat, "Interbar currents and axial fluxes in healthy and faulty induction motors", IEEE Transaction on Transaction Industry Applications, vol. 40, pp. 128 - 134, 2004.

[60] F. Filippetti, G., Franceschini, and C. Tassoni, "Neural networks aided on-line diagnostic of induction motor rotor faults", IEEE Trans. Ind. Appl., vol. 31, pp. 892 - 899, 1995.

[61] J. H. Jung, J. J. Lee, and B. H. Kwon, "Online diagnosis of induction motors using MCSA", IEEE Trans. Ind. Electron., vol. 53, pp. 1842-1852, 2006.

[62] G. Franceschini, and C. Tassoni, "Time harmonic investigation of flux density in the stator and rotorframe oriented to diagnostics of cage induction machines", presented at Sixth International Conference on Electrical Machines and Drives, Oxford, UK, 1993. 
[63] T. J. Sobczyk, K. Weinreb, and A. Izworski, "Recognition of rotor eccentricity of induction motors based on the fourier spectra of phase currents", presented at International Conference on Electrical Machines, Istanbul, Turkey, 1998.

[64] D. G. Dorrell, W.T. Thomson, and S. Roach, "Analysis of airgap flux, current, and vibration signals as a function of the combination of static and dynamic airgap eccentricity in 3-phase induction motors", IEEE Trans. Ind. Appl., vol. 33, pp. 24 - 34, 1997.

[65] A. Korde, "On-line conditional monitoring of motors using electrical signature analysis", presented at Recent Advances in Contion-Based Plant Maintenance Seminar, Nitie, Mumbai, India, 2002.

[66] T. W. S. Chow, G. Fei, "Three phase induction machines asymmetrical faults identification using bispectrum", IEEE Trans. Energy Convers., vol. 10, pp. 688-693, 1995.

[67] _ _ 4383 Piezoelectric Charge Accelerometer, side connector, cable included", Brüel \& Kjær, 2008, [Online], Available:

http://www.bksv.com/Products/TransducersConditioning/VibrationTransducer s/Accelerometers/4383.aspx

[68] _ "Vikon Vibrationskonsult AB - rotor balancing and condition monitoring", Power-technology.com, 2008, [Online], Available: http://www.power-technology.com/contractors/condition/vikon/vikon4.html

[69] , "Proximity Transducers, Seismic Sensors and Accessories", ProvibTech, 2008, [Online], Available: http://www.provibtech.com/pdf/TM079VD.pdf

[70] C. H. Hansen, Notes for a course in machine condition monitoring and fault diagnosis, vol. 1. Adelaide: University of Adelaide, Department of Mechanical Engineering, 1987. Available: "i1010 AC/DC Current Clamp", Fluke Corporation, 2008, [Online], http://us.fluke.com/usen/products/AccessoryDetail.htm?catalog name=FlukeU nitedStates\&cs id=9323(FlukeProducts)

[74] Y. Han, and Y. H. Song, "Condition monitoring techniques for electrical equipment-A literature survey", IEEE Trans. Power Del., vol. 18, pp. 4-13, 2003.

[75] F. F. Costa, L. A. L. de Almeida, S. R. Naidu, and E. R. Braga-Filho, "Improving the signal data acquisition in condition monitoring of electrical machines", IEEE Trans. Instrum. Meas., vol. 53, pp. 1015-1019, 2004.

[76] N. Ertugrul, "Power quality and condition monitoring- lecture note", University of Adelaide, School of Electrical and Electronic Engineering, 2004.

[77] A. J. M. Cordoso, and E.S Saraiva, "Predicting the level of airgap eccentricity in operating three-phase induction motors, by Park's Vector approach", presented at IEEE Industry Application Society, 1992. , "Machinery Health ${ }^{\text {TM }}$ Management, product data sheet, Model 343 flux coil", Emerson CSI, 2004, [Online], Available: http://www.peabprocess.se/Templates/send file pr.asp? ID $=249$

[79] J. Ramirez-Nino, and A. Pascacio, "Detecting inter-turn short circuits in rotor windings", IEEE Comput. Appl. Power, vol. 14, pp. 39-42, 2001. 
[80] M. E. Benbouzid, "A review of induction motors signature analysis as a medium for faults detection", IEEE Trans. Ind. Electron., vol. 47, pp. 984-993, 2000.

[81] D. M. Yang, A. F. Stronach, P. MacConnell, and J. Penman, "Third-order spectral techniques for the diagnosis of motor bearing condition using artificial neural networks", Mechanical Systems and Signal Processing, vol. 16, pp. 391-411, 2002.

[82] N. Arthur, and J. Penman, "Induction machine condition monitoring with higher order spectra", IEEE Trans. Ind. Electron., vol. 47, pp. 1031 - 1041, 2000.

[83] M. E. Benbouzid, M. Vieira, and C. Theys, "Induction motors' faults detection and localization using stator current advanced signal processing techniques", IEEE Trans. Power Electron., vol. 14, pp. 14-22, 1999.

[84] Z. Ye, B. Wu, and A. Sadeghian, "Current signature analysis of induction motor mechanical faults by wavelet packet decomposition", IEEE Trans. Ind. Electron., vol. 50, pp. 1217 - 1228, 2003.

[85] J. L. Frarey, "Pitfalls in the analysis of machinery vibration measurements", Sound and Vibration Magazine, vol. 36, pp. 18-24, 2002.

[86] X. Zhang, Z. Song, and Z. Yao, "Portable vibration spectrum analyzer", Nios II Embedded Processor Design Contest, Third Prize, J. Xu, and X. Wang, Altera, 2005, [Online], Available: http://www.altera.com/literature/dc/2.82005 China 3rd InstitutePLA-web.pdf

[87] G. Betta, M. D’Apuzzo, C. Liguori, and A. Pietrosanto, "An intelligent FFT analyzer", IEEE Trans. Instrum. Meas., vol. 47, pp. 1173-1179, 1998.

[91] C. Dase, J.S., Falcon, and B. Maccleery, "Motorcycle control prototyping using an FPGA-based embedded control system", IEEE Control. Syst. Mag., vol. 26, pp. 17-21, 2006.

[92] B. Al-Naami, J. Chebil, B. Trabsheh, and H. Mgdob, "Developing custom signal processing algorithm with LabView FPGA and CompactRIO to detect the aortic stenosis disease", Computers in Cardiology, vol. 33, pp. 193-196, 2006.

[93] P. Jeter, I.K. Kim, D. Bever, D. Doll, and C. Jaskolski, "GA Urban Maglev stable levitation, propulsion and guidance", The 19th International Conference on Magnetically Levitated Systems and Linear Drives, incorporating the "6. Dresdner Fachtagung Transrapid. Dresden - Germany, 2006, [Online], Available: http://www.maglev2006.de/095 Jeter/No95 Jeter_ok.pdf

[94] K. Fowler, "Data processing in measurement Instrumentation, Part 9 in a series of tutorials in instrumentation and measurement", IEEE Instrum. \& Meas. Mag., vol. 9, pp. 36-42, 2006. , "Basics of AC motors", Siemens, 2007, [Online], Available: http://www.sea.siemens.com/step/templates/lesson.mason?motors:1:1:1 , "Wound rotor induction motors 4-12 poles, up to $8000 \mathrm{~kW}, 11500 \mathrm{HP}$ ". Switzerland ABB, 2007, [Online], Available: 
http://library.abb.com/global/scot/scot234.nsf/veritydisplay/a53c8284b6e9830 3c22570730028f069/\$File/Wound\%20Rotor\%20Induction\%20Motors.pdf

[97] T. Wildi, Electrical machines drives and power system. Columbus, Ohio: Prentice Hall, 2005.

[98] A. Harnoy, Bearing design in machinery: engineering tribology and lubrication. New York: CRC, Marcel Dekker, Inc., 2003.

[99] W. R. Finley, and M.M. Hodowanec, "Sleeve versus antifriction bearings: Selection of the optimal bearing for induction motors", IEEE Trans. Ind. Appl., vol. 38, pp. 909 - 920, 2002.

[100] D. Bogh, J.R. Crowell, and D. Stark, "Bearings for IEEE 841 motors", IEEE Trans. Ind. Appl., vol. 39, pp. 1578 - 1583, 2003.

[101] D. Mba, and Raj B. K. N. Rao, "Development of acoustic emission technology for condition monitoring and diagnosis of rotating machines: bearings, pumps, gearboxes, engines, and rotating structures", The Shock and Vibration Digest,, vol. 38, pp. 3-16, 2006.

[102] W. R. Finley, "Troubleshooting induction motors", presented at Industry Applications Conference, Rome, Italy, 2000.

[103] R. L. Schiltz, "Forcing frequency identification of rolling element bearings", Sound and Vibration Magazine, vol. May, pp. 16-19, 1990.

[104] G. J. Paoletti, and A. Rose, "Improving existing motor protection for medium voltage motors", IEEE Trans. Ind. Appl., vol. 25, pp. 456 - 464, 1989.

[105] W. R. Finley, and M. M. Hodowanec, "Selection of copper vs aluminium rotors for inducton motors", presented at 47th Annual Petroleum and Chemical Industry Conference, 2000.

[106] J. Milimonfared, H. M. Kelk, S. Nandi, A. D. Minassians, and H. A. Toliyat, "A novel approach for broken-rotor-bar detection in cage induction motors", IEEE Trans. Ind. Appl., vol. 35, pp. 1000-1006, 1999.

[107] N. M. Elkasabgy, A. R. Eastham, and G. E. Dawson, "Detection of broken bars in the cage rotor on an induction machine", IEEE Trans. Ind. Appl., vol. 28, pp. 165-171, 1992.

[108] F. Filippetti, G. Franceschini, C. Tassoni, and P. Vas, "AI techniques in induction machines diagnosis including the speed ripple effect", IEEE Trans. Ind. Appl., vol. 34, pp. 98-108, 1998.

[109] S. Nandi, S. Ahmed, and H. A. Toliyat, "Detection of rotor slot and other eccentricity related harmonics in a three phase induction motor with different rotor cages", IEEE Trans. Energy Convers., vol. 16, pp. 253-260, 2001.

[110] S. J. Reimann, "Balancing considerations for high-speed machining", Gardner Publications, Inc, 2008, [Online], Available: http://www.moldmakingtechnology.com/articles/120201.html

[111] N. Mathur, "NI CompactRIO - Reconfigurable control and acquisition system", National Instrument, 2006, [Online], Available: http://zone.ni.com/devzone/cda/tut/p/id/2856

[112] , "CompactRIO product selection guide", National Instruments Corporation, 2008, [Online], Available: http://zone.ni.com/devzone/cda/tut/p/id/3114

[113] , "CompactRIO I/O module development kit user manual". Austin, Texas, USA National Instruments Corp., 2004.

[114] _ _ "Operating instructions, NI 9201/9221, 8-channel, 12-Bit analog input modules". Austin, Texas, USA National Instruments Corp., 2007, [Online], Available: http://www.ni.com/pdf/manuals/373783f.pdf 
Semiconductor, Semiconductor Components Industries, 2004, [Online], Available: http://www.onsemi.com/pub/Collateral/NLSF595-D.PDF , "N500TGLR2D super bicolor lamp". Brooklyn, New York LC LED

Corporation, 2008, [Online], Available: http://www.lc-

led.com/products/n500tglr2d.html

[117] , "Developing digital communication interfaces with LabVIEW FPGA (Part 1)", National Instrument Corp., 2007, [Online], Available:

http://zone.ni.com/devzone/cda/tut/p/id/5385

[118] _ ,LabVIEW FPGA module 8.2 help", National Instrument Corp., 2006, [Online], Available: http://zone.ni.com/reference/en-XX/help/371599B-01/

[119] _ ,LabVIEW, Digital filter design toolkit user manual". Austin, Texas

National Instruments Corp., 2005, [Online], Available:

http://digital.ni.com/manuals.nsf/websearch/A685C04E4EF714E986256F8000 72D9C5

[120] , "Digital filter design toolkit", National Instrument Corp., 2006, [Online], Available:

http://digital.ni.com/manuals.nsf/websearch/BAF1F997647739F08625725E00 $\underline{0 \mathrm{C} 1 \mathrm{~F} 57}$

[121] _ "Developing a hardware-timed batch process system with CompactRIO", National Instrument Corp., 2006, [Online], Available: http://zone.ni.com/devzone/cda/tut/p/id/3249

[122] _ "LabVIEW FPGA module training", National Instrument Corp., 2007, [Online], Available: http://zone.ni.com/devzone/cda/tut/p/id/3555

[123] R. B. Randall, Frequency analysis. Denmark: Brüel kjær, 1987.

[124] _ ,LabVIEW 8.2 help", National Instrument Corp., 2006, [Online], Available:

http://digital.ni.com/manuals.nsf/websearch/88FCF2840C4C7A1F862571D20 06Е0A94

[125] J. Siau, A. Graff, W. Soong and N. Ertugrul, "Broken bar detection in induction motors using current and flux spectral analysis", presented at Australasian Universities Power Engineering Conference 2003, Christchurch, New Zealand, 2003.

[126] _ , "SVFA spectrum peak search (sound and vibration measurement suite), sound and vibration measurement suite 6.0 help", National Instrument Corp., 2007, [Online], Available: http://zone.ni.com/reference/en-XX/help/372416A01/svlshrd/spectrum peak_search/

[127] _ "LabVIEW execution trace toolkit user guide", National Instrument Corp., 2004, [Online], Available: http://www.ni.com/pdf/manuals/323738a.pdf

[128] _ , "LabVIEW help, Real-time execution trace toolkit", National

Instrument Corp., 2007, [Online], Available:

http://www.ni.com/pdf/manuals/370941b.zip 


\section{Appendix}

\section{A.1 The Details PCB of CompactRIO indicator module}

Some details of custom made CompactRIO indicator module were explained in Chapter 4. This includes schematic diagram, components and final design. The PCB development is shown in this appendix.

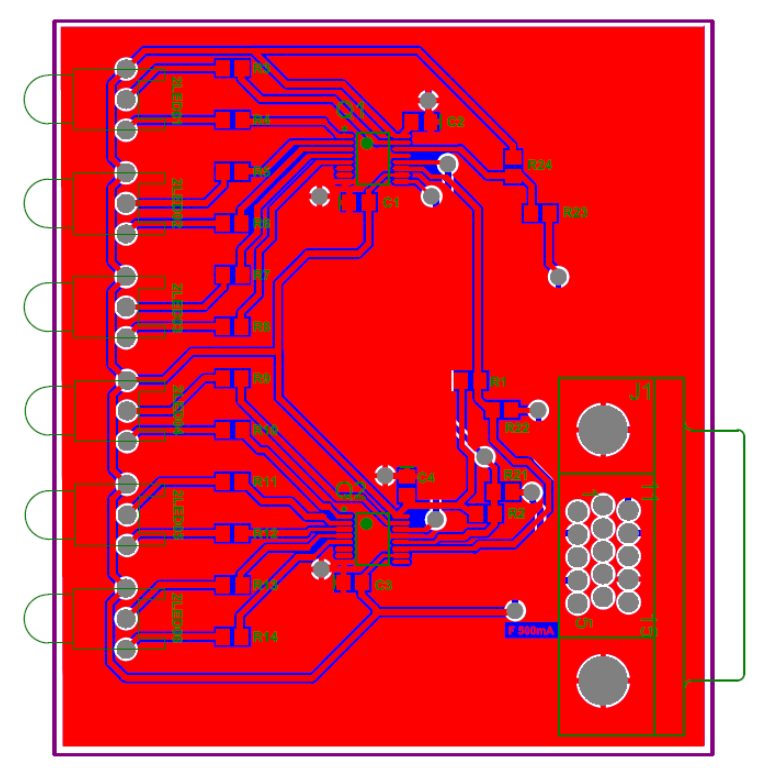

(a)

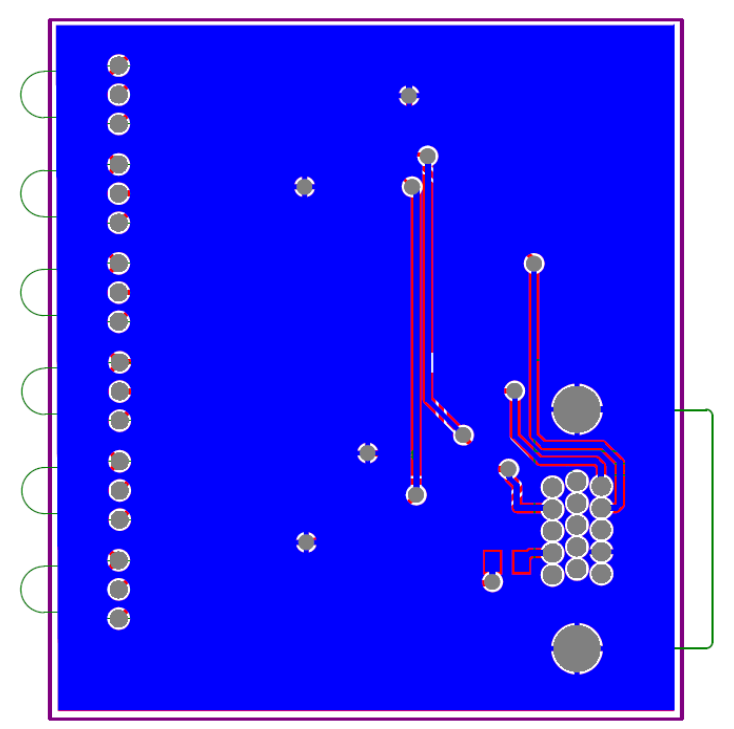

(b)

Figure A.1 PCB layout of CompactRIO MDK LED indicator module (a) top view and (b) bottom view 